\title{
La disputatio
}

\section{au cœur}

\section{du management}

Débats et controverses

sous la direction de

Aude Deville et al. 


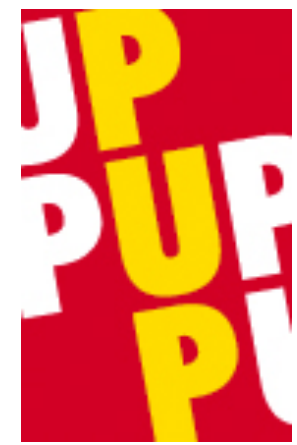

Presses universitaires de Provence

\section{La disputatio au cœur du management}

Débats et controverses

Aude Deville, Jérôme Dupuis, Jean-Fabrice Lebraty, Emmanuelle Nègre, Caroline Riché et Jean-François Sattin (dir.)

DOI : 10.4000/books.pup.56404

Éditeur: Presses universitaires de Provence

Lieu d'édition : Aix-en-Provence

Année d'édition : 2022

Date de mise en ligne : 8 février 2022

Collection : Travail \& Gouvernance

EAN électronique : 9791032003916

\section{Q OpenEdition}

\section{Books}

https://books.openedition.org

\section{Édition imprimée}

Date de publication : 15 février 2022

EAN (Édition imprimée) : 9791032003640

Nombre de pages : 270

\section{Référence électronique}

DEVILLE, Aude (dir.) ; et al. La disputatio au cœur du management : Débats et controverses. Nouvelle édition [en ligne]. Aix-en-Provence, France : Presses universitaires de Provence, 2022 (généré le 26 août 2022). Disponible sur Internet : <http://books.openedition.org/pup/56404>. ISBN :

9791032003916. DOI : https://doi.org/10.4000/books.pup.56404.

\section{Crédits de couverture}

Création graphique : Hokus Pokus, Rennes, France

(C) Presses universitaires de Provence, 2022

Conditions d'utilisation

http://www.openedition.org/6540 


\section{RÉSUMÉS}

Apparues dans le paysage académique au tournant des années 1970, après s'être émancipées des sciences juridiques et des sciences économiques, les sciences de gestion et du management occupent désormais une place centrale dans le dispositif universitaire français. En effet, un étudiant sur cinq y suit un cursus généraliste ou spécialisé en management des organisations, avec des taux d'insertion professionnelle atteignant des niveaux très élevés. Le dynamisme est au moins aussi significatif en matière de recherche, avec la présence d'associations académiques très impliquées dans la diffusion des savoirs théoriques et appliqués. Alors même que de multiples débats se font jour sur la gouvernance du système universitaire, notamment dans le processus de recrutement et de promotion des enseignants-chercheurs, il est essentiel de discuter sereinement des enjeux en présence. Tel est l'objet du présent ouvrage, dont les différents chapitres sont rédigés par 40 membres ou anciens membres de la section 06 du Conseil national des universités (CNU) de la mandature 2019-2023. Leur volonté est d'éclairer les controverses en optant pour une vision réflexive, fondée sur le registre de la disputatio: une discussion entre pairs mobilisant arguments et contre-arguments. Les 18 chapitres, organisés en cinq parties (mesurer, transmettre, s'interroger, explorer, se projeter), abordent des questionnements majeurs devant retenir l'attention de tous les enseignants-chercheurs en sciences de gestion et du management et, plus largement, du grand public qui souhaite mieux appréhender la gouvernance du système universitaire.

\section{AUDE DEVILLE (DIR.)}

Aude Deville est professeur des universités en sciences de gestion et du management à l'Université Côte d'Azur, en poste à l'IAE de Nice où elle dirige le Master Contrôle de gestion et audit organisationnel, après avoir créé et dirigé le Groupe de recherche en management (GRM), en assurant au niveau national la fonction de délégué scientifique AERES, puis HCERES. Ancienne co-rédactrice en chef de Comptabilité Contrôle Audit et de deux dossiers spéciaux de la Revue Française de Gestion, elle a publié de nombreux articles dans des revues nationales et internationales, notamment Management Accounting Research, Comptabilité Contrôle Audit, Revue Française de Gestion, Finance Contrôle Stratégie, Journal of Applied Accounting Research et Applied Economics, et a organisé la conférence de l'Association francophone de comptabilité en 2010. Elle est présidente de la section $06 \mathrm{du}$ CNU pour la mandature 2019-2023.

\section{JÉRÔME DUPUIS (DIR.)}

Jérôme Dupuis, après avoir été cadre dirigeant de collectivités locales et conseil en gestion des organisations publiques, est maître de conférences honoraire en sciences de gestion et du management à l'Université de Lille, où il a dirigé le Master Manager territorial. Ses travaux de recherche portent sur l'analyse comparée des systèmes locaux de performance au niveau européen et sur l'innovation des politiques et organisations publiques territoriales. Il est l'auteur d'une quinzaine d'articles dans des revues classées, de plus de 30 ouvrages, et de plus d'une centaine de contributions à des revues professionnelles. Il est membre du bureau de l'Association internationale de 
recherche en management public (AIRMAP) en charge des partenariats et des relations extérieures en France et en Europe.

\section{JEAN-FABRICE LEBRATY (DIR.)}

Jean-Fabrice Lebraty est professeur des universités en sciences de gestion et du management à l'Université Jean-Moulin Lyon III, où il est en poste à l'IAE de Lyon. Membre du laboratoire Magellan, il est spécialisé en management des systèmes d'information, avec des domaines de recherche concernant la prise de décision en situation extrême, le crowdsourcing et les technologies innovantes comme la blockchain. Sur ces thématiques, il est l'auteur de nombreux articles et de plusieurs ouvrages de référence. Il est membre élu du bureau de la section $06 \mathrm{du} C \mathrm{CNU}$ pour la mandature 2019-2023.

\section{EMMANUELLE NĖGRE (DIR.)}

Emmanuelle Nègre est maître de conférences en sciences de gestion et du management à l'Université Toulouse 1 Capitole, en poste à la Toulouse School of Management. Membre du laboratoire TSM Research, ses recherches s'inscrivent principalement dans le domaine de la comptabilité sociétale et de la comptabilité critique. Elles ont été publiées dans des revues nationales et internationales comme Comptabilité Contrôle Audit, Critical Perspectives on Accounting, Journal of Accounting \& Public Policy et M@n@gement. Elle est membre élue du bureau de la section 06 du CNU pour la mandature 2019-2023.

\section{CAROLINE RICHÉ (DIR.)}

Caroline Riché est maître de conférences en sciences de gestion et du management à l'Université Paris-Saclay, en poste à l'IUT de Sceaux où elle responsable de la Licence 3 Économie-Gestion, parcours Comptabilité contrôle. Membre du laboratoire Réseaux, Innovation, Territoires, Mondialisation (RITM), ses travaux de recherche s'inscrivent en comportement du consommateur et visent notamment à mieux comprendre les interactions sociales sous-jacentes à une décision collective et à une consommation partagée. Sur ces thématiques, elle est l'auteur de nombreuses publications dans des revues académiques et de vulgarisation et dans des conférences nationales et internationales. Elle est également rédactrice en chef de Management \& Data Science, revue scientifique de l'Association Information \& Management qui analyse l'impact de la transformation digitale sur le management (stratégie, marketing, ressources humaines, etc.). Elle est membre élue du bureau de la section $06 \mathrm{du} \mathrm{CNU}$ pour la mandature 2019-2023.

\section{JEAN-FRANÇOIS SATTIN (DIR.)}

Jean-François Sattin est maître de conférences HDR en sciences de gestion et du management à l'Université Paris 1 Panthéon-Sorbonne, où il codirige le Master 2 
Innovation, management des technologies et entrepreneuriat. Membre du laboratoire PRISM-Sorbonne et secrétaire général de l'Académie de l'entrepreneuriat et de l'innovation (AEI), ses travaux portent sur la finance entrepreneuriale ainsi que sur les conséquences des échecs entrepreneuriaux, appréhendées à la fois sous l'angle financier et sous l'angle psychologique. Sur ces thématiques, il est l'auteur de nombreuses contributions dans des revues académiques, dans des ouvrages collectifs, ainsi que dans des congrès nationaux et internationaux. Il est vice-président de la section $06 \mathrm{du} \mathrm{CNU}$ pour la mandature 2019-2023.

\section{NOTE DE L'ÉDITEUR}

Le présent ouvrage a bénéficié d'une aide à la publication des associations académiques et des institutions suivantes :

AEI (Académie de l'entrepreneuriat et de l'innovation);

affi (Association française de finance);

AFMAT (Association française de management du tourisme) ;

AGRH (Association francophone de gestion des ressources humaines);

AIRL-SCM (Association internationale de recherche logistique et Supply Chain Management) ;

AIRMAP (Association internationale de recherche en management public);

ARAMOS (Association de recherche appliquée au management des organisations de santé) ;

L'Observatoire ASAP (l'Observatoire de l'action sociétale et action publique);

IAE FRANCE, Écoles universitaires de management ;

IMPGT (Institut de management public et gouvernance territoriale), Aix-Marseille Université ;

FNEGE (Fondation nationale pour l'enseignement de la gestion des entreprises).

Qu'elles en soient chaleureusement remerciées. 


\section{La disputatio \\ au cœur \\ du management}

Débats et controverses

sous la direction de

Aude Deville et al. 



\section{Travail \\ Gouvernance}

collection dirigée par Ghislaine Gallenga \& Gilles Paché

Dans la même collection:

Sylvie Avignon, dir., Droit, management et société. Débats contemporains, 222 p., 2022

Gérard Cliquet (avec la collaboration de Rozenn Perrigot), Franchise et autres réseaux. Cadres théoriques et enjeux managériaux, 270 p., 2021

Camal Gallouj, Faïz Gallouj et Nizar Gallouj, dir., Commerce et distribution dans les pays du Maghreb. Acteurs, enjeux et stratégies, 342 p., 2021

Camal Gallouj et Gilles Paché, dir., Préface Stephen Vargo, Management des services. Convergences, contrastes et controverses, 354 p., 2020

Rémi Mencarelli et Arnaud Rivière, dir., La valeur perçue en marketing. Perspectives théoriques et enjeux managériaux, 287 p., 2020

Jean-Pierre Bréchet, L'action collective. Une perspective régulationniste, 140 p., 2019

Nathalie Fabbe-Costes et Aurélien Rouquet, dir., La logistisation du monde. Chroniques sur une révolution en cours, 334 p., 2019

Aurélie Condevaux, Performances polynésiennes. Adaptations locales d'une «formule culturelle-touristique » globale en Nouvelle-Zélande et à Tonga, 358 p., 2018

Melissa Blanchard, Travail, sexualité et migration. Les commerçantes sénégalaises à Marseille, 152 p., 2018

Jean Nollet, Stratégies d'achat. Enjeux et perspectives, 224 p., 2018

Rozenn Perrigot, dir., Retailing et localisation. Une approche multidisciplinaire, 354 p., 2018

Lise Gastaldi, Cathy Krohmer et Claude Paraponaris, dir. Activités et collectifs. Approches organisationnelles et cognitives, 244 p., 2017

Michel Marchesnay, L'entrepreneuriat. Des logiques aux pratiques d'action, 200 p., 2017

Lise Gastaldi, Cathy Krohmer et Claude Paraponaris, dir., Activités et collectifs. Approches cognitives et organisationnelles, 236 p., 2017

Michel Pendaries, Piloter la performance organisationnelle. Une aide à la décision avec la valeur ajoutée horaire, 128 p., 2017

Annie Lamanthe et Stéphanie Moullet, dir., Vers de nouvelles figures du salariat. Entre trajectoires individuelles et contextes sociétaux, 184 p., 2016

Gilles Paché et Mustapha El Khayat, dir., Invitation aux flux. Entre transport et espace, 250 p., 2016 


\title{
La disputatio \\ au cour du management
}

Débats et controverses

\author{
sous la direction de \\ Aude Deville, Jérôme Dupuis, \\ Jean-Fabrice Lebraty, Emmanuelle Nègre, \\ Caroline Riché et Jean-François Sattin
}


Le présent ouvrage a bénéficié d'une aide à la publication des associations académiques et des institutions suivantes.

Qu'elles en soient chaleureusement remerciées.
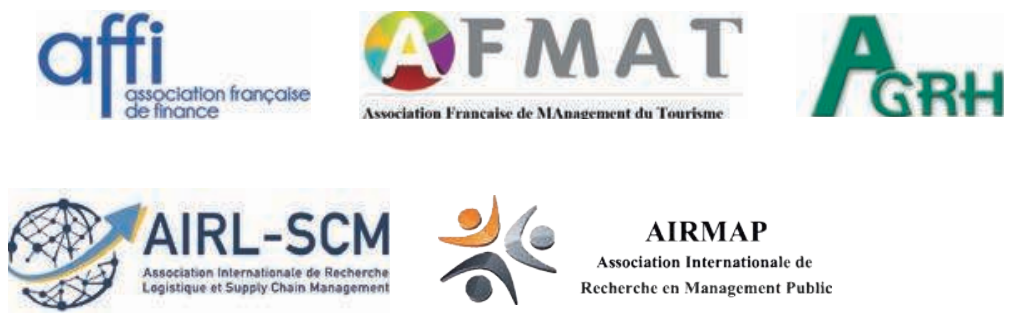

\section{AIRMAP}

Association Internationale de Recherche en Management Public

\section{ARAMOS}

OBSERVATOIRE

๑ Presses Universitaires de Provence

Aix Marseille Université

29, avenue Robert-Schuman - F - 13621 Aix-en-Provence CEDEX 1

Tél. 33 (0)4 13553191

pup@univ-amu.fr - Catalogue complet sur presses-universitaires.univ-amu.fr/editeur/pup

facebook.com 


\title{
Introduction générale
}

\author{
Aude Deville, Jérôme Dupuis, Jean-Fabrice Lebraty, Emmanuelle NèGre, \\ Caroline Rıché et Jean-François SATtıN
}

Le présent ouvrage incarne une volonté : réunir des membres élus et nommés, titulaires et suppléants, de la section 06 du CNU 06 autour d'un travail fondé sur la collaboration et la réflexion, au-delà du rythme des sessions (qualification, promotion et PEDR), et au service des sciences de gestion et du management. L'idée de produire tel ouvrage collectif a émergé lors de la première session de la mandature 2019-2023, à savoir la session de qualification aux fonctions de maître de conférences et de professeur des universités (voie 46.1) qui s'est tenue en janvier 2020. L'environnement universitaire est en forte mutation et évolue actuellement de façon inquiétante. La Loi de programmation de la recherche (LPR), notamment, a été votée le 24 décembre 2020, malgré l'opposition d'une grande partie du monde universitaire, et au moment où s'achève la rédaction de l'ouvrage, la communauté a vu les premiers décrets d'application publiés le 20 et 29 décembre 2021. À l'heure où nous écrivons ces lignes, les missions du CNU restent toutefois encore diversifiées et indispensables à la cohésion de la communauté académique.

\section{Des décisions du CNU s'inscrivant dans de grands principes}

Les décisions de la section 06 du CNU s'exercent selon de grands principes qui sont rappelés dans les rapports de sessions, et qu'il sera possible de retrouver de manière développée tout au long de l'ouvrage. À ce titre, ils s'inscrivent à la fois dans la durée et dans la continuité des pratiques initiées par les précédentes mandatures. Ces grands principes peuvent être résumés ainsi :

- L'intégrité. Les décisions et arbitrages sont réalisés sous le prisme de l'intégrité de la discipline. Les sciences de gestion et du management ont un périmètre à définir et à rappeler à chaque instant afin de préserver l'intégrité disciplinaire.

- L'équité de l'évaluation des dossiers. Le processus d'allocation des rapporteurs est collégial. Pour les promotions et les PEDR, les rapporteurs ne se connaissent pas. La finalité est de permettre à chaque dossier de faire l'objet d'une évaluation fidèle, produite de manière indépendante par les rapporteurs. Les décisions, 
notamment pour les promotions et les PEDR, sont contraintes par un nombre contingenté d'avis favorables. Ne pas obtenir une promotion au niveau national ou une note de $A$ à la PEDR ne signifie pas nécessairement que le dossier est mal valorisé, mais résulte souvent d'une comparaison relative à d'autres dossiers très bons. En sciences de gestion et du management, les dossiers déposés sont souvent de grande qualité, conséquence notamment d'une autocensure de certains collègues. Nous rappelons que les rapporteurs du CNU évaluent des dossiers, et non des candidats.

- L'homogénéité et l'indépendance. II est essentiel de préserver une évaluation homogène des dossiers. L'homogénéité ne peut être garantie que par l'indépendance des acteurs et par un processus de décision transparent. Ainsi, par exemple, la qualification aux fonctions de professeur des universités par la voie du 46.1, lorsqu'elle existait, contribuait à légitimer nationalement et disciplinairement des dossiers qualifiés sur la base d'une évaluation indépendante et homogène de l'ensemble des candidatures soumises.

- La collégialité des décisions. Concernant la qualification aux fonctions de maître de conférences, 48 collègues ( 24 collègues du collège $A$, et 24 collègues du collège $B$ ) contribuent à la décision de qualification ou de non-qualification des dossiers. Ces décisions sont fondées sur l'usage d'outils élaborés par des pairs, tels que le classement FNEGE, par exemple. Néanmoins, le cénacle section 06 du CNU n'est pas une chambre d'enregistrement. Les décisions dépendent surtout de la connaissance des communautés académiques, connaissance portée par les membres du CNU au travers d'échanges et débats qui ont lieu lors des sessions. Cette connaissance est déterminante lors des discussions et de la prise de décision collégiale autour des dossiers évalués.

\section{Structure de l'ouvrage}

L'ouvrage proprement dit se décompose en cinq parties (" mesurer », " transmettre », " s'interroger ", " explorer ", " se projeter ") et 18 chapitres. Nous remercions les collègues pour leurs contributions et leur investissement au service des sciences de gestion et du management. Nous remercions également les soutiens reçus de la part des associations savantes et des institutions actrices de notre monde académique. Une analyse lexicale des différents chapitres, illustrée par le nuage de mots de la figure 1 , signale les préoccupations majeures des collègues ayant contribué. Ainsi, les trois missions dévolues aux enseignants-chercheurs sont au cœur de l'ouvrage : l'activité scientifique, l'activité pédagogique et l'engagement auprès de nos communautés (institutions et sociétés savantes). Les pratiques, les étudiants, les missions, le don, le travail, les compétences, la carrière, l'engagement, les critères d'évaluation, les entreprises, les terrains de recherche et, bien sûr, nos universités constituent les principaux thèmes développés dans les différents chapitres que nous invitons à découvrir. 


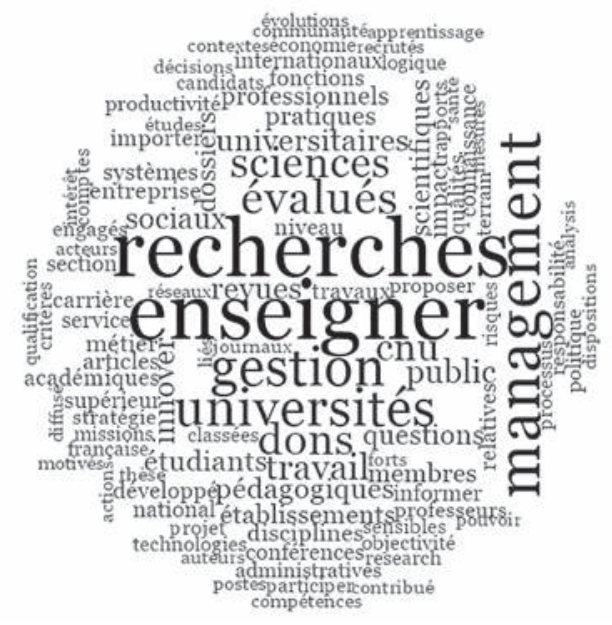

Figure 1 : Mots clés issus de l'analyse des contributions à l'ouvrage

\section{Mesurer}

La première partie discute l'usage de l'évaluation quantitative ainsi que la place du chiffre dans l'appréciation du contenu des dossiers soumis au CNU. Elle interroge de manière plus générale sur l'évaluation constante à laquelle est soumis tout enseignant-chercheur au cours de sa carrière : une évaluation de sa pédagogie par les étudiants, une évaluation de la recherche au niveau national et international, et une estimation de l'engagement institutionnel et scientifique local et national. La question du don à la communauté se pose car si l'évaluation est constante, permanente, voire omniprésente, la valorisation des contributions n'est pas toujours au rendez-vous. Le bon fonctionnement de la communauté académique et universitaire repose en effet sur l'engagement des collègues, souvent naturel et spontané (conseils aux collègues, supervision des travaux des étudiants, discussion d'articles, évaluation d'articles, participation aux jurys de thèses et d'HDR, encadrement de mémoires et de thèses, recrutement d'étudiants, participation aux comités de sélection, engagement dans des instances telles que le CNU ou l'HCERES, etc.).

\section{Transmettre}

La transmission de la connaissance est au cœur du métier d'enseignant-chercheur. Elle prend un sens particulier en sciences de gestion et du management car, si l'apport des connaissances théoriques et méthodologiques est essentiel, l'insertion des étudiants dans des organisations publiques ou privées et la considération de l'environnement socio-économique présentent également des enjeux majeurs. À cet égard, les pédagogies innovantes et les formations en apprentissage suscitent l'intérêt et offrent de nouvelles perspectives. Leur déploiement nécessite de nombreuses ressources : financières, de temps et de développement de nouvelles compétences, etc. C'est l'objet de la deuxième partie de l'ouvrage. 


\section{S'interroger}

L'enseignant-chercheur porte l'interrogation en lui, c'est un moteur d'innovation, d'apprentissage permanent et de progression. Dans la troisième partie sont questionnées la culture organisationnelle de l'enseignant-chercheur (équité, justice, mais aussi stress au travail), ainsi que l'attractivité du métier pour les jeunes collègues (docteurs et doctorants, mais aussi futurs étudiants qui envisagent un parcours recherche). Les nouveaux enjeux de la recherche sont discutés, notamment la prise de conscience croissante du rôle et des résultats de la recherche qui doivent être portés au-delà des frontières académiques. Leur exportation et l'évaluation de leurs impacts socio-économiques deviennent un enjeu majeur, avec une interrogation sur une revalorisation de la recherche en sciences de gestion et du management. Les grands principes du métier sont rappelés en plaçant l'indépendance au cœur de nos organisations et du quotidien de chercheur.

\section{Explorer}

La recherche en sciences de gestion et du management porte sur les organisations publiques et privées, et s'étend aux différentes fonctions de l'entreprise (comptabilité et contrôle, finance, marketing, gestion des ressources humaines, gestion de production et logistique, gestion des systèmes d'information et de communication, gestion juridique et fiscale, management stratégique), mais aussi aux pratiques managériales et au rôle du manager. Elle met en œuvre des méthodologies d'analyse qualitatives ou quantitatives en fonction de la question de recherche traitée. La quatrième partie passe en revue des cas d'analyses bien connus, et d'autres moins, réalisés en sciences de gestion et du management. Incontestablement un monde à redécouvrir et découvrir.

\section{Se projeter}

Le vote de la LPR fin 2020 rend délicat l'exercice de projection. Néanmoins, des collègues s'y sont attachés. Les membres du CNU sont élus ou nommés pour quatre ans, avec la possibilité d'un renouvellement de leur mandat. II s'agit de collègues enseignants-chercheurs, directeurs d'étude, directeurs de laboratoire, membres actifs au sein des bureaux des sociétés savantes, rapporteurs pour des revues scientifiques, rédacteurs de revues, membres de jury de thèses et d'HDR et de comités de sélection, ou encore membres dans les instances locales. Les membres du CNU partagent le quotidien de chaque collègue et pratiquent l'enseignement et la recherche au sein de la discipline. In fine, le CNU est une assemblée de pairs qui étudient les demandes formulées par les collègues selon leur avancée dans la carrière. Dans la cinquième et dernière partie de l'ouvrage, les enjeux à ce niveau sont discutés, au regard de l'environnement changeant que nous avons connu en 2020-2021, à la fois avec justesse, souci du lendemain, mais aussi bienveillance et optimisme. 
Mesurer 



\title{
Chiffres et décision
}

\author{
La part des pairs
}

Aude DevilLe et Emmanuelle NèGre

Le milieu universitaire français a connu et connait actuellement plusieurs réformes (par exemple, la loi d'autonomie des universités ou la loi de programmation de la recherche). Elles tendent à faire évoluer non seulement le statut des enseignants-chercheurs et leurs perspectives de carrière, mais aussi le contenu même de leurs activités et leurs conditions de travail. L'évaluation des enseignants-chercheurs apparaît au cœur des évolutions connues par la profession. Dans ce cadre, l'objectif du chapitre est de questionner d'abord le pouvoir performatif des chiffres et des évaluations quantitatives (le chiffre en tant que créateur de réalité) et, ensuite, le contrôle que peut exercer le chiffre sur l'évalué. Enfin, nous discutons l'évaluation des enseignants-chercheurs par la section 06 du CNU en prenant l'exemple de la qualification aux fonctions de maître de conférences. Jusqu'à aujourd'hui (2022), la qualification aux fonctions de maître de conférences fait partie des missions du CNU et fait l'objet d'une session spécifique selon un calendrier précis énoncé par le ministère de l'enseignement supérieur, de la recherche et de l'innovation.

Chaque section du CNU organise sa session et qualifie - ou non - les dossiers en fonctions de critères propres à la discipline. Pour la section 06, les critères portent sur l'ensemble des volets qui font la richesse et la complexité du métier d'enseignant-chercheur en sciences de gestion et du management : la production scientifique (articles publiés, communications en conférences, ouvrages et chapitres d'ouvrages, rapports d'activités, cas pédagogiques, articles à rayonnement socio-économique, etc.), l'activité pédagogique et l'engagement collectif national et local, institutionnel et scientifique $^{1}$. Les critères qui supportent la décision de qualifier ou de ne pas qualifier vont au-delà de l'analyse et la relecture de la thèse et des rapports de soutenance, même si ces éléments sont lus avec attention. Les pairs, réunis lors de la session de qualification (24 maîtres de conférences et 24 professeurs des universités), discutent

1 Les rapports de session sont déposés sur le site du conseil national des universités : [https://www. conseil-national-des-universites.fr/cnu/\#/entite/entiteName/CNU/idChild/31/idNode/3696-3723], consulté le 6 juillet 2021. 
chaque dossier et développent des arguments favorables ou défavorables à partir d'éléments chiffrés, mais pas seulement.

\section{La construction d'une réalité performative par le chiffre}

Muniesa et Callon (2013) rappellent que la notion de " performativité » est empruntée à la pragmatique du langage. Austin (1991) énonce le premier que le langage peut se distinguer en deux types d'énoncés : les énoncés constatatifs et les énoncés performatifs. Les énoncés constatifs sont vrais ou faux. À la différence, les énoncés performatifs ne sont ni vrais ni faux, mais ils sont susceptibles de réaliser un changement (Dumez, 2015). En d'autres termes, la performativité renvoie à " la capacité de certains énoncés délibérés à changer la réalité et les pratiques qu'ils désignent » (Aggeri, 2017). Ainsi, la réalité n'est pas seulement décrite mais aussi modelée par ces énoncés (MacKenzie et Millo, 2003), qui peuvent pr endre différentes formes (actes de langage, actes de calcul, etc.). Lorsque les énoncés se réalisent, Austin (1991) les qualifient de felicitous et lorsque ce n'est pas le cas de infelicitous.

Le concept de performativité a fait l'objet de nombreuses utilisations au sein des sciences sociales (MacKenzie et al., 2007). Plus particulièrement, l'effet performatif du " chiffre " a été étudié selon deux principales approches. L'une concerne les « actes de calculs » définis par Fauré et Gramaccia (2006) comme des « actes de langage énonçant un calcul ou un chiffre ". Au sein de ce courant, les études s'intéressent à la manière dont les chiffres mobilisés par différents acteurs dans des calculs énoncés, oralement ou par écrit, construisent une réalité (Fauré et al., 2010). L'autre porte sur les chiffres et les calculs en tant que tels et regroupe, par exemple, les travaux en lien avec la performativité de la comptabilité au sens large (Vollmer, 2007 ; Revellino et Mouritsen, 2015). L'idée d'une comptabilité qui participe à la construction du Monde, et qui n'est pas seulement le reflet d'une réalité, n'est pas nouvelle et a déjà été soulignée par plusieurs auteurs (Hines, 1988).

La production et la communication de chiffres relèvent de ce qu'Espeland et Stevens (2008) nomment la quantification. Plusieurs travaux ont mis en évidence que la quantification peut inciter les individus à adopter certains comportements (Espeland et Sauder, 2007). En effet, la quantification ne se contente pas de fournir un reflet du monde; elle crée une nouvelle façon de le penser, de le représenter, de l'exprimer et d'agir sur lui (Desrosières, 1993). En d'autres termes, la quantification est dotée d'une capacité à agir sur le réel, d'où l'expression de " performativité de la quantification ». Espeland et Sauder (2007) développent, en outre, le concept de réactivité selon lequel les individus modifient leur comportement en réaction à une évaluation, une observation ou une mesure. Pour Martin (2020), la performativité de la quantification est d'autant plus grande que les chiffres deviennent non seulement un objectif à atteindre pour des acteurs, mais aussi un instrument d'évaluation de ces acteurs.

Appliquée au milieu universitaire, il apparaît que l'évaluation quantifiée de l'activité de recherche des enseignants-chercheurs avait initialement comme objectif de "vérifier le respect du contrat de travail universitaire ", autrement dit, de mesurer le travail de recherche des enseignants-chercheurs (Guillon, 2016). Très vite, l'objectif s'est déplacé vers une incitation des universitaires à publier dans des revues classées. 
Pour Boulet et Harari-Kermadec (2014), une telle évaluation et son pendant qu'est la production de classements " ne servent pas tant à produire une information sur les activités qu'à fournir des incitations à se comporter d'une certaine manière, par exemple à produire de la recherche économiquement utile ». L'adaptation des enseignants-chercheurs à l'évaluation et à la mesure de leur " productivité " a eu des effets considérables sur l'évolution des pratiques de publications aussi bien en termes de quantité et de fréquence qu'au niveau des supports de publications utilisés. Power (1999) résume la situation de la manière suivante :

Les cycles de recherche ont évolué en faveur de publications dans des revues prestigieuses plutôt que dans des livres. Les scientifiques changent leurs habitudes de recherche et tout un ensemble d'activités pour lesquelles des mesures de performance n'ont pas été conçues ont cessé d'avoir une valeur officielle. L'édition de livres, l'organisation de conférences et, paradoxalement, l'examen et la facilitation des efforts de publication des autres ne sont plus pris en compte.

Le dialogue avec le monde de la pratique a également été découragé par ces nouvelles mesures, une telle activité ne permettant pas d'aboutir à des résultats qui comptent dans l'évaluation des universitaires (Hopwood, 2008). Gebreiter (2022) discute l'évolution de l'évaluation des activités de recherche et d'enseignement qui peut conduire à la domination d'une orientation marché pour laquelle les activités de recherche et d'enseignement sont estimées à partir d'outputs produits et les étudiants endossent le rôle de clients. Il analyse en particulier le cas des départements de management en Angleterre. Son analyse le mène à indiquer que cette vision tournée systématiquement vers l'output conduit à séparer les activités de recherche et d'enseignement. Certains collègues deviennent ainsi des spécialistes de la recherche et d'autres de l'enseignement.

Il devient dès lors plus difficile de faire valoir des activités et pratiques de recherche au sein des enseignements car les collègues identifiés "spécialistes " de recherche n'enseignent que peu (ou plus du tout). Une hiérarchie apparait au sein du corps professoral : les profils " recherche " sont mieux « valorisés » que les profils " enseignement ", créant des tensions au sein des institutions. Ce qui peut apparaître plus dangereux, c'est également l'émergence d'une hiérarchie entre les disciplines de management. En effet, il a été identifié par les dirigeants d'écoles de management (publics ou privées) des disciplines où il est plus aisé de publier dans des revues bien valorisées dans les classements internationaux, et d'autres non. Dans une perspective de moyen / long terme, certaines disciplines sont perçues comme intensive en pédagogie (Gebreiter [2022] cite la comptabilité), et d'autres disciplines comme intensive en recherche. Ainsi, le triste sort des sciences de gestion et du management peut être réduit à une typologie disciplinaire. Les chiffres sont utilisés pour promouvoir un tel scénario et le rôle des pairs dans le processus de décision s'en trouve diminué.

Plus généralement, nombreuses sont les études qui ont souligné les dangers de la seule évaluation quantitative des enseignants-chercheurs. Hopwood (2008) craint une standardisation des recherches puisque la plupart des revues les plus « prestigieuses " tendent à publier désormais un seul type de recherche : de la recherche mainstream telle qu'elle est effectuée en Amérique du Nord. Pour l'auteur, le risque est désormais que l'acte de publication compte plus que le contenu lui-même de la publication. Autrement dit, il s'agirait plus aujourd'hui de publier des papiers, 
même s'ils n'apportent rien à la connaissance, que de véritablement faire de la recherche. Plusieurs questionnements se posent donc concernant, par exemple, la capacité des classements à participer de manière pertinente à l'évaluation de la création de savoirs.

En outre, il arrive que le réel soit modifié dans le sens inverse de celui prévu au départ, c'est ce qui est qualifié dans la littérature d'effets contre-performatifs (Callon, 2010). Dans une telle configuration, les individus adoptent des comportements qui sont en désalignement avec les buts recherchés. Broedker et al. (2020) indiquent, par exemple, comment la mise en place d'un système de classement du capital intellectuel au sein d'une organisation publique australienne a eu des effets contre-performatifs dans le sens où elle a généré des résistances qui ont affaibli la stratégie de l'entreprise en la matière. Pour Letiche et al. (2017), les enseignants-chercheurs craintifs, effrayés, peu sûrs d'eux-mêmes, ne peuvent pas innover et/ou être créatifs, ce qui ne peut in fine aboutir qu'à l'effet inverse de celui recherché par l'introduction de l'évaluation. Néanmoins, le chiffre reste un élément incontournable du processus d'évaluation. La gestion et l'évaluation de " masse " induit la présence de chiffres et de pratiques de quantification de l'activité des évalués. Nous ne le contestons pas. Néanmoins se pose la question de son usage et de son importance au sein du processus d'évaluation.

\section{Le chiffre : un élément de l'évaluation}

Pour Mårtensson (2009), la mesure peut être considérée comme un « processus par lequel plusieurs dimensions sont d'abord simplifiées et réduites en nombre afin de pouvoir être mesurées ". Le désir ou besoin humain de quantifier le monde par diverses mesures n'est pas nouveau et semble être sans limite. Dermakar et Gendron (2019) évoquent à ce sujet une socialisation à grande échelle mettant en exergue la pertinence, voire la beauté de ce qui peut être chiffré avec précision (Rose, 1991) et, au contraire, l'inconfort et la méfiance à l'égard de ce qui ne peut être quantifié (Porter, 1995). Il est intéressant de comprendre les raisons de la généralisation des pratiques de chiffrage ou quantification. La littérature critique en comptabilité offre une grille de lecture intéressante pour éclairer ce point.

Cette dernière souligne en effet la manière dont les dispositifs et outils comptables, loin d'être neutres, peuvent être utilisés pour surveiller et discipliner les membres d'une organisation. De manière similaire, Miller et O'Leary (1987) présentent la comptabilité - en particulier les coûts standards - comme un dispositif visant à rendre les individus gouvernables. En outre, selon plusieurs auteurs, dont Farjaudon et Morales (2013), le système comptable, ainsi que l'usage qui en est fait, constitue un outil de domination renforçant l'hégémonie des dirigeants sur les autres parties prenantes. Sous une artillerie mathématique produisant de l'illusion, les chiffres, loin d'être objectifs et neutres, répondent en réalité aux objectifs de ceux qui détiennent le pouvoir. En effet, pour Mårtensson (2009), les détenteurs du pouvoir utilisent la mesure quantitative " comme un outil pour développer une société rationnelle et efficace ". Certes, les chiffres rassurent et aident à la décision, mais ils ne peuvent pas faire la décision. Dans certaines situations, le recours systématique aux chiffres peut même apparaître comme confortable car cela permet de 
déresponsabiliser les preneurs de décisions. Néanmoins, dans le cas d'activités où les pairs jouent un rôle prépondérant dans le processus de reconnaissance et de légitimation des compétences et de l'expérience, un trop grand pouvoir décisionnel donné aux chiffres calculés de façon ad hoc peut s'avérer périlleux.

Par l'énoncé des chiffres, par le comptage, certaines choses deviennent visibles et pas d'autres; ce qui est compté compte et doit être compté, ce qui leur confère du pouvoir (Fauré et Gramaccia, 2006). Sur cette base, une question intéressante demeure et pourrait être soulevée. Elle « concerne non seulement ce à quoi les mesures contribuent, mais aussi ce qu'elles négligent en le faisant. Comme le dit l'adage, ce qui est mesuré est géré, donc ce qui n'est pas mesuré n'est pas géré » (Mårtensson, 2009). De quoi cherche-t-on à nous détourner en focalisant notre attention sur les chiffres ? L'étude des différents pans de littérature nous enseigne donc qu'en cherchant à réduire les enseignants-chercheurs à la seule chose qui est visible, c'est-à-dire ce qui est quantifiable, il y a l'idée de vouloir mieux les contrôler, et par conséquent mieux les soumettre.

Pour Galvez-Behard (2010), " l'impossibilité de discuter les critères et le contenu de l'évaluation peut engendrer un sentiment de violence symbolique dont peuvent découler un grand nombre d'effets pervers (nouvel arbitrage entre l'enseignement et la recherche, désinvestissement dans les tâches d'intérêt collectif difficilement reconnues, etc.) ". La conséquence d'objectifs chiffrés à réaliser est que le travail de l'enseignant-chercheur n'est plus appréhendé dans sa globalité, et surtout dans sa réalité. Les universitaires sont désormais traités comme des éléments interchangeables et doivent rendre compte de leur " performance ". C'est donc de plus en plus un langage issu du monde de l'entreprise que l'on retrouve au sein des universités et une vision managériale qui s'impose aux enseignants-chercheurs par le biais de l'évaluation quantifiée. II en découle une sorte de marchandisation de la connaissance (Insel, 2009), voire de l'université toute entière (Boulet et Harari-Kermadec, 2014), qui entre en totale déconnexion avec la liberté d'initiative, de recherche et d'esprit qui caractérise normalement la profession (Zarka, 2009).

Le chiffre et le processus de quantification est une technique sociale qui trouve sa légitimité dans un idéal : les mathématiques et la géométrie (Porter, 1995). Il existe des débats dans la littérature en comptabilité qui sont rappelés par Porter (1995) :

Considérée comme un phénomène social, la comptabilité est bien plus puissante et problématique que le réalisent les chercheurs et les journalistes.

Les débats ont également fait l'objet de discussions dans la revue Comptabilité Contrôle Audit au moment du choix à la faveur des IFRS (Burlaud et Colasse, 2010 ; Raffournier, 2011). La comptabilité est un bon exemple du chiffre porteur de l'intention de celui qui le construit. Il peut être néanmoins fiable et fidèle, une estimation au plus proche d'une réalité. L'usager, l'évaluateur, l'évalué doivent avoir conscience de l'implication de la règle de calcul mise en œuvre pour produire le chiffre. L'usage du chiffre par le décideur devrait se faire en conséquence. L'évaluation par le chiffre ou l'évaluation quantifiée peuvent être considérées comme un mal nécessaire ou un bienfait. Néanmoins, le chiffre ne peut être un garant à lui seul d'objectivité, de transparence ou d'intégrité (Porter, 1995). Pour cela il y a besoin d'experts concernés et engagés porteurs de buts et de projets. 


\section{Chiffre et évaluation par les pairs : le cas de la qualification des maîtres de conférences}

Conscients des dangers du seul usage du chiffre pour l'évaluation des enseignants-chercheurs, la section 06 du CNU a à cœur de conserver une démarche où le qualitatif joue un rôle important. Afin d'illustrer le propos, nous prendrons l'exemple de l'examen des dossiers de candidatures dans le cadre d'une demande de qualification aux fonctions de maître de conférences. Deux phases sont ici distinguées : avant et pendant la séance.

\section{Avant la séance}

La volonté de la section 06 du CNU d'inscrire sa démarche avant tout dans une approche qualitative, et pas seulement quantitative, peut être tout particulièrement soulignée au regard des documents demandés dans la constitution du dossier en lien avec une demande de qualification. En effet, outre les documents obligatoires exigés par le ministère de l'enseignement supérieur, de la recherche et de l'innovation (rapport de soutenance, CV, pièce justificative de la possession du doctorat, etc.), la section demande un certain nombre de documents, qui apportent des compléments d'information bien souvent utiles à l'examen complet des dossiers (thèse, pré-rapports autorisant la soutenance de la thèse, lettre de " motivation », etc.).

L'examen des dossiers par les rapporteurs s'effectue à l'aide d'une fiche de travail qui incite l'évaluateur à mettre la dimension qualitative au cœur de son analyse. La fiche demande au rapporteur, par exemple, non seulement de recenser les principales caractéristiques de la thèse (titre, jury, discipline), mais aussi d'en synthétiser les principaux apports et limites. Afin d'effectuer une telle synthèse, le rapporteur est amené à consulter les pré-rapports autorisant la soutenance de la thèse et le rapport de soutenance proprement dit, mais aussi à examiner la thèse afin de se faire sa propre opinion de celle-ci. À ce titre, le rapport sur la qualification aux fonctions de maître de conférences (session 2020) de la section 06 du CNU souligne :

Bien que les deux rapporteurs disposent du rapport de soutenance, et des pré-rapports, ils évaluent la thèse de façon indépendante et ne sont pas liés par l'appréciation du jury de soutenance. La thèse présente ainsi l'avantage d'avoir fait l'objet d'une double évaluation : par le jury de soutenance et par les rapporteurs du $\mathrm{CNU}^{2}$.

S'agissant des activités de recherche du candidat, la diversité des supports de publications est explicitement reconnue puisque la fiche de travail invite l'évaluateur à répertorier non seulement les publications dans des revues (classées ou non classées), mais aussi les communications lors de conférences, les ouvrages (labellisés ou non labellisés par la FNEGE) et les chapitres d'ouvrages (labellisés ou non labellisés par la FNEGE). Il est ensuite demandé à l'évaluateur d'effectuer un commentaire des productions scientifiques envoyées par le candidat, ce qui nécessite là encore que le rapporteur prenne pleinement connaissance des documents fournis.

2 Disponible sur le site du Conseil national des universités : [https://www.conseil-national-des-universites.fr/data/document/3731/2646/Public/Rapport\%20d\%20activit\%C3\%A9s/Rapport\%20 session\%20CNU\%2006\%20pour\%20la\%20qualification\%20MCF_MARS2020.pdf], consulté le 16 avril 2021. 
Bien sûr, les autres volets du métier d'enseignant-chercheur - les activités d'enseignement et administratives - sont également étudiés. S'agissant de l'enseignement, une pluralité de critères qualitatifs sont valorisés par les rapporteurs tels que la diversité des enseignements réalisés ou encore la diversité des publics auprès desquels les enseignements sont effectués. Au niveau administratif, chaque implication collective du candidat, quelle que soit sa forme, est valorisée par le rapporteur. C'est sur la base de cette fiche de travail que les rapporteurs rédigent leur avis motivé de synthèse, contenant des éléments factuels, qui sera ensuite repris dans le rapport officiel. Les rapporteurs sont enfin amenés à choisir une note $(A, B$ ou $C)$ pour chaque dossier étudié.

\section{Durant la séance}

L'examen d'un dossier débute par une mention par les deux rapporteurs de leur note respective pour le dossier discuté. La note n'est jamais donnée seule; elle s'accompagne toujours d'un argumentaire plus ou moins court de la part de chacun des rapporteurs. Les membres du CNU peuvent à tout moment intervenir pour demander des compléments d'information aux rapporteurs. Le rapport sur la qualification aux fonctions de maître de conférences (session 2020) de la section 06 du CNU 06 précise :

Si les membres du CNU s'estiment suffisamment informés sur le candidat, deux $A$ ou une évaluation $A / B$ conduisent à une qualification. De la même façon, deux $C$ conduisent à une non-qualification. Une évaluation $B / B$ donne lieu à une présentation plus détaillée du dossier. Dans les cas les plus limites (B/C ou $A / C)$, une discussion est ensuite ouverte. La décision de qualification est toujours prise par l'ensemble des membres.

On voit bien ici que l'échange va bien au-delà d'une simple confrontation de notes pour laisser place à de véritables discussions entre les rapporteurs mais aussi et surtout avec l'ensemble des membres de la section, particulièrement lorsque les rapporteurs sont en désaccord entre eux. Pour reprendre les mots de Zarka (2009), il s'agit par ce biais de corriger la subjectivité inhérente à l'étude de chaque dossier par la confrontation des subjectivités, et non par une prétention illusoire à l'objectivité absolue (quantitative).

Un autre exemple de la place occupée par le qualitatif dans l'évaluation des dossiers concerne la combinaison des trois critères que sont la recherche, l'enseignement et l'administratif. L'évaluation se fait selon une approche qualitative dans le sens où il n'y a pas de système de notes avec un calcul de moyennes, mais bien une évaluation qualitative laissant la place à une possibilité de compensation entre les critères. Le rapport sur la qualification aux fonctions de maître de conférences (session 2020) de la section 06 du CNU précise à ce sujet :

L'impression laissée par un dossier recherche de qualité moyenne peut être corrigée par une activité pédagogique dense et originale ou des engagements importants, par exemple au sein du laboratoire de recherche (...). Par ailleurs, les critères de l'impact sociétal et de l'engagement collectif vis-à-vis de la communauté académique sont de réel plus à promouvoir. 
Si la section 06 du CNU souhaite ardemment conserver un fonctionnement collégial où la discussion, l'échange et l'examen qualitatif des dossiers sont privilégiés, il ne peut s'abstenir de toute évaluation quantitative des dossiers reçus. Ainsi, nous l'avons indiqué, chaque dossier fait l'objet d'une double notation par les rapporteurs, et cette note sert de base à l'échange qu'il va y avoir ensuite avec les membres de la section. De même, les rapporteurs utilisent au moins en partie des classements, et notamment celui de la FNEGE, pour évaluer la qualité des publications potentielles des candidats. De manière générale, en raison d'un calendrier très contraint, ainsi que d'une augmentation du nombre de candidatures ces dernières années, les évaluateurs sont amenés à accorder de l'importance à des critères tels que les revues dans lesquelles sont publiés les articles ou les types de congrès auxquels les candidats ont participé. En effet, comme souligné par Charle (2009), lire une thèse (et pas seulement son résumé ou son rapport), plusieurs articles ou communications, voire des ouvrages, est un travail long, fastidieux et exigeant qui devient de plus en plus difficile à réaliser, particulièrement par manque de temps, plus encore lorsque l'on se situe dans une discipline autre que celle du candidat (ce qui est le cas de l'un des rapporteurs sur les deux).

\section{Conclusion}

Pour conclure, il semble que la section 06 du CNU ait à cœur de ne pas tomber dans une sorte de "fétichisme " du chiffre, tout en gardant en tête que le chiffre n'est ni bon ni mauvais en soi et qu'il est possible d'en faire une utilisation raisonnable. Le CNU, en tant qu'instance de pairs experts d'une discipline, évalue les dossiers pendant la session de qualification des futurs collègues sur la base de plusieurs critères qualitatifs et quantitatifs. L'un des enjeux de la procédure de qualification est la légitimation disciplinaire et nationale des dossiers soumis. Ainsi, ces dossiers sont reconnus comme ayant une contribution académique à la discipline selon les trois volets de l'activité des enseignants-chercheurs énoncée à l'université française (activité scientifique, activité pédagogique, engagement collectif local et national, scientifique et institutionnel). L'instance CNU n'est pas une chambre d'enregistrement. Actuellement, la section 06 du CNU est composée de 48 pairs (96 si l'on compte les suppléants), qui discutent, débattent et évaluent certainement de façon imparfaite mais de façon homogène, avec comme buts et projets l'intégrité de la discipline et la qualité de la recherche et de l'enseignement des sciences de gestion et du management au sein de l'université française. 


\title{
Évaluer l'évaluation de la recherche
}

\author{
Le cas des enseignants-chercheurs en sciences \\ de gestion et du management
}

Éric SÉvERIN et Jérôme DuPUIS

Contrairement à une idée reçue, les enseignants-chercheurs sont régulièrement évalués. Pour le devenir, ils ont dû convaincre deux rapporteurs et un jury de thèse, passer par la qualification du CNU, là aussi avec deux rapporteurs, convaincre des comités de sélection pour trouver un poste à l'université. Une fois cela fait, l'évaluation continue sur ses enseignements (dans un grand nombre de formations existent des comités de perfectionnement où les étudiants font des retours sur les enseignements officiés), sur la qualité de la formation qu'il dirige au travers de nombreux indicateurs comme le taux d'insertion (pour valider des maquettes de formation il faut faire une auto-évaluation en interne à mi-parcours, prendre en compte l'évaluation de l'HCERES, puis construire une nouvelle maquette qui devra être votée par les instances de l'université avant d'être validée par le ministère). Bien entendu, ce processus se renouvelle régulièrement.

Du côté de la recherche, l'enseignant-chercheur devra faire connaître ses travaux au travers de conférences et journées d'études, et surtout écrire au travers de multiples supports : ouvrages, manuels et, bien entendu, articles dans des revues classées, sans oublier la diffusion de la culture scientifique au travers d'autres supports plus vulgarisateurs. Depuis l'autonomie des universités, un nouveau pas a été franchi sur la recherche. II ne suffit pas d'écrire des chapitres d'ouvrages, des ouvrages, des manuels mais surtout des articles dans des revues classées. Le point de base, si nous pouvons nous exprimer ainsi, est de publier de deux articles en quatre ans. Au travers de ce choix, on observe que l'évaluation est donc largement quantitative à savoir qu'elle est faite en fonction du nombre d'articles publiés en prenant en compte le classement de la revue considérée comme un "benchmark » de qualité. Si on en reste là, c'est la fin de l'histoire et il n'est point besoin de CNU, ou de toute autre instance d'évaluation, pour juger de la recherche (et de sa qualité) d'un enseignant-chercheur. Allons même plus loin et soyons provocateurs pour les besoins du 
chapitre : un simple algorithme guidé par une intelligence artificielle pourrait évaluer les enseignants-chercheurs, et cela avec la plus parfaite objectivité.

L'enjeu n'est pas de déplorer la réalité mais de comprendre pourquoi nous en sommes arrivés là et se poser les questions pertinentes pour que l'évaluation retrouve du sens. Celui-ci est d'importance tant les tensions sont fortes et multiples sur l'activité de recherche et son évaluation (tension institutionnelle pouvant conduire à la normalisation, tension gestionnaire pouvant exacerber la compétition pour mobiliser les ressources, tension organisationnelle pouvant renforcer l'hyperspécialisation, tension culturelle pouvant remettre en cause les finalités mêmes du travail réalisé). Notre propos s'organisera en deux parties. Dans la première, nous chercherons à comprendre les modes d'évaluation (revues indexées, facteur d'impact, $h$ index), leurs limites intrinsèques (périodes considérées, nombre de revues reconnues dans le domaine de spécialité, etc.) et extrinsèques (pratiques de surdensité des auteurs et/ou de citations croisées, etc.), et leurs coûts directs et indirects (relevant du dédouanement, de la justification ou en encore de la non-coopération). La seconde s'attachera, sur la base des rapports les plus récents de qualification ou de promotion des enseignants-chercheurs de la section 06 du CNU, à souligner l'élargissement progressif des registres évaluatifs considérés, pour ensuite, élargir notre champ de vision aux impacts managérial et sociétal qui seront illustrés par des exemples.

\section{L'évaluation : mode de fonctionnement, coûts et dérives}

Évaluer est un exercice difficile. Même si l'on s'adresse à des spécialistes, aucune évaluation n'est pleinement objective car personne n'échappe à la subjectivité et à la jalousie. À l'heure actuelle, l'évaluation se fait essentiellement au travers de revues dites classées par la FNEGE, le CNRS $^{1}$ ou l'HCERES. II ne faut pas oublier que ces classements ne sont que la résultante d'un processus qui remonte en 1958 lorsque qu'Eugène Garfield créé I'Institute for Scientific Information (ISI). Par la suite, plusieurs autres bases ont été constituées comme la Science Citation Index (SCI) en 1963, à présent détenu par Clarivate Analytics, précédemment division de propriété intellectuelle et des affaires scientifiques de Thomson Reuters, et le Social Science Citation Index (SSCI) en 1973, bases initialement dévolues à la recherche bibliographique. Plus récemment, d'autres bases ont été créées : Scopus en 2004 puis, plus récemment, Google Scholar. Les deux premières sont plus étroites (revues académiques) et la troisième prend en compte les conférences.

1 En 2021, le CNRS, dans le contexte national et international de mise en œuvre des politiques publiques de la science ouverte, a pris la décision de renoncer à toute forme de classement de revues en économie et gestion. 
Évaluation du chercheur : revues indexées, impact factor, $h$ index

\section{Classement}

En France, le classement de référence en gestion est celui de la FNEGE, même si la section 06 du CNU ne s'interdit pas de regarder le classement CNRS ${ }^{2}$. En partant de cette base, un bon chercheur est celui qui publie dans des revues classées. Chaque revue rapporte des points (des étoiles) selon son degré d'exigence. Revenons à l'évaluation des chercheurs. Un chercheur doit administrer la preuve qu'il travaille. Il se doit de présenter le fruit de sa réflexion et de sa recherche auprès de sa communauté lors de conférences en expliquant comment il a obtenu ses résultats et, au-delà, les publier lorsqu'ils sont convaincants. Comme l'indiquent Bessire et Fabre (2014), le " publiant devient produisant en recherche ", permettant à cet instrument de gestion, inspiré du new public management, de répondre à trois finalités : une simplification des décomptes (Durand et Dameron, 2011), un pilotage automatique de type calculatoire, et une pression « douce " sur l'activité attendue des enseignants-chercheurs.

Un bon chercheur est donc un chercheur qui publie. Certes, mais pas n'importe où. Il se doit publier dans des revues ad hoc (classées) reconnues par la qualité de leur sérieux au sein de la communauté scientifique. Comment savoir si ces résultats sont valables ? En les lisant et en les étudiant? Ce travail a été fait par les experts de la revue ${ }^{3}$. C'est donc la revue et ses réviseurs qui réalisent le travail d'évaluation. La revue est garante de la qualité des articles. Elle se doit à un exercice sérieux, au risque de perdre sa crédibilité. Tout cela revient à considérer qu'un bon chercheur publie dans de bonnes revues.

Mais alors qu'est-ce qu'une bonne revue? Une bonne revue est une revue indexée car elle figure sur une liste de tel ou tel organisme, organisme qui lui-même est constitué de personnes expertes du domaine. Vient alors une autre question : comment savoir si ce classement est bon ? Simplement car il a été validé par les experts de la discipline. Le système est critiquable et suscite bien des interrogations sur les justifications scientifiques car pourquoi choisir un expert plutôt qu'un autre, d'autant plus que, le plus souvent, leurs noms ne sont pas connus, laissant poindre ainsi une critique d'opacité (Pontille et Torny, 2010). En outre, comme le relèvent Charreaux et Gervais (2007)

un système d'évaluation reposant principalement sur des indicateurs subjectifs est particulièrement vulnérable aux stratégies d'influence de ceux qu'il est censé contribuer à évaluer.

2 Sans revenir sur l'origine des classements, force est reconnaître que le classement de Shanghai privilégie les États-Unis comme celui de Leiden les Néerlandais. Ainsi, prendre le classement de Shanghai pour la France pose tout de suite un problème de contexte.

3 Une revue est souvent organisée autour d'un rédacteur en chef et d'éditeurs associés en fonction de leur domaine de compétence. Une fois une première sélection réalisée, la revue envoie à plusieurs réviseurs le soin de juger de la qualité de la production. Dans les revues les plus prisées (rang 1), le taux de rejet est supérieur à $90 \%$, voire à $95 \%$, taux à rapporter à l'évolution du nombre de soumissions et à affiner au regard de la sélectivité opérée selon le nombre de lectures et révisions. 
Malgré un intérêt indéniable des classements connus de tous ${ }^{4}$, la référence au nombre de publications dans des revues classées, et surtout leurs usages par les enseignants-chercheurs, les institutions académiques comme les média, ont plusieurs effets pervers largement pointés (Osterloh et al., 2008 ; Pontille et Torny, 2010), en particulier en sciences de gestion et du management : la multiplication de la quantité au détriment de la qualité ${ }^{5}$; la focalisation sur les revues (les ouvrages n'étant pas comptabilisés en sciences de gestion et du management, contrairement à d'autres SHS); l'appauvrissement des objets et méthodologies de recherche au détriment de leur diversité ou leur originalité ; I'utilisation de l'addition des publications dans les revues comme note académique. Or, comme on le sait, devant des problèmes complexes, les approches et visions du monde sont différentes. II faut donc trouver autre chose, quelque chose qui soit incontestable. Cet autre chose est l'impact factor (facteur d'impact) ${ }^{6}$.

\section{Impact factor}

Le facteur d'impact de I'ISI est le rapport entre le nombre de citations se référant à une revue sur le nombre d'articles publiés par celle-ci. Ce facteur d'impact est lié à une revue et non à un article. Ainsi, une citation critique d'un article pèse du même poids qu'une citation élogieuse. De fait, on a plus une mesure de popularité qu'une mesure de prestige (Guerrero-Bote et Moya-Anegón, 2012), la citation devenant " monnaie de l'activité scientifique " (Wouters, 1999). Là encore, la démarche et le résultat n'est pas exempt de limites et d'imperfections (Starbuck, 2005).

La première limite est d'augmenter le nombre des auteurs et ou de citer des collègues amis ${ }^{7}$. La deuxième limite est le court-termisme de cette évaluation qui ne prend en compte que les dernières années (deux à cinq ans), ce qui est une fenêtre relativement courte. La troisième limite est qu'il ne prend pas en compte les spécificités des disciplines et ne permet pas comparer en toute objectivité. Les rythmes de publication sont différents entre les disciplines et les facteurs d'impact aussi (le facteur d'impact d'une revue médicale est bien supérieur à celui d'une revue de

4 Y compris à travers leur concurrence stimulante (CNRS, HCERES, FNEGE), et malgré la faible place consacrée aux revues francophones par rapport aux revues de langue anglaise lorsque d'autres classements sont considérés comme celui de l'Econlit (expanded journal list), celui du professeur Harzing (journal quality list), ou encore celle de l'Association of Business Schools (academic journal quality guide).

5 Tout le monde a en tête le cas australien au milieu des années 1990 qui, en adossant le financement de la recherche au nombre de publications dans les revues à comité de lecture, a conduit à en augmenter le nombre alors que le taux de citation diminuait, ce pays passant au dernier rang de I'OCDE (Weingart, 2005).

6 Selon que l'on prenne une méthode ou une autre, il peut avoir divergence. Ainsi, Expert System with Applications est classé FNEGE 3 alors que Decision Support Systems est classé FNEGE 1, alors que même que le facteur d'impact est de 5,45 pour la première et de 4,72 pour la seconde.

7 Pour publier de façon plus productive, des méthodes alternatives peuvent être pratiquées. L'une d'entre elles est le HARKing (hypothesis after results are known). L'idée est simple. Elle consiste, en utilisant des données, de tester des relations explicatives. Lorsque les résultats sont prometteurs, on construit alors la revue de littérature sur ce résultat, ce qui donne un « produit fini » identique à celui d'une démarche scientifique classique. De même, on peut relever la mise au point " industrielle ", dans certaines écoles de commerce, pour la production d'articles, d'une stricte division du travail entre co-auteurs. 
mathématiques) (Aguinis et al., 2020). La force de cet argument est d'autant plus importante en sciences de gestion et du management lorsque le temps de révision avant publication est proche de deux, voire trois ans, renforçant le nombre d'années à considérer. Dès lors, la "fraicheur " ou la portée des analyses peuvent aussi être émoussées ${ }^{8}$.

Enfin, la quatrième limite est l'hyperspécialisation et son corollaire, le manque d'innovation. On parle d'hyperspécialisation car il s'agit de connaître de façon exhaustive un domaine pour mettre en évidence le ou les gaps que le papier s'efforcera de combler; on traite ici d'un sujet connu sur lequel on fait des extensions. Cette méthode est sans doute pertinente dans certains domaines relatifs à l'exploitation des connaissances existantes dans lesquels on teste, on affine et on étend une théorie existante. En revanche, elle est moins pertinente dans les cas où il s'agit de de découvrir un phénomène ou de mettre en évidence une nouvelle théorie (Fisher et Aguinis, 2017). Plus globalement, la mesure en termes de facteur d'impact ne semble s'adresser qu'à la communauté académique, ignorant les autres parties prenantes. D'ailleurs une enquête conduite par Brinn et al. (2000), certes un peu ancienne mais dont les conclusions ne semblent pas être aujourd'hui démenties, auprès d'universitaires comptables britanniques, indique que les évaluations par les pairs sont perçues comme plus importantes que les indices de citations, et ce, quelles que soient l'ancienneté, l'institution et l'activité de recherche des répondants.

\section{Nombre total de citations, $h$ index}

Dans le prolongement du nombre total de citations (NTC), qui repose sur une idée simple, à savoir que si un chercheur est cité, c'est que ses écrits sont intéressants, et face à ses limites, d'autres mesures ou indices ont été proposés. L'une des plus célèbres est le $h$ index, indice appelé $h$ car développé par le physicien californien Jorge Hirsch. Cet indice mesure le nombre de publications et leurs citations. Le $h$ index est égal au nombre de contributions (N) dans la liste de contributions d'un chercheur donné qui ont reçu $\mathrm{N}$ citations ou plus. Ainsi, un $h$ index de 6 de Jean Bombeur, ayant publié 12 articles, signifie que six de ses articles ont été cités au moins six fois, tandis que les articles restants ont tous été cités six fois ou moins. À titre d'exemple, le $h$ index d'Oliver Williamson était de 130 en août 2021, soit 130 contributions au moins citées 130 fois! Mais là encore, on note des limites outre celles déjà citées (nombres d'auteurs ou non prise en compte des différences entre disciplines).

Concernant le $h$ index, la manipulation est possible ${ }^{9}$, comme le soulignent Franceschini et Maisano (2011), ainsi que l'exemple repris par Gingras (2014). Celui-ci rapporte l'histoire vraie d'un informaticien français, Cyril Labbé, qui a mis à jour la façon de manipuler cet indice. Ce dernier a « créé » un chercheur fictif en physique

8 Le recueil et le traitement des données pouvant demander deux ans, la révision deux ans. Une forte obsolescence des conclusions (après quatre à cinq ans), dans un contexte de plus en plus changeant en management, peut alors apparaitre.

9 Cela peut aller, dans certaines écoles de commerce, jusqu'à « investir » la plateforme RePEc (Research Papers in Economics) qui comporte des liens vers de plus de 1,3 million d'articles et working papers classés selon leur notoriété (citations, téléchargements, etc.), et y enregistrer toutes les publications de leurs propres chercheurs et des professeurs affiliés appartenant à d'autres universités pour ainsi grossir le stock d'articles comptabilisés. 
dénommé Ike Antkare. En créant de toutes pièces une centaine d'articles courts se citant mutuellement, et en les plaçant sur un site Internet, ce chercheur fictif a atteint un $h$ index de 94 ! Ike Antkare est devenu l'un des chercheurs les plus cités de l'histoire de la science moderne, et en avril 2010, avec un tel $h$ index il avait largement dépassé celui de la majorité des plus grands chercheurs vivants. Par comparaison, les auteurs les plus cités dans les disciplines les plus porteuses ne voient que très rarement leur $h$ index dépasser les 40 points. Avec un score supérieur à 90, Ike Antkare peut se prévaloir d'une influence sur la science moderne comparable à celle d'Albert Einstein. À ceci près que Ike Antkare n'est pas physicien, mais chercheur en informatique. On l'aura compris, Ike Antkare (prononcer "I can't care ", soit " Je m'en fiche ») n'existe pas, et sa contribution à la science complètement factice. Que tirer de tout cela ? Que ces mesures sont inutiles ? Non, mais imparfaites, certainement. II est facile de critiquer sans proposer de solutions alternatives. Mais avant de faire des propositions, il faut voir les défauts de la cuirasse. Ces défauts s'expriment à travers leurs coûts.

\section{Les conséquences, ou coûts de la mesure}

Les arguments de l'évaluation sont de donner des indicateurs (encore) en vue de pouvoir dresser des diagnostics et de mesurer les points forts et les zones de progrès. Encore faut-il qu'ils soient adaptés à l'appréciation d'une activité complexe comme la recherche (Kirsch, 1996), qu'ils favorisent des comportements proactifs et vertueux, et qu'ils ne dénaturent pas les valeurs de l'organisation dans laquelle elles s'exercent (Simons, 1995). Ce qui est visé, c'est la performance, cette alliance de l'efficience et de l'efficacité. Cela est louable mais a un coût. On peut distinguer des coûts directs et des coûts indirects, ces derniers étant souvent cachés pour la communauté.

Les coûts directs tiennent aux moyens financiers nécessaires à publier dans les meilleures revues. En effet, la recherche en gestion est majoritairement en anglais (Denis, 2015). À moins d'être natif ou parfaitement bilingue, il est donc obligatoire de passer par des relectures faites par des " natifs " (copy editing). Dans certaines disciplines où l'utilisation de bases de données chiffrées est essentielle, il y a aussi obligation de s'abonner à des bases de données reconnues pour des travaux empiriques ${ }^{10}$. Là encore, le chiffrement se fait en dizaine de milliers d'euros. Ces coûts pèsent sur les budgets des laboratoires de recherche, contraints financièrement. Les coûts sont aussi liés aux bases bibliographiques. II faut garder à l'esprit que toute bonne recherche commence par une connaissance approfondie de la littérature, et donc par l'accès aux revues scientifiques. Ces dernières sont regroupées au sein de sociétés ou d'organisation (Elsevier, JSTOR, Wiley, etc.). Ces sociétés sont des sociétés privées ayant pour objectif le profit; elles utilisent leur position incontournable pour le faire. Le principe de révision, hélas pas toujours bien valorisé, repose le plus souvent sur la gratuité. Or, rapporter sur un article prend du temps.

Une fois les obstacles passés, les enseignants-chercheurs "publiants » perdent le plus souvent leurs droits d'auteur. On arrive donc à la situation suivante. Les

10 Il est vrai que certaines écoles de commerce, dans le cadre de leur stratégie explicite de " recherche ", ne prennent pas le temps de vérifier la fiabilité des données en utilisant directement les bases en accès libre comme World Bank of St. Louis Datastream, ou encore Bloomberg. 
enseignants-chercheurs sont encore majoritairement des personnes appartenant à l'université ; par leur statut de fonctionnaire payés par de l'argent public, ils sont les chevilles ouvrières de la connaissance mais ils doivent utiliser les bases bibliographiques pour avoir accès à cette connaissance. Les universités sont donc contraintes de mobiliser des ressources très importantes pour permettre à leurs chercheurs de rester au fait de la connaissance qu'ils ont eux-mêmes contribués à produire. Un exemple connu est celui d’Aron Swartz, décédé en 2013, qui a milité pour le libre accès des connaissances et des savoirs. II a voulu mettre à disposition tous les anciens articles de JSTOR pour lequel le montant des abonnements est très coûteux. Certains de ces articles sont trop anciens pour être protégés par des droits d'auteur, mais les éditeurs l'ont poursuivi en invoquant d'obscurs droits de numérisation.

Outre le fait que ces coûts induisent une inégalité (les chercheurs appartenant à des laboratoires de référence et importants ont un meilleur accès à l'information que les laboratoires de plus petite taille), des coûts indirects existent et sont très conséquents. Le premier coût indirect tient aux coûts de dédouanement, si l'on se réfère à la théorie de l'agence. En effet, avec l'autonomie des universités, une place de plus en plus forte a été donnée aux appels à projet. Ces derniers, s'ils sont sources de ressources financières, génèrent aussi des coûts, à savoir l'obligation de justifier de I'utilisation des fonds (Osterloh et al., 2008). Chacun sait le temps que prend cette justification avec des formulaires et autres fichiers Excel à renseigner. Qu'il soit légitime de montrer que l'argent est bien dépensé est une chose, mais que ce soient les enseignants-chercheurs eux-mêmes qui doivent le faire est sujet à questionnement. En effet, le plus souvent en l'absence de personnel administratif, ce sont les équipes elles-mêmes et leur direction qui sont en charge de ce travail. Lorsque les enseignants-chercheurs font ce qui est appelé - le plus souvent péjorativement - de l'administratif, ils ne se consacrent pas à leur tâche principale, et au cœur de leur métier, à savoir la production de savoir.

Parfois même, les contraintes sont si lourdes que les enseignants-chercheurs renoncent à engager certains projets tant la lourdeur, en amont (réponses aux appels d'offres) et en aval (comptes rendus d'avancement et de bilan), ne leur permet d'avancer comme ils le désireraient, et peut entrainer une perte de motivation, sans parler du faible taux de réussite (16\% en 2019) pour les APP de l'ANR. La motivation extrinsèque (reconnaissance institutionnelle pour avoir " réussi » la conduite à bonne fin de projets de recherche), ou plutôt son absence, peut faire disparaitre la motivation intrinsèque des enseignants-chercheurs pour la valeur propre de leur mission de recherche, l'esprit de curiosité et le partage de savoir découvert dans ce cadre (Cameron et al., 2001). Mais ces coûts indirects ne sont pas les seuls. Le diable est souvent dans les détails. En effet, l'accès aux ressources étant difficile et conditionné par les résultats obtenus, il a pour conséquence une concurrence accrue entre les laboratoires. Si la concurrence a des vertus, poussée à l'extrême, elle conduit à des coûts. Ce sont des coûts de non-coopération alors même que la recherche est fondée sur l'échange et la coopération.

Enfin, il existe un dernier coût indirect plus pernicieux encore : celui de la perte de compétence. En France, les universités délivrent les doctorats. Le système de l'enseignement supérieur en France est dual avec les universités et les " grandes écoles ". Avec la modification du paysage de l'enseignement supérieur et la nécessité des accréditations (AACSB, EQUIS), les écoles de commerce ont dû évoluer. Le corps 
professoral se doit désormais d'avoir validé un niveau scientifique. Les nouveaux enseignants nouvellement recrutés doivent se prévaloir de l'obtention d'un doctorat. C'est là où le coût indirect apparait. Avec des salaires plus élevés et des avantages liés aux primes à la publication, de bons éléments quittent l'université qui les a formés. Le coût de la formation est supporté par l'université et les bénéfices retirés par les écoles de commerce. Même si des partenariats existent, il faut reconnaître que les incitations financières liées à la nécessité de publier défavorisent les universités.

À l'issue de cette démonstration relative au mouvement de " quantophrénie ", pour reprendre Sorokin (1956), le constat semble sans appel. L'évaluation s'est professionnalisée et malgré ses coûts évidents, ce modèle devient le modèle dominant qui ne doit pas être remis en cause. Fin de l'histoire, diront certains. Évidemment non, car on ne peut se satisfaire d'une telle situation imparfaite. Bien sûr, réussir à corriger tous les errements et erreurs est impossible mais il est légitime de s'interroger sur un renouveau de l'évaluation. C'est l'objet de la seconde partie.

\section{Pour une remise en question des démarches d'évaluation}

Les membres du CNU connaissent tous le système de l'intérieur et sont conscients de ses limites. L'instance a vocation à se questionner pour que l'on évalue l'évaluation. D'ailleurs, contrairement à des idées reçues, les membres du CNU jugent en perspective en se donnant du recul et en essayant de donner du sens. Il suffit pour s'en convaincre de lire les rapports d'activités disponibles au sein de la section $06^{11}$. Les perspectives reposent sur deux piliers : la prise en compte déjà engagée des limites et coûts des registres traditionnels de mesure et d'appréciation; l'évaluation des impacts aux niveaux managérial et sociétal de la recherche en sciences de gestion et du management.

\section{Prise en compte des limites et des coûts}

Pour répondre aux limites et aux coûts précédemment mis en évidence, il ressort que, de plus en plus, il est mis en avant la qualité du savoir, et non plus la quantité d'informations. Ceci transparaît à travers la valorisation et la diffusion des connaissances, et non plus la seule et " simple " production d'articles académiques, l'appréciation contextualisée et qualitative des travaux de recherche, et non plus leur simple comptabilisation.

\section{Des « produisants » valorisant et diffusant leurs recherches}

Les enseignants-chercheurs en sciences de gestion et du management se sont attachés, depuis de nombreuses années, à valoriser et à diffuser leurs recherches selon des canaux diversifiés (Starkey et Madan, 2001) : publications par les chercheurs eux-mêmes, ou en collaboration avec des dirigeants d'entreprises, d'ouvrages dans des

11 Il est à noter que la section 06 est l'une des sections qui apporte le plus d'informations à la communauté des enseignants-chercheurs en sciences de gestion et du management : [https://www. conseil-nationaldesuniversites.fr/cnu/], consulté le 13 juin 2021. 
collections académiques ou de plus grande audience ${ }^{12}$ (Carton et Dameron, 2012) ; interventions sous forme d'articles ou tribunes dans des journaux professionnels (comme par exemple dans la revue Échanges devenue en 2013 Finance \& Gestion de la DFCG), ou des journaux mixtes dont les comités de lecture associent académiques et professionnels (comme les Cahiers du Management Territorial), ou encore des lettres électroniques comme The Conversation, dans des webinaires, des émissions de radio ou de télévision spécialisées (comme FNEGE Medias ou Xerfi Canal). Toutes ces initiatives permettant de gagner en lisibilité et notoriété à effets interne et externe (Saint-Onge et al., 2016).

L'intégration des connaissances scientifiques dans l'enseignement constitue aussi un moyen d'en enrichir et d'actualiser les contenus, et par là même, de valoriser et diffuser les résultats issus de la recherche (Rousseau et McCarthy, 2007), en permettant aux étudiants d'appréhender les préoccupations des entreprises comme la relation client, l'innovation, la digitalisation des process, le leadership responsable, la performance globale, la gouvernance éthique, etc. La diffusion élargie peut enfin s'ouvrir à la vulgarisation grand public dans la presse économique ou généraliste (Les Échos, Le Monde, par exemple), ou d'autres médias afin d'assumer pleinement « la concurrence avec d'autres acteurs (journalistes, consultants) opérant sur le marché de la connaissance en management » (Carton et Mouricou, 2017).

\section{Contextualisation et enrichissement de l'évaluation des travaux de recherche}

Il est nécessaire de se concentrer sur la véritable mission de l'université et des enseignants-chercheurs : la création de connaissance, son partage et la diffusion des savoirs, mettant ainsi en évidence que la production de connaissance est intimement liée à sa transmission. Un défi majeur qui mérite d'être relevé en tant qu'enseignant-chercheur, c'est de trouver l'équilibre entre les différentes facettes du métier qui permet de répondre aux besoins des étudiants en leur donnant les connaissances indispensables à leur épanouissement et à leur insertion professionnelle. L'université doit permettre l'émancipation. Cela est pris en compte dans toute évaluation.

Revenons au rapport d'activité de la section 06. Sur la session de mars 2020, il est précisé que pour la qualification aux fonctions de professeur des universités (la procédure dite 46-1)

le CNU a pris en compte explicitement l'implication dans les missions collectives significatives et durables. Aucun des qualifiés de la session 2020 ne présentait un dossier sans responsabilités réelle, que ce soit au plan local, national ou international, au-delà même d'une responsabilité de diplôme généralement présente pour tous les candidats.

12 Notons que le prix Ouvrage de Management FNEGE, en collaboration avec l'European Foundation for Management Development, organisé en partenariat avec les associations scientifiques de gestion présentes dans le Collège Scientifique de la FNEGE, concernant toutes les disciplines de management (marketing, finance, comptabilité / contrôle, GRH, management stratégique, systèmes d'information, entrepreneuriat, logistique, management public), est remis non seulement pour des ouvrages de recherche ou des manuels, mais aussi pour des essais s'adressant à un plus large lectorat. 
De même, dans son rapport sur les avancements des professeurs des universités et des maîtres de conférences de septembre 2020, il est précisé que

les critères retenus sont fondés sur l'exigence de travaux de recherche de qualité attestés par une production reconnue (articles dans des revues classées, communications dans des colloques de référence, ouvrages reconnus, etc.), sous réserve d'un engagement significatif au sein de la communauté " sciences de gestion et du management $"$.

Autrement dit, le CNU va au-delà de la simple évaluation bibliométrique et évalue un profil sous plusieurs facettes, en les contextualisant dans le temps (continuité dans le temps des productions sous différentes formes : articles, working papers, communications dans des colloques nationaux et internationaux, séminaires de recherche), et dans l'espace (considérant avec finesse la taille et la localisation des unités de recherche qui peuvent limiter les échanges entre enseignants-chercheurs sur des champs communs). Cet exemple illustre parfaitement la reconsidération de l'évaluation telle que décrite dans la première partie du chapitre. De même, il est mis l'accent sur l'implication dans les méthodes pédagogiques innovantes.

L'enrichissement de l'évaluation passe aussi par la prise en compte, voire la reconnaissance à sa juste place de la diversité des méthodes de recherche, combinant démarches quantitatives et qualitatives (David, 2004), en particulier à travers la contribution de ces dernières à la mise en lumière de connaissances théoriques, à partir d'une « observation rigoureuse des faits, utiles pour améliorer l'efficacité et l'efficience des décisions managériales [adossée au] concept d'evidence-based management » (Cappelletti, 2009), et aux démarches de recherche-intervention (Cappelletti, 2009), de recherche-action (Greenwood et Levin, 2007 ; Reason et Bradbury, 2008), ou de recherche collaborative-engaged scholarship (Van de Ven et Johnson, 2006).

$\mathrm{Au}$ fond, la question qui mérite d'être posée est de savoir si la multiplication d'articles et de contributions est réellement judicieuse. De grands auteurs en management sont connus pour avoir écrit un article de référence. Il faut aller vers la qualité et permettre à l'innovation de se propager par porosité, sachant que les exigences de publication rendent difficiles de s'aventurer hors des programmes les plus établis. C'est pourquoi il faut favoriser les propositions de numéros spéciaux car ils peuvent être un moyen d'ouvrir les revues à des approches nouvelles. Comment y arriver? La transdisciplinarité demandée - et conseillée - lors des évaluations de laboratoire peut y contribuer. En sortant de sa zone confort, on peut mettre à l'épreuve nos visions du monde et schémas de pensée pour apporter des avancées significatives à la connaissance. Pour cela, il faut que les instances évaluatrices considèrent ces travaux comme des prises de risque payantes à long terme, permettant de sortir du court-termisme.

\section{Impact managérial ( $m$ index) des contributions}

\section{Un relatif divorce entre enseignants-chercheurs et managers-praticiens}

Carton et Mouricou (2017) ont pointé les difficultés de compréhension réciproque entre chercheurs et praticiens en termes d'approches trop mono-disciplinaires développées par les premiers (silos entre stratégie, ressources humaines, contrôle, 
ou focalisation sur des moyennes décontextualisées ou des objets abstraits), et d'écarts d'attentes et de modalités de collaboration pouvant conduire à un faible usage des théories et concepts par les managers alors que ces derniers veulent une approche systémique propre à résoudre des problèmes concrets. Toutefois, l'utilité de la recherche, ne se décrétant pas a priori, mais se constatant souvent a posteriori (Astley et Zammuto, 1992), ne doit pas être uniquement examinée à l'aune de l'opérationnalité immédiate. Elle peut s'exercer de façon plus diffuse et lointaine, et selon des cheminements complexes (Baudet et al., 2016).

Son évaluation peut alors relever de plusieurs utilisations (Denis et al., 2009) : instrumentale (pour la prise de décision et l'amélioration des performances organisationnelles), processuelle (pour le changement de perceptions et représentations des acteurs sur une problématique), conceptuelle (pour la transformation de paradigme dans la conduite des organisations), ou habilitante (pour le changement de pratiques et le développement de nouvelles capacités organisationnelles et managériales). Enfin, le recours à des concepts ou des théories ne permet pas forcément d'améliorer la pratique (Sutton et Staw, 1995). Certains d'entre eux pouvant relever du mimétisme, de la répétition décontextualisée, de l'effet de mode, ou encore conduire à des effets pervers (comme le lean management) ou, dans le meilleur des cas, contrastés (comme pour la RSE ou le design management).

\section{Des réponses déjà apportées à des fins d'usage}

Les réponses relèvent de trois registres principaux : connaitre, reconnaitre, fertiliser, comme en témoigne notamment le numéro spécial de la Revue française de gestion (novembre-décembre 2016) consacré à l'impact de la recherche. La connaissance passe par une meilleure identification des préoccupations des praticiens professionnels à laquelle contribue, depuis 2013, le Baromètre FNEGE des attentes des entreprises. Ce dernier « a pour objectif de comprendre les principales préoccupations des entreprises et de faire le lien avec les thématiques des enseignants-chercheurs ". Il contribue, comme d'autres dispositifs ou observatoires, à définir les métiers émergents ou les transformations des métiers traditionnels (en particulier en matière de digital en marketing, ou suite à l'introduction de l'intelligence artificielle dans nos disciplines [finance, contrôle, ressources humaines] et thématiques [logistique, santé, gestion publique]), de nature à adapter les offres de formation ${ }^{13}$ et à venir explorer de nouveaux terrains de recherche.

La reconnaissance a pour objet de contribuer à valoriser les productions formalisées et distanciées (aspects "instructif, original, utile et inspirant ») de managers par le monde académique comme le fait le prix $M \& M$ décerné par La maison du management et la FNEGE au meilleur ouvrage de management écrit par un manager. Enfin, la fertilisation consacre les nombreuses passerelles pouvant naître entre recherche et pratique : projets communs et partenariats, capitalisation partagée sous forme de comptes rendus d'expériences décryptés et analysés, mise à disposition

13 Voir par exemple le référentiel prospectif des compétences " Management et gestion des affaires ", sous la direction d'Aline Scouarnec, FNEGE/ AUNEGe, juillet 2019. 
de guides pratiques issus de la recherche ou de "savoirs modélisés ${ }^{14}$ ", publications portant un double regard sur un concept une théorie ou le décryptage d'un cas empirique, temps de rencontres croisés enseignants-chercheurs / managers ${ }^{15}$. On peut aussi noter dans ce cadre l'importance donnée, dans les critères d'évaluation des enseignants-chercheurs par la section 06 du CNU, à l'investissement personnel dans les associations académiques reconnues, car la section est consciente de leur rôle majeur dans le croisement des regards entre chercheurs et praticiens. Celles-ci viennent opportunément, à travers leurs ateliers, colloques ou webinaires, fertiliser les réflexions et pratiques des professionnels et leurs besoins.

\section{Le $m$ index à des fins de preuve d'utilité}

Sur ces bases, Kalika (2018) propose un outil de mesure et d'appréciation de l'impact managérial de la recherche, le $m$ index, se déclinant aux différentes étapes de son processus. Sont alors à examiner : « les échanges conduisant à la fertilisation croisée des approches susceptibles d'améliorer les conceptions des chercheurs et des praticiens " lors de la définition du projet; " l'impact sur les organisations résultant des réflexions des chercheurs " lors de son exécution; "l'impact sur les pratiques des gestionnaires " lors de la diffusion des résultats; l'impact en termes de méthodes d'infusion (séminaires, groupes de travail) au sein des organisations lors de la mise en œuvre des résultats. Ensuite, pour chaque phase (au nombre de quatre), cinq critères viennent apprécier l'intensité de l'item, chacun d'eux étant noté de 0 à 5 . La cotation peut alors être calculée sur 100 points ( $4 \times 5 \times 5$ ). Kalika (2018) imagine une utilisation du $m$ index pour un projet de recherche, une publication, un chercheur (indice $m$ moyen par auteur) et « également être utilisé pour cartographier les publications et les chercheurs sur un bidimensionnel montrant la publication de l'impact académique et managérial ».

L'intérêt d'une telle approche est de spécifier les différentes formes d'impact managérial, de les justifier par des preuves qualitatives et quantitatives, et d'en définir le poids global et relatif, venant par là même enrichir l'évaluation académique (comptabilisation des productions, notoriété issue des citations), et surtout prouver l'utilité managériale. Nous pouvons enfin, pour illustrer les aspects saillants de ces protocoles d'identification de l'impact managérial des travaux de recherche, prendre appui sur l'un de nos contrats de recherche-action et publications afférentes (encadré 1).

14 Comme le fait l'ISEOR, ayant conçu une méthode d'accompagnement des entreprises et des organisations pour améliorer leur performance sociale et économique durable et qui, à ce titre, a formé plus de 2000 dirigeants, managers ou consultants.

15 Comme s'y est attaché l'Association pour la Recherche et l'Expertise en Management Public Territorial, fondée par Jérôme Dupuis et Joseph Carles, en partenariat avec les associations de cadres et dirigeants territoriaux. Elle a organisé, de 2003 à 2012, les Universités d'été du management territorial sur des thèmes comme la décision publique, la gestion des risques, l'innovation publique, la régulation publique, ou encore le management par le sens et les valeurs dans les collectivités locales. 


\section{Encadré 1 : Exemple emblématique d'impact managérial d'une recherche-action : Partie I}

Un contrat de recherche-action (2013-2015) entre l'IAE de Lille et le ministère du Développement durable a porté sur l'élaboration d'un cadre d'évaluation des valeurs générées par les territoires d'exception (Grands sites de France [GSF] et sites inscrits au Patrimoine mondial [PM] de l'Unesco), mettant en lumière les processus (à travers des questions évaluatives et des critères qualitatifs) et les résultats (à travers des indicateurs chiffrés et un baromètre critériel sur chaque volet économique, social, environnemental et paysager, et territorial) des actions de protection et de développement menées, des moyens mobilisés sur ces territoires et des modes opératoires en termes de gouvernance territoriale. Les travaux se sont adossés à des enquêtes de terrain sur cinq sites (trois GSF et deux PM) et ont donné lieu à la rédaction de cinq monographies (2013), un guide-référentiel (2015), cinq communications dans des colloques académiques, et trois contributions à un ouvrage collectif.

Quatre types d'impact peuvent être notés. Le premier impact est un usage instrumental à travers la confection par le ministère d'un document "Comment évaluer les valeurs économique, sociale, environnementale et territoriale ? Une méthode opérationnelle pour les acteurs des territoires d'exception " (2016), qui lui sert tout autant pour qualifier les dossiers sollicitant une labellisation, aux équipes de gestion des sites d'exception pour valoriser leur création de valeurs et améliorer les réalisations et résultats de leurs programmes d'action, et aux équipes projets sollicitant une reconnaissance comme Grand site de France ou une inscription au Patrimoine mondial de I'UNESCO pour argumenter leurs candidatures. Le deuxième impact est un usage conceptuel en ne limitant plus l'approche évaluative à une production d'indicateurs quantitatifs, puisque chaque valeur fait l'objet de quelques questions évaluatives adossées à des objectifs stratégiques et de critères de mesure et d'appréciation quantitatifs et qualitatifs. Un troisième impact est un usage processuel en ayant fait partager l'idée de trajectoires différentes de développement des sites qui prennent en compte les contextes locaux, en particulier lors des 20e Rencontres du réseau des grands sites de France de 2018). Enfin, un quatrième impact est un usage habilitant en promouvant, grâce au référentiel mettant en exergue facteurs clés de succès, points de vigilance, exemples et Retex, les capacités de coproduction entre acteurs et parties prenantes.

L'impact managérial se retrouve dans les méthodes désormais en vigueur : au sein du ministère et des réseaux nationaux de ses partenaires (réseau GSF, Association des biens français du patrimoine mondial) ; dans l'évolution des pratiques des gestionnaires (compétences en matière d'évaluation et capacité au partage d'expériences); dans l'appropriation de ce type de démarche par les décideurs politiques (en particulier grâce aux baromètres synthétiques permettant de rendre compte de la gestion des sites et de leurs projets de territoires, de justifier les investissements publics mobilisés mais aussi de débattre collectivement, à différentes échelles, sur des bases tangibles et vérifiables, des réussites et pistes de progrès) ; et, plus globalement, dans la connaissance plus outillée des mécanismes et preuves de création de valeur patrimoniale et de développement durable, ainsi que des tensions et équilibres entre préservation / restauration vs. valorisation / attractivité.

L'exemple relaté dans l'encadré 1 prouve que la recherche est de nature à contribuer à des impacts concrets pour les bénéficiaires susceptibles d'être directement concernés et, que dans le cas d'espèce, notre discipline, à savoir le management public, et plus largement les sciences de gestion et du management, peut aussi venir alimenter les contenus de l'action politique et des politiques publiques. C'est ainsi qu'il nous faut désormais élargir et affiner le champ de vision de l'impact de la recherche en sciences de gestion et du management pour aborder la question de l'impact sociétal. 


\section{Impact sociétal des contributions}

\section{Importance et enjeux de l'impact sociétal}

La question de l'impact sociétal, intentionnel ou non, avéré ou non, intéresse tout autant les enseignants-chercheurs à titre individuel, que les instances académiques, les acteurs socio-économiques et les décideurs politiques. Pour les premiers, il permet de donner du sens au métier en tant que fonctionnaires d'État visant l'intérêt général. La connaissance disciplinaire mobilisable par la société, ou irrigant celle-ci d'un savoir utile pour de nombreuses parties prenantes qui dépassent les frontières académiques, permet de justifier l'importance singulière des sciences de gestion et du management pour l'amélioration du fonctionnement des organisations et des systèmes de décision qui s'y rattachent (Schoemaker, 2008). Le caractère innovant de cette connaissance peut aussi être perçu comme une source de création de valeur socioéconomique pour les acteurs des territoires. Quant aux décideurs politiques, l'appréciation des effets des savoirs en gestion et management peuvent contribuer à éclairer les enjeux de société et à alimenter les politiques publiques susceptibles d'y répondre (Fulconis et al., 2016).

Son évaluation soulève toutefois plusieurs difficultés. Les principales sont de deux ordres. La première porte sur des dimensions dont les caractéristiques qualitatives et multifacettes sont fortes, et qui ne prennent leur sens que sur le long terme. La seconde questionne la place donnée aux objectifs et impacts attendus qui pourraient constituer le point d'appui et de référence à toute appréciation de la sorte. Or, ces aspects sont rarement explicites au départ des projets de recherche; ils se découvrent au fur et à mesure de leur réalisation, voire nécessite un travail particulier d'analyse.

\section{Qualification de l'impact sociétal}

Malgré ces difficultés, il nous faut pouvoir avancer pour répondre à trois questions intéressant chaque partie prenante évoquée plus haut : quelle est la création de valeur publique du " savoir ", du projet de recherche et des publications afférentes (quoi ? Dans quel but et pour quelle finalité ?) ; quels sont les mécanismes venant la soutenir (comment? Quels en sont les facteurs clés de succès ?) ; quelle en est sa répartition (au profit de qui ?). Pour cela, nous proposons de nous focaliser sur trois catégories d'impact : l'impact scientifique cumulatif (Penfield et al., 2014), qui conduit à de nouvelles initiatives de recherche de nature à affiner le projet initial dans son contenu et ses méthodes; l'impact institutionnel et politique (Meagher et al., 2008), qui introduit un " recadrage " des politiques publiques ouvrant de nouvelles stratégies ou modifiant les voies et moyens antérieurement utilisés; I'impact relationnel (Pettigrew, 1987 ; Starkey et Madan, 2001), qui permet de conforter ou d'imaginer de nouveaux mécanismes de participation et de coopération entre les acteurs.

L'intégration de ces impacts dans les protocoles de recherche et leur déploiement est de nature à favoriser la capacité des chercheurs à questionner les effets sociétaux de leur recherche et à contribuer à la résolution des problèmes à l'interface entre management et société tels que la croissance soutenable, la responsabilité sociale, l'innovation, etc., celle des sponsors publics et privés à orienter leurs financements, 
celle des institutions et décideurs publics à sélectionner des projets de recherche porteurs d'effets sociaux et politiques et à mettre en débat leurs conclusions avec la société civile, celle de l'ensemble des parties prenantes à s'engager collectivement dans la voie de la « recherche coproduite " (O'Hare et al., 2010). Cette piste de réflexion, adaptée aux sciences de gestion et du management, a donné lieu à la mise en place par la Commission européenne du Social Impact Open Repository.

Le lecteur peut s'interroger sur l'absence dans notre propos de l'impact socioéconomique tel que promu par le Research Excellence Framework ou les Business School Impact Survey et University Impact System (Lejeune et al., 2019).La justification que nous avançons est double : d'une part, ils ne portent pas exclusivement sur des projets de recherche ou l'activité recherche des enseignants-chercheurs, mais sur des institutions d'enseignement supérieur consolidant les volets formation-recherche-administration et gestion ; d'autre part, il nous semble très complexe d'isoler l'effet quantitatif propre de projets de recherche tant les effets liés sont nombreux. C'est pourquoi nous avons privilégié les trois impacts présentés ci-dessus, en insistant en particulier sur les logiques de coopération et de valorisation locale et supra-locale des savoirs qui mettent en exergue les effets positifs de la proximité et de la mise en réseau (Baudet-Michel et al., 2020). Nous pouvons désormais, pour illustrer la tentative d'appréciation de l'impact sociétal, tel que défini plus haut, poursuivre l'exemple évoqué précédemment (encadré 2).

\section{Encadré 2 : Exemple emblématique d'impact managérial d'une recherche-action : Partie II}

L'impact scientifique cumulatif peut se retrouver dans le lancement de trois nouveaux programmes de recherche PUCA affinant et poursuivant nos conclusions par le ministère de l'Écologie, du Développement durable et de l'Énergie. C'est le cas, par exemple, de celui consacré aux "Sites exceptionnels : quelle contribution au développement local ? Les sites exceptionnels comme ressources des territoires ", confié à une équipe constituée de chercheurs appartenant à trois structures différentes, deux laboratoires de recherche et un bureau d'études (UMR Pacte-Grenoble ; Lab'Urba-Paris-Est ; Acadie-Reflex). Remis en juillet 2017, il a contribué à « analyser les processus par lesquels ces (inter)dépendances se construisent, les ordres de tensions auxquels ces processus conduisent, en fonction des sites, des territoires dans lesquels ils s'inscrivent et des dimensions en jeu ». Ces travaux viennent aussi, les uns et les autres, enrichir l'école de pensée qui promeut l'approche par les valeurs publiques de la Public Value Management.

L'impact institutionnel et politique se produit lorsque les résultats publiés et diffusés ont été transférés dans une politique publique. Dans notre cas, un premier séminaire de recherche PUCA (juillet 2017) a posé les bases d'un diagnostic et de propositions pour apprécier les incidences liées aux limitations d'accès aux sites, les conséquences sur le plan économique de la perte d'accessibilité et de la juste mesure permettant d'assurer la préservation du site dans son intégrité, tout en ouvrant l'accès au plus grand nombre. En outre, le Sénat s'est saisi de cette problématique à travers le rapport n 110 du 13 novembre 2019 de Jérôme Bignon fait au nom de la commission de l'aménagement du territoire et du développement durable et portant sur le régime général d'accès aux espaces naturels et aux sites bénéficiant d'un régime de protection en raison de leur dimension esthétique, écologique ou culturelle. Une proposition de loi ( $\left.n^{\circ} 2437\right)$ a tenté de traduire cela en permettant au maire de réglementer, et non plus seulement d'interdire, l'accès et la circulation des personnes. Celle-ci, votée au Sénat le 21 novembre 2019, a été transmise à l'Assemblée nationale qui, en avril 2021, ne l'avait toujours pas inscrit à son ordre du jour. 
Notre travail de recherche initial a montré comment la labellisation permettait la création d'un réseau d'acteurs mobilisés ou la consécration d'initiatives, même anciennes, légitimant le territoire. Dans cet esprit, et éclairant l'impact relationnel ainsi consacré, les nouvelles candidatures comme Grand Site de France ou Patrimoine Mondial de I'UNESCO ont conforté la dynamique de collaboration élargie et participative sur le terrain entre institutions, acteurs socio-économiques, associations, habitants résidents, touristes, etc., associant des organismes de recherche du territoire comme l'ont montré les cas du GSF Solutré-Pouilly-Vergisson (apport du programme de recherche du laboratoire ThéMA 8 de l'Université de Bourgogne) et de l'inscription des Climats de Bourgogne au Patrimoine mondial de l'UNESCO, mobilisant un comité scientifique de 36 spécialistes : géographes, géologues, climatologues, historiens, sociologues, linguistes, biologistes et ingénieurs agronomes, qui a su renforcer l'argumentaire sur la valeur universelle exceptionnelle du territoire.

Cet exemple prouve que la recherche est de nature à contribuer également, sur un temps plus long et plus difficilement repérable, à des impacts sociétaux dont les spécifications peuvent s'apprécier avec modestie. Les savoirs se construisent dans une logique plus proche de la tectonique des plaques tectoniques que de la généalogie : un mouvement ne disparaît pas au profit d'un autre mais reste vivant, parfois sous d'autres formes. Ainsi, dans notre cas d'espèce, le public value management ne vient pas se substituer au new public management mais ils cohabitent en compagnie aussi de la new public governance. L'aspect scientifique cumulatif se découvre dans les débats, les controverses ou les disputes argumentées (au sens étymologique du terme, disputare : mettre au net après examen et discussion).

Les paramètres de la décision politique sont parfois éloignés des registres scientifiques, et notre exemple a montré comment les considérations sur les impacts dommageables à l'environnement liés à la sur-fréquentation des espaces et des sites naturels et culturels fragiles pouvaient prendre du temps à se traduire dans l'action, et parfois se réduire à de simples ajustements réglementaires. La mise en évidence de l'aspect relationnel n'est possible qu'au prix d'observations fines et qualitatives. Cependant, nous avons pu remarquer que la préexistence de réseaux et de relations avec les utilisateurs de la recherche, comme l'activation de multiples intermédiaires dans la diffusion du savoir, permettent la génération d'impact à ce niveau.

\section{Conclusion}

Le comptage et l'intelligence artificielle censés faire ressortir un résultat n'est pas d'actualité au CNU, qui privilégie l'intelligence humaine, jusqu'à présent la seule capable de bien " évaluer " ! L'évaluation est difficile, et la raison en est simple. Les outils développés dans ce qu'il convenu d'appeler la bibliométrie n'arrivent pas à prendre en compte les spécificités de chaque domaine et des méthodes utilisées. C'est la raison pour laquelle, si ces outils restent utiles, il faut aussi développer des actions plus qualitatives dont les contours restent à préciser ou à construire, tant pour le plan " micro " considérant les interactions entre le chercheur et les destinataires directs et utilisateurs potentiels de ses productions (l'impact managérial), que pour le plan « méso " considérant les relations avec les acteurs collectifs et les 
institutions dans et hors le milieu académique (l'impact sociétal ${ }^{16}$ ). Ceci passerait par la capacité à éclairer et à justifier nos réponses à quelques questions comme : dans quelle mesure les productions de recherche, sous leurs différentes formes, permettent-elles une diffusion, une valorisation ou un usage au-delà du cercle académique? ; quels sont les exemples concrets d'utilisation instrumentale, conceptuelle, processuelle et habilitante que les praticiens, au sens large de terme, sont susceptibles de traduire ? ; les impacts scientifiques cumulatifs, institutionnels et politiques relationnels sont-ils avérés et selon quelles modalités; quels sont les facteurs clés de succès et les difficultés repérées dans le cadre du déploiement des résultats des recherches et de leurs conditions de production de nature à générer des "flows of knowledge leading to research impacts " (Meagher et al., 2008) ?

Dans cette perspective, nous considérons que c'est à la communauté scientifique, réunie dans sa diversité au sein de la section 06 du CNU, qu'il revient de déterminer le référentiel approprié (en co-production avec un panel élargi de représentants des parties prenantes), de le porter à connaissance et d'évaluer, en premier ressort, chaque situation - sur la base d'une " auto-évaluation » telle que proposée et illustrée dans notre chapitre mais dont les critères sont connus de toutes et tous - pour éclairer les choix des instances académiques locales, et certainement pas de laisser le localisme, gagné souvent par les petits arrangements dans une grande opacité, faire œuvre d'évaluation du travail réalisé par les enseignants-chercheurs en sciences de gestion et du management. Notre contribution tente bien modestement de prendre la mesure et de comprendre les différentes facettes des impacts à court et moyen terme (les outputs comme les outcomes) générés par les recherches en sciences humaines (Ochsner et al., 2016), en les adaptant aux sciences de gestion et du management. En effet, nous sommes conscients que l'évaluation des impacts dans notre domaine n'est pas un long fleuve tranquille, ce que confirment les travaux les plus anciens comme les plus récents sur ce point (Bornmann, 2013). La question reste toutefois ouverte des conséquences (en termes de carrière, de reconnaissance sous toutes ses formes, de moyens attribués, etc.), susceptibles d'être tirées de ces appréciations.

En guise de touche finale, on peut avancer, en rebondissant sur notre titre, " qu'évaluer » est un sport complet. Un bon évaluateur doit bien maîtriser les outils bibliométriques qu'il utilise, mais aussi comprendre les spécificités des disciplines et le contexte dans lequel la recherche se réalise. Rappelons-nous la remarque de Jean-Marc Monteil, président de l'AERES, qui affirmait qu'

il conviendrait, c'est bien le moins, que dans l'évaluation par les pairs, on n'oublie pas que l'évaluation la plus scientifique la plus sérieuse et la plus objective réclame que l'on lise les papiers sur lesquels on porte un jugement.

Sans cela il y a une perte de sens. Or, c'est bien cette recherche de sens qui est le cœur du CNU car aucune mesure ne peut rendre compte de l'obstination d'un chercheur, du temps passé et des sacrifices consentis. Cette recherche de sens ne se limite d'ailleurs pas à la seule production scientifique mais aussi à l'ensemble des activités des enseignants-chercheurs. Nous en sommes tous convaincus.

16 Le volet « macro » n’a pas été approché ici compte tenu des limites évoquées en termes de variables exogènes difficiles à mettre sous contrôle, de liens de causalité et d'imputabilité plus souvent énoncés que démontrés, des particularités contextuelles et culturelles venant relativiser les conclusions évaluatives (Bornmann, 2013). 



\title{
Vers la reconnaissance de la valeur du don Un parcours au sein de la section 06 du CNU
}

\author{
Nicolas Aubert, Marc Bonnet, Aude Deville et Anouk Grevin
}

\begin{abstract}
Mon hypothèse est que les usages philosophiques potentiels du verbe " reconnaître » peuvent être ordonnés selon une trajectoire partant de l'usage à la voix active à l'usage à la voix passive. Ce renversement de plan grammatical porterait la trace d'un renversement de même ampleur au plan philosophique. Reconnaître en tant qu'acte exprime une prétention, un claim, d'exercer une maîtrise intellectuelle sur le champ des significations, des assertions significatives. Au pôle opposé de la trajectoire, la demande de reconnaissance exprime une attente qui peut être satisfaite seulement en tant que reconnaissance mutuelle, que celle-ci reste un rêve inaccessible ou qu'elle requière des procédures et des institutions qui élèvent la reconnaissance au plan politique (Ricœur, 2004).
\end{abstract}

Le présent chapitre vise à discuter l'importance et la valeur du don à l'université en général et, plus particulièrement, au sein du CNU. Le don de celui qui reçoit et qui a reçu d'une communauté, notamment en termes de formation, et qui donne à son tour. Un tel don souffre de non-reconnaissance car souvent l'intérêt calculé y est opposé. Dans une première section sont brièvement présentées les théories du don, et dans une seconde section est développé un parcours du don au sein de la section 06 du CNU.

\section{Les théories du don en sciences sociales}

La littérature sur le don est considérable : Marcel Mauss, Alain Caillé et Jacques Godbout en sociologie, Jacques Derrida, Jean-Luc Marion et Simone Weil en philosophie, George Akerlof, Stefano Zamagni et François Perroux en économie. En sciences de gestion et du management, nous pouvons citer les travaux suivants : Barel et Frémeaux (2008), Alter (2009), Frémeaux et Michelson (2011), Pihel (2011), Grevin (2013), Masclef (2013), Aubert (2016), ainsi que l'ouvrage collectif de Gomez et al. (2015), labellisé par la FNEGE en 2016, entièrement consacré à ces questions. 
L'ouvrage collectif d'Ide et al. (2021) approfondit la question de la réception du don au travail dans la même lignée que celle de Gomez et al. (2015). Pour évoquer les théories du don, nous nous appuyons en particulier sur les contributions de Frémeaux et al. (2014), Gabellieri (2014) et Grevin (2015).

\section{Don pour des philosophes et don échange des sociologues}

Les travaux académiques sur le don sont très nombreux car animés par un débat sur l'existence même du don. L'auteur le plus connu est sans aucun doute Mauss (1923/2012). II a initié les recherches sur la question et la logique donner-recevoir-rendre. La logique de Mauss (1923/2012) consiste à s'écarter de celle de l'échange marchand comme motif de l'action humaine. Si l'on devait situer les logiques de l'échange marchand et du don sur un axe en fonction des motivations de l'action, on associerait sans doute aux deux extrémités du segment la logique du don au désintéressement, d'une part, et celle de l'échange marchand à l'intérêt, d'autre part. C'est une approche anti-utilitariste qui en découle, approche qui a d'ailleurs donné son nom au M.A.U.S.S., Mouvement anti-utilitariste en sciences sociales, et à la revue du même nom dont Alain Caillé est l'un des fondateurs.

Cette approche suppose donc une véritable " révolution du don ", comme l'ouvrage de Caillé et Grésy (2014) nous y invite. Les philosophes Jacques Derrida et Jean-Luc Marion cherchent à identifier les conditions de possibilité d'un don pur, un don dénué de toute recherche d'intérêt. Gabellieri (2014) identifie chez Jacques Derrida trois caractères du don pur : (1) il ne doit pas y avoir de réciprocité, c'est-à-dire de retour possible du don ou de contre-don ; (2) il n'y a don que si l'on ne cherche jamais à rendre le don; et (3) il n'y a don que si le donateur est inconscient de son don. Gabellieri (2014) exprime ainsi l'aporie du don des philosophes : " Ou il existe et contredit son intention de pureté, ou il n'apparaît pas, et ne peut exister ». Un don conscient ne peut pas être pur. Pour Jacques Derrida, le seul don réel donc est anonyme et impersonnel. Le don de temps présente ces caractéristiques selon Gabellieri (2014) :

Donner le temps (ou le recevoir simultanément au don de l'être) c'est donner (ou recevoir), non pas un objet que l'on pourrait rendre, mais la condition de tous les dons et de tous les échanges possibles.

Les conditions posées par les philosophes sont si restrictives que leur analyse du don en limite considérablement la portée dans le champ social. Le don pur est ici caractérisé par le désintéressement total.

Ce n'est pas tout à fait la perspective suivie par les sociologues comme Alain Caillé. $\mathrm{Au}$ don désintéressé s'oppose le don social plus en ligne avec la perspective maussienne, plus « modeste » mais aussi plus réaliste du point de vue d'Alain Caillé. Selon Gabellieri (2014), le don des philosophes ne connait ni l'échange ni la réciprocité, alors que l'échange et la réciprocité caractérisent au contraire le don des sociologues. Il existerait une opposition fondamentale entre don pur et don maussien, deux approches qu'Hénaff (2012) a tenté réconcilier. Gabellieri (2001) évoque une troisième entrée intéressante pour comprendre les dynamiques du métier 
d'universitaire : le don pluriel $^{1}$. Pour sortir de l'opposition don pur / don échange, il introduit un tiers dans la relation en prenant pour exemple la relation de filiation dans laquelle les parents qui donnent la vie n'ont pas d'espoir de retour équivalent. Les enfants ne peuvent rendre le don à leur parent mais ils peuvent donner la vie à leur tour, dans une logique de transmission. Le don initial est sans retour, il suscite de nouveaux donateurs dans une dynamique qui dépasse le schéma duel. D’après Gabellieri (2014), le don pluriel implique d'analyser le don en amont et en aval. En amont, le don pluriel renvoie " à la commune dépendance de tous à l'égard d'une origine à la fois familiale, sociale et métaphysique (si l'on élargit la question au niveau de l'origine ultime de la donation) ". En aval, le don pluriel " renvoie à la multiplication d'un lien entre personnes où jamais le don ne peut être fixé et résolu dans un retour au donataire $»$.

\section{Reconnaître le don au travail}

En sciences de gestion et du management, le don a été étudié dans plusieurs contextes mais nous nous intéressons en particulier à l'étude du don dans un contexte de travail. À la suite d'Alter (2009), Grevin (2013, 2015) identifie le don comme se situant au cœur de la différence entre le travail prescrit et le travail réel, qui a été qualifiée différemment selon les auteurs : activité de régulation, travail d'organisation, travail d'articulation, discussion sur le travail. Le temps et les ressources consacrés individuellement et collectivement à cette activité d'adaptation des règles sont très importants d'après Alter (2009), et relèvent du don au travail. Cette différence entre travail prescrit et travail réel s'incarne en particulier dans l'ingéniosité et le travail de régulation déployés par les travailleurs pour réaliser des tâches concrètes qui ne figurent pas dans une fiche de poste ou un cahier des charges :

$\mathrm{Ni}$ obligatoires ni codifiés, ingéniosité et travail de régulation représentent bien un cadeau, un don que les opérateurs destinent au bon fonctionnement de leur métier ou de leur mission mais, également, au bon fonctionnement de l'entreprise (Alter, 2009).

Pour reconnaître le don, il faut tout d'abord l'identifier. Or, nous l'avons vu, ce qui relève du don ne fait même pas l'objet d'un accord parmi les spécialistes. Pour les philosophes, le don, s'il est vu, serait comme dénaturé car occasionnant une dette et un retour potentiel qui le transformerait en échange. Leurs contributions nous ont cependant permis d'esquisser une opposition entre don pur et don échange, que Frémeaux et al. (2014) synthétisent dans la figure 1, proposant un continuum avec quatre orientations théoriques. On retrouve bien aux deux extrémités le don pur et le don échange. Entre ces deux pôles, les auteurs situent deux notions également issues de la littérature importante sur le don : le don relationnel et le don gratuit.

1 Gabellieri (2014) cite Bruni (2010) pour une extension plus large de ce type de réflexion. 


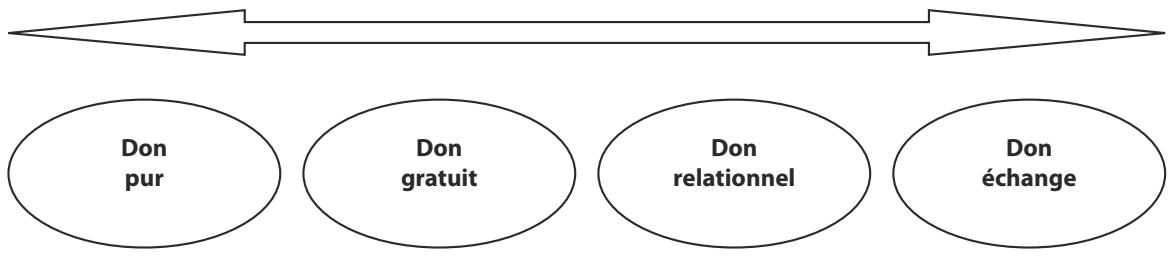

Figure 1 : Différentes conceptions du don. Source : Frémeaux et al. (2014).

Le don gratuit permet d'appréhender l'aspiration humaine naturelle à donner. L'attention est ici portée sur le donateur et non sur ce qui est donné. Le retour peut exister dans ce cas mais il n'est pas la dimension principale. Frémeaux et al. (2014) indiquent que Titmuss (1970/2018) et Malinowski (1922/2014) sont les pionniers de cette approche. Le don relationnel a pour vocation de créer une relation et d'engendrer un cycle de dons. S'il y a une réciprocité, elle engendre une dynamique relationnelle : "Le retour n'est pas forcément attendu par le donneur comme dans le don échange mais, en même temps, n'est pas forcément évacué comme avec le don gratuit " (Frémeaux et al., 2014). Frémeaux et al. (2014) identifient enfin les éléments de convergences et de divergences entre ces différents dons dans le tableau 1.

Tableau 1 : Les éléments de divergence des théories du don

Source: Frémeaux et al. (2014)

\begin{tabular}{|c|c|c|c|c|}
\hline & Don pur & Don gratuit & Don relationnel & Don-échange \\
\hline Définition & $\begin{array}{l}\text { Le don est fait } \\
\text { sans attendre } \\
\text { de contrepartie, } \\
\text { sans intérêt } \\
\text { ni mobile }\end{array}$ & $\begin{array}{l}\text { Le don est fait } \\
\text { sans attendre } \\
\text { de contrepartie } \\
\text { mais a un intérêt } \\
\text { intrinsèque pour } \\
\text { le donneur }\end{array}$ & $\begin{array}{l}\text { Le don peut } \\
\text { générer une } \\
\text { réciprocité } \\
\text { et donc une } \\
\text { dynamique } \\
\text { relationnelle } \\
\text { même quand le } \\
\text { don initial est un } \\
\text { don gratuit }\end{array}$ & $\begin{array}{l}\text { Le don appelle } \\
\text { un contre-don. } \\
\text { II s'opère dans } \\
\text { une double } \\
\text { ambivalence } \\
\text { " liberté / } \\
\text { contrainte " } \\
\text { et " gratuité / } \\
\text { intérêt " }\end{array}$ \\
\hline Angle d'analyse & Donneur & Donneur & Relation & Échange \\
\hline $\begin{array}{l}\text { Rapport à la } \\
\text { réciprocité }\end{array}$ & $\begin{array}{l}\text { Le don pur } \\
\text { n'est jamais } \\
\text { un don } \\
\text { réciproque }\end{array}$ & $\begin{array}{l}\text { Le don gratuit } \\
\text { peut être un don } \\
\text { réciproque, mais } \\
\text { la réciprocité } \\
\text { n'est pas une } \\
\text { composante de la } \\
\text { définition du don }\end{array}$ & $\begin{array}{l}\text { Le don } \\
\text { relationnel, } \\
\text { tout en étant } \\
\text { inconditionnel, } \\
\text { vise une relation } \\
\text { de réciprocité }\end{array}$ & $\begin{array}{l}\text { Le don / contre- } \\
\text { don est } \\
\text { instrumental et } \\
\text { vise le retour }\end{array}$ \\
\hline $\begin{array}{l}\text { Intention } \\
\text { première } \\
\text { du donneur }\end{array}$ & $\begin{array}{l}\text { Faire un don } \\
\text { absolu }\end{array}$ & $\begin{array}{c}\text { Exaucer un } \\
\text { besoin de donner }\end{array}$ & $\begin{array}{c}\text { Créer une } \\
\text { dynamique de } \\
\text { don }\end{array}$ & $\begin{array}{c}\text { Générer une } \\
\text { obligation de } \\
\text { retour }\end{array}$ \\
\hline
\end{tabular}

Dans le contexte de la section 06 du CNU, centrée sur les sciences de gestion et du management, la responsabilité de notre discipline ne se limite pas à l'analyse de concepts issus d'autres disciplines issues des autres sciences sociales, des 
mathématiques et de l'informatique. En effet, les sciences de gestion et du management sont des sciences de la décision et de l'action collective. Comme pour les autres sciences de l'action telles que les sciences de l'ingénieur et les sciences médicales, une mission importante est de proposer des méthodes et des outils ayant des impacts positifs pour nos parties prenantes. Dans le cadre du présent chapitre sur le don, nous proposons ainsi de nous appuyer sur des concepts issus de la philosophie pour expérimenter un outil d'évaluation du don dans le cadre des missions du CNU.

\section{Don ou travail prescrit dans le cadre du CNU?}

Les règles de la fonction publique amènent à considérer implicitement que la participation aux instances nationales est un don gratuit, faisant partie d'une activité non prescrite dans le temps réparti entre la recherche, l'enseignement et les responsabilités collectives conçus par le législateur. Aucune décharge de service n'est accordée pour les membres du CNU. II existe cependant une indemnité d'un montant brut annuel moyen de 1000 euros par membre du CNU siégeant au cours de toutes les sessions d'une année. Ce montant comprend une partie fixe partagée entre le titulaire et le suppléant, et une partie variable calculée en fonction du nombre de dossiers traités. L'indemnité ne peut pas être considérée comme une véritable rémunération car elle sert principalement à couvrir des faux frais liés à l'activité du CNU : compte tenu du niveau irréaliste des normes de remboursement des déplacements dans la fonction publique, il s'agit notamment des surcoûts de déplacement non remboursables. Une telle indemnité correspondant à la rémunération d'un cours d'une vingtaine d'heure ne couvre évidemment pas les heures de travail ni la responsabilité engagée.

II serait naïf de considérer que l'activité de membre du CNU correspond à un don pur. Toutefois, le manque de transparence sur la nature du don introduit un doute dans l'esprit des collègues, qui sont aussi électeurs des deux tiers des membres du CNU, le dernier tiers étant nommé par le ministère. Des suspicions - parfois légitimes - de la part des collègues sont exprimées : "Un tel ou une telle va au CNU pour favoriser sa promotion, soit au cours de la mandature actuelle, soit dès le début de la mandature suivante ", ou encore : "Ce collègue favorise les membres de son groupe d'appartenance ou de sa spécialité ", et bien d'autres. En interne, au CNU, les débats seraient pollués par des non-dits sur les objectifs implicites de collègues ou de listes ne relevant pas du bien commun ni du don pur, mais ayant des visées de conquête de pouvoir ou électoralistes.

Une telle suspicion est bien sûr malsaine car elle peut jeter un certain discrédit sur la mission de régulation et de garde-fou du CNU face aux risques très élevés de dérives du localisme. En effet, le contexte français implique que les universités restent majoritairement financées par l'État au niveau national, avec une relative autonomie pour les dépenses, mais en ayant une très faible responsabilisation sur leurs ressources. II y a, en outre, une quasi-absence de mesure des impacts sur les parties prenantes qui financent les universités. II en résulte que les systèmes de gouvernance et de management des universités sont souvent critiqués pour leur manque de professionnalisme et pour leur clanisme, sous couvert de démocratie et d'indépendance. En dehors des contrôles de conformité de la Cour des comptes et des évaluations académiques par I'HCERES, les universités françaises n'ont pas encore de comptes à rendre à la société qui les finance (Bourdieu, 1984, 1989). 
L'exigence croissante de transparence et les crises dans la société et dans l'économie française vont probablement remettre en question les institutions universitaires et les contraindre à justifier davantage chaque activité. Cette tendance va dans le sens de l'exigence de responsabilité sociétale et elle est amplifiée par le développement des réseaux sociaux. L'opacité des décisions sur les carrières n'est plus acceptable, notamment au niveau local, en raison des dons échanges sur les postes et les promotions. II convient par conséquent de réfléchir à la nature des dons et à leurs contreparties, en s'appuyant notamment sur le modèle proposé dans la figure 1 par Frémeaux et al. (2014).

\section{Nature du don}

À défaut de pouvoir créer une image fidèle et purement comptable des dons dans le cadre de l'activité du CNU, il convient d'établir des rubriques relatives à ce que l'on donne, à qui l'on donne, aux impacts de ce qui est donné, et aux rétributions qualitatives, quantitatives et financières. À défaut également d'une évaluation qui reste à mener à bien à réaliser, il est proposé d'identifier les apports et rétributions de chaque partie prenante dans le cadre d'un travail d'évaluation de la performance durable. Il s'agit justement d'un domaine de recherche pour lequel la profession d'enseignant-chercheur en sciences de gestion et du management peut légitimement apporter sa contribution à des débats idéologiques sous couvert d'objectivité scientifique.

\section{Don à la communauté par les membres du CNU}

Les travaux à exécuter sur une mandature de quatre ans comprennent principalement l'évaluation d'une centaine de dossiers de qualification (qui vont être en partie remplacés par des dossiers de suivi de carrière à l'avenir), une soixantaine de dossiers de promotion et une cinquantaine de dossiers de PEDR. II faut y ajouter, jusqu'en 2020, l'examen de quelques dossiers de qualification au titre de l'article 46-3 (accès à la fonction de professeur des universités par la "voie longue »). Cela représente donc environ 200 dossiers à examiner sur quatre ans, soit une cinquantaine par an. En supposant que le titulaire et le suppléant se partagent équitablement la charge de travail, chaque membre du CNU - ou son suppléant - va donc examiner une centaine de dossiers au cours de la mandature. Chaque examen de dossier demande au minimum deux heures de travail car il s'agit d'un enjeu important pour la carrière des collègues. Nous pouvons observer que les collègues du CNU sont, pour la plupart, conscients de l'importance des responsabilités de l'évaluation, d'autant plus que leur réputation de professionnalisme est en jeu.

Le travail à réaliser comprend les opérations suivantes : (1) première lecture du dossier ; (2) vérification des données, par exemple en ce qui concerne les publications ; il est parfois nécessaire de réorganiser le dossier lorsque les classements des revues ne figurent pas ou sont erronés (par exemple, oubli de mention du classement FNEGE) ; (3) rédaction d'une fiche d'instruction du dossier en rappelant le parcours du candidat et ses réalisations de matière d'enseignement, de recherche et de responsabilités administratives; et (4) deuxième lecture du dossier pour vérification 
et rédaction d'un commentaire de synthèse, et attribution d'une note pour chaque critère et d'une note globale. Par ailleurs, il est nécessaire d'assister en présentiel à trois sessions du CNU par an, une pour les qualifications, une pour les avancements et une pour l'attribution des PEDR. Cela représente au total une dizaine de journées de sessions par an, avec des déplacements à Paris ou en province. Chaque journée d'une dizaine d'heures de travail comprend à la fois des présentations de dossiers et des temps de discussion en cas de nuances d'évaluation entre les deux rapporteurs, mais aussi des échanges d'informations et des débats sur les critères d'évaluation des dossiers.

Il faut ajouter une mission connexe de représentation du CNU dans un comité HCERES pour évaluer les unités de recherche relevant de la section 06. On peut formuler l'hypothèse d'une évaluation par mandature pour chaque membre du CNU, ce qui comprend une cinquantaine d'heures de travail pour instruire le dossier, participer à la visite HCERES et contribuer à la rédaction du rapport. À mi-parcours de la mandature 2019-2023, les collègues ont traité 1895 dossiers. La répartition en est présentée dans le tableau 2. On pourrait aussi mentionner le travail d'animation des listes dans le cas des membres du CNU élus, les sollicitations fréquentes pour participer à des jurys de thèse ou de HDR, mais surtout, la charge de travail considérable de présidence du CNU, ainsi que celle des membres du bureau. Le don de temps par un membre du CNU au cours d'une mandature correspond ainsi à l'équivalent de trois mois de travail pour un professeur des universités. Les membres du CNU appartenant au corps des maîtres de conférences ont une charge de travail correspondant en moyenne aux deux tiers de celle des professeurs car il y a un peu moins de dossiers à examiner et un moindre temps de participation aux sessions (tableau 3). En termes de coûts d'opportunité, un tel temps aurait pu permettre la rédaction d'un article dans une revue classée ou encore une rémunération de l'équivalent de 250 heures supplémentaires de cours.

Tableau 2 : Nombre de dossiers évalués à mi-parcours de la mandature 2019-2023

\begin{tabular}{|c|c|c|c|}
\cline { 2 - 4 } \multicolumn{1}{c|}{} & $\begin{array}{c}\text { Professeurs } \\
\text { des universités }\end{array}$ & $\begin{array}{c}\text { Maîtres } \\
\text { de conférences }\end{array}$ & $\begin{array}{c}\text { Total (sans les dossiers } \\
\text { communs) }\end{array}$ \\
\hline $\begin{array}{c}\text { Promotions } \\
\text { (2020 et 2021) }\end{array}$ & 386 & 352 & $649^{(\text {a) }}$ \\
\hline PEDR (2020 et 2021) & 183 & 121 & 304 \\
\hline $\begin{array}{c}\text { Qualification (2020 et } \\
\text { 2021) }\end{array}$ & 942 & 795 & $942^{(\text {b) }}$ \\
\hline Total & 1511 & 1268 & 1895 \\
\hline
\end{tabular}

Notes : (a) En enlevant 89 dossiers en commun; (b) En enlevant 795 dossiers en commun. 
Tableau 3 : Hypothèses de temps de travail en heures d'un membre du CNU au cours d'une mandature

\begin{tabular}{|c|c|c|c|c|}
\hline \multirow[b]{2}{*}{ Missions } & \multicolumn{2}{|c|}{ Professeurs des universités } & \multicolumn{2}{|c|}{ Maîtres de conférences } \\
\hline & $\begin{array}{l}\text { Temps de travail } \\
\text { sur une } \\
\text { mandature } \\
\text { de quatre ans } \\
\text { (en heures) }\end{array}$ & $\begin{array}{l}\text { Hypothèse } \\
\text { de répartition } \\
\text { équilibrée } \\
\text { entre membre } \\
\text { du CNU, titulaire } \\
\text { et suppléant }\end{array}$ & $\begin{array}{l}\text { Temps de travail } \\
\text { sur une } \\
\text { mandature } \\
\text { de quatre ans } \\
\text { (en heures) }\end{array}$ & $\begin{array}{l}\text { Hypothèse } \\
\text { de répartition } \\
\text { équilibrée } \\
\text { entre membre } \\
\text { du CNU, titulaire } \\
\text { et suppléant }\end{array}$ \\
\hline $\begin{array}{l}\text { Dossiers de qualification } \\
\text { (PU : } 200 ; \text { MCF : 100) }\end{array}$ & 400 & 200 & 266 & 133 \\
\hline $\begin{array}{l}\text { Dossiers de promotion } \\
\text { (PU: } 60 ; \mathrm{MCF}: 30)\end{array}$ & 120 & 60 & 80 & 40 \\
\hline $\begin{array}{l}\text { Dossiers de PEDR } \\
\text { (PU : } 50 ; \mathrm{MCF}: 25)\end{array}$ & 100 & 50 & 66 & 33 \\
\hline $\begin{array}{c}\text { Sessions } \\
\text { (PU : } 10 \mathrm{j} \text {./an } \times 4 \text { ans } \\
\text { MCF : } 5 \mathrm{j} \text {./an } \times 4 \text { ans) }\end{array}$ & 400 & 200 & 266 & 133 \\
\hline $\begin{array}{l}\text { Comités HCERES } \\
(\mathrm{PU}: 2, \mathrm{MCF}: 1)\end{array}$ & 100 & 50 & 66 & 33 \\
\hline Total & 1120 & 560 & 740 & 370 \\
\hline
\end{tabular}

À qui donne-t-on et pour quels impacts?

Plusieurs parties prenantes bénéficient du travail des membres du CNU, dont les principaux sont les suivants : l'État, la profession, les collègues et les membres du CNU eux-mêmes. Examinons rapidement chacune d'elles.

\section{L'État (ministère et universités)}

Officiellement, le don de plus de 500 heures de travail par membre du CNU doit profiter au ministère de l'Enseignement supérieur et de la Recherche qui, par ailleurs, y consacre des moyens limités : malgré des progrès encore nécessaires, la plateforme informatique Galaxie permet la dématérialisation des dossiers et le secrétariat du ministère fait preuve d'efficacité et de dévouement. Les réunions sont le plus souvent accueillies dans les composantes avec leurs propres moyens. On peut estimer à moins d'un million d'euros par an la valeur des prestations et indemnités allouées par le ministère et les universités pour faire fonctionner la section 06 du CNU, tandis que le travail bénévole de l'ensemble des membres de la section représente un investissement immatériel d'au moins 1,2 million d'euros : sur la durée d'une mandature, le travail bénévole représente plus de 40000 heures, qui correspondrait, si cela devait être payé, à l'équivalent de six postes en équivalent temps plein.

Le ministère bénéficie ainsi de travaux d'expertise complémentaires des évaluations locales pour améliorer la qualité des processus de recrutement ou de promotion. Rappelons qu'il suffit d'éviter une dizaine d'erreurs par mandature pour déjouer les effets pervers et des coûts cachés considérables, au sens de Savall et Zardet (1987/2020), liés à l'iniquité d'un recrutement lorsqu'un collègue incompétent est recruté ou promu localement par les établissements, et sans prendre en 
compte en outre les impacts désastreux d'un tel recrutement sur des générations d'étudiants et sur l'image et la réputation des universités. Pour les universités, la présence d'une évaluation nationale apporte la chance d'éviter la tentation de la médiocrité, même si cela contrarie les clanismes et les jeux d'influence locaux. Sans être le seul élément du don, le travail du CNU est par conséquent l'un des ingrédients nécessaires au bon fonctionnement du système universitaire français.

\section{La profession}

La section 06 du CNU est une institution qui permet d'assurer un minimum de cohésion d'une profession éclatée entre de nombreuses spécialités et méthodologies de recherche. Compte tenu du très fort sous-encadrement des sciences de gestion et du management par rapport à d'autres disciplines (moins de $24 \%$ de professeurs des universités, alors que la moyenne nationale, toutes disciplines confondues, est de l'ordre de $40 \%$ ), les enseignants-chercheurs y sont très investis dans la direction de diplômes et dans les activités pédagogiques et administratives, ce qui laisse peu de temps pour le dialogue interne à la profession. Sans cette cohésion, la profession serait dispersée par les forces centrifuges venant de disciplines connexes : droit, sociologie, sciences économiques, informatique, etc.

Avec la FNEGE, et parfois avec la SFM sur certains sujets, la section 06 du CNU permet ainsi un dialogue constant entre disciplines, d'autant plus que chaque dossier est systématiquement examiné par un spécialiste du champ et par un spécialiste d'un autre champ : par exemple, les dossiers de spécialistes en finance d'entreprise sont également évalués par des enseignants-chercheurs en GRH ou en stratégie. On se situe ici principalement dans le don relationnel : la profession donne de son temps pour exister au service d'une science de l'action indispensable pour maintenir une prospérité durable du pays dans un contexte de mutations et de complexité croissantes.

\section{La communauté des enseignants-chercheurs}

Le temps donné par le CNU est une garantie pour l'indépendance dont tout universitaire a besoin pour innover. On imagine mal les régressions dont souffriraient les collègues et la qualité de leur recherche s'ils étaient évalués uniquement par les responsables locaux, en étant réduits à consacrer une bonne part de leur énergie, aux frais de l'État, dans des jeux politiques de leur institution. Les méthodes d'évaluation au niveau national des impacts attendus du travail des collègues restent bien sûr à perfectionner. Ces méthodes sont pourtant indispensables pour prévenir les tentations d'une démission de l'État face aux pressions résultant de l'autonomie des universités. Concernant les membres du CNU eux-mêmes, leur don correspondant à l'équivalent de trois mois de travail sur la durée d'une mandature, une explicitation des contreparties à partir de la typologie des dons proposée par Frémeaux et al. (2014) est possible :

- Don pur ou gratuit. Une partie de l'activité de membres du CNU relève de la pure générosité. Les collègues en retirent la joie et la fierté de participer à une profession dont le pays et la communauté internationale attendent beaucoup compte tenu des souffrances liées aux situations de mauvaise gestion ou de " gestion toxique » des entreprises et organisations.

- Don relationnel. La raison d'être du métier d'enseignant-chercheur est à la fois de transmettre ce que l'on a reçu (enseignement) et de le perfectionner et le 
transformer (recherche) pour mieux servir le bien commun et les générations futures. En outre, la création de relations entre collègues et l'examen de dossiers variés permet de mieux appréhender des problématiques du métier en élargissant le cadre local dans lequel les enseignants-chercheurs en science de gestion et du management passent la plus grande partie de leur temps.

- Don-échange. La tentation d'utiliser sa participation au CNU pour bénéficier de contreparties existe, il ne faut pas le nier. On observe aussi des inégalités dans les comportements : par exemple, certains membres sont très méritants, mais ils s'auto-censurent sans déposer leur dossier d'évaluation. Les risques de déséquilibre entre don et contre-don sont donc réels, même s'ils sont limités par la qualité du fonctionnement de la section 06 du CNU, dont le bureau est de nature multipartite.

Une réflexion sur l'amélioration de l'ensemble du système d'évaluation est nécessaire, en articulant mieux les dispositifs pour permettre une évaluation à 360 degrés. Cela doit impliquer toutes les parties prenantes : communauté scientifique nationale du CNU, instances locales, utilisateurs et financeurs de la recherche au niveau local, national et international en prenant en compte de façon globale les impacts scientifiques, managériaux et sociétaux des activités d'enseignement et de recherche.

\section{Proposition d'expérimentation d'une grille d'auto-évaluation}

Dans l'état actuel des universités et de leur tutelle ministérielle, marqué par un manque de clarté stratégique et une grande complexité organisationnelle, il n'est pas aisé de proposer un projet ambitieux de refonte des systèmes d'évaluation des carrières. Pourtant, les enjeux sont cruciaux, non seulement pour notre profession d'enseignants-chercheurs en sciences de gestion et du management, mais aussi pour les universités et pour le pays. II est donc important que la section 06 puisse prendre des initiatives pour au moins deux raisons. D'une part, c'est une question de crédibilité de la section, composée d'experts en management des organisations : nous pouvons seulement nous appuyer sur nos compétences et notre cohésion pour justifier de notre existence, n'ayant pas l'ancrage institutionnel et relationnel d'autres sections plus anciennes, comme celles des juristes, politologues et économistes. D'autre part, c'est un service à apporter au ministère et aux universités car le développement des universités en France ne peut réussir pleinement sans les sciences de gestion et du management.

\section{Le dilemme don / échange au cœur du métier d'enseignant- chercheur en sciences de gestion et du management}

Le métier d'enseignant-chercheur comporte différentes composantes dont les logiques sont souvent difficilement conciliables ${ }^{2}$. Un enseignant-chercheur assure la double mission de produire et de transmettre le savoir. À ces missions s'ajoutent

2 Le métier d'enseignant-chercheur est défini par plusieurs textes : la loi n84-52 du 26 janvier 1984 sur l'enseignement supérieur, le décret n84-431 du 6 juin 1984, la loi n²007-1199 du 10 août 2007 relative aux libertés et responsabilités des universités, et le décret n²009-460 du 23 avril 2009. 
officiellement, depuis 2009, les fonctions relatives au management des formations, des unités de formation et de recherche, des unités de recherche, des établissements. Toujours depuis 2009, les enseignants-chercheurs doivent également assurer l'insertion professionnelle des étudiants. Depuis la loi relative aux libertés et responsabilités universitaires de 2017, le métier est mieux appréhendé par la dénomination enseignant-chercheur-manager (Sacriste, 2014).

II résulte une dialectique propre au métier d'enseignant-chercheur entre logique du don et logique de l'échange, désintéressement et recherche de l'intérêt. Une logique n'est pas supérieure à une autre, a priori. Le réalisme conduit même plutôt à retenir que ces deux logiques cohabitent et que leur équilibre doit être maintenu. Le maintien de l'engagement des universitaires est en effet tributaire du maintien d'un certain équilibre entre les deux logiques, équilibre qui se retrouve dans chacune des trois grandes composantes du métier d'enseignant-chercheur. L'équilibre entre les logiques du don et de l'échange rend nécessaire l'évaluation nationale par des pairs, déconnectée des seules logiques hiérarchique et locale.

Le métier d'universitaire consisterait donc à occuper trois fonctions, et non pas une seule. Aux missions habituelles de production et de transmission des savoirs s'ajoutent des responsabilités collectives, sachant que ce que recouvrent ces responsabilités collectives, et même leur dénomination, ne fait pas l'unanimité au sein de la communauté universitaire. On parle tantôt de responsabilités administratives, tantôt d'engagement collectif, tantôt de responsabilités collectives. Les différentes dénominations ne sont pas neutres et reflètent leur plus ou moins grande valorisation selon les disciplines. Ces responsabilités sont nombreuses et leur contenu est très hétérogène. On peut classer au sein de la rubrique des missions locales et nationales. On peut mentionner les autres fonctions citées explicitement par les textes, telles que les directions d'établissements, de composantes et d'unités de recherche. Les fonctions de directions résultent majoritairement d'une élection et l'on peut donc également citer les mandats électifs. À ces directions sont associées les directions adjointes, les vice-présidences, les responsabilités de départements ou d'axes de recherche. S'y ajoutent les responsabilités scientifiques dans des sociétés savantes, les revues à comité de lecture nationales et internationales.

À côté des sociétés savantes se sont constitués des réseaux pédagogiques. Là encore, les responsabilités sont déclinées. La logique de l'évaluation par les pairs suppose également de mobiliser l'engagement collectif des universitaires pour évaluer les établissements, les unités de recherche, les formations, les carrières individuelles. Ces responsabilités collectives font l'objet d'une évaluation par le CNU, au niveau national, et les établissements, au niveau local. Les critères d'évaluation aux niveaux national et local sont différents car les logiques sont différentes. Au niveau des établissements, les critères d'évaluation et les systèmes d'incitation qui en découlent sont souvent la conséquence de disparités importantes sur le territoire. En France cohabitent en effet des établissements qui figurent parmi les meilleures universités au monde et des établissements qui garantissent l'accès du plus grand nombre à l'enseignement supérieur. Au niveau national, le CNU cherche à développer des critères d'évaluation pour une même discipline sur l'ensemble du territoire tout en veillant à préserver l'équité dans ses évaluations. Au niveau des établissements, les disciplines cohabitent et les stratégies peuvent naturellement conduire à favoriser certaines d'entre elles. Les mêmes logiques locale et nationale se retrouvent pour 
l'évaluation de la recherche et de la formation. En fonction des caractéristiques des établissements, de la représentation disciplinaire en leur sein, la recherche et la formation seront évaluées différemment, alors que l'on retrouvera nécessairement une plus grande homogénéité au sein d'une discipline au niveau national.

\section{Conclusion}

Les sciences de gestion et du management contribuent à former près de $20 \%$ des étudiants de l'enseignement supérieur, avec des taux d'insertion professionnelle remarquables. Elles apportent aux universités des liens qui leur manquent avec les entreprises et les organisations. Elles sont nécessaires au succès du développement des autres disciplines présentes dans les universités pour manager les institutions, les projets de recherche et gérer l'interaction entre les processus de création et d'innovation. Ni plus ni moins que tout travailleur, les universitaires sont donc confrontés à différents critères et se trouvent dans un arbitrage permanent entre logique du don et logique de l'échange. C'est davantage l'hétérogénéité entre les différentes missions qui singularise le métier d'universitaire. En son temps, Weber (1919/2002) a pleinement saisi ces tensions, qu'il traversait sans doute lui-même. À cette dialectique interne s'ajoute l'opposition entre l'éthique de la responsabilité et l'éthique de conviction qui distinguent respectivement la vocation de savant de la vocation de politique.

L'exercice du métier d'enseignant-chercheur-manager conduit les universitaires à des conflits internes qui pourraient confiner à une certaine schizophrénie, même si les attentes et les besoins de reconnaissance des enseignants-chercheurs qui s'impliquent sont légitimes. Qualification, promotion et attribution de primes relèvent d'une réciprocité relevant du don-échange. Une telle dynamique de réciprocité fondée sur la reconnaissance du travail anime l'engagement et fait vivre la communauté. Mais l'exercice du métier d'enseignant-chercheur est aussi motivé par des motifs désintéressés qui relèvent aussi du don gratuit et relationnel, qui ne demandent pas être reconnus. Rendues visibles dans le quotidien de la vie des établissements, ces logiques de don s'évanouiraient sans doute en s'inscrivant dans des logiques de dons contre dons. Selon nous, la reconnaissance de ces engagements par une instance nationale, indépendante des réalités hiérarchiques locales, les maintient et les stimulent.

L'évaluation par les pairs élus et nommés d'une instance nationale comme le CNU est une spécificité française qui découle du statut de la fonction publique des enseignants-chercheurs. Cette évaluation nationale permet d'identifier et de valoriser les apports des enseignants-chercheurs à leurs communautés locales et nationales en favorisant un certain équilibre entre les différentes logiques. Les contributions des membres du CNU à l'évaluation de leurs collègues sont, elles aussi, traversées par des dynamiques de don. Force est d'admettre que de telles contributions sont difficiles à rationaliser compte tenu du temps qui leur est consacré. 


\section{L'évaluation de carrière des enseignants-chercheurs

\author{
Origines, enjeux et perspectives
}

Jean-François SatTin et Sophie Cros

L'évaluation de la carrière de l'enseignant-chercheur est un sujet qui revient régulièrement parmi les objectifs de notre tutelle et ne peut s'apprécier que dans une mise en perspective du contexte historique. Du suivi de carrière au dossier unique les voies proposées sont multiples et suscitent de nombreuses interrogations dans la communauté universitaire. Le présent chapitre fournit d'abord des éléments de compréhension des enjeux sous-jacents à l'évaluation individuelle, ce qui nécessite de replacer l'apparition du débat actuel dans le contexte plus général d'évolution de l'enseignement supérieur. II présente ensuite les dispositifs de suivi de carrière tel qu'il est actuellement proposé, et tel qu'il se dessine pour l'avenir, en en soulignant à la fois la portée et les limites pour l'ensemble des disciplines, et notamment pour les sciences de gestion et du management.

\section{Le développement de l'évaluation individuelle dans I'université française : une mise en contexte historique}

La mondialisation et la massification de l'enseignement supérieur, l'apparition du new public management, le développement des outils numériques et l'introduction de la contractualisation dans l'enseignement supérieur ont eu une incidence profonde sur la façon dont a été façonnée l'évaluation des enseignants-chercheurs. Elle n'a toutefois pu émerger qu'une fois les statuts des universitaires et les grandes institutions universitaires entièrement stabilisés. 


\section{La structuration et l'autonomisation croissante de l'université française}

Les révolutionnaires de 1789 , précurseurs dans la promotion du new public management, stipulaient que " la société a le droit de demander compte à tout agent public de son administration " (article 15 de la Déclaration des droits de l'homme et du citoyen du 26 août 1789). Ils ont fait disparaître les universités existantes, avec peu d'effets sur les collègues qui exerçaient à l'époque (Mérindol, 2008). Si le Premier Empire a réhabilité l'université, la III République a mis en place un système d'évaluation des universitaires à la fois collégial et réalisé par les pairs. L'université impériale, telle qu'elle est prévue dans le décret du 17 mars 1808, définit le rôle des inspecteurs des universités qui, avec les recteurs et les inspecteurs d'académie, sont chargés de contrôler les activités des universitaires. Ce principe va être très vite remis en cause.

Le décret de 1885 et la loi du 10 juillet 1896 vont fixer l'essentiel de l'organisation des facultés et figer le statut des universitaires (Mérindol, 2008). Les corps d'enseignement sont organisés autour des enseignants de rang magistral (professeurs titulaires de chaire, professeurs titulaires à titre personnel, et professeurs sans chaire, maîtres de conférences) et les positions auxiliaires (chefs de travaux pratiques, de chefs de laboratoires de recherches, puis assistants et maîtres-assistants). Les modes d'évaluation des universitaires et de leurs activités, quant à eux, se stabilisent alors autour de trois principes fondamentaux qui resteront en vigueur jusqu'au milieu des années 1980 : (1) l'évaluation est par principe individuelle, les évaluations collectives restent plus marginales ; (2) l'évaluation doit reposer uniquement sur le jugement des pairs ; (3) l'évaluation est réalisée à la demande de l'intéressé, elle n'est ni régulière ni systématique. Il n'y a aucune injonction pour les collègues à se soumettre à une évaluation, réalisée uniquement lors de l'entrée dans la carrière ou lors des demandes de promotions.

Ces principes vont rester en vigueur durant presque un siècle, sans être contestés. Une première brèche va toutefois être ouverte, en 1968, à travers la critique des mandarins et de leurs enseignements, jugés trop éloignés de leur public estudiantin. L'autonomie des universités apparait alors comme une revendication forte de la période (Schnapp et Vidal-Naquet, 1969/2018; Mérindol, 2008). Elle sera reprise dans la loi Faure en 1968, accordant une autonomie renforcée aux universités et créant un " conseil universitaire " auquel participent des étudiants, des personnels enseignants et non-enseignants, ainsi que des personnalités extérieures. La remise en cause des universitaires de rang magistral dans le prestige de leur fonction laissera des traces ${ }^{1}$.

Dans les années 1970, le gouvernement est confronté pour la première fois aux conséquences de la massification de l'enseignement supérieur et au développement de l'emploi précaire à l'université. Les assistants et les vacataires sont très nombreux. Ils conservent des perspectives de carrière limitées malgré la création d'emplois

1 Le SNESUP, qui a vu son importance croître lors des évènements de mai-68, et qui comprend alors plus de $80 \%$ d'enseignants auxiliaires, avance alors, pour la première fois, la revendication d'un corps unique des enseignants du supérieur. II s'oppose à ce niveau aux syndicats autonomes, qui comprennent alors très peu de précaires, et qui défendent le système en place. Le point sera repris par le SGEN-CFDT dans les années 1980. 
de maîtres-assistants entre 1962 et 1968 pour en titulariser certains ${ }^{2}$. Comme le remarque Mérindol (2010)

la proximité d'âge entre les jeunes assistants, recrutés en nombre dans les années 1960 , dont les perspectives de carrière sont incertaines, et les étudiants, eux aussi de plus en plus nombreux et provenant de classes sociales de plus en plus diverses, ne pouvait que faciliter leur rapprochement, et corrélativement l'éloignement avec les professeurs.

Les années suivantes sont marquées par des tentatives de reprise en main des universités par la tutelle, qui se caractérise par un interventionnisme plus marqué, à l'encontre de l'autonomie obtenue précédemment. Cette période se distingue aussi par des tentatives à la fois pour augmenter le pouvoir des enseignants de rang magistral dans les conseils et pour évincer les assistants et les vacataires de l'université. Elle est marquée par des luttes syndicales actives qui conduiront, après 1981, à stabiliser les statuts des enseignants du supérieur autour de deux corps d'universitaires : un corps des professeurs des universités, créé en 1979, dans lequel sont reclassés tous les enseignants du supérieur de niveau magistral (professeurs et maîtres de conférences), et un corps des maîtres de conférences issu de celui des maîtres-assistants, avec un plan d'intégration des enseignants auxiliaires et mise en extinction progressive du corps des assistants.

Le processus aboutit au décret du 6 juin 1984, encore en vigueur en 2022, maintes fois amendé depuis, qui accroît et annualise les obligations de services des universitaires à $128 \mathrm{~h}$ de cours magistraux (192 h équivalent TD) ${ }^{3}$, et qui précise le rôle du Conseil supérieur de l'université dans la gestion des carrières des enseignants du supérieur (dénommés pour la première fois enseignants-chercheurs), en gardant pour référence les trois principes énoncés plus haut. À ce titre, il est intéressant de constater que l'augmentation du service des universitaires semble décidée, au moins pour partie, sur la base de préoccupations budgétaires, et non pas au vu de leurs missions (Mérindol, 2008).

Par ailleurs, le décret du 6 juin 1984 introduit pour la première fois la possibilité de moduler les services des enseignants-chercheurs, dans une fourchette comprise entre 0,5 et 1,5 de leur service de base, en fonction du degré de participation aux autres missions de l'intéressé (recherche et responsabilités administratives). Les présidents d'université sont relativement frileux face à une telle mesure, qui leur semble difficile à mettre en œuvre en l'absence de dispositifs d'accompagnement $\mathrm{RH}$ au niveau local (évaluation systématique des enseignants-chercheurs, notamment), et en raison des contextes électoraux de leurs établissement. D'après Jean-Jacques Payan, cité par Mérindol (2008), c'est la CPU qui fait alors pression sur la tutelle pour que la modulation ne puisse se faire qu'avec l'accord des intéressés ! Les temps ont bien changé... Cette disposition, qui rend la modulation à la hausse inopérante en pratique, sera finalement supprimée en 1988, avant de revenir en force en 2009 dans le cadre de la LRU.

2 Ils doivent être titulaires d'une thèse de doctorat de $\|^{\mathrm{e}}$ cycle et inscrits sur une liste d'aptitude établie par le Comité consultatif des universités.

3 La référence à 1607 heures de travail effectif sera introduite en 2009 par la LRU. 


\section{L'émergence de l'évaluation individuelle dans les universités françaises}

La thématique de l'évaluation - individuelle et collective - apparaît dans le paysage universitaire à partir du début des années 1980 avec les contributions de Laurent Schwartz et de Pierre Bourdieu, qui soulignent conjointement les faiblesses du contrôle et des incitations présentes au sein des universités françaises. Les syndicats, en acceptant, en 1988, la mise en place de primes différenciées pour les collègues, ouvrent aussi un peu plus la voie à l'individualisation des rémunérations, prémices à une individualisation des services sur la base d'une évaluation. Toutefois, d'une façon générale, le gouvernement n'aborde pas de front la question de l'évaluation individuelle des enseignants-chercheurs durant cette période. La priorité porte plutôt sur la coordination de la recherche scientifique au niveau national, notamment dans les domaines techniques, et l'évaluation est réalisée principalement lors d'appels à projets et se concentre sur les équipes.

L'évolution des perspectives autour de l'évaluation se réalise conjointement avec la mise en place progressive de la contractualisation entre l'État et les établissements d'enseignement supérieur, à partir de 1989, sous l'égide de Lionel Jospin. L'idée que l'évaluation doit permettre de récompenser les structures vertueuses, au détriment de celles qui le sont moins, prend peu à peu de l'importance dans l'environnement universitaire. En 1984 est ainsi créé le Comité national d'évaluation [https://www. cne-evaluation.fr/], ancêtre de l'AERES et de l'HCERES, qui a pour mission d'évaluer l'ensemble des établissements publics à caractère scientifique, culturel et professionnel : universités, écoles et grands établissements relevant de la tutelle du ministre chargé de l'enseignement supérieur. L'évaluation s'installe alors rapidement dans le paysage de l'enseignement supérieur, mais ne semble pour le moment ne concerner que les structures ${ }^{4}$. Le débat se déplace alors très vite au cours des années 1990 sur la question des indicateurs adaptés à l'évaluation des équipes ${ }^{5}$.

La production et l'usage des indicateurs se fait alors à un niveau de plus en plus fin, des laboratoires aux individus. La création de l'AERES par la loi de programme pour la recherche de 2006, qui englobe le CNE, aboutit à la notion de " publiant » comme critère d'évaluation des équipes de chercheurs, consacrant ainsi le glissement d'une évaluation collective à une évaluation individuelle des collègues. Un glissement identique a lieu aussi au CNRS. À cette période, des classements de revues émergent et resteront, pour de nombreuses années, la référence pour évaluer la recherche individuelle et collective. Ainsi, au début des années 2000, l'évaluation individuelle rentre progressivement dans les mœurs au sein des laboratoires, avec l'idée qu'une saine émulation au sein des équipes bénéficiera à la collectivité tout entière.

4 « La nouveauté radicale avec la loi de 1984 et l'installation du CNE en 1985 était que l'on passait des discours sur l'évaluation à la mise en place de l'évaluation elle-même. Ce qui déplaçait le débat de la discussion de principe (faut-il une évaluation ou pas ?) à une discussion de méthode (comment évaluer et avec quelles conséquences ?). On ne peut d'ailleurs qu'être surpris de la rapidité avec laquelle l'évaluation, dont peu de gens parlaient avant 1980, est devenue à la fin des années 1990 un thème assez consensuel, dans son principe au moins » (Mérindol, 2008).

5 Cela conduit notamment à la création de l'Observatoire des sciences et techniques. La question de l'évaluation correcte des différentes missions dévolues aux établissement est ainsi posée. 
Conjointement les rapports d'Éric Espéret (2001) et Bernard Belloc (2003) sont particulièrement virulents à l'égard des enseignants-chercheurs, dont ils préconisent de mieux contrôler l'activité - notamment en recherche - tout en ouvrant la voie à la redéfinition de leurs services. Ils reprennent l'idée d'une contractualisation entre l'enseignant-chercheur et son université, pouvant déboucher sur une modulation de services ${ }^{6}$. L'intégration des activités "d'animation et de responsabilité collective » aux missions des enseignants-chercheurs sera aussi repris dans le projet présenté par Valérie Pécresse, alors même que ceux-ci contribuent à déprofessionnaliser le métier d'enseignant-chercheur et interrogent, en retour, sur les critères d'évaluation à retenir face à l'éclatement des missions (La Broise, 2013).

La période est marquée par des changements institutionnels profonds, qui sont vécus comme une injonction pour l'université française à accroître sa compétitivité au sein d'un environnement de plus en plus mondialisé et concurrentiel (processus de Bologne, classement du Times et de Shanghai, etc.). Parallèlement, l'université a continué d'évoluer. La massification de l'enseignement supérieur se poursuit et l'université diversifie ses formats d'enseignement, qui correspondent de plus en plus difficilement au cadre statutaire des $192 \mathrm{~h}$ équivalent TD (formation continue, formation professionnelle, LMD, etc.). Enfin, l'information sur la production des universitaires, notamment scientifique, devient de plus en plus aisément disponible en ligne, facilitant les évaluations et exacerbant les comparaisons entre les individus et entre les équipes ${ }^{7}$. Le développement des indices de citation, et celui des classements de revues, conduiront au sein de l'espace européen à une véritable " fièvre de l'évaluation » (Gingras, 2008), dont les universitaires sont parfois eux-mêmes les promoteurs, et qui sera exacerbée par les réformes entreprises au cours de cette période autour de l'université.

En France, la loi LRU du 10 août 2007, qui vise officiellement à réhabiliter l'université et la recherche française en rendant les établissements autonomes, a suscité peu d'opposition de la part de la communauté universitaire (Collard, 2018). Comme le rappelle Collard (2018), il est vrai que la notion d'autonomie ne constituait plus vraiment une rupture institutionnelle puisqu'elle était présente dans l'environnement universitaire au moins depuis la loi Faure. A contrario, le projet de réforme des statuts des enseignants-chercheurs de 2009, qui s'inscrivait pourtant dans la droite ligne de la LRU et s'inspirait du rapport de Bertrand Schwartz (2008), a suscité rapidement l'inquiétude, puis la mobilisation de la communauté universitaire. Le projet de décret prévoyait tout d'abord le principe d'une évaluation systématique et régulière de l'enseignant-chercheur, appelé à fournir tous les quatre ans un rapport d'activité qui, après avoir été visé par le président de l'université, était transmis au CNU. Le décret ne précisait toutefois ni la finalité, ni la postérité de cette évaluation. Le projet de décret prévoyait ensuite le principe d'une modulation de service à la discrétion du président de l'université.

La juxtaposition des deux mesures au sein d'universités autonomes, a fait craindre aux collègues une modulation à la hausse de leurs services d'enseignement pour répondre aux besoins d'universités financièrement exsangues. Le mouvement

6 Ces dispositions seront combattues par les syndicats autonomes, FO et le SNESUP, et soutenues par la CPU et le SGEN-CFDT.

7 Les $h$ index se calculent aisément à partir de Google Scholar. 
au sein de la communauté universitaire a abouti finalement à la réintroduction de l'accord des intéressés afin de mettre en place une modulation de service, comme le prévoyait la première version du décret de 1984, mais contre l'avis de la CPU cette fois-ci! Le CNU voyait aussi son rôle confirmé comme principale instance d'évaluation des universitaires, aboutissant, comme le souligne Mérindol (2010), à « un compromis surprenant, renforçant à la fois l'autonomie des établissements et le rôle national du CNU ».

La défiance s'était pourtant durablement installée entre la tutelle et les universitaires, toujours inquiets des perspectives ouvertes par l'évaluation individuelle dans le cadre du rapport d'activité quadriennal. Le mouvement de 2009, qui a été porté par les enseignants-chercheurs, a instauré un profond malaise au sein de la communauté universitaire autour de la question de l'évaluation individuelle, systématique et récurrente. Comme le souligne Mérindol (2010), la violence des réactions provenait sans doute de la découverte, par une grande partie des universitaires, d'un important et lent déplacement, depuis les années 1980, d'un système central, piloté par l'État, vers un système décentralisé, s'appuyant sur des établissements de plus en plus autonomes. L'autonomie des établissements découle ainsi naturellement de la mise en place d'un système d'évaluation individuel des $\mathrm{RH}$, considéré comme un outil de « pilotage » de l'organisation.

II n'en reste pas moins que les enseignants-chercheurs ont anticipé en 2009 une modification profonde de leur métier, avec des enjeux autant identitaires que statutaires (Descombes, 2009 ; Collard, 2018). Une telle évaluation individuelle ne sera toutefois jamais vraiment mise en place. En 2012, la tutelle annonce un moratoire sur l'évaluation individuelle des enseignants-chercheurs par le CNU et la mise en œuvre d'une réflexion autour d'un nouveau dispositif. Cette réflexion aboutira en 2014 au suivi de carrière tel que nous le connaissons aujourd'hui, qui cristallise actuellement les oppositions entre politiques et universitaires autour de l'évaluation individuelle et récurrente des enseignants-chercheurs.

\section{La mise en œuvre de l'évaluation individuelle des enseignants-chercheurs : du suivi de carrière au dossier unique}

Un projet de révision du décret initial, proposé le 2 décembre 2013, aboutit au décret n²014-997 du 2 septembre 2014. Deux modifications mineures sont apportées au projet de 2009. Le terme " évaluation " est pudiquement remplacé par celui de " suivi de carrière ", et la périodicité de l'évaluation passe de quatre à cinq ans ${ }^{8}$.

\section{La mise en œuvre du suivi de carrière}

D'un point de vue pratique, le suivi de carrière est confié au CNU. II prend appui sur un rapport d'activité établi par l'enseignant-chercheur, qui mentionne l'ensemble

8 Pour les collègues nommés depuis au moins cinq ans, qui partent à la retraite dans plus de quatre ans, et qui n'ont pas bénéficié d'un avancement de grade au cours des cinq dernières années. Les collègues peuvent aussi soumettre volontairement un dossier au titre du suivi de carrière. 
de ses activités (activités pédagogiques, activités de recherche, tâches d'intérêt général), et retrace leurs évolutions éventuelles. Des souhaits et des besoins peuvent également y être exprimés (Bulletin officiel du 14 mai 2015). L'enseignant-chercheur envoie son rapport d'activité au président ou directeur de l'établissement auquel il est rattaché, et le rapport est ensuite soumis au Conseil académique, qui émet un avis sur les activités pédagogiques et les tâches d'intérêt général y figurant. Le rapport est ensuite transmis à la section du CNU dont relève l'intéressé, accompagné de l'avis du Conseil académique (ou de l'organe compétent) et des précisions que l'enseignant-chercheur a souhaité formuler. II est possible de joindre au dossier le rapport HCERES de l'unité pour une meilleure compréhension du contexte professionnel dans lequel évolue le collègue concerné. Les recommandations de la section doivent ensuite être adressées à l'enseignant-chercheur et au président ou directeur de l'établissement. Le Bulletin Officiel précise que ces recommandations

sont prises en compte par ce dernier en matière d'accompagnement professionnel des personnels. Elles constituent par là même un outil RH favorisant le développement des potentiels et l'épanouissement professionnel.

Après plusieurs années d'expérimentation, la CPU, la CP-CNU et la DGRH du Ministère de l'enseignement supérieur, de la recherche et de l'innovation s'accordent, en 2016, pour généraliser le suivi de carrière à partir de la campagne de 2018 à l'ensemble des établissements universitaires, chaque établissement voyant une partie de son corps enseignant convoqué chaque année à ce titre, en fonction de la section $\mathrm{CNU}$ de rattachement. Au terme des cinq ans, tous les universitaires d'un même établissement auraient dû être confrontés à une telle procédure. La participation au suivi carrière n'a pourtant cessé de décroître depuis 2018, comme l'indique la figure 1 . Ainsi, le taux de participation des enseignants-chercheurs " prioritaires » était de 25,5 \% en 2018, 22,5\% en 2019 et 17,7 \% en 2020. Cette dernière année, 955 universitaires " prioritaires " ont déposé un dossier sur les 5401 recensés. En outre, 191 universitaires " non prioritaires " ont déposé un dossier. Au total, 1146 enseignants-chercheurs ont donc participé au dispositif (DGRH, 2021b).

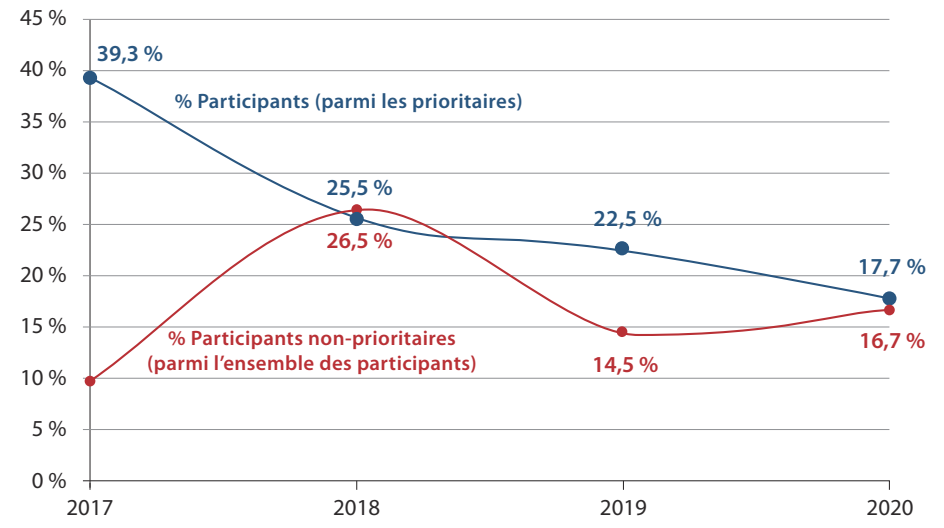

Figure 1 : Taux de participation des enseignants-chercheurs " prioritaires » au suivi de carrière de 2017 à 2020. Source : DGRH, ministère de l'Enseignement supérieur, de la Recherche et de I'Innovation 
La baisse continue du taux de participation au suivi de carrière s'explique en grande partie par la réticence de certaines sections CNU vis-à-vis de son application, comme l'indique les données de la figure 2. Ladite baisse s'observe aussi dans les sections qui sont favorables au suivi de carrière. En outre, il semble exister un manque de motivation de la part de certains établissements pour le mettre en application (DGRH, 2021b). Au final, $55 \%$ des dossiers ont reçu un avis pour l'établissement et pour les enseignants chercheurs, et $15 \%$ des dossiers n'ont reçu qu'un avis pour l'enseignant concerné. Enfin, $30 \%$ des dossiers soumis n'ont pas été examinés par le CNU (DGRH, 2021a).

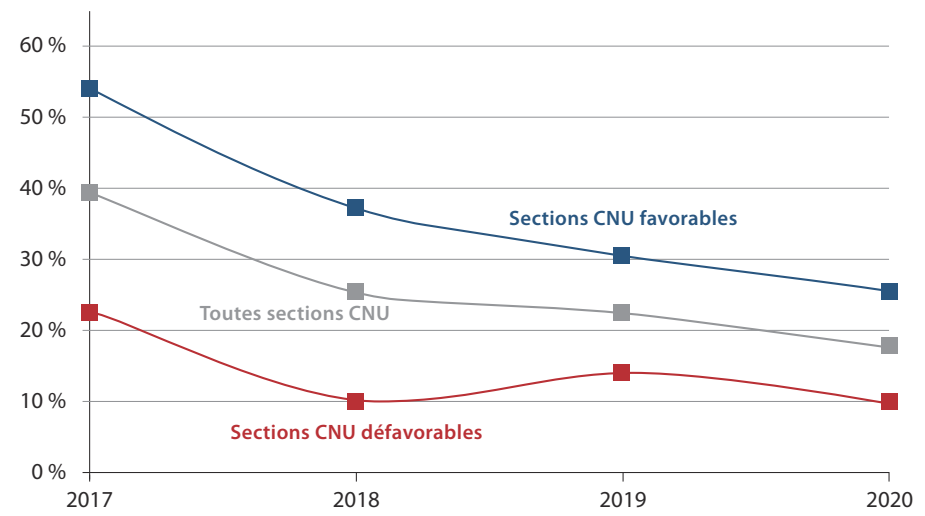

Figure 2 : Évolution des sections du CNU favorables ou non au suivi de carrière Source: DGRH, ministère de l'Enseignement supérieur, de la Recherche et de l'Innovation

Si le ministère et la CPU se sont évertués à rassurer la communauté universitaire quant à l'impossibilité d'utiliser à terme le suivi de carrière pour moduler les services des enseignants chercheurs, comme l'indiquent les courriers du 2 et 6 juin 2016 envoyés respectivement par le ministère et par la CPU, plusieurs syndicats ont dénoncé les intentions cachées d'un tel dispositif dans un contexte de restrictions budgétaires (encadré 1). La CP-CNU, qui avait, dans un premier temps, approuvé le dispositif dans une perspective d'aide à des collègues en difficulté, est ensuite devenue beaucoup plus critique compte tenu du peu de retour effectué par les établissements. Au sein du CNU, les sections sont partagées. En 2020, 31 sections (sur 57) se sont déclarées défavorables au dispositif ou n'ont pas rendu d'avis de suivi de carrière aux enseignants-chercheurs ayant déposé un dossier, et 15 d'entre elles ont publié des motions contre le suivi de carrière (DGRH, 2021b). Les sections défavorables comprennent l'intégralité des sections droit-économie-gestion, ainsi que la grande majorité des sections de lettres et sciences humaines, plus quelques sections dans le domaine des sciences et techniques. Les avantages et les inconvénients de suivi de carrière sont présentés dans le tableau 1. 
Tableau 1 : Suivi de carrière : avantages et inconvénients

\begin{tabular}{|c|c|}
\hline Avantages & Inconvénients \\
\hline $\begin{array}{l}\text { - Conseil aux collègues concernant le } \\
\text { déroulement de leur carrière } \\
\text { - Détection des collègues en grande difficulté } \\
\text { - Proposition d'un accompagnement, d'une aide } \\
\text { face à des problèmes particuliers } \\
\text { - Soupape d'expression pour les collègues (le } \\
\text { CNU comme seule instance indépendante) } \\
\text { - Formulation de recommandations aux } \\
\text { établissements dans l'intérêt des collègues } \\
\text { - Photographie de la population de la section }\end{array}$ & $\begin{array}{l}\text { - Imprécisions quant au cadrage et aux objectifs } \\
\text { - Craintes sur la finalité de l'usage du suivi de } \\
\text { carrières par les établissements (modulation } \\
\text { des services, retombées locales) } \\
\text { - Insuffisance des moyens alloués au CNU } \\
\text { - Redondance avec les évaluations existantes } \\
\text { (promotions, PEDR, CRCT, etc.) } \\
\text { - Inutilité compte tenu du peu de retour par les } \\
\text { établissements. } \\
\text { - Opposition au caractère obligatoire de } \\
\text { l'évaluation }\end{array}$ \\
\hline
\end{tabular}

Source: élaboration des auteurs d'après des documents CP-CNU

D'une manière générale, la plupart des sections favorables au suivi de carrière le considèrent comme un accompagnement de l'enseignant-chercheur au travers d'un regard extérieur porté par les pairs du CNU sur l'ensemble de ses activités. II doit demeurer bienveillant et bénéficier prioritairement à l'intéressé. Le CNU se transforme alors en cellule d'écoute, pouvant éventuellement détecter et signaler des collègues en souffrance. Certaines sections distinguent le retour aux établissements du retour aux collègues. L'incertitude sur les objectifs du suivi de carrière, et le peu de visibilité sur l'utilisation en étant faite par les établissements, sont souvent mis en avant par les sections du CNU qui n'appliquent pas le suivi de carrière. Le suivi de carrière, dans son acception bienveillante, serait amené à disparaître pour être remplacé par une évaluation moins bienveillante, voire une classification autoritaire des collègues actuellement en poste. Le caractère obligatoire du dispositif, l'absence de moyens supplémentaires et la dépense de fonds publics découlant du suivi de carrière sont aussi régulièrement dénoncés par les sections qui refusent de l'appliquer.

\section{Quel avenir pour l'évaluation individuelle et systématique des enseignants-chercheurs?}

Comme le remarquent avec justesse Côme et Rouet (2016)
la question du comment évaluer les intervenants est une question récurrente des politiques de ressources humaines, plus particulièrement dans le cadre du manage- ment des services (Chanut et Rojot, 2011). Elle est toutefois inséparable de la question du pourquoi (...). Les notions de transparence, de justesse, d'équité et d'acceptabi- lité du système d'évaluation deviennent des conditions nécessaires de réussite et de pérennité du système.

Dans cette perspective, l'erreur stratégique commise par la tutelle a été, en 2009, de lier l'évaluation individuelle des enseignants-chercheurs à la modulation de leurs services (et implicitement, au respect de l'équilibre budgétaire des établissements). Un tel objectif de gouvernance, qui était le même qu'en 1984, n'a pas été perçu comme juste par la plupart des collègues fortement investis dans leurs différentes missions au sein de l'université, et qui ont vu tendanciellement leur pouvoir d'achat diminuer au gré du blocage du point d'indice (figure 3). 


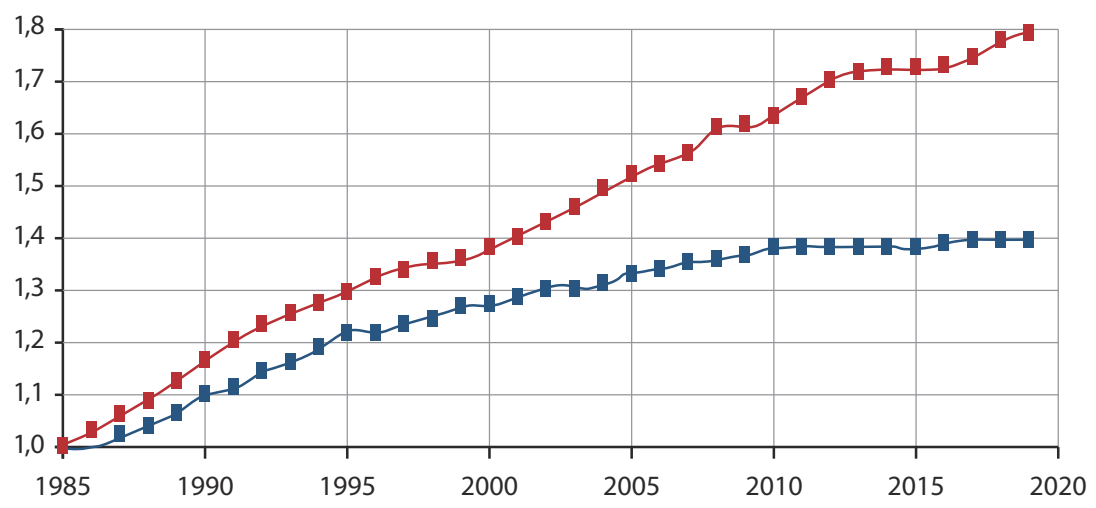

Figure 3 : Évolution des prix en rouge (inflation cumulée) et de la valeur du point d'indice brut en bleu Note : Valeur 1 en 1985 selon l'INSEE (pour les prix) et feuille de paie (pour l'indice). Source : d'après un document SNESUP

Un climat de défiance s'est alors durablement installé entre la tutelle et les universitaires. Le redéploiement de l'évaluation individuelle " au service des enseignants-chercheurs " n’a pas été jugé assez crédible pour emporter l'adhésion. La situation de crise de l'enseignement supérieur, confronté à la croissance continue des effectifs étudiants, engendre une diminution désormais tendancielle des enseignants-chercheurs en poste (figure 4). Doit-on dès lors être surpris par le fait que le rapport 73 de l'IGAENR, publié en 2015, recommande de supprimer la nécessité de l'accord de l'intéressé afin de moduler les services des enseignants-chercheurs (dans une fourchette identique à celle prévue en 1984) ?

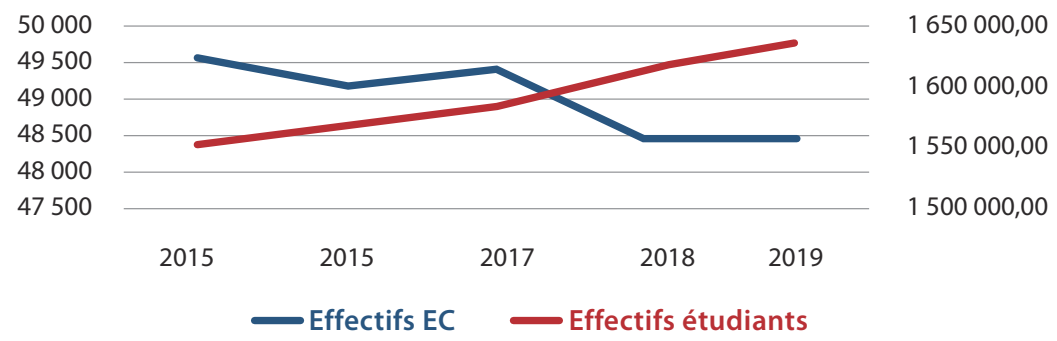

Figure 4 : Évolution des effectifs d'enseignants-chercheurs et d'étudiants Source : d'après un document CP-CNU

Les enjeux diffèrent selon les sections, et la diminution de la pression estudiantine dans certaines disciplines explique, peut-être, des prises de position parfois plus conciliantes. La communauté des enseignants-chercheurs en sciences de gestion et du management a réaffirmé régulièrement son opposition au suivi de carrière dans différentes motions. Les gestionnaires ne sont pas fermés à la logique même de l'évaluation individuelle; la culture de l'évaluation est inhérente à la gestion, celle-ci étant déjà à l'œuvre via des rankings internationaux ou des certifications d'établissements, par exemple. Au demeurant, ce sont des théoriciens de notre discipline qui sont à l'origine des concepts sous-jacents aux pratiques managériales, mais actuellement dévoyés par la tutelle. 
La forte demande sociale pour les formations en sciences de gestion et du management, les exigences de la professionnalisation et le sous-effectif chronique du corps enseignant en section 06 conduisent à des services souvent très lourds, qui induisent un fort volant d'heures complémentaires. Les universités peinant à financer leurs activités, la modulation des services d'enseignement pourrait apparaître comme une source d'économie non négligeables pour l'État et pour les universités. Par ailleurs, en l'absence de mouvement compensatoire inverse, ceci viendrait à diminuer un peu plus l'attractivité sur le marché de l'enseignement supérieur de l'université française, par rapport aux universités étrangères et aux business schools.

Tous les acteurs campant sur leurs positions respectives, le ministère tente actuellement de reprendre la main sur cette question en s'appuyant sur les conclusions du rapport de Fabienne Blaise, Pierre Desbiolles et Patrick Gilly, remis en avril 2021, qui devait initialement porter uniquement sur le recrutement des enseignants-chercheurs. Les auteurs du rapport adoptent le suivi de carrière comme un « fil rouge " au prétexte que l'article de la LPR introduisant suppression et dérogation à la qualification toucherait « à tous les moments-clés de la vie professionnelle »! Afin " d'assurer à tout enseignant-chercheur un accompagnement régulier ", Fabienne Blaise, Pierre Desbiolles et Patrick Gilly suggèrent notamment que les établissements « se saisissent du suivi de carrière de leurs enseignants-chercheurs, en s'appuyant sur le $\mathrm{CNU}$ " ${ }^{9}$. Ils proposent d'instaurer un " dossier unique " qui serait numérisé et suivrait chaque enseignant-chercheur tout au long de sa carrière, "favorisant le partage d'informations entre enseignant-chercheur, CNU et établissement, l'instruction des demandes de promotion et la prise de décision ". Bien entendu, ce dossier unique, qui ressemble à s'y méprendre un livret d'évaluation fait sur mesure pour les établissements, pose de nombreuses questions qu'il conviendra d'étudier avec la plus grande attention si le dispositif se mettait en place.

\section{Conclusion}

L'évaluation individuelle et systématique des enseignants-chercheurs est une question complexe. Des processus se sont progressivement mis en place pour répondre à l'autonomisation progressive des établissements de l'enseignement supérieur entre 1968 et 2007. Toutefois, la communauté universitaire a toujours été extrêmement prudente à ce niveau, comprenant que l'évaluation était potentiellement porteuse d'une redéfinition des conditions de travail des enseignants-chercheurs, dans un contexte assumé d'austérité budgétaire pour les établissements. Si le projet de décret de 2009 a conduit à une crispation de la communauté universitaire, les dernières propositions du rapport Fabienne Blaise, Pierre Desbiolles et Patrick Gilly s'inscrivent dans la droite ligne de la LPR et semblent en passe de modifier sensiblement les procédures du suivi de carrière des enseignants-chercheurs. Nous pouvons souhaiter que la communauté d'enseignants-chercheurs en sciences de gestion et du management, comprenant nombre de spécialistes du contrôle organisationnel, apporte sa pierre au débat qui s'annonce autour de cette question.

9 Le renversement des rôles entre les établissements et le CNU s'inscrit dans un mouvement plus général qui concerne à la fois les promotions et le recrutement (RIPEC et repyramidage). 

Transmettre 



\section{L'enseignant-chercheur en sciences de gestion et du management à l'Université (Ré)concilier production et diffusion de la connaissance}

Marc Bollecker, Kirsten Burkhardt et Evelyne Poincelot

Le décret de 1984, modifié en 2009, fixe les dispositions statutaires communes applicables aux enseignants-chercheurs, particulièrement aux professeurs des universités et aux maîtres de conférences. Au-delà de la participation à la vie collective des établissements, leurs activités s'articulent autour de missions interconnectées de production et de diffusion de la connaissance scientifique. Les enseignants-chercheurs " participent à l'élaboration, par leur recherche, et assurent la transmission, par leur enseignement, des connaissances au titre de la formation initiale »; assurent « le développement, l'expertise et la coordination de la recherche fondamentale, appliquée, pédagogique ou technologique ainsi que la valorisation de ses résultats "; " participent au développement scientifique et technologique en liaison avec les grands organismes de recherche et avec les secteurs sociaux et économiques concernés; " contribuent au dialogue entre sciences et sociétés, notamment par la diffusion de la culture et de l'information scientifique et technique " (source : [https://www.legifrance.gouv.fr]). Dans l'accomplissement de ces missions, ils

jouissent d'une pleine indépendance et d'une entière liberté d'expression dans l'exercice de leurs fonctions d'enseignement et de leurs activités de recherche, sous les réserves que leur imposent, conformément aux traditions universitaires et aux dispositions du présent code, les principes de tolérance et d'objectivité (article L9852-2 du code de l'éducation).

En d'autres termes, les enseignants-chercheurs jouissent donc d'une latitude décisionnelle tout en étant soumis à des mécanismes qui les gouvernent. En les recrutant, le système leur délègue le pouvoir d'exercer leurs fonctions tout en posant les règles du jeu au sein desquelles ils se doivent de les assurer. Ce système institutionnel, composé de différents mécanismes, peut orienter ou contraindre leur liberté d'action. 
L'objet du chapitre est de montrer, dans une première partie, que les missions de production et de diffusion de la connaissance des enseignants-chercheurs en sciences de gestion et du management (notés ECG par la suite) s'adressent à un public particulièrement vaste. Pour autant, leur liberté d'action et les dilemmes auxquels ils sont confrontés conduisent à une diversité de profils / identités et au risque de devoir hiérarchiser ces deux missions. Dans une seconde partie, nous montrerons que les mécanismes du système universitaire et les injonctions demandées aux universités ou aux organisations gravitant autour de notre discipline contribuent à accentuer un tel risque. En conclusion, nous montrerons l'importance du CNU et nous explorons des voies complémentaires qui permettraient aux ECG de (re)concilier production et diffusion de la connaissance.

\section{Produire et diffuser des connaissances scientifiques en sciences de gestion et du management : des représentations différenciées}

Les ECG ont l'opportunité d'essaimer leurs connaissances auprès d'un vaste public d'étudiants : 135581 étudiants étaient inscrits en 2017-2018 dans une formation en gestion dans les universités publiques françaises, soit 7,7 \% des effectifs totaux de ces établissements (quasi identiques à la médecine, les effectifs en gestion se situent parmi les trois disciplines [sur 55] qui comptent le plus d'étudiants) (tableau 1). Le potentiel de diffusion des connaissances de la recherche est donc vaste en touchant un public qui, à son tour, peut irriguer la société par les stages, l'alternance et l'insertion professionnelle ou encore par la formation continue. Pour cette dernière, la gestion était, en 2013, la discipline ayant dispensé le plus d'heures (11 millions d'heures de cours, soit $21 \%$ du total des heures dispensées dans les universités françaises), à plus de 34400 stagiaires (selon les données les plus récentes disponibles sur [https://www.enseignementsup-recherche.fr]). Elle permet ainsi de répondre à une demande sociale très importante et une présence auprès des entreprises (Pras, 2010).

Tableau 1 : Effectifs enseignants et étudiants en 2017-2018 dans les universités françaises

\begin{tabular}{|c|c|c|c|c|c|c|c|c|c|}
\hline \multirow[b]{2}{*}{$\begin{array}{l}\text { Sciences } \\
\text { juridiques }\end{array}$} & \multirow{2}{*}{$\begin{array}{c}\begin{array}{c}\text { Effectifs } \\
\text { EC }\end{array} \\
3372\end{array}$} & \multicolumn{2}{|c|}{$\begin{array}{c}\text { Effectifs } \\
\text { professeurs } \\
\text { des universités } \\
\text { et assimilés }\end{array}$} & \multicolumn{2}{|c|}{$\begin{array}{c}\text { Effectifs maîtres } \\
\text { de conférences } \\
\text { et assimilés }\end{array}$} & \multirow{2}{*}{$\begin{array}{c}\begin{array}{c}\text { Effectifs } \\
\text { second } \\
\text { degré }\end{array} \\
2\end{array}$} & \multirow{2}{*}{$\begin{array}{c}\begin{array}{c}\text { Total } \\
\text { enseignants }\end{array} \\
3374\end{array}$} & \multirow{2}{*}{$\begin{array}{c}\text { Effectifs } \\
\text { étudiants }\end{array}$} & \multirow{2}{*}{$\begin{array}{c}\begin{array}{c}\text { Ratio } \\
\text { étudiants } \\
\text { EC }\end{array} \\
55\end{array}$} \\
\hline & & 2180 & $65 \%$ & 1192 & $35 \%$ & & & & \\
\hline Médecine & 5589 & 4158 & $74 \%$ & 1431 & $26 \%$ & 0 & 5589 & 136627 & 24 \\
\hline $\begin{array}{l}\text { Sciences } \\
\text { de gestion }\end{array}$ & 2111 & 484 & $23 \%$ & 1627 & $77 \%$ & 1704 & 3815 & 135581 & 64 \\
\hline $\begin{array}{l}\text { Sciences de } \\
\text { l'éducation }\end{array}$ & 677 & 173 & $26 \%$ & 504 & $74 \%$ & 37 & 714 & 87606 & 129 \\
\hline Psychologie & 741 & 197 & $27 \%$ & 544 & $73 \%$ & 0 & 741 & 63709 & 86 \\
\hline $\begin{array}{l}\text { Sciences } \\
\text { écono- } \\
\text { miques }\end{array}$ & 1732 & 547 & $32 \%$ & 1185 & $68 \%$ & 3 & 1735 & 37036 & 21 \\
\hline National & 55680 & 20037 & $36 \%$ & 35643 & $64 \%$ & 12867 & 68547 & 1755708 & 32 \\
\hline
\end{tabular}

Source : [https://data.enseignementsup-recherche.gouv.fr] 
Le poids conséquent de la gestion mérite cependant d'être discuté au regard des effectifs d'enseignants-chercheurs. En comparaison aux disciplines accueillant le plus d'étudiants dans l'enseignement supérieur public français, la gestion se caractérise par le plus faible taux d'encadrement, aussi bien des étudiants (64 par ECG en 2017-2018, contre 24 en médecine et 55 en sciences juridiques), que des professeurs des universités ( $23 \%$ du total des ECG) (tableau 1). Sans vouloir sous-estimer ni douter de la capacité des universitaires en gestion à encadrer un volume important d'étudiants tout en assurant en parallèle une activité de production de la connaissance (et d'autres activités), sans même vouloir contester l'importante production scientifique réalisée par le biais des revues, congrès, conférences et médias, et l'implication dans les sociétés savantes, nous pouvons cependant raisonnablement nous demander si le temps consacré à la recherche - ou à d'autres activités - peut être équivalent à celui de chercheurs de disciplines mieux encadrées. Si les activités d'enseignement représentent en moyenne $56,4 \%$ du temps total de l'activité annuelle d'un enseignant-chercheur en sciences humaines et sociales et en sciences et technologies (la recherche $33,3 \%$, les activités d'administration et de pilotage $5,1 \%$ et les réponses aux courriels 5,2 \%) (Ducrocq et Gervais, 2013), I'hypothèse selon laquelle les disparités entre les disciplines soient fortes n'est pas à écarter.

Certes, le temps passé sur une activité ne permet pas d'en cerner son efficacité, d'autant plus que les formations en gestion sont soutenues par de nombreux enseignants du secondaire et par des vacataires qui viennent compenser partiellement ce déficit. L'enseignement de la gestion peut se pratiquer selon un modèle direct (praticiens qui transfèrent leurs pratiques), un modèle pédagogique (observation et diffusion des pratiques par des enseignants professionnels), un modèle académique (développer les savoirs sur les pratiques à partir de démarches scientifiques) (Laroche, 2007). Cependant, un tel soutien ne peut soustraire les ECG de leur mission de direction, de conseil, de tutorat, d'orientation des étudiants, de leur insertion professionnelle, de participation aux jurys d'examen et de concours et à la vie collective des établissements (conformément à l'article 3 du décret de 1984 modifié en 2009).

La diversité des missions confiées statutairement aux ECG, et la masse d'étudiants présents dans les filières de gestion (ainsi que d'autres facteurs, comme indiqué ci-dessous), invitent à des représentations différentes de leur métier, et notamment de l'articulation entre production et diffusion de la connaissance. Par exemple, concernant leur responsabilité, Royer (2011) rappelle le débat existant

entre les partisans d'une recherche engagée dans la société où le chercheur doit répondre des conséquences de ses travaux, d'une part et ceux qui limitent la responsabilité du chercheur à la production de connaissances et donc le considèrent non responsable des utilisations négatives qui peuvent être faites de ses connaissances, d'autre part.

Le lien entre la production et la diffusion des connaissances dans les activités de l'universitaire en gestion renvoie à la question souvent débattue et controversée de leur impact sociétal : « des voix s'élèvent du monde de l'entreprise pour réclamer de la recherche en gestion davantage de pertinence et de retombées pratiques " (Gillet et Zimnovitch, 2011). Cependant, si les applications des travaux peuvent " avoir des répercussions importantes aussi diverses que la structure des industries ou les 
revenus, le bien-être et la santé des salariés et consommateurs " (Royer, 2011), l'impact en reste difficilement mesurable : les effets des enseignements sont dilués et étalés (Laroche, 2007), notamment en raison d'un temps de latence entre la fin de la formation et le moment où le diplômé peut influer sur les pratiques. Par ailleurs, l'inertie organisationnelle et le rôle des consultants ou des médias dans les transformations des pratiques peuvent en limiter l'impact.

L'articulation de la production de la connaissance en sciences de gestion et du management et de sa diffusion par les enseignants-chercheurs n'est donc ni évidente ni homogène. Courpasson et Guedri (2007) mettent ainsi en lumière une typologie de trois ethos différents chez les chercheurs en école et en université, construite sur " la base du rapport entre science et entreprise, c'est-à-dire sur le jugement d'utilité de la science »: (1) les académiques, quadragénaires titulaires d'une HDR, très actifs dans la vie scientifique, qui accordent une primauté à la valeur scientifique sur la valeur économique ; (2) les experts activistes, les quinquagénaires moins chercheurs sans HDR, exerçant plutôt en école, pouvant être hostiles au modèle académique et dont l'engagement entrepreneurial est une continuité de l'activité scientifique; et (3) les hybrides, chercheurs pédagogues et managers, jeunes maîtres de conférences confrontés à l'ambivalence des choix d'activités, et entrant en concurrence (carrière par la publication et possibilité d'agrégation vs. nécessité d'engagement local dans des responsabilités lourdes).

Laroche (2007), en reprenant les six modèles d'éducation au management de Korpiaho et al. (2007), autrement dit " traditionnel ", "MBA ", " scientifique ", " des compétences ", "fondés sur l'action », et " critique ", dévoile également cette hétérogénéité. Si une telle approche vise à explorer les relations incertaines qu'entretiennent les enseignements et les pratiques de gestion (diffusion), elle sous-tend aussi la question de leur articulation avec la production de connaissances par les ECG. Cette articulation n'apparaît explicitement que dans les modèles « scientifique » et " critique » dans lesquels la conception de l'enseignant serait d'incarner respectivement un chercheur :

- qui expose les étudiants à la qualité de théories robustes et à des faits avérés, les entraînant à la réflexion, la lecture, l'écriture afin de former des managers professionnels et responsables;

- engagé et facilitateur, qui apporte des ressources intellectuelles et des espaces pour une réflexion critique sur l'idéologie et la justice dans le management afin de former des professionnels et intellectuels politiquement lucides.

L'articulation de la production de la connaissance et de sa diffusion par l'ECG peut le confronter à un dilemme de nature épistémologique et didactique : il " peut être un relais des pratiques (...) traducteur et agent légitimateur de celles-ci. Mais il fonde sa légitimité sociale dans sa capacité à éclairer la société sur des problématiques complexes. Il se positionne dès lors en constructeur de théories " (Dupuy et al., 2006). Une telle articulation peut être liée à l'attitude que les ECG ont à l'égard du système de publication. Dans une comparaison franco-québécoise réalisée sur 107 universitaires, Lussier et Chanlat (2017) dévoilent huit profils différents vis-à-vis du système actuel de publication en gestion, selon qu'ils y participent ou non (tableau 2). Nous pouvons raisonnablement formuler l'hypothèse selon laquelle, si le lien entre production et diffusion de la connaissance semble plus facile à réaliser chez les convaincus 
et mobiles sans attache (1), voire chez les ambivalents et les résignés (3), il l'est moins chez les pédagogues et administrateurs (2), même s'ils ont une croyance forte au système, et certainement très faible pour les non-conformistes et les résistants (4).

Tableau 2 : Typologie des universitaires en gestion sur leur croyance au système de publication

\begin{tabular}{|c|c|c|}
\cline { 2 - 3 } \multicolumn{1}{c|}{} & $\begin{array}{c}\text { Croyants } \\
\text { (adhérant au système) }\end{array}$ & $\begin{array}{c}\text { Non-croyants } \\
\text { (n'adhérant pas au système) }\end{array}$ \\
\hline $\begin{array}{c}\text { Enseignants participant } \\
\text { au système }\end{array}$ & $\begin{array}{c}\text { Convaincus mobiles } \\
\text { sans attache (1) }\end{array}$ & Ambivalents résignés (3) \\
\hline $\begin{array}{c}\text { Enseignants ne participant pas } \\
\text { au système }\end{array}$ & Pédagogues administrateurs (2) & Non-conformistes résistants (4) \\
\hline
\end{tabular}

\section{Des mécanismes de gouvernance cloisonnant l'activité de recherche et d'enseignement}

Les ECG disposent d'une latitude décisionnelle qui forge différentes identités, comme cela a été développé dans la première partie. Mais ils sont également soumis à des mécanismes qui délimitent leur liberté d'action. Cette représentation fait écho à la gouvernance, par exemple des entreprises où les actionnaires ont délégué au dirigeant le soin de les gérer selon leurs intérêts. Rappelons que le système de gouvernance recouvre l'ensemble des mécanismes posant les règles du jeu, le système institutionnel et les mécanismes le constituant, qui encadrent les décisions d'une organisation et qui délimitent ainsi leur liberté d'action (Charreaux, 1997, 2018). De tels mécanismes peuvent être intentionnels ou spontanés, spécifiques ou non :

Est considéré comme spécifique, tout mécanisme propre à l'entreprise délimitant le pouvoir discrétionnaire des dirigeants dans le sens où son action influence exclusivement les décisions des dirigeants des entreprises (Charreaux, 1997).

La structure, les règles formelles sont des mécanismes spécifiques (assemblées générales des actionnaires, conseil d'administration, règlements internes, etc.). Sont considérés comme intentionnels les mécanismes qui ont été conçus pour délimiter l'action des dirigeants d'entreprises (environnement règlementaire et procédures formelles). Ils s'opposent aux mécanismes spontanés qui peuvent délimiter leur liberté d'action de façon occasionnelle alors que leur raison d'être n'est pas liée à la gouvernance d'entreprise (différents marchés, attentes implicites des parties prenantes, confiance, réputation, etc.). Par analogie avec les entreprises, et en adaptant cette grille de lecture aux établissements universitaires, nous pouvons identifier différents mécanismes qui posent les règles du jeu aux enseignants-chercheurs, consistant à combiner les activités d'enseignement et de recherche, et qui contribuent à les cloisonner.

\section{Les mécanismes non spécifiques}

Les mécanismes non spécifiques à une université sont des mécanismes intentionnels, comme les lois, les règles déontologiques ou des mécanismes spontanés tels que la mise en concurrence des établissements publics et privés sur le marché de 
l'offre de la formation, ou encore le marché des financements de la recherche et de l'enseignement. Nous commençons par présenter les textes de lois qui exercent une influence sur quasiment tous les autres mécanismes de gouvernance.

\section{Les mécanismes non spécifiques intentionnels}

Différents textes régissent le monde de l'enseignement et de la recherche. Nous en exposons quelques-uns qui nous semblent particulièrement importants pour nos propos. Rappelons tout d'abord les textes portant sur le statut et sur l'évaluation des enseignants-chercheurs. Le texte général sur le statut d'enseignant-chercheur, signé le 6 juin 1984 (décret 84-431), regroupe les deux corps de professeurs des universités et de maîtres de conférences :

Le décret statutaire du 6 juin 1984 (art. 7) introduit une innovation qui ne sera pratiquement pas mise en application. II s'agissait de moduler ces services dans une fourchette de 0,5 à 1,5 du service de base, en fonction du degré de participation aux autres missions, dont la recherche et des prises de responsabilité (p. 79). Elle est supprimée sous le gouvernement Chirac en 1988. Elle sera réintroduite en 2009 sous un contexte différent (Mérindol, 2010).

La séparation entre les missions d'enseignement et de recherche a ensuite été formalisée dans le décret du 23 avril 2009 qui a apporté une précision : le temps de travail est constitué, pour moitié, par une activité de recherche et pour l'autre moitié, par les services d'enseignement déterminés par rapport à une durée annuelle de référence égale à 192 h équivalent TD. Lorsqu'ils accomplissent des enseignements complémentaires au-delà de leur temps de travail, les enseignants-chercheurs perçoivent une rémunération complémentaire. Une prime pour la recherche est versée deux fois par an et ce, indépendamment de l'activité recherche effective. Comme nous le verrons ultérieurement, l'enseignant-chercheur peut ainsi être financièrement incité à effectuer davantage d'heures complémentaires d'enseignement, aux dépens de la recherche.

Depuis début 2021, en lien avec la nouvelle loi de programmation de la recherche initiée par Madame Frédérique Vidal, ministre de l'Enseignement supérieur, de la Recherche et de l'Innovation votée antérieurement, des mesures sur les carrières concernant la prime de recherche et d'enseignement supérieur (PRES) permettront un rattrapage visant à accroitre l'attractivité du métier. De façon concrète, pour 2021, la PRES, jusqu'ici d'environ 1260 euros pour toutes les catégories, passe à 2350 euros pour les maîtres de conférences, et à 1840 euros pour les professeurs des universités. Dans notre discipline où $23 \%$ des enseignants-chercheurs sont des professeurs des universités, comme indiqué précédemment, cela implique une réelle difficulté pour obtenir un changement de corps (celui de maître de conférences à professeur des universités), dont l'obtention dépend d'un dossier de recherche solide. Ainsi, la prime récemment votée liée à la recherche est décroissante avec un changement de corps particulièrement difficile à obtenir !

Les textes fondateurs de la formation post-bac, ou la réforme LMD, qui s'imposent au système universitaire, favorisent également une telle perception. La rédaction de ces textes revêt évidement un caractère intentionnel et général mais toutes les conséquences leur étant attachées ont aussi renforcé le cloisonnement pour notre 
discipline. Elles ont également des conséquences sur d'autres mécanismes, notamment spontanés, tels que la mise en concurrence de l'offre universitaire face à l'offre privée, ou encore sur les attentes des parties prenantes sur l'offre de la formation. Elles seront détaillées dans les prochaines sections.

Nous terminerons notre présentation non exhaustive des textes par la loi relative à l'enseignement supérieur et la recherche de 2013, qui donne notamment naissance au Haut conseil de l'évaluation de la recherche et de l'enseignement supérieur (HCERES). En tant qu'instance d'évaluation du monde universitaire, il porte de façon segmentée une appréciation, d'une part, sur la recherche, en expertisant les laboratoires, l'école doctorale, les regroupements de laboratoire (expertise des maisons des sciences de l'homme, par exemple, pour les sciences humaines et sociales, etc.), et, d'autre part, sur le volet enseignement avec une expertise au niveau des formations. Cette évaluation reflète l'organisation même des établissements - ou groupe d'établissements - séparant dans ses structures l'enseignement et la recherche avec d'un côté, les composantes, et de l'autre, les laboratoires, avec des efforts récents réalisés pour les concilier (collegium, pôle thématique, etc.), à l'échelle de plusieurs disciplines.

\section{Les mécanismes non spécifiques spontanés}

Les lois ne sont pas sans conséquences sur d'autres mécanismes de gouvernance. Par exemple, la réforme LMD étroitement liée à l'espace européen de l'enseignement supérieur a été un révélateur de la mise en concurrence de l'offre universitaire face à l'offre privée, sur des formations relativement spécialisées telles que la gestion. Si, dans le domaine de la gestion en France, les universités ont toujours été en concurrence avec les écoles de management privées, une telle concurrence s'est accrue avec la réforme. Effectivement, elle a permis d'harmoniser les diplômes proposés par les différents établissements. De ce fait, elle a obligé

I'Université à se positionner dans une offre globale de formation, incluant des écoles qui, jusqu'à présent, n'avaient pas la possibilité d'offrir des diplômes de grade de master (...) elle inscrit l'université dans une logique de résultat (...) (taux de réussite, taux de poursuite d'études, taux d'insertion professionnelle, etc.). Cette considération est particulièrement importante pour les sciences de gestion et du management où les étudiants sont très sensibles à de tels critères et n'hésitent pas à s'orienter vers des écoles de commerce, même de moindre importance, qui répondraient mieux à leur attente (Bensebaa et Vandangeon-Derumez, 2008).

Ce renforcement de la concurrence contribue à cloisonner l'enseignement et la recherche car l'amélioration de l'insertion professionnelle passe assez peu par la recherche. La réforme LMD n'est pas sans conséquence sur l'offre même de formation. Si le site Internet [https://www.enseignementsup-recherche.gouv.fr] définit le master comme diplôme professionnalisant ouvert sur la recherche, l'offre de formation, afin de favoriser l'insertion professionnelle des étudiants dans un environnement fortement concurrentiel, continue de proposer des masters orientés « monde professionnel " ou orientés "monde de la recherche ", même si des efforts sont entrepris pour initier les étudiants inscrits dans les masters de la première catégorie aux enjeux de la recherche en sciences de gestion et du management (projet d'initiation à la recherche, par exemple). 
Deux autres marchés méritent d'être brièvement présentés : le marché du financement de la recherche et de l'enseignement, et le marché de l'emploi des ECG. Le financement de l'enseignement et de la recherche en gestion n'atténue pas non plus le cloisonnement. Les financements internes à l'établissement ne suffisent pas pour financer ni l'enseignement ni la recherche en gestion. Des financements externes doivent donc être obtenus. Ceux-ci financent très rarement les deux volets. Enfin, le marché de l'emploi des ECG en France présente différentes particularités. Qu'il s'agisse des écoles universitaires publiques ou privées en management, ce qui importe, ce sont souvent les publications d'un certain rang, donc le dossier recherche. Si nous restons dans le monde universitaire, bien qu'il existe différentes voies d'accès aux postes de professeur des universités (Paché, 2021b), le dossier recherche importe fortement pour sa progression. Or, comme nous le verrons ultérieurement, celui-ci pousse l'enseignant-chercheur à faire des arbitrages entre l'enseignement et la recherche, qui ne jouent pas systématiquement en faveur de la recherche et qui favorisent le cloisonnement entre eux.

\section{Les mécanismes spécifiques}

Nous présentons maintenant les mécanismes de gouvernance spécifiques à l'université : les relations avec les principales parties prenantes (mécanismes " spontanés ") ; le système incitatif et d'évaluation de l'enseignant-chercheur universitaire (mécanisme intentionnel).

\section{Les mécanismes spécifiques spontanés}

Si nous considérons les étudiants et les professionnels qui les recrutent, l'importance qu'ils accordent à la recherche est sans rapport avec le temps qu'un ECG doit y consacrer dans l'exercice normal de sa mission. Les étudiants en gestion souhaitent avant tout être rapidement opérationnels sur le marché du travail et être rassurés quant à leur capacité à décrocher rapidement un stage ou un emploi. Cet objectif sera, en considérant le temps de la formation limité, davantage atteint par l'acquisition de techniques les rendant directement opérationnels, que par l'apprentissage d'un recul sur les pratiques, et du développement d'un esprit critique quant à l'utilisation d'un outil de gestion, par exemple. La formulation des offres de stage, ou liées à un premier emploi, le marché de l'emploi lui-même, le contexte sanitaire et l'environnement concurrentiel des formations universitaires en gestion les invitent aussi à avoir une visée à court terme. C'est parfois quelques années après être immergés dans l'entreprise que certains cours plus axés sur la réflexion autour des organisations et des systèmes, prennent davantage de sens. L'ECG pourrait même être mieux évalué par les étudiants quand ses cours correspondent à leurs attentes, ce qui ne l'incite pas à s'attarder massivement sur la partie recherche de son enseignement. Ce ressenti de la part des étudiants / professionnels peut accentuer la perception de cloisonnement pour les ECG. Mais, peut-être, ont-ils aussi une part de responsabilité auprès des " professionnels " en ne leur communiquant pas suffisamment l'intérêt de leurs recherches ou en ne vulgarisant pas assez leurs articles scientifiques. Le cloisonnement entre l'enseignement et la recherche se ressent également au niveau 
des associations (entre les associations de master, les associations scientifiques et les associations professionnelles où les liens sont plutôt périphériques).

\section{Les mécanismes spécifiques intentionnels}

Le système incitatif et d'évaluation d'un établissement dépend de son organisation. Si celle-ci est bien entendu spécifique aux universités, nous retrouvons des points communs, comme le fait de séparer l'enseignement et la recherche. Les universités sont aussi structurées en silos avec une présence systématique des ECG sur site pour la partie enseignement, mais pas pour la recherche. Dans le contexte qui a favorisé le regroupement d'établissements afin de peser davantage sur la scène internationale de la formation post-bac, il est fréquent de constater une délégation uniquement de la partie recherche ou de fonctions support dans le cadre de communauté d'universités et établissements (COMUE), l'enseignement restant à l'échelle des établissements. L'évaluation nationale par le HCERES, déjà mentionnée, évalue aussi séparément l'enseignement et la recherche.

Les mécanismes incitatifs et d'évaluation des enseignants-chercheurs sont, eux aussi, très variables selon les universités. Cependant, des points communs peuvent à nouveau être avancés, notamment en ce qui concerne la rémunération des tâches d'enseignement et de la recherche, ainsi que du temps consacré à ces activités. En ce qui concerne la rémunération d'un ECG, la partie recherche est limitée et une prime est perçue indépendamment des efforts entrepris en matière de recherche, alors que la composante liée à l'enseignement et le suivi de stages / alternances peuvent, selon les établissements, ne pas l'être. Par ailleurs, nous l'avons vu, cette prime de recherche est désormais décroissante avec le changement de corps. Ainsi, si nous étudions le couple "rémunération obtenue relativement au temps passé à accomplir ses missions enseignement / recherche ", un maître de conférences pourrait mettre dans la balance la rémunération obtenue à court terme, et de façon certaine, par les heures complémentaires d'enseignement, les suivis de stagiaires ou d'alternants, et celle obtenue à moyen / long terme en accentuant les efforts sur la recherche (avec l'espoir peut-être de devenir professeur des universités). Un tel arbitrage ne joue pas systématiquement en faveur de la recherche dans notre discipline, et renforce donc le cloisonnement.

Au-delà de la rémunération, le temps pouvant être consacré à la recherche n'est pas neutre également dans l'arbitrage entre les missions, celui-ci pouvant être limité s'il accomplit des charges administratives importantes liées au sous-encadrement très fréquent dans notre discipline. Ainsi, Courpasson et Guedri (2007) indiquent que « la recherche française en management pâtit d'un déficit d'organisation et de gestion incitative à faire de la recherche : le décalage entre les outils de mesure de performance et la pauvreté des mécanismes d'encouragement et d'incitation est immense. Par ailleurs, il est réducteur de penser incitation comme synonyme de finances. La question du temps disponible est sans doute encore plus centrale dans les préoccupations des enseignants-chercheurs (Courpasson et Guedri, 2007). Ajoutons que, dans notre discipline, les formateurs ne sont pas seulement des enseignants-chercheurs et nous pouvons retrouver un autre cloisonnement, celui entre les vacataires, PRAG (professeur agrégé de l'enseignement du seconde degré) et enseignant-chercheur dont les missions sont différentes. Le tableau 3 synthétise les mécanismes menant 
aux cloisonnements présentés. Parmi cet éventail, il est intéressant de constater que ces derniers sont plus fréquents à des échelons où chaque université détient une plus grande latitude décisionnelle, elle-même bornée par d'autres impératifs comme par exemple, améliorer l'insertion professionnelle des étudiants et de la place qu'elle souhaite / peut donner à notre discipline.

Tableau 3 : Mécanismes de gouvernance attachés à l'université : une représentation non exhaustive

\begin{tabular}{|c|c|c|}
\hline Mécanismes & Intentionnels & Spontanés \\
\hline $\begin{array}{l}\text { Spécifiques } \\
\text { à une université donnée }\end{array}$ & $\begin{array}{l}\text { - Région } \\
\text { Offre de formation } \\
\text { - Collegium } \\
\text { - Choix des mécanismes incitatifs } \\
\text { et d'évaluation } \\
\text { des enseignants-chercheurs } \\
\text { - COMUE, fusion des universités } \\
\text { - Application des textes LMD } \\
\text { Choix du personnel (vaca- } \\
\text { taires / enseignants-chercheurs) } \\
\text { - Architecture } \\
\text { entre école doctorale, } \\
\text { composante, laboratoire, etc. }\end{array}$ & $\begin{array}{l}\text { Parties prenantes (pairs, attentes } \\
\text { des étudiants, attentes des profes- } \\
\text { sionnels, etc.) }\end{array}$ \\
\hline $\begin{array}{l}\text { Non spécifiques } \\
\text { à une université }\end{array}$ & $\begin{array}{l}\text { - Lois (notamment celle relatives } \\
\text { à l'enseignant-chercheur) } \\
\text { - Textes associés à la réforme } \\
\text { LMD } \\
\text { - Instance d'évaluation (HCERES, } \\
\text { ANR, CNU) }\end{array}$ & $\begin{array}{l}\text { - Marché concurrentiel (marché } \\
\text { de la formation post-bac) } \\
\text { - Marché des financements de la } \\
\text { - Marché de l'emploi }\end{array}$ \\
\hline
\end{tabular}

\section{Conclusion}

Depuis le décret de 1984, l'une des missions principales des enseignants-chercheurs universitaires est de produire de la connaissance scientifique et de la diffuser. En sciences de gestion et du management, les enseignants-chercheurs doivent relever le défi d'assurer ces missions pour plus de 135000 étudiants malgré un sous-encadrement global chronique de la discipline. La latitude d'action laissée dans l'accomplissement de leur mission et ce sous-encadrement ouvrent la voie à différentes représentations du métier et, bien souvent, au risque de devoir hiérarchiser les activités prioritaires entre l'enseignement et la recherche. Les mécanismes de gouvernance du système universitaire contribuent à accentuer un tel risque, qu'ils s'agissent des lois ou décrets; des marchés de la formation, du financement ou du travail des ECG ; des attentes des parties prenantes ou du système incitatif. À ce jour, il nous semble que seul le CNU reste le garant d'une articulation simultanée des missions de production et de diffusion de la connaissance en gestion. Il constitue la seule instance de régulation à considérer toutes les missions d'un ECG, y compris dans l'évolution de sa situation professionnelle. Si des mécanismes locaux de régulation existent (conseil académique et comité de sélection), les moyens mis à leur disposition restent très limités et dépendent de la taille des universités.

La force du CNU réside aussi dans sa composition : des ECG en poste dans de plus petites universités, ou des établissements pour lesquels les sciences de gestion et du 
management sont relativement périphériques, y sont représentés. Il contribue par sa représentation à gommer l'importance plus ou moins grande accordée à notre discipline, très variable selon les universités. Or, cette disparité favorise aussi le cloisonnement : dans un établissement où la gestion est une discipline majeure, des moyens financiers ou humains y seront davantage investis afin que l'ECG puisse se concentrer sur ses missions d'enseignement et de recherche ; I'ECG en poste dans une université où la gestion est une discipline parmi beaucoup d'autres risque aussi de s'estimer insuffisamment représenté en cas de disparition de cette instance. L'évolution des missions attribuées au CNU, à la suite de la loi de programmation pluriannuelle pour la recherche (2020) (suppression de la qualification pour les professeurs des universités), remet en cause en partie ce rôle de mécanisme d'évaluation et d'incitation pour l'articulation des missions de production et de diffusion des connaissances.

En outre, d'autres voies non exhaustives pour (ré)concilier ces missions sont à explorer en complément : le choix de méthodologies de recherche spécifiques (recherche action ou recherche intervention); les politiques éditoriales des revues scientifiques en intégrant dans les publications les modalités de diffusion de la connaissance produite ; la rémunération et les primes des enseignants-chercheurs plus incitatives; l'évaluation moins segmentée des structures universitaires par les tutelles ou les agences; l'organisation plus intégrée des établissements ; l'octroi de financements portant sur les volets pédagogique et scientifique; le rapprochement des associations de formations universitaires et les associations scientifiques. Reste la question éminemment récurrente du sous-encadrement des ECG dans les universités, qui est de nature à déséquilibrer l'engagement simultané dans chacune des dimensions de leur métier. Cette question relève des stratégies des établissements et des choix réalisés lors des campagnes d’emplois. 



\title{
L'innovation pédagogique à l'université Dispositifs de mise en œuvre et de valorisation
}

\author{
Nathalie OrıOL et Mickaël DAvID
}

Ces dernières années, l'université française a été marquée par une tendance aux expérimentations pédagogiques sous l'impulsion d'une politique d'appel à projets liée à la transformation numérique (Dulbecco et al., 2018). La digitalisation a opéré une rupture tant sur le contenu, la manière d'enseigner que sur les moyens (Ghozlane et al., 2016). Le changement est profond car il sous-tend des dispositifs centrés sur l'apprenant, favorisant le blended learning. Aux côtés des impulsions favorisées par le ministère de l'Enseignement supérieur, de la Recherche et de l'Innovation (MESRI) depuis plusieurs années, la crise sanitaire a accéléré ce processus de transformation au regard de l'urgence du maintien de la continuité des enseignements. Une réflexion plus profonde trouve cependant racine au sein des enjeux majeurs sociaux et sociétaux que représente la transition vers de nouveaux modes d'apprentissages jugés plus efficaces, tant sur le plan de l'inclusion des étudiants que de leur insertion professionnelle. Selon Dulbecco et al. (2018), les transformations reposent sur une double priorité : celle de " faciliter l'accès pour tous les publics, aux études sous des formats pouvant être adaptés et individualisés " et de "faire face aux enjeux d'une société qui valorise l'acquisition de compétences plus que de savoir, ainsi que l'adaptabilité des individus ". S'opère ainsi un véritable changement de paradigme qui s'éloigne de l'idée d'une transmission " passive » du savoir et qui s'associe pleinement aux enjeux de développement de blocs de compétences (Brillet et Scouarnec, 2020). Ainsi, l'innovation pédagogique peut être considérée comme une mission d'amélioration constante des apprentissages. Mais elle peut également être considérée comme le moyen de mobiliser des savoir-faire méthodologiques en recherche et donc un instrument essentiel de formation continue, de réflexivité et de regards critiques sur nos pratiques d'enseignant-chercheur.

En définitive, l'émergence de nouvelles pratiques est une tendance croissante qu'il est nécessaire de reconnaitre et de valoriser. Mais la nature fragmentée et hétérogène des initiatives freine à la fois la lisibilité et la généralisation des innovations pédagogiques. Le CNU, dans la section 06 , est garant d'un référentiel 
commun et équilibré des différentes missions associées aux métiers et carrières des enseignants-chercheurs en sciences de gestion et du management. L'objectif du chapitre est ainsi de proposer une réflexion autour des critères d'évaluation de l'engagement attendu d'un enseignant-chercheur dans l'innovation pédagogique. Ces balises d'évaluation devront toutefois être appréhendées de manière contextuelle. Les ressources et incitations - qu'elles soient techniques, organisationnelles, humaines ou financières - disponibles pour soutenir l'innovation pédagogique sont très variables d'un établissement à l'autre. Sur ce point, comme sur les autres volets d'évaluation du CNU, l'évaluation qualitative et contextuelle est nécessaire pour apprécier l'engagement d'un collègue dans la pédagogie. Dans une première partie, nous proposons un état des lieux des nouveaux paradigmes et canevas de la pédagogie. Au sein d'une seconde partie, nous proposons d'établir un cadre d'évaluation permettant à la communauté d'objectiver et donc de valoriser leurs livrables en termes d'innovation pédagogique.

\section{État des lieux de l'innovation pédagogique}

Les fondements des pédagogies dites actives et/ou innovantes à l'université trouvent racine au sein de nombreux travaux en sciences de l'éducation, dont la mise en application essaime et se concrétise depuis quelques années en France. Pour autant, il reste parfois difficile de délimiter la frontière entre une utilisation d'une technologie innovante, une utilisation innovante de technologie et une pédagogie innovante. Comme le soulignent Youssef et Audran (2019), l'apprentissage n'est

ni linéaire, ni uniforme, ni unidirectionnel. L'innovation pédagogique doit tenir compte de ceci en permettant à l'apprenant lui-même de mettre à l'épreuve ses connaissances sans que celles-ci dépendant d'une innovation imposée de manière algorithmique.

La transformation numérique a offert un ensemble d'outils nouveaux dont a su se saisir la communauté des enseignants-chercheurs à l'université. L'innovation pédagogique s'appuie ainsi sur leur utilisation, à des fins de promotion de nouvelles conceptions de l'enseignement répondant à la diversité cognitive des apprenants et améliorant ainsi les apprentissages. Cette section a pour objectif d'établir une liste des principales innovations de procédés, de méthodes et de techniques d'animation, en lien avec les nouveaux outils de diffusion du savoir.

\section{Innovation des méthodes d'apprentissage}

Dans un esprit d'inclusion et d'interactivité avec les apprenants, la conception universelle d'apprentissage (CUA) offre un canevas pour la création de buts, de méthodes, d'évaluations et de matériel éducatif qui fonctionnent pour tous les individus (Rose et Meyer, 2002). La base de réflexion est de réaliser un alignement pédagogique et constructif entre le contenu, l'évaluation et l'acquisition de compétences par le biais d'une scénarisation du cours (Biggs et Tang, 2011). Les attendus concrets de cette conception sont : de favoriser la transversalité des compétences; de développer l'adhésion et donc l'implication des étudiants au sein des programmes; d'élargir le nombre de bénéficiaires tout en facilitant la création de communautés d'apprenants; et de proposer, dans la mesure du possible, des parcours personnalisés 
notamment en termes d'acquisition de blocs de compétences en lien avec les attendus professionnels et sociétaux.

Brillet et Scouarnec (2020) identifient huit méta-compétences permettant de délimiter les attentes professionnelles dans le domaine du management et de la gestion : (1) veiller-anticiper; (2) piloter-gérer; (3) contrôler-mesurer; (4) animer-fédérer ; (5) développer-innover ; (6) appliquer-respecter ; (7) accompagner-conseiller; et (8) communiquer-marketer. Chacune de ces méta-compétences repose sur l'articulation entre savoir-faire disciplinaires (dans les sous-domaines standardisés tels que la gestion des ressources humaines, les systèmes d'information, la finance, la logistique, la comptabilité, la stratégie, l'organisation, ou le marketing), savoir-faire transverses (maîtrise bureautique, des langues, de la recherche documentaire, de la formalisation d'un projet professionnel, etc.), mais également d'éléments de savoir-être (agilité, curiosité, pensée critique, esprit d’analyse, proactivité, etc.).

À titre d'illustration des méthodes de scénarisation permettant cet alignement constructif, les approches par projet $\left(A_{P} P_{J}\right)$, les approches par problème $\left(A_{P P}\right)$ et les jeux sérieux font figures de canevas de plus en plus utilisés par la communauté. L'APP, englobe toute séquence d'apprentissage qui doit déboucher sur une production à délivrer, laissant la liberté à tout apprenant d'y apporter sa valeur ajoutée selon son degré intérêt et ses propres représentations (exemple : réalisation d'un podcast sur un sujet d'actualité). Cette scénarisation se prête particulièrement à un objectif de facilitation de l'insertion professionnelle à un niveau Master. Le principe de I'APP, est de rendre l'étudiant actif et responsable de ses apprentissages en le mettant en contexte professionnel. Dans cet environnement, l'enseignant-chercheur tient le rôle de guide (accompagnement et feedback). La mise en place d'une classe flexible, favorisant les interactions, est essentielle. Cette pédagogie active permet à la fois d'ancrer des techniques professionnelles, mais aussi de développer le savoir être de l'apprenant (planification, communication, rédaction, coordination, persuasion). Les sciences de gestion et du management sont particulièrement adaptées à ce canevas, au sens où il s'agit pour les apprenants de développer des compétences tacites et explicites en gestion de projet et entrepreneuriat (Fayolle et Verzat, 2009 ; Oriol, 2020).

L’APP $_{M}$ est également une méthode de scénarisation pédagogique fondée sur l'apprenant et lui laissant pleine responsabilisation dans la réalisation d'objectifs. C'est un canevas qui favorise la transdisciplinarité en ce sens qu'il vise à élaborer un ensemble de solutions à apporter à une problématique complexe. Elle a notamment été adoptée de manière pionnière dans le contexte de la formation médicale, afin de favoriser les échanges entre différentes spécialités et donc la prise en charge systémique des patients. Comparativement à l'APP, elle est particulièrement fondée sur des activités de recherche d'informations et d'acquisition des expertises requises, mais toujours dans une dimension potentiellement collaborative et de co-construction (investigation, écoute active, coordination, diagnostic, solution). Au sein d'équipe d'apprenants, elle est en effet propice à l'activation et au partage des savoirs antérieurs. Ici, l'objectif n'est pas de s'articuler autour de production à réaliser, mais de savoirs et d'expertises à coordonner.

Le jeu sérieux est une autre forme de pédagogie active mais également une innovation dite techno-pédagogique liée à la ludification des apprentissages (pour une étude détaillée des liens entre l'innovation pédagogique, la gamification et la 
ludification, voir le chapitre d'Élodie Gardet et Caroline Riché dans le présent ouvrage). Ils sont fondés sur le développement d'une stratégie pédagogique inclusive grâce aux univers décontextualisés proposés par les jeux. Selon Michel et Mc Namara (2014)

ils utilisent les leviers de motivation du game design (tels que la compétition, la curiosité, la collaboration, le défi individuel) et les différents supports de jeu (depuis le jeu de plateau utilisé en présentiel jusqu'au jeu vidéo et ses avatars en immersion dans des environnements 3D) pour accroître la motivation des participants à s'engager dans des tâches jugées complexes ou rébarbatives.

En sciences de gestion et du management, les initiatives de ludification sont utilisées aujourd'hui dans une diversité de domaines professionnels tels que le marketing, le recrutement, l'innovation ou encore le management de projet (Chollet, 2019). L'apprentissage est facilité par la décontextualisation et l'immersion dans un univers virtuel propice à la concentration et à l'engagement.

\section{Innovation des modalités de diffusion des savoirs}

Le caractère agile et inclusif du projet pédagogique est étroitement lié à la manière dont les activités sont déployées au sein d'espaces d'apprentissage physiques et virtuels (Oblinger, 2006). La crise sanitaire a particulièrement bouleversé les pratiques et généré un ensemble de questions autour de l'efficacité des modalités d'enseignement. Il convient alors de distinguer la notion de comodalité de celle d'hybridation. Selon Vangrunderbeek (2020)

un cours hybride se caractérise notamment par une alternance d'activités proposée par l'enseignant.e à l'ensemble des étudiant.e.s (en présence ou à distance synchrone et asynchrone). Un cours comodal se distingue par une conception guidée par une expérience simultanée des activités en face à face et des activités synchrones au choix des étudiant.e.s.

Ainsi, la comodalité implique la construction conjointe d'activités réelles et virtuelles (par exemple l'enregistrement et la diffusion en streaming d'un cours en présentiel), tandis que l'hybridation est un continuum d'activités proposées selon différentes modalités.

En ce sens, la comodalité est moins une innovation de rupture sur le plan pédagogique que l'hybridation, mais elle repose sur un investissement important en termes d'équipement des espaces d'apprentissage (tablettes, caméra ciblante, micros déportés, etc.), d'accompagnement technique et de soutien des systèmes d'information (connexion wifi, environnement virtuel de cours, plateforme d'hébergement des vidéos en streaming ou en asynchrone, etc.). La formation hybride demande une réorganisation des scénarios pédagogiques en lien avec la transformation digitale. La possibilité de s'impliquer dans cette réorganisation est dépendante d'un cadre incitatif plus global et d'un accompagnement pédagogique individualisé (ingénieur pédagogique, processus de formation continue pédagogique, référentiel incitatif lié à la transformation, etc.). Dans les deux cas, le degré de flexibilité pédagogique est grand, impliquant une solide agilité de l'enseignant afin de conserver l'inclusion de tous les suivis d'apprentissage. 
Au sein de ces transformations, les espaces physiques sont repensés en lien avec cette logique de classe interactive et active. L'émergence de nouveaux espaces d'apprentissage nécessite également le soutien des établissements sur le plan financier. Les learning labs sont des salles de cours reconfigurées sur la base de mobilier permettant modularité et classe flexible (chaises nomades, tableaux écritoires mobiles, etc.), et sur des équipements multimédias (vidéoprojecteur interactif, écrans tactiles et tablettes, positionnement d'écrans sur plusieurs murs, etc.). Les fablabs (abréviation de fabrication laboratory) sont, quant à eux, des espaces de prototypages d'objets physiques qui peuvent aller de la simple découverte du " pouvoir de faire » à de véritables APP, disciplinaires ou transdisciplinaires.

\section{Innovation des procédés et techniques d'animation et d'évaluation}

Dans le cadre de la CUA des enseignements, les activités doivent à la fois respecter la progression des compétences envisagées, mais également préparer à l'évaluation de ces compétences. Elles doivent être suffisamment variées pour permettre de correspondre à différentes formes de raisonnement et permettre ainsi à chaque apprenant d'utiliser leurs propres schémas mentaux comme base de travail. À l'échelle d'une maquette, l'identification des acquis d'apprentissage intermédiaires et principaux, formulés à l'aide de la taxonomie de Bloom (1956) lors de la scénarisation de l'enseignement, permet également de déterminer comment la formation vient nourrir l'approche par compétence globale d'un diplôme. À titre d'illustration de ces innovations dites "de procédé ", nous pouvons citer le principe de la classe inversée (flipped classroom) consistant à rendre autonome le suivi de la partie magistrale d'un cours (par exemple avec la mise à disposition de ressources pédagogiques à investir), et privilégier, lors de séances de regroupement en interaction enseignants / apprenants, l'élaboration de discussions, tests et autres formes d'activités de feedbacks ou de positionnement. Citons également le procédé de la classe renversée qui consiste à demander aux apprenants de travailler sur l'appropriation des savoirs en toute autonomie et de se porter eux-mêmes garants de leur restitution auprès du reste de la cohorte, l'enseignant se positionnant ici en guide.

Associées à l'ensemble des innovations, la mise en place d'activités collaboratives en ligne, ou E-tivities au sens de Salmon (2002), constitue un défi important de transformation pour la communauté. À ce titre, il existe de nombreuses applications (ou modules d'extension sur Moodle) permettant de développer des activités d'évaluation, des activités de mémorisation et de synthétisation (cartes heuristiques, infographie, nuages de mots, etc.), des activités ludo-pédagogiques (jeux de sociétés, de mémoire, de logique autour des concepts clés du cours, etc.), des activités de mutualisation (portfolio numérique, glossaire collaboratif, wiki de cours, pédagothèques, etc.), de rétroaction (audio, vidéo, par les pairs, etc.), ou encore des activités de transmission (conception de capsules vidéos, présentation en ligne, animations, etc.).

Enfin, la place et le rôle de l'évaluation peut-être aussi repensé et conduire à des innovations de procédé. Le stealth assessment, ou évaluation « indolore " selon Shute et Kim (2014), repose sur un projet de progression individualisée de l'étudiant. L'évaluation devient un moteur d'amélioration en contrôle continu en lien avec la mise en place d'un portfolio de compétences. Cet alignement entre formation et approche par compétence est conçu dans un objectif d'accompagnement à l'insertion 
professionnelle et permet aux apprenants de s'autonomiser vis-à-vis d'une évaluation constante de leurs acquis. À l'issue de leur formation, ils sont ainsi en mesure d'objectiver leur expertise technique (savoir-faire disciplinaires) liée au contenu de la formation, mais également d'expliciter un ensemble de compétences tacites liées aux modalités de dispense de ces contenus et à leur implication dans le processus d'apprentissage (savoir-être et savoir-faire transverses).

\section{Faits observables de l'innovation pédagogique en sciences de gestion et du management}

Cette seconde partie se propose d'aiguiller la communauté vers un certain nombre de dispositifs existants permettant d'abriter la réflexion et les initiatives en matière d’innovation pédagogique.

\section{Écrins et vecteurs de l'innovation pédagogique}

L'opérationnalisation de l'innovation pédagogique se réalise à la fois dans le cadre de recherches scientifiques menées par des enseignants-chercheurs en sciences de gestion et du management et dans le cadre de nos pratiques d'enseignement au sein de nos établissements.

\section{Espaces de réflexion scientifique sur l'innovation pédagogique}

La réflexion sur l'innovation pédagogique est portée par les enseignants-chercheurs au sein même de leur propre discipline. Les sciences de gestion et du management produisent régulièrement des travaux doctoraux en la matière. Certaines thèses portent sur la didactique d'un concept, la décision (Kéradec, 2012) ou l'entrepreneuriat (Bouslikahne, 2011), tandis que d'autres vont s'intéresser à l'efficacité d'un dispositif pédagogique, tels que les MOOC (Cascioli, 2020). Enfin, d'autres travaux vont s'intéresser aux processus d'innovation pédagogique à l'université, notamment sur leurs ressorts intrapreneuriaux (Ewango-Chatelet, 2017). Au-delà de ces travaux doctoraux, de nombreux articles sont publiés dans les revues en sciences de gestion et du management et des communications sont régulièrement présentées lors de conférences. Ainsi, la revue Management \& Avenir, pour ne citer qu'elle, publie régulièrement des articles sur l'innovation pédagogique s'intéressant notamment à la résistance des apprenants à une innovation (Ait-Taleb et Mani, 2019), à l'usage ou la performance d'un nouveau dispositif, tels que les Open Badges (Cieply et Grand, 2019), à l'utilisation de la réalité virtuelle comme outil pédagogique dans l'enseignement supérieur (Weber et al., 2019), ou encore aux hackathons (GreselleZaïbet et al., 2018).

Plusieurs associations scientifiques de la discipline proposent des sessions spécifiques lors de leur conférence annuelle, voire des groupes de travail thématiques en leur sein, sur la question de la transformation et de l'innovation pédagogique, tel que l'AIMS (Méthodes et approches créatives et critiques de l'apprentissage du management), I'AIM (Transformation pédagogique), I'AGRH (Innovations pédagogiques), EURAM (Pedagogical innovation and research in management education), 
I'AFC (Enseignement de la comptabilité), EGOS (Generativity and inclusivity through engaged scholarship: connecting theory, methods, and praxis), l'ADERSE (Impacts de la crise sur les pratiques d'enseignement), l'AEI (La performance de l'éducation à l'entrepreneuriat en question), I'ATLAS-AFMI (Cas pédagogiques) ou l'AOM (Teaching and learning conference). De manière concomitante, les communautés scientifiques et d'expertises en sciences de gestion et du management se dotent de prix et de labels pédagogiques : citons ainsi le partenariat entre l'AFMAT, I'ATLAS-AFMI, I'AFM, I'AFFI et RIODD avec la Centrale des cas et médias pédagogiques, ou les différents dispositifs de valorisation de la FNEGE (prix du meilleur dispositif pédagogique, prix de l'innovation pédagogique en sciences de gestion et du management, labellisation).

\section{Organisation de l'innovation pédagogique dans les établissements}

Au-delà de ce travail scientifique, les établissements et l'enseignement supérieur se structurent pour encourager et accompagner les innovations pédagogiques. Nos universités s'étoffent en ingénieurs pédagogiques constituant généralement un nouveau service à part entière, ou rattaché à la direction des études et de la formation, au sein des services centraux. Ces services proposent de plus en plus des (auto-)formations, ateliers ou espaces d'échanges de pratiques sur l'innovation pédagogique. Citons également le fait que l'innovation pédagogique au sein d'une formation est un item de l'évaluation d'une formation par l'HCERES, portant ainsi une démarche qualité qui structure et incite les initiatives.

Le travail d'innovation pédagogique est également reconnu au niveau ministériel, avec notamment la création en 2019 d'un congé pour projet pédagogique (CPP) avec des mécanismes proches de ceux du congé de recherche et de conversion thématique (CRCT). Les dossiers déposés auprès de l'établissement d'affectation sont appréciés particulièrement au regard de leur dimension novatrice, de l'accompagnement à la réussite des étudiants, de créations de nouveaux contenus ou de transformation des pratiques pédagogiques notamment via l'usage d'outils numériques.

\section{Référentiel de l'innovation pédagogique}

Les critères d'évaluation des pratiques innovantes peuvent reposer sur trois piliers fondamentaux au cœur du métier d'enseignant-chercheur (tableau 1). Premièrement, l'objectif de ces innovations doit permettre de répondre plus efficacement aux missions confiées aux enseignants-chercheurs, notamment en termes d'insertion professionnelle, d'inclusion et d'accompagnement personnalisé des publics. Deuxièmement, le métier d'enseignant-chercheur repose sur le principe de partage et de transmission; la diffusion et la valorisation de ces pratiques innovantes est donc un attendu important. Enfin, troisièmement, le métier d'enseignant-chercheur repose sur l'esprit scientifique et donc le questionnement et l'approfondissement de ces connaissances issues d'autres disciplines, telles que les sciences de l'éducation, pour l'enseignement des sciences de gestion et du management au regard des spécificités des publics concernés, de leurs attentes et des attendus du marché de l'emploi sur envers eux. 
Tableau 1 : Piliers d'évaluation de l'innovation pédagogique

\begin{tabular}{|c|c|}
\hline Piliers de l'évaluation & Sous-items \\
\hline \multirow{3}{*}{ Agilité / inclusion } & $\begin{array}{l}\text { Diversité des modalités (public, formations, lieux, ouverture inter- } \\
\text { nationale, hybride, etc.) }\end{array}$ \\
\hline & Capacité à instaurer un accompagnement personnalisé \\
\hline & $\begin{array}{l}\text { Conduite de dispositifs d'aide à l'insertion professionnelle et ados- } \\
\text { sement à une approche par compétences }\end{array}$ \\
\hline \multirow{3}{*}{ Diffusion / valorisation } & $\begin{array}{l}\text { Diffusion et réplication des démarches entreprises auprès d'autres } \\
\text { collègues et composantes }\end{array}$ \\
\hline & $\begin{array}{l}\text { Notoriété et sélectivité des supports éditoriaux adoptés pour leur } \\
\text { publication }\end{array}$ \\
\hline & Production de supports pédagogiques standardisés \\
\hline \multirow{3}{*}{ Synergies avec la recherche } & $\begin{array}{l}\text { Insertion dans la communauté de recherche en sciences de gestion } \\
\text { et du management dédiée à la pédagogie }\end{array}$ \\
\hline & $\begin{array}{l}\text { Capacité à mobiliser de nouvelles problématiques / propositions de } \\
\text { lecture }\end{array}$ \\
\hline & $\begin{array}{l}\text { Développement de cas pédagogiques ancrés sur les axes de re- } \\
\text { cherche }\end{array}$ \\
\hline
\end{tabular}

\section{Faits observables et indices d'appréciation}

Le tableau 2 présente une liste de faits observables et d'indices d'appréciation proposés afin de valoriser l'engagement d'un enseignant-chercheur dans l'innovation pédagogique. Ce tableau n'engage pas le $\mathrm{CNU}$, mais seulement les auteurs du présent chapitre, et a vocation à aider les enseignants-chercheurs à expliciter leur engagement dans l'innovation pédagogique lorsqu'ils déposent un dossier auprès du CNU ou de leurs instances locales.

Tableau 2 : Faits observables de l'innovation pédagogique

\begin{tabular}{|c|c|c|}
\hline Champs & Faits observables & Indices d'appréciation \\
\hline \multirow{3}{*}{$\begin{array}{l}\text { Réflexivité individuelle } \\
\text { - Attendu que l'enseignant- } \\
\text { chercheur interroge } \\
\text { régulièrement ses pratiques }\end{array}$} & $\begin{array}{l}\text { Participation à des formations } \\
\text { continues : allant d'une } \\
\text { conférence à une formation } \\
\text { diplômante }\end{array}$ & Nombre et volume horaire \\
\hline & $\begin{array}{l}\text { Construction et animation de } \\
\text { formation }\end{array}$ & $\begin{array}{l}\text { Nombre, volume horaire et } \\
\text { itération Dispensation au-delà } \\
\text { de son établissement }\end{array}$ \\
\hline & $\begin{array}{l}\text { Obtention d'un congé pour } \\
\text { projet pédagogique (CPP, } \\
\text { équivalent du CRCT), obtention } \\
\text { de primes de transformation } \\
\text { numérique }\end{array}$ & $\begin{array}{l}\text { Nombre, modalités d'obtention } \\
\text { et montants }\end{array}$ \\
\hline
\end{tabular}




\begin{tabular}{|c|c|c|}
\hline \multirow{4}{*}{$\begin{array}{l}\text { Initiatives de transformation } \\
\text { des pratiques individuelles } \\
\text { - Attendu que l'enseignant- } \\
\text { chercheur fasse évoluer ses } \\
\text { dispositifs d'enseignement }\end{array}$} & $\begin{array}{l}\text { Création et mise à disposition } \\
\text { de ressources pédagogiques, } \\
\text { type } \mathrm{MOOC} \text {, étude de cas, etc. }\end{array}$ & $\begin{array}{l}\text { Nombre, indicateur } \\
\text { d'utilisation de ces ressources }\end{array}$ \\
\hline & $\begin{array}{l}\text { Maquette de formation et } \\
\text { syllabus de cours }\end{array}$ & $\begin{array}{l}\text { Degré d'innovation et } \\
\text { pertinence du dispositif } \\
\text { innovant dans le cadre d'un } \\
\text { cours ou d'une formation }\end{array}$ \\
\hline & $\begin{array}{l}\text { Participation à des appels à } \\
\text { projet pédagogique, concours } \\
\text { entre étudiants, hackathon etc. }\end{array}$ & $\begin{array}{l}\text { Nombre de participations, } \\
\text { ambition des objectifs, nombre } \\
\text { d'étudiants concernés }\end{array}$ \\
\hline & Obtention de prix et distinction & $\begin{array}{l}\text { Nombre, rayonnement de } \\
\text { l'organisme délivrant le prix }\end{array}$ \\
\hline \multirow{4}{*}{$\begin{array}{l}\text { Initiatives de transformation } \\
\text { des structures et pratiques } \\
\text { collectives - Attendu que } \\
\text { l'enseignant-chercheur prenne } \\
\text { des initiatives pour faire évoluer } \\
\text { et structurer la communauté, } \\
\text { notamment lorsqu'il est en } \\
\text { situation de responsabilité }\end{array}$} & $\begin{array}{l}\text { Participation à des comités } \\
\text { d'évaluation HCERES de } \\
\text { formation }\end{array}$ & Nombre, rôle dans l'évaluation \\
\hline & $\begin{array}{l}\text { Participation à des projets de } \\
\text { transformation pédagogique }\end{array}$ & $\begin{array}{l}\text { Rôle dans le projet, ampleur et } \\
\text { rayonnement du projet }\end{array}$ \\
\hline & $\begin{array}{l}\text { Responsabilité pédagogique } \\
\text { au sein des établissements et } \\
\text { autres structures }\end{array}$ & $\begin{array}{l}\text { Niveau de responsabilité, } \\
\text { ampleur et rayonnement } \\
\text { de la structure, durée de la } \\
\text { responsabilité }\end{array}$ \\
\hline & $\begin{array}{l}\text { Obtention / gestion } \\
\text { de financement pour } \\
\text { investissement à caractère } \\
\text { pédagogique }\end{array}$ & $\begin{array}{l}\text { Type, montant, modalités } \\
\text { d'obtention, objet }\end{array}$ \\
\hline \multirow{3}{*}{$\begin{array}{l}\text { Production de connaissances } \\
\text { - Attendu que l'enseignant- } \\
\text { chercheur mobilise ses } \\
\text { savoir-faire en recherche pour } \\
\text { produire de la connaissance en } \\
\text { matière d'enseignement de la } \\
\text { gestion }\end{array}$} & $\begin{array}{l}\text { Articles, communications et } \\
\text { chapitres d'ouvrage }\end{array}$ & $\begin{array}{l}\text { Nombre, contribution } \\
\text { scientifique, nombre de } \\
\text { citations }\end{array}$ \\
\hline & $\begin{array}{l}\text { Encadrement de mémoire, } \\
\text { thèse ou HDR sur le sujet }\end{array}$ & $\begin{array}{l}\text { Nombre, niveau, contribution } \\
\text { scientifique, \% d'encadrement }\end{array}$ \\
\hline & $\begin{array}{l}\text { Participation à des projets de } \\
\text { recherche }\end{array}$ & $\begin{array}{l}\text { Rôle dans le projet, ampleur } \\
\text { et rayonnement du projet, } \\
\text { durée du projet, contribution } \\
\text { scientifique du projet }\end{array}$ \\
\hline \multirow{2}{*}{$\begin{array}{l}\text { Animation et structuration de } \\
\text { la communauté en matière de } \\
\text { recherche sur l'enseignement } \\
\text { de la gestion - Attendu que } \\
\text { l'enseignant-chercheur prenne } \\
\text { des initiatives pour faire évoluer } \\
\text { et structurer la communauté } \\
\text { quant à la production de } \\
\text { connaissances la matière }\end{array}$} & $\begin{array}{l}\text { Animation de sessions ou } \\
\text { numéro spécial de revue sur la } \\
\text { thématique }\end{array}$ & $\begin{array}{l}\text { Rôle dans la session, } \\
\text { rayonnement de la revue ou } \\
\text { de la conférence, nombre de } \\
\text { papiers évalués }\end{array}$ \\
\hline & $\begin{array}{l}\text { Responsabilité au sein } \\
\text { d'association ou revue dédiée }\end{array}$ & $\begin{array}{l}\text { Niveau de responsabilité, } \\
\text { ampleur et rayonnement de la } \\
\text { structure }\end{array}$ \\
\hline
\end{tabular}

\section{Champs de faits observables}

Les enseignants-chercheurs peuvent contribuer à l'innovation pédagogique sur les trois dimensions de leur métier : l'enseignement, la recherche et les responsabilités collectives. Au regard de ces trois dimensions et de leur imbrication, nous proposons cinq champs d'engagement d'un enseignant-chercheur dans l'innovation pédagogique (figure 1) : 
- L'engagement dans la réflexivité individuelle. Du fait de la relative autonomie d'un enseignant-chercheur, tant dans le contenu que les méthodes de ses enseignements, on peut en effet attendre qu'il interroge régulièrement ses pratiques. Cette réflexivité individuelle peut s'observer par le fait de suivre ou de dispenser des formations sur l'innovation pédagogique, ou d'avoir obtenu des congés ou primes spécifiques.

- L'engagement dans la modification des pratiques individuelles d'enseignement. Cet engagement peut s'observer par la création de ressources pédagogiques (MOOC, étude de cas, etc.), par la création et mise à disposition de maquette de formation et syllabus de cours innovants (en termes de contenu, de scénarisation, etc.), et par la participation à des appels à projet pédagogique ou l'obtention de distinction.

- L'engagement dans la transformation des structures et pratiques collectives. Un enseignant-chercheur peut avoir un rôle déterminant dans l'innovation pédagogique lorsqu'il est en responsabilité dans un établissement, notamment lorsqu'il s'agit d'animer la construction périodique d'une offre de formation ou de monter des dossiers de réponse à des appels à projet à caractère pédagogique, mais aussi dans l'animation au quotidien d'une équipe pédagogique ou des services supports.

- L'engagement dans la production de connaissances en matière d'enseignement des sciences de gestion et du management. Par esprit scientifique, les enseignants-chercheurs peuvent vouloir mobiliser leurs savoir-faire méthodologiques pour questionner et approfondir les connaissances issues des sciences de l'éducation dans l'enseignement des sciences de gestion et du management. Il appartient ainsi à la communauté de s'engager dans des recherches en ce sens.

- L'engagement dans l'animation et la structuration de la recherche sur l'enseignement des sciences de gestion et du management. La recherche est une activité collective. Créer et développer une nouvelle communauté sur ces questions et la faire vivre est donc une activité nécessaire à l'innovation pédagogique pertinente. Les faits observables de ce dernier champ d'action sont donc l'animation de sessions ou numéros de revues sur la thématique ou encore la responsabilité au sein d'association ou revue dédiées.

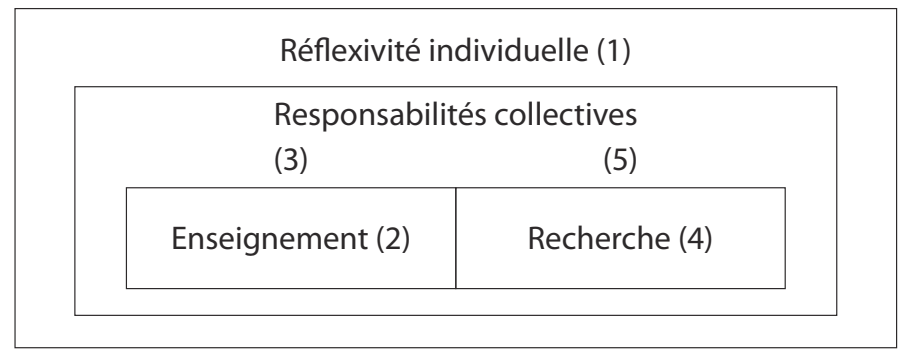

Figure 1 : Les champs d'engagement d'un enseignant-chercheur dans I'innovation pédagogique 


\section{Indices d'appréciation}

Les différents faits observables présentés dans le tableau 2 doivent pouvoir être pondérés et comparés. Les indices d'appréciation que nous proposons dans ce tableau ne sont pas exhaustifs et doivent prendre en compte les contextes hétérogènes dans lequel les enseignants-chercheurs exercent leur fonction. L'objectif d'une telle liste est d'aider la communauté à objectiver et valoriser leur implication dans l'innovation pédagogique.

\section{Conclusion}

L'innovation pédagogique est au cœur de attendus légitimes envers un enseignant-chercheur et une nécessité organisationnelle et sociétale. Toutefois, il y a de multiples façons de s'y investir. Le présent chapitre propose un guide pour la valorisation de l'engagement d'un enseignant-chercheur en la matière. Nous invitons chaque collègue à valoriser ces faits observables dans leur CV académique et lors de toute demande d'évaluation auprès du CNU ou des instances locales, que ce soit lors d'une demande de qualification aux fonctions de maître de conférences, d'une demande d'avancement de grade ou d'un recrutement selon les articles 46.1 et 46.3. Nous plaidons également pour qu'ils soient appréciés à leur juste valeur au sein des établissements, dans une perspective d'amélioration du cadre incitatif relatif à ces missions. 



\section{Innovations pédagogiques en sciences de gestion et du management \\ Le rôle croissant de la gamification et de la ludification}

Élodie GARDEt et Caroline Rıché

Ludification, escape games pédagogiques, serious games, serious gaming, ludopédagogie, gamification, sont autant de termes qui émergent ces dernières années des dossiers de qualification et d'avancement des enseignants-chercheurs. Ces nouvelles pratiques sont désormais considérées dans leur évaluation. Les recherches sur le sujet, pour la plupart, soit s'orientent vers la présentation d'expérimentations ou d'études de cas, soit visent à analyser l'impact de ces nouvelles méthodes sur l'acquisition de compétences des étudiants (Yordanova, 2019). Toutefois, les contours de ces pratiques pédagogiques sont encore mal définis et recouvrent des réalités fortement hétérogènes (figure 1). À travers le présent chapitre, l'objectif est de proposer une synthèse des principales pratiques de ludopédagogie afin de mieux appréhender des innovations pédagogiques qui suscitent un intérêt croissant de la part des enseignants-chercheurs.

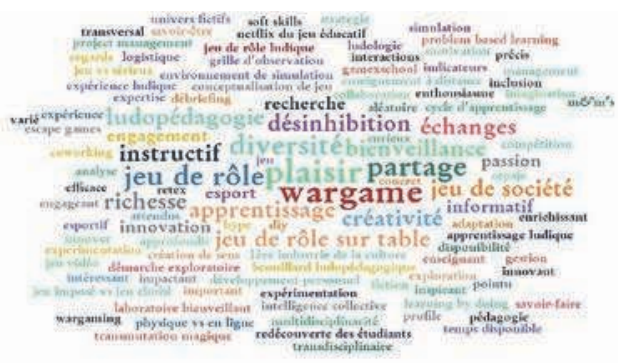

Figure 1 : Mots-clés les plus utilisés dans les actes du colloque 2021 International game evolution : management et pédagogie ludique Source : d'après la 5e édition CIGE, Montpellier, mai 2021 


\section{Ludification de l'enseignement supérieur : vers un nouveau mode d'apprentissage}

Le phénomène d'innovation pédagogique a fait l'objet de multiples recherches, à la fois en sciences de gestion et du management, en sciences de l'éducation et en sociologie. Dans ces travaux, les méthodes, leurs conceptualisations et la terminologie sous-jacente sont diverses, parfois fragmentaires, et témoignent d'un manque de consensus quant aux différents types d'innovations pédagogiques. Plus spécifiquement, les études de cas sont régulièrement assimilées à des innovations sans pour autant justifier leur dimension innovante. Nous proposons ici une typologie des pratiques les plus répandues en sciences de gestion et du management, ainsi qu'une définition des principaux termes sur le sujet.

\section{Définitions et contours}

Malgré l'intérêt croissant qu'elles provoquent, les pratiques innovantes par le jeu ne font pas l'objet d'une conceptualisation consensuelle en sciences de gestion et du management, et mobilisent une terminologie employée diversement dans la littérature (Walther et Larsen, 2020). Par conséquent, nous proposons de catégoriser et spécifier les principales pratiques décrites par les enseignants-chercheurs dans leurs dossiers de qualification ou d'avancement pour mieux les appréhender sur le plan conceptuel. Le tableau 1 permet notamment de mieux comprendre les liens et différences entre innovations pédagogiques, game-based learning, gamification et ludification.

Tableau 1 : Comparaison entre les principaux termes des pratiques innovantes

\begin{tabular}{|c|c|c|c|}
\hline & $\begin{array}{l}\text { Innovations } \\
\text { pédagogiques }\end{array}$ & Game-based learning & Ludification / Gamification \\
\hline 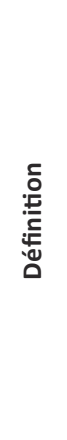 & $\begin{array}{l}\text { Toute nouvelle } \\
\text { technique d'enseigne- } \\
\text { ment, stratégie, outil } \\
\text { ou ressource d'appren- } \\
\text { tissage qui pourrait } \\
\text { être utilisée pour } \\
\text { conduire à des tech- } \\
\text { niques d'enseignement } \\
\text { efficaces qui bénéfi- } \\
\text { cient à l'apprentissage } \\
\text { et à l'engagement des } \\
\text { étudiants (Taylor et al., } \\
\text { 2018) }\end{array}$ & $\begin{array}{l}\text { La méthode pédago- } \\
\text { gique est fondée sur } \\
\text { le jeu et une pratique } \\
\text { ludique immersive. } \\
\text { Cela dépasse le simple } \\
\text { usage de mécanismes } \\
\text { du jeu permettant de } \\
\text { ludiciser un contenu } \\
\text { formel (Lépinard et } \\
\text { Vandangeon-Derumez, } \\
\text { 2019) }\end{array}$ & $\begin{array}{l}\text { Infusion de techniques de conception } \\
\text { de jeux dans des contextes non ludiques, } \\
\text { qui dérive de ludic et de -ification. Lu- } \\
\text { dique vient du mot latin ludo signifiant } \\
\text { "jouer ", tandis que le suffixe -ification } \\
\text { signifie " le processus de devenir ». En } \\
\text { résumé, introduction et amélioration } \\
\text { des éléments et des mécanismes de } \\
\text { jeu dans des objets, des installations et } \\
\text { des situations qui ne sont pas des jeux } \\
\text { (Walther et Larsen, 2020) }\end{array}$ \\
\hline 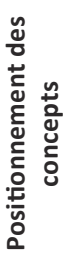 & & udification / Ga & IIL \\
\hline
\end{tabular}


Ainsi, les innovations pédagogiques se caractérisent par : (1) l'utilisation de nouveaux matériels révisés (matériel ou technologies de programme); (2) l'utilisation de nouvelles approches pédagogiques (stratégies ou activités d'enseignement) ; et (3) la modification des croyances (hypothèses pédagogiques) (Béchard et Pelletier, 2000). Le game-based learning suppose une stratégie pédagogique basée directement sur le jeu et ses mécanismes ludiques. C'est la pratique du jeu, sa contextualisation et ses moyens qui facilitent l'apprentissage (Lépinard et Vandangeon-Derumez, 2019). La gamification n'est qu'une sous-catégorie des innovations pédagogiques, qui propose d'enrichir une activité préexistante par des mécanismes motivants du jeu pour atteindre un objectif. La ludification repose, quant à elle, sur le même type d'éléments de jeu que la gamification, mais concerne spécifiquement l'amélioration des éléments de jeu. Les caractéristiques essentielles sont surtout basées sur les moyens de " concevoir » et de " raconter " des histoires d'une manière nouvelle et captivante, c'est-à-dire "ludifiée ", alors que la gamification porte davantage sur la motivation, la récompense et le retour d'information (Walther et Larsen, 2020).

Afin d'être complètement ludifié, un enseignement doit s'appuyer sur plusieurs aspects (Michel et McNamara, 2014), dont notamment : (1) avoir un sens épique : créer un monde attractif (personnage, design, sons, couleur, etc.) qui donne du sens à une action et stimule l'implication des étudiants ; (2) permettre l'accomplissement et d'être en situation de pouvoir agir : validation de compétences, de niveaux, de progression ${ }^{1}$; (3) posséder une part d'imprévisibilité et de défis (doser une part de compétences et de hasard afin de donner l'envie de découvrir la suite), nécessitant que l'étudiant saisisse l'opportunité quand elle se présente ; et (4) favoriser le travail en collaboration et/ou compétition via la connexion à une communauté. Nombreuses sont les recherches qui, pour faire reculer ce flou sémantique, proposent des critères de différenciation. Nous proposons une synthèse de ces critères, puis une typologie des pratiques.

\section{Essai de typologie}

La multitude de types d'innovations pédagogiques et la diversité des pratiques amplifient la complexité de compréhension de ces nouvelles formes d'enseignement. La première source d'une telle complexité implique de multiples types de pratiques différenciées en fonction de leurs objectifs pédagogiques, de leurs sous-disciplines, de leur public d'étudiants (IAE vs. écoles d'ingénieurs, formation initiale vs. formation continue) et de la taille des groupes. En outre, de nouveaux supports sont apparus, combinant différentes caractéristiques, telles que le degré de technologie, la nature de la relation pédagogique entre les enseignants-chercheurs et les étudiants, le type d'apprentissage attendu (coopératif, par problème, via l'approche cognitive, etc.).

Les principales typologies offertes par la littérature concernent l'objectif d'apprentissage ou la nature du support. Béchard et Pelletier (2000) proposent une typologie des innovations pédagogiques en contexte universitaire fondée sur le type d'innovations pédagogiques (technologiques, psycho-cognitives, socio-cognitives) et

1 Des récompenses sont proposées quand des compétences sont acquises (sous la forme de notes ou d'objets collectionnables par exemple), renforçant le conditionnement positif et faisant progresser l'étudiant dans sa compréhension des objectifs académiques. 
sur leurs processus. Ainsi, un projet d'innovation pédagogique pourrait se caractériser par un processus d'élaboration organisationnel en cinq étapes : des contextes à l'idée, de l'idée à l'occasion, de l'occasion au projet, du projet à la classe, de la classe à l'institution. D’autres recherches, notamment celle de Kebritchi et Hirumi (2008), s'intéressent aux types de pratiques et à l'objectif pédagogique. Elles distinguent les situations étudiées par les étudiants, le message diffusé, le type d'entraînement dispensé et le mode d'échange des connaissances (Alvarez et Djaouti, 2012).

Outre ces classifications, des typologies plus classiques en management de l'innovation peuvent également être mobilisées pour distinguer les innovations de produit (création de nouveaux supports d'enseignements), de procédé (nouvelles méthodes d'acquisition des connaissances et de compétences), d'organisation (intégration de partenaires, nouvelles pratiques en dehors de l'université) et de commercialisation (changement de format des supports pédagogiques existants) (OCDE, 2005). Par ailleurs, le degré d'intensité de l'innovation peut également être pris en compte afin d'évaluer les pratiques : innovation incrémentale vs. radicale.

La pratique de la ludopédagogie peut s'inscrire dans un enseignement contextualisé (business games, études de cas, etc.). L'objectif est alors de reproduire et simuler avec fidélité une situation professionnelle afin de faciliter le transfert de connaissances et compétences. Une innovation plus radicale reposerait sur des dispositifs pédagogiques décontextualisés grâce aux jeux de rôles ou wargames, aux univers fictionnels ou historiques. Dans ce contexte de jeu, l'étudiant est sorti du cadre classique du cours pour s'immerger dans une réalité " extraordinaire ». Cette stratégie pédagogique inclusive facilite l'engagement des étudiants (Lépinard, 2019).

Dans la lignée des travaux de Salas et al. (2009), plusieurs auteurs se sont intéressés au rôle prépondérant de la technologie dans les pratiques d'innovation des enseignements (Pasin et Giroux, 2011 ; Yordanova, 2019). Leurs travaux considèrent que la technologie est une compétence indispensable que doivent maîtriser tous les acteurs souhaitant développer des innovations pédagogiques. Toutefois, si l'impact de l'évolution technologique sur les pratiques d'innovation en milieu universitaire est indéniable, il semble que les universités peuvent développer des innovations en dehors de la technologie (Yordanova, 2019). Si ces innovations non technologiques sont ignorées par certaines recherches sur les innovations pédagogiques et la ludification, c'est en partie parce qu'elles ne sont pas toujours très tangibles. Suite à cette synthèse, nous proposons de les classifier via deux principaux axes (tableau 2) :

- Degré d'usage des nouvelles technologies. L'usage correspond au choix d'utiliser partiellement ou totalement les nouvelles technologies dans les supports pédagogiques. Il ne s'agit pas d'une décision dichotomique puisqu'elle varie en fonction du degré d'usage, lié aux compétences techniques des enseignants et à leurs disponibilités pour les acquérir. Les répercussions des nouvelles technologies sur l'enseignement varient selon l'intérêt, le savoir-faire et les objectifs propres de chaque enseignant.

- Degré de ludification. Il s'agit du niveau d'usage de la mécanique du jeu mobilisée dans les situations d'apprentissage pour résoudre des problèmes et faire participer les étudiants. La notion de ludification renvoie à un ensemble de techniques permettant de transformer un objet ou une situation " autre » en un jeu (Bonenfant et Philippette, 2018). Cet axe renvoie aux mécaniques de jeu ainsi qu'aux valeurs attribuées à cette activité ludique. La notion de ludification fait ici 
référence à l'utilisation de stratégies reconnues comme appartenant au domaine du jeu.

Tableau 2 : Essai de typologie des innovations pédagogiques

\begin{tabular}{|c|c|c|}
\hline+ & $\begin{array}{c}\text { Plateformes d'apprentissage } \\
\text { Voltaire, écri+, Karuta }\end{array}$ & $\begin{array}{c}\text { Business game } \\
\text { Degré d'usage } \\
\text { de la technologie }\end{array}$ \\
\cline { 2 - 4 } & $\begin{array}{c}\text { Challenges étudiants, } \\
\text { simulation par jeux de rôles } \\
\text { Semaine de la créativité, } \\
\text { challenge entreprise }\end{array}$ & $\begin{array}{c}\text { Jeux de plateaux et de rôles, } \\
\text { wargames, escape games } \\
\text { Créanov, jeu de dés Kanban, } \\
\text { Horizon }\end{array}$ \\
\cline { 2 - 3 } & $-\quad$ Degré de ludification \\
\hline
\end{tabular}

Les deux axes identifiés conduisent à faire ressortir quatre catégories d'innovations pédagogiques, aux caractéristiques singulières. Les challenges étudiants sont des concours étudiants généralement menés en collaboration avec des organismes externes (entreprises, organismes publics, associations, etc.), et qui permettent aux partenaires de repérer des talents, de promouvoir leurs savoir-faire et de capter de nouvelles idées. Pour les étudiants, ces challenges constituent une nouvelle voie d'apprentissage concrète, offrent aux lauréats une expérience significative attestée sur un CV et constituent une opportunité de collaborer avec, voire d'intégrer l'organisme partenaire. À titre d'illustration, la plupart des écoles d'ingénieurs organisent désormais des événements pédagogiques réunissant étudiants et entreprises autour de problématiques concrètes afin d'impulser ou développer de nouveaux projets. Les challenges constituent des moments privilégiés de rencontre et d'échange entre les étudiants, les entreprises et les enseignants. Notons que certains jeux de rôles reposent aussi sur la simulation d'une situation réelle et permettent aux étudiants de s'immerger dans une réalité professionnelle en développant notamment leur empathie et leur compréhension de la situation simulée (client difficile, situation de crise en entreprise, etc.). Bien que le dispositif favorise l'implication des étudiants, la dimension ludique n'est pas ici présente. En revanche, le format d'apprentissage facilite l'expression de chacun des étudiants, même introvertis, et une cohésion du groupe.

De leur côté, les plateformes d'apprentissage représentent une interface technique et occupent une fonction d'intermédiaire dans l'accès aux informations, contenus et services (Adobor et Daneshfar, 2006). Elles autorisent la hiérarchisation des contenus pédagogiques afin de les présenter de manière structurée et adaptée aux étudiants. Ainsi, sur le marché de l'amélioration des compétences rédactionnelles des étudiants, des plateformes telles que Voltaire ou écri+ permettent non seulement de mobiliser et utiliser des contenus pour former les étudiants en fonction de leur niveau, mais également de les tester en proposant de leur délivrer un certificat de leurs compétences.

Les jeux de plateau, de rôles, les wargames et escape games sont tous basés sur la coopération puisque, quel que soit le type de support (plateau, cartes, jetons, dés, etc.), ils conduisent à nouer des relations à plusieurs en s'amusant. Ces jeux portent souvent sur une thématique spécifique. Le jeu des dés Kanban permet d’enseigner 
la fabrication synchronisée et de simuler le passage d'une entreprise en flux poussé vers un flux tiré ; Horizon permet d'apprendre les techniques de base du management de projet (planification, maîtrise des risques, optimisation et contrôle des coûts, management d'équipe). Les jeux ont pour but de faciliter l'appropriation de compétences spécifiques par les participants, en mobilisant la dimension ludique, sur des notions pouvant être théoriquement abstraites. Le jeu de rôles peut par ailleurs proposer une dimension ludique s'il s'inscrit dans un univers décontextualisé et fictif, déconnecté de l'univers savant ; dans ce cas, il n'est pas une simulation d'une expérience professionnelle directe. Lépinard (2019) définit le wargame comme

un artefact catalyseur permettant de créer des situations managériales réalistes grâce à un système de règles, des scénarios et du matériel (pions, cartes, figurines, etc.) et représentant un environnement historique ou fictif de confrontation armée.

Cela suppose donc coopération et mise en compétition, puis une recontextualisation managériale. Enfin, les escape games pédagogiques sont des jeux d'évasion basés sur la résolution d'énigmes par une équipe d'étudiants.

Nous terminerons avec les jeux d'entreprise, ou business games. Nombreux, et de différents types, ils possèdent certaines caractéristiques communes : la prise de décision, la vue d'ensemble, l'orientation vers un objectif et l'accent mis sur la résolution de problèmes (Sawyer, 2002). Ils utilisent des ressources basées sur les technologies de l'information et de la communication (TIC) pour aborder des contenus éducatifs d'une manière plus agréable et plus interactive, et recourent à la simulation de scénarios pour appréhender l'impact de prises de décision. Ils permettent d'acquérir des compétences en matière de gestion (stratégie, ressources humaines, finance, marketing, logistique, etc.), toutes nécessaires au pilotage d'une organisation (Pando-Garcia et al., 2016).

\section{Les principaux avantages et freins des nouvelles pratiques}

Mettre en place des dispositifs de ludopédagogie est inspirant pour les enseignants et présente des intérêts certains en termes de motivation, d'apprentissage et de réussite des étudiants. L'opérationnalisation se heurte parfois à certains freins. L'objet de la section est d'exposer les enjeux des nouvelles pratiques.

\section{Pourquoi développer des innovations pédagogiques?}

Traditionnellement, les travaux pratiques en sciences de gestion et du management se fondent sur des études de cas d'entreprise ou de secteur avec pour objectif principal d'utiliser les connaissances théoriques vues en cours. Désormais, de nouvelles pratiques émergent et les étudiants les considèrent comme un outil d'apprentissage plus efficace (Farrell, 2005). Les pratiques pédagogiques innovantes stimulent l'intérêt et la motivation des étudiants, et s'avèrent efficaces pour favoriser l'inclusion et rendre l'apprentissage plus performatif. 


\section{La motivation des apprenants}

Les pédagogies innovantes, notamment les approches ludiques, peuvent améliorer la motivation et la capacité perçue des étudiants à traiter de l'information complexe de manière cognitive. De plus en plus d'universités, mais également d'entreprises, se tournent vers les business games pour former les étudiants et les futurs cadres. Ces nouvelles pédagogies permettent de promouvoir non seulement les compétences techniques (analyse financière, gestion de la marque, maîtrise du process industriel, etc.), mais également des soft skills (capacité de communication, management d'équipe, etc.). Afin de favoriser la motivation des étudiants, plusieurs mécanismes empruntés au jeu peuvent être mis en œuvre pour donner davantage de sens à l'apprentissage : définir des récompenses (matérielles ou immatérielles), définir un niveau à atteindre (pour permettre à l'étudiant de s'engager dans l'apprentissage et en fixer les attendus), proposer des tableaux avec des points et des classements (pas nécessairement en lien avec la performance ou l'atteinte de l'objectif).

Au niveau conceptuel, la théorie de l'expérience optimale, ou flow (Csikszentmihalyi, 1990), est particulièrement utilisée pour expliquer l'engagement et la motivation des étudiants dans l'utilisation des nouvelles pratiques. Cette théorie considère que l'expérience de flow, c'est-à-dire l'état de bonheur psychologique dépendant de la manière dont on vit les situations nouvelles d'apprentissage, permet aux étudiants de mieux sélectionner des informations signifiantes, d'évaluer des situations complexes et de faire des choix en conséquence. Ainsi, les étudiants sont naturellement et intrinsèquement motivés à faire ce qu'ils font (Bonenfant et Philippette, 2018). L'activité pédagogique peut comporter un ou plusieurs types de manifestations : cognitive, émotionnelle (affective), physique, perceptuelle (sensorielle) et sociale. Un fort contenu émotionnel associé à des interactions sociales nécessaires au jeu peut produire des moments d'immersion, vécus comme une expérience intense.

\section{L'inclusion par le jeu}

Dans une grande partie des situations d'apprentissage, la ludification est réfléchie comme une activité de groupe qui favoriserait la cohésion d'équipe et encouragerait les échanges et les confrontations d'idées. Une telle logique pédagogique inclusive génère plus de spontanéité chez les apprenants, un esprit d'initiative, de la créativité et de l'entraide. Elle permet de dédramatiser des situations ou des contextes d'apprentissage complexe (gestion de crise en entreprise, contexte sanitaire, peur de l'avenir, etc.). La logique inclusive permet à des publics fragiles, qui ne participent pas ou moins dans un contexte d'apprentissage plus classique, de s'aventurer en étant actifs dans le jeu. Les mécanismes du jeu lèvent en effet certains freins psychologiques et luttent contre le mauvais stress en dédramatisant l'erreur. La cohésion de groupe favorise l'entraide et la confiance en soi, et facilité aussi l'inclusion de publics en situation de handicap.

\section{Un apprentissage amélioré et personnalisé}

Les nouvelles pratiques offrent les avantages d'un apprentissage à la fois expérientiel et génératif, et sont réputées produire une expérience d'apprentissage améliorée (Pasin et Giroux, 2011). Des recherches empiriques indiquent qu'en comparaison avec 
des méthodes d'enseignement traditionnelles, les nouvelles pratiques parviennent mieux à aider les étudiants à développer des compétences telles que la résolution de problèmes complexes, la prise de décision stratégique et les compétences comportementales, notamment le travail d'équipe et l'organisation (Salas et al., 2009). Les étudiants développent une perspective plus globale et sont davantage capables de relier l'apprentissage à des situations réelles.

Ceci permet également de se rapprocher des réalités du monde économique et de l'environnement concurrentiel (Faria et al., 2009), les étudiants comprenant mieux les effets systémiques. Ils sont actifs tout au long du processus d'apprentissage, et au lieu de simplement appliquer des règles ou de restituer une théorie, les nouvelles pratiques les rendent responsables de leur propre apprentissage (Salas et al., 2009). Enfin, sachant que les courbes d'apprentissage et les manières d'apprendre diffèrent selon les étudiants, les nouvelles pratiques innovantes permettent de gérer l'hétérogénéité résultante qui s'avère essentielle pour assurer un transfert de compétences (Cavory et al., 2019). Si les avantages de la ludification des enseignements en sciences de gestion et du management sont nombreux, il est cependant nécessaire d'identifier les freins potentiels permettant de fixer des objectifs réalistes et une activité d'apprentissage constructive.

\section{Les principaux freins à la mise en œuvre des pratiques innovantes}

Les freins à la mise en œuvre des nouvelles pratiques peuvent être externes (nouvelles générations d'étudiants - génération $\mathrm{Z}$ puis alpha -, reconnaissance des pratiques dans la carrière, etc.) ou internes (manque de moyens financiers, humains, niveau d'expertise technique liée à la culture et aux comportements).

\section{Barrières externes}

Si les nouvelles générations d'étudiants sont plutôt favorables aux déploiements des pratiques innovantes, différentes formes de résistance des apprenants peuvent toutefois émerger (résistance individuelle ou collective ; résistance situationnelle ou pré-dispositionnelle; résistance passive ou active [Ait-Taleb et Mani, 2019]). Dans le cadre des business simulation games, trois catégories de facteurs de résistance ont ainsi été identifiés (Ait-Taleb et Mani, 2019) : (1) des facteurs liés aux fonctionnalités du jeu : qualité du système, complexité d'utilisation perçue, etc. ; (2) des facteurs liés au contexte d'utilisation : défaut dans la phase de formation initiale, problème de communication, connaissances antérieures insuffisantes, etc. ; et (3) des facteurs liés aux caractéristiques des apprenants : stress, expériences antérieures, personnalité de l'apprenant (confiance en soi, regard des autres, etc.).

Si des classements et des critères objectifs d'évaluation des productions scientifiques existent pour la recherche, les critères qui permettaient une meilleure reconnaissance des dispositifs pédagogiques sont moins précis (voir sur ce point le chapitre de Nathalie Oriol et Mickaël David dans le présent ouvrage). Enfin, les caractéristiques de l'organisation et son environnement, comme l'indiquent Corrocher et Fontana (2008), sont de nature à freiner ou favoriser l'implémentation de dispositifs pédagogiques innovants (filière sélective ou non, nombre d'enseignants, 
présence d'un laboratoire de recherche, effectifs et taille des groupes d'étudiants, zone géographique et couverture Internet, etc.). Si certaines institutions, portées par les contraintes de labellisation de diplômes, accordent une place importante à la stratégie d'innovation porteuse d'une image attractive auprès des publics, d'autres ne peuvent assumer de telles priorités s'éloignant parfois des standards du marché.

\section{Barrières internes}

Les barrières internes relèvent au niveau macroscopique de l'institution et s'expriment aussi au niveau microscopique chez les enseignants, personnels support (TIC) et étudiants. Les moyens humains (disponibilité des ressources et niveau de qualification) et financiers (coût d'acquisition et de développement de l'innovation), nécessaires au déploiement de nouvelles pratiques, représentent des freins importants (Corrocher et Fontana, 2008). L'instauration de certaines pratiques peuvent entraîner des coûts d'acquisition de nouvelles technologies, d'investissement dans des équipements spécifiques (tablettes, legos ${ }^{\circledR}$, salle de créativité, etc.), de formations des enseignants, voire d'une équipe d'animateurs, d'implications d'équipes techniques en support. À ce titre, des réticences au changement peuvent apparaître auprès des enseignants (Zwick, 2002).

La mise en œuvre d'outils d'apprentissage innovants implique effectivement de plus en plus le recours aux technologies - parfois nouvelles - et nécessite leur acceptation par les étudiants et les enseignants. Le niveau d'expertise technique et la maîtrise des technologies peuvent constituer des freins à leur mise en œuvre. Par ailleurs, la mise en œuvre de ces nouveaux dispositifs peut amener à repenser l'organisation des enseignements et à revoir des routines sécurisantes pour les personnels et étudiants. Les multiples missions de l'enseignant-chercheur laissent parfois peu de temps à l'innovation pédagogique, stimulante et gratifiante, mais dont l'investissement exigé est immense.

\section{Conclusion}

Si les sciences de gestion et du management sont des sciences de conception et de validation des modes d'action collective, si une théorie du management doit faire appel au concept de rhétorique, alors la mission de l'enseignement dans le champ est de proposer aux étudiants de nouvelles pratiques qui leur fournissent des compétences techniques et comportementales concrètes pour devenir manager. Le chapitre souligne la diversité des pratiques innovantes en milieu universitaire, ainsi que les principaux avantages et freins associés. La typologie proposée met en exergue l'usage croissant des technologies qui permettent le développement d'outils capables de simuler des environnements d'apprentissage riches et dynamiques. Par ailleurs, la ludification croissante des enseignements à l'université répond à une demande croissante d'aider les étudiants à maîtriser des compétences décisionnelles complexes. Enfin, l'évolution des pratiques d'évaluation pousse également les enseignants-chercheurs à proposer des pratiques innovantes afin de mesurer l'apprentissage basé sur les compétences. Les dispositifs de ludopédagogie sont de plus en plus nombreux dans les universités. Toutefois, à l'avenir, remplaceront-ils les traditionnelles études de cas dans les enseignements en sciences de gestion et du management? 

S'interroger 



\title{
Équité, justice organisationnelle et stress au travail
}

\author{
Le rôle du CNU \\ dans la carrière des enseignants-chercheurs
}

Hanane Beddi et Olivier Roques

Les enjeux d'augmentation du nombre d'étudiants, de professionnalisation des formations, d'embauche des diplômés et de production scientifique accentuent la nécessité pour l'université française d'avoir des personnels impliqués et motivés (Côme et Rouet, 2017). Pour ces auteurs, la clé d'un tel état d'esprit réside dans un système de gestion perçu comme " juste, équitable et transparent ". En revanche, si l'université ne sait pas fabriquer un tel système, le risque est grand de voir se développer du stress au travail, le manque de justice des systèmes constituant un risque psychosocial. Dans leur recherche, Nande et Commeiras (2020) ont interviewé un répondant, maître de conférences HDR, qui illustre bien ce propos : " Je pense qu'au niveau des concours et des avancements de carrière, c'est un truc, ils le feraient... au tirage au sort, cela serait exactement pareil à mes yeux... non, honnêtement, les systèmes d'évaluation sont aberrants ". Cette position, certes extrême, reflète toutefois la perception d'une partie des enseignants-chercheurs, qui ont, par ailleurs, développé plusieurs stratégies d'ajustement aux injonctions institutionnelles (Lussier et Chanlat, 2017). Certaines de ces stratégies sont " carriéristes » en considérant l'université sous un jour utilitariste. D'autres traduisent des comportements de repli et de résignation, témoignant d'un faible bien-être au travail, enfin d'autres acteurs se " sacrifient » en s'engageant dans le fonctionnement administratif de leurs institutions quitte à délaisser l'enseignement et la recherche.

L'objectif du chapitre est de proposer une réflexion autour des notions d'équité et de justice organisationnelle pour les enseignants-chercheurs universitaires. II interroge les systèmes de gestion de la carrière et met tout particulièrement en lumière le rôle du CNU. La première partie mobilise la théorie de l'équité, en examinant ses dimensions externe et interne, pour éclairer la question de la carrière des enseignants-chercheurs. La deuxième partie s'appuie sur les quatre dimensions de la 
justice organisationnelle pour mettre en évidence le rôle de gardien des procédures du CNU et son rôle d'information auprès des collègues. Enfin, la troisième partie se fonde sur le modèle de stress de Siegrist (1996). Celui-ci considère le déséquilibre effort-récompense comme déterminant de la qualité de vie au travail, du mal être au travail et du burnout.

\section{L'apport des recherches sur l'équité}

Parmi les théories psychologiques sur la motivation, Adams (1965) a introduit la théorie de l'équité qui vise à expliquer comment les salariés répondent à la perception d'injustice dans leur milieu de travail. Cette théorie insiste plus sur les données relatives (ratio d'inputs sur outputs) que sur les valeurs absolues. Plus précisément, les inputs peuvent être définis comme la perception par le salarié de sa contribution au travail et les outputs sont des éléments tels que les rémunérations intrinsèques du poste, les avantages en nature, le statut du poste, les symboles liés au statut. Cependant, la comparaison entre inputs (ce que l'individu apporte à l'organisation) et outputs (ce que l'individu en reçoit) ne peut pas être réalisée par l'individu de manière directe : chacun fait appel à un processus de comparaison sociale. La théorie affirme que les individus s'efforcent de maintenir le rapport entre leur contribution au travail (inputs) avec leur rétribution (outputs) de façon équivalente à celui des personnes auxquelles ils se comparent (les référents). En d'autres termes, les situations d'inéquité créent une motivation à rétablir l'équité.

Deux modalités de l'équité sont souvent distinguées : l'équité externe et l'équité interne. Pour les enseignants-chercheurs universitaires en sciences de gestion et du management, plusieurs environnements constituent l'externe : I'international, les autres professions (que celle d'enseignant-chercheur) au niveau national ou encore les business schools. L'équité interne, quant à elle, relève du traitement entre les enseignants-chercheurs universitaires en France.

\section{L'équité externe}

L'équité externe, fondée sur une comparaison internationale de la profession d'enseignant-chercheur dans différents pays, est particulièrement difficile à établir. En effet, les titres à travers les pays ne recouvrent pas exactement les mêmes activités. Toutefois, pour permettre un commencement de réflexion, le site Galaxie - qui gère le recrutement et la carrière des enseignants-chercheurs à l'université en France publie des équivalences des titres ${ }^{1}$. Au niveau local, ce sont les universités qui sont amenées à se positionner sur ces questions : en effet, les candidatures étrangères sont évaluées établissement par établissement, par le comité de sélection qui gère le recrutement, en nommant deux rapporteurs chargés d'évaluer la dispense de qualification du candidat, avec une validation ensuite du Conseil académique de l'établissement. Les comparaisons de traitement dans différents pays, à titre égal, sont un classique de l'activité de la GRH internationale (Cazal et al., 2010). Cela oblige à tenir compte de la rémunération liée à l'activité de base, mais aussi à la part de

1 Voir le lien Internet [https://www.galaxie.enseignementsup-recherche.gouv.fr/ensup/pdf/EC_pays_ etrangers/Tableau_comparaison_au_26_septembre_2012.pdf], consulté le 26 mai 2021. 
prélèvements sociaux et aux avantages sociaux du pays considéré (éducation, santé, infrastructures, vie culturelle, etc.). Le coût de la vie du pays considéré joue également pour établir l'équité externe (Cerdin, 2007).

De plus, l'équité externe est interrogée au niveau national, par rapport à d'autres métiers. Point et al. (2017) relatent une baisse des traitements réels nets de $16 \%$ pour les maîtres de conférences de seconde classe de 1981 à 2004 et constatent que cette baisse se poursuit. Là encore, les comparaisons sont complexes. Par exemple, dans le secteur privé, il est habituel que les salaires dépendent du lieu d'exercice du travail. Pour les enseignants-chercheurs, la réponse est apportée par l'indemnité de résidence. En pratique, celle-ci couvre très imparfaitement les différences de coût de la vie, notamment pour l'accès à la propriété. Complication supplémentaire, la rémunération des enseignants-chercheurs est faible au début de la carrière et croissante par la suite, avec des conditions de calcul de retraite plus favorables que dans le privé. Cela rend difficile la comparaison à un instant t puisque le système de rémunération des enseignants-chercheurs est conçu tout au long de la vie. Les avantages psychologiques d'un tel système de rémunération sur la fidélisation des collaborateurs sont souvent mis en avant, mais le rôle d'un étalement différé des paiements sur la trésorerie de l'employeur ne doit pas être négligé. De même, l'emploi à vie peut être conçu comme un avantage pour l'individu même s'il n'a pas de valeur monétaire. En pratique, il en a une pour l'employeur : les enseignants-chercheurs universitaires ne courant pas le risque de vivre des périodes de chômage, leur employeur s'exonère de ces cotisations.

La particularité des sciences de gestion et du management est de faire coexister un système universitaire et un système de business schools. Point et al. (2017) signalent d'importantes différences de salaire en faveur de ces dernières. Ainsi, pour les personnes en début de carrière, le salaire médian est $50 \%$ supérieur à celui de l'université. Pour les auteurs, ces différences au sein de la même profession risquent de conduire à un clivage entre enseignants-chercheurs :

D'un côté ceux qui travaillent en [business school], mieux payés, plus internationaux, plus mobiles et de l'autre ceux qui travaillent en université, moins bien payés (surtout en début de carrière), moins mobiles et très dépendants de leur production de recherche pour leur avancement.

II semble utile toutefois de nuancer ces propos au regard de l'actualité. En effet, des mesures ont été votées dans le cadre de la loi de programmation de la recherche (LPR), avec pour conséquence de rééquilibrer l'équité externe, en revalorisant le métier d'enseignant-chercheur universitaire comme, par exemple, le relèvement de la PRES (qui touche les maîtres de conférences et les professeurs des universités, la première catégorie plus que le seconde). Concernant les maîtres de conférences, le développement de postes contractuels à rémunération plus forte ou encore la revalorisation des nouveaux entrants (en termes de rémunération comme de conditions de travail [réduction des heures d'enseignement]) visent à rendre la profession plus attractive, moins inéquitable comparativement à d'autres professions. Toutefois, l'équité interne s'en trouve discutée. En effet, ces revalorisations peuvent entraîner un sentiment d'inéquité des plus anciens (moins bien rémunérés, par exemple) par rapport aux "contractuels ", et inversement un sentiment d'inéquité des nouveaux maîtres de conférences disposant d'un statut plus précaire que les anciens 
(fonctionnaires et quasiment impossibles à licencier). Ainsi, les nouvelles mesures permettent de répondre à un sentiment d'inéquité externe et, en cela, répondent à une tension, mais elles introduisent d'autres problèmes (les nouveaux recrutés sont mieux payés ou ont accès à des promotions plus rapidement que les plus anciens). C'est alors l'équité interne qui entre en jeu.

\section{L'équité interne}

Comme nous l'avons vu, les comparaisons externes sont complexes et difficiles à réaliser pour les salariés. En revanche, ces derniers disposent, en général, de plus d'informations relatives aux contributions et rétributions de leurs pairs. Cela rend leur sensibilité en termes d'équité interne plus forte qu'en matière d'équité externe. Cependant, du fait des situations contrastées selon les sections du CNU, il est difficile de dessiner les contours d'une équité interne dans le cas des enseignants-chercheurs universitaires. L'un des enjeux principaux est la capacité de ces derniers à être rémunérés de leur activité intellectuelle de manière libérale. Par exemple, les médecins peuvent avoir une activité privée qui complète leurs consultations publiques, et cela même dans les locaux de l'hôpital. Une telle tolérance vise à maintenir l'attractivité de l'hôpital public par rapport au privé pour les salariés. De même, les juristes peuvent mener une activité en dehors de l'université.

En revanche, les enseignants-chercheurs dans des domaines tels que l'histoire ou la philosophie ont plus de difficultés à faire valoir leurs compétences dans le privé. Quant aux enseignants-chercheurs de la section $06 \mathrm{du}$ CNU, ils ont la possibilité d'avoir une activité libérale. Cela peut être une activité de conseil, d'études ou de formation. Elle peut se diversifier aussi à travers des Diplômes Universitaires (DU) qui sont une source de financement pour les universités. Notons tout de même la difficulté à valoriser ces activités privées - cela nécessite des autorisations de cumul (de leur employeur principal), voire de passer par des organisations régies par le droit privé. Ainsi, l'hétérogénéité des enseignants-chercheurs rend difficile une réglementation homogène de la profession. Or, les CNU disciplinaires ont une bonne connaissance de l'écosystème dans lequel évoluent les membres de leur section et ils sont les plus à même de faire remonter au ministère la nécessité d'ajuster les conditions à l'intérieur des différentes sections.

\section{L'éclairage des théories sur la justice organisationnelle}

Après une première vision apportée par la théorie de l'équité à travers la comparaison entre contribution et rétribution, nous proposons d'enrichir notre réflexion grâce à la mobilisation du concept de justice organisationnelle (Greenberg, 1987). Plusieurs taxonomies de la justice organisationnelle cohabitent. La recherche de Colquitt (2001) permet, à travers un questionnaire largement diffusé, de lier concept et mesure, et ainsi de présenter quatre dimensions de la justice organisationnelle. Tout d'abord, nous présentons les deux premières dimensions, la justice distributive et la justice procédurale, qui sont les plus classiquement retenues par la littérature. Ensuite, nous évoquons les deux dimensions plus récentes, mais aussi plus controversées : la justice informationnelle et la justice interpersonnelle. 


\section{La justice distributive et la justice procédurale}

La justice distributive dérive directement de la notion d'équité d’Adams (1965). Elle pourrait se résumer par l'idée " à travail égal salaire égal ». La justice procédurale, quant à elle, concerne les règles de décisions relatives à l'attribution et à la répartition des outputs. Les travaux de Greenberg (1987) montrent bien que ces deux aspects de la justice ne coïncident pas forcément. Une même personne peut constater que les règles d'attribution des rétributions sont appliquées comme prévu avec l'organisation (justice procédurale), mais que des salariés recrutés à un autre moment ne reçoivent pas la même rétribution pour le même travail (justice distributive). Concernant les enseignants-chercheurs, le rôle du CNU prend tout son poids sur ces questions. En effet, en étudiant de nombreux dossiers en un temps réduit, notamment pour les qualifications, le CNU s'assure que les mêmes règles s'appliquent pour tous. II est fréquent, dans les débats, qu'une forme de jurisprudence s'instaure du type " vu que telle chose a été acceptée dans le cas $X$, alors il faut l'accorder dans le cas $Y$ ». Nous sommes bien face à un cas de justice procédurale. La dimension historique est en revanche plus difficile à assurer car les critères peuvent évoluer avec le temps (d'autant que le CNU se renouvèle tous les quatre ans). Comme toutes les autres organisations, sur la durée, le CNU peut espérer garantir une justice procédurale... beaucoup moins une justice distributive.

Selon Folger et Konovsky (1989), les individus sont affectés par la justice perçue des processus de prise de décisions indépendamment de la justice perçue de la décision elle-même. Plus précisément, les recherches sur l'influence de la justice procédurale et distributive sur le comportement des salariés montrent que les facteurs procéduraux déterminent l'implication dans l'organisation (Cropanzano et Folger, 1989), alors que les facteurs contribuant à la justice distributive déterminent une satisfaction plus spécifique, uniquement liée au salaire reçu. Une conséquence pratique est la distinction entre la motivation à agir de manière " constructive ", par exemple en redoublant d'efforts pour prouver que l'évaluation de ses résultats est injuste, et la motivation à agir de manière " destructive ", par exemple en travaillant moins ou en se mettant en grève, soit parce que l'évaluation est jugée inéquitable, soit parce qu'une distribution injuste des récompenses lèse directement les personnes concernées. Comment se fait le choix d'un comportement constructif ou destructif ? C'est l'équité perçue du processus qui joue un rôle décisif : si les procédures utilisées sont jugées équitables, il devient difficile de contester la répartition des récompenses qui en résulte. Par exemple, une évaluation qui semble être anormalement défavorable, mais qui résulte d'un processus considéré comme équitable, ne déclenchera pas une motivation à riposter contre la hiérarchie ou contre l'organisation. En revanche, si la procédure utilisée est vue comme injuste, la réaction sera destructive. Concernant les universités, de tels résultats se retrouvent chez Côme et Rouet (2017), qui mettent en évidence les débats pour l'octroi d'une prime dans diverses catégories de BIATSS et les vertus de la concertation et de l'explication du système. C'est bien sur les leviers de la justice procédurale qu'il est possible d'agir.

Les recherches montrent le rôle du CNU comme gardien de cette justice avec la mise en évidence de l'importance des critères sur le plan national. Ceux-ci doivent être collégialement discutés et validés au sein du CNU; ils doivent aussi être communiqués et expliqués au niveau de la communauté pour obtenir adhésion. La 
particularité des sections du CNU, par opposition aux instances locales, est d'assurer une justice procédurale, en quelque sorte " de branche ", alors que les instances locales mettent plutôt l'accent sur les particularismes du territoire. Cette double logique " branche " et " territoire " est habituelle, par exemple, dans l'organisation des syndicats. Son intérêt est sa capacité à analyser finement les enjeux. En effet, l'analyse au niveau des territoires (pour nous, au niveau des universités) sait prendre en compte l'environnement socio-économique, géographique, historique, culturel, artistique, etc. L'analyse en termes de branche (ici, de section CNU) sait prendre en compte des particularités de l'emploi (par exemple, pour la section 06 du CNU, la concurrence des écoles de commerce françaises, la concurrence de l'enseignement du management au niveau mondial, la possibilité de vendre des prestations intellectuelles de formation ou de conseil comme activité libérale). Ainsi, une instance nationale ne doit pas être vue comme un frein à la décision locale, mais plutôt comme un régulateur de l'action locale.

\section{La justice informationnelle et la justice interpersonnelle}

Nous mobilisons les deux autres dimensions de la justice organisationnelle de Colquitt (2001) : la justice informationnelle et la justice interpersonnelle. La justice informationnelle découle des éléments donnés pour expliquer les process et la façon d'obtenir les rétributions. La frontière entre justice interactionnelle et justice procédurale mérite une illustration. Ainsi, le document « Repères pour l'exercice du métier d'enseignant-chercheur » de $2019^{2}$ peut relever de la justice procédurale. II revêt un caractère officiel en se fondant sur un dialogue social avec les syndicats et en s'appuyant sur des textes. Toutefois, il est d'une maigre utilité pour comprendre comment réaliser un dossier de qualification, de Congé pour recherches ou conversions thématiques (CRCT), de prime d'encadrement doctoral et recherche (PEDR) ou de promotion. La justice informationnelle est donc relative à la capacité à avoir accès aux critères de prise de décision. L'étude empirique de Gatignol (2014) met en avant les difficultés à accéder à cette information : "Même s'il existe des règles officielles, il semblerait que les membres des commissions aient les leurs propres et que le fonctionnement de ces instances ne soit pas cohérent avec ce que disent les textes ".

Certains enseignants-chercheurs interviewés dans le cadre de la recherche de Nande et Commeiras (2020) vont dans le même sens, déclarant ne pas comprendre la logique des décisions. En complément, il est utile de mentionner que la section 06 du CNU a fait un effort de justice informationnelle à travers ses communications. Celles-ci se font au niveau de la présidence du CNU ou des prises de parole des différentes listes (comme, par exemple, la liste Variance avec son site Internet, qui se veut informatif). Différents comptes-rendus sont ainsi disponibles après les sessions du CNU pour informer les candidats des décisions prises, des modes de prise de décision, ce qui est également source d'informations et de conseils pour les futurs candidats aux différentes actions du CNU (demandes de qualification, de promotion, de CRCT, de PEDR). Cette approche a longtemps été peu développée mais il est - maintenant

2 Voir le lien Internet [https://cache.media.enseignementsup-recherche.gouv.fr/file/Personnels_ens._ sup_et_chercheurs/86/3/reperes_exercice_metier_enseignant_chercheur_1145863.pdf], consulté le 11 mars 2021. 
et depuis plusieurs années - considéré comme " normal » de communiquer. C'est un argument d'ailleurs mis en avant lors des élections du CNU. Cela concourt ainsi à la transparence des dispositifs.

La justice interpersonnelle, quant à elle, relève du respect et de la dignité avec lesquels sont traitées les personnes et, par extension, leurs dossiers, si l'on considère le cas des enseignants-chercheurs. Un tel élément souligne l'importance du CNU et de sa respectabilité. En effet, ses décisions peuvent être contestées en cas, par exemple, de favoritisme, de proximité (personnelle ou professionnelle) entre des candidats et des membres du CNU. Certaines règles ont d'ailleurs été instaurées : le directeur de thèse d'un candidat ( $s^{\prime} i l$ siège au $\mathrm{CNU}$ ) sort lors du traitement du dossier de ce dernier, les membres du CNU provenant de la même université qu'un candidat ne peuvent pas s'exprimer sur ce dernier, le choix des rapporteurs de ces dossiers tient également compte de ces éléments. Notons toutefois la difficulté d'avoir connaissance des éventuels liens existants, une asymétrie d'information perdurant en dépit des dispositifs mis en place. Enfin, la question du traitement des membres du CNU eux-mêmes, et du signal que cela envoie, peut être posée, à travers le principe de l'auto-promotion qui fait débat chez les enseignants-chercheurs.

\section{La mobilisation des approches liées au stress à travers le modèle de déséquilibre effort-récompense}

Si le cadre conceptuel de l'équité et de la justice est souvent rattaché aux théories de la motivation, le modèle de déséquilibre effort-récompense (effort-reward imbalance, ou ERI) de Siegrist (1996) donne des clés pour expliquer le stress au travail. II alimente le débat sur les risques psychosociaux et la qualité de vie au travail.

\section{Le modèle de déséquilibre}

Pour Siegrist (1996), le modèle de déséquilibre effort-récompense est une contribution aux théories du stress (figure 1). Siegrist revendique une filiation avec l'approche " job demand-control » de Karasek (1979) qui, pour déterminer la charge mentale (strain) du salarié, identifie deux dimensions : les exigences du travail (job demand) et la latitude décisionnelle (job control). Plus précisément, la récompense attendue en échange des efforts déployés au travail peut prendre plusieurs formes, aussi bien matérielle (rémunération) qu'en termes de reconnaissance et de perspectives professionnelles (promotion, sécurité de l'emploi). Or, " selon ce modèle, l'absence de réciprocité (effort intense combiné à faible récompense) est un phénomène fréquent et engendre des émotions négatives fortes et des réponses au stress qui ont à long terme des effets néfastes sur la santé » (Siegrist, 2012). 

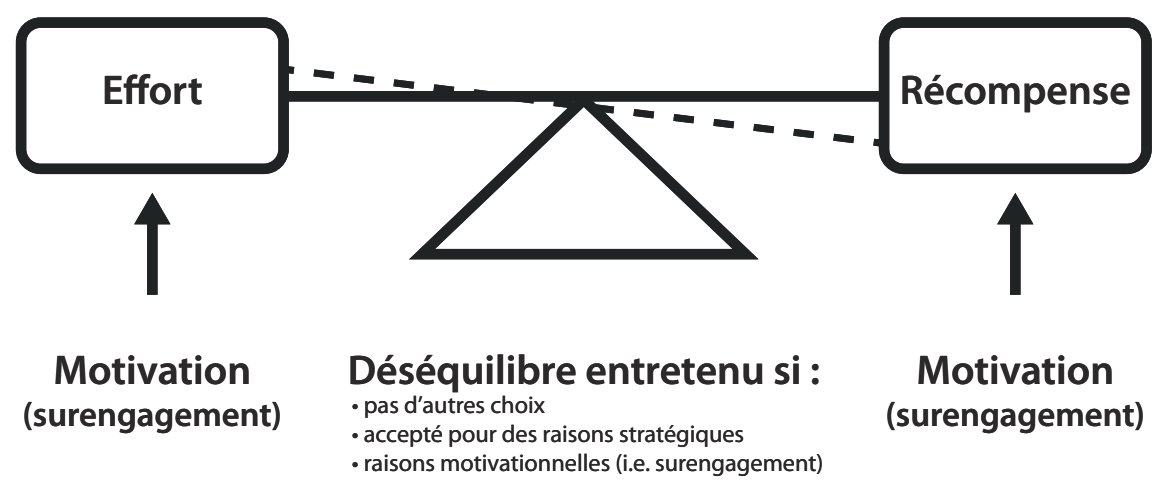

Figure 1 : Le modèle de déséquilibre effort-récompense Source : d'après Siegrist (1996)

En pratique, le déséquilibre effort-récompense prend la forme d'un ratio avec les efforts au numérateur et les récompenses au dénominateur. Les précédents modèles de stress, comme celui de Lazarus et Folkman (1984), voient le stress comme la conséquence d'une mise à l'épreuve ou d'un dépassement des capacités de l'individu. $A$ contrario, le modèle de stress de Siegrist (1996) défend l'idée que les récompenses fournies par l'organisation peuvent éviter le burnout. Ainsi, selon Siegrist (2012), un très haut niveau d'activité professionnelle (les " efforts ") n'est pas un problème en soi s'il est justement reconnu par l'organisation (les « récompenses ») : c'est pour lui une forme de "contrat social ". Le manquement à cette réciprocité conduit à des manifestions de stress. De plus, Siegrist et Wahrendorf (2016) ont toujours veillé à mixer des mesures d'attitude et de comportement avec des mesures médicales, qui se trouvent vérifiées par de nombreux travaux internationaux rassemblés dans leur ouvrage collectif.

Par transposition, la reconnaissance des " efforts " des enseignants-chercheurs à travers les promotions, les PEDR ou la qualification est au cœur de l'activité de la section 06 du CNU. Selon nous, elle prend la forme d'une responsabilité sociale envers les enseignants-chercheurs pour leur éviter mal-être au travail et burnout. Au niveau local, une telle reconnaissance est également de mise, au sein des conseils d'établissement, qui peuvent en tenir compte, pour l'octroi de promotions locales, ou même dans les décisions de recrutement spécifique comme la « voie longue » des professeurs des universités (article 46.3).

\section{La dynamique du surengagement}

L’approche de Siegrist (2012) ne se limite pas à décrire l'existence d'un déséquilibre; elle définit des conditions qui conduisent au maintien de cet état. Lorsque l'individu est fortement impliqué dans son activité du fait d'une motivation intrinsèque, mais qu'il constate que l'organisation ne le récompense pas de ses efforts, il est dans une situation de surengagement. Les efforts dépassent les récompenses et la personne évolue vers des manifestations de stress ou de burnout. Ces éléments font écho à la recherche de Côme et Rouet (2017) qui, en s'appuyant sur l'enquête 
EducPros réalisée en 2016, atteste d'un tel cisaillement entre motivation intrinsèque et manque de reconnaissance extrinsèque :

Si les professionnels de l'enseignement supérieur restent " passionnés " d'être au service des étudiants et de la recherche, le manque de reconnaissance, symbolique comme financier, explique la démotivation déclarée par $47 \%$ des sondés volontaires.

Plus précisément, Siegrist (2012) identifie trois situations qui encouragent le maintien dans la situation de déséquilibre :

- Une absence de choix professionnel pour s'échapper de la situation de déséquilibre. Cela peut venir d'un manque d'employabilité, ou d'un manque de postes. C'est le cas des enseignants chercheurs de la section 06 confrontés à la compétition interne pour les postes de professeur des universités, qui représentent seulement $25 \%$ des postes d'enseignant-chercheur (soit l'un des plus bas de la profession). De plus, les évolutions peuvent être conditionnées à des mobilités géographiques pour retrouver des conditions d'emploi plus favorables. Si, pour accéder à un changement de grade, ils doivent déménager ou financer des déplacements, le peu d'intérêt de la nouvelle situation peut les conduire à entretenir une situation de déséquilibre entre leurs efforts et les récompenses organisationnelles qui leur sont par ailleurs refusées dans leurs institutions d'origine. Cela peut alors entraîner une évolution vers des manifestations de stress.

- Le déséquilibre peut être accepté pour des raisons stratégiques. Conçus comme des investissements, les efforts déployés par anticipation visent à assurer une position plus favorable dans le futur. Bien sûr, l'individu attend une réponse de l'organisation. Cette reconnaissance, dans le cas d'un enseignant-chercheur, est attendue localement, par son institution d'origine. En complément, le CNU a aussi un rôle à jouer dans la définition de critères nationaux, pour arriver à une reconnaissance d'un tel investissement.

- Enfin, des raisons motivationnelles et cognitives propres à l'individu peuvent le pousser à des degrés d'engagement excessifs par rapport à ses collègues : ne pas savoir dire non pour le suivi d'un projet, se dévouer pour le maintien à flot d'une institution, etc. Lussier et Chanlat (2017) attribuent ce comportement à un " ethos professoral » qui repose sur le fait de bien faire son travail indépendamment des récompenses offertes par l'organisation. Les auteurs soulignent cependant les risques pour une institution à compter sur cette forme d'altruisme par ailleurs de plus en plus rare. Selon nous, cette situation peut d'ailleurs tenir à court terme mais, dans le temps, le risque est que l'individu décide d'arrêter de s'impliquer totalement. Ainsi, il n'est pas rare d'avoir, dans nos établissements, des personnes surengagées et des personnes faiblement engagées (mais après avoir été surengagées des années durant sans contrepartie institutionnelle).

Le rôle du CNU est justement de veiller à la juste récompense des actions menées par les enseignants-chercheurs. Il se pose (ou devrait se poser) clairement comme le gardien pour éviter toutes dérives au niveau local. Ainsi, il peut permettre de contrebalancer les déséquilibres préalablement décrits, en objectivant au maximum ses décisions dans le cadre de ses missions et en ayant conscience des risques sur le stress des individus, les risques psychosociaux et la qualité de vie au travail. 


\section{Conclusion}

Les théories mobilisées dans le présent chapitre permettent de questionner le rôle du CNU, tel que perçu ou attendu par les enseignants-chercheurs. Le CNU œuvre dans un contexte complexe, au niveau international (compétition accrue, difficulté de comparaison) et national (loi LPR), et il doit veiller aux différentes formes d'équité. En matière de justice, il semble utile d'œuvrer à la clarification des process en proposant une information plus transparente. La sensibilisation au stress, notamment au surengagement, est également essentielle. Ainsi, le CNU se pose - ou devrait se poser - comme le garant, le gardien ou le régulateur de l'équité et la justice. Le lien avec la responsabilité sociale envers les enseignants-chercheurs est une piste pertinente à développer.

Si les théories sur l'équité, la justice organisationnelle ou le stress au travail sont classiques et relativement anciennes, elles trouvent cependant une grande actualité et utilité lorsqu'on les mobilise pour éclairer les enjeux de carrière des enseignants-chercheurs, tout particulièrement en les croisant avec les recherches qui se développent sur ces derniers. Enfin, pour conclure, le rôle du CNU n'est pas aisé à tenir, notamment vis-à-vis des institutions dans lesquelles opèrent les enseignants-chercheurs. Notre chapitre défend l'idée d'une logique de complémentarité et non d'opposition qui doit (ou devrait) œuvrer entre la voix nationale et la voix locale. À l'instar du fameux adage marketing " think global, act local ", l'idée défendue ici est qu'une instance nationale n'exclut pas la liberté locale. 


\section{Et si on parlait de l'attractivité du métier d'enseignant-chercheur en gestion?}

Thérèse Albertini, Delphine Bereni et Solange Hernandez

À la question "Que veux-tu faire plus tard? ", les enfants répondent rarement universitaire ou enseignant-chercheur. Ils aspirent davantage à devenir docteur (en médecine), maître d'école (pour transmettre), pompier (pour sauver des vies), coiffeur (pour embellir des vies), fleuriste (pour apporter des sourires), astronaute (pour découvrir de nouveaux horizons), scientifique (pour expérimenter), ingénieur (pour inventer), avocat (pour défendre des causes), écrivain (pour poser ses idées), chanteur (pour faire entendre ses idées), pilote (pour être à la manœuvre), etc. Transmettre, inventer, découvrir, démontrer, défendre, écrire, exposer, sauver, embellir, voilà autant de facettes de notre travail d'universitaire ! Pourtant, les enseignants-chercheurs, titulaires de l'enseignement supérieur de l'Université française, à savoir les professeurs des universités et maîtres de conférences exercent un métier bien peu compris, alors même qu'il est riche, stimulant et engageant.

Notre attention portera spécifiquement dans le présent chapitre sur les enseignants-chercheurs en sciences de gestion et du management (dénommés ECG par la suite), même si, probablement, nombre de collègues issus de disciplines différentes se reconnaitront certainement ici, notamment dans la description et la pluralité des activités décrites, ainsi que dans les satisfactions et difficultés liées à l'exercice de la fonction. Notre volonté est de mieux faire connaître le métier " mystérieux " d’ECG et d'en apprécier l'attractivité. Dans cette perspective, nous étudierons successivement les spécificités du métier, ses avantages et ses tensions sous-jacentes. Enfin, nous porterons notre attention sur des pistes d'amélioration, tenant compte des principes fondateurs du métier.

\section{Plantons le décor}

Qu'est-ce qu'un enseignant-chercheur en sciences de gestion et du management? La première partie pose le cadre juridique qui régit cette profession, précise quels 
sont les objets des enseignements et des recherches, puis fournit des chiffres clés permettant de mesurer les charges et responsabilités des ECG.

\section{Cadre juridique du métier d'enseignant-chercheur}

La profession des universitaires est longtemps restée sans statut, alors que le système universitaire français actuel se met en place à partir du $\mathrm{XIX}^{\mathrm{e}}$ siècle. Cela pourrait traduire le peu d'intérêt de l'État pour cette profession (Beaud et Vatin, 2020). Juridiquement, trois textes fondent le statut des universitaires : la loi Faure (loi $n^{\circ} 68-978$ du 12 novembre 1968 d'orientation de l'enseignement supérieur), la loi Savary (loi n 84-52 du 26 janvier 1984 sur l'enseignement supérieur) et le décret Pécresse (décret n²009-460 du 23 avril 2009 modifiant le décret n84-431 du 6 juin 1984 fixant les dispositions statutaires communes applicables aux enseignants-chercheurs et portant statut particulier du corps des professeurs des universités et du corps des maîtres de conférences et portant diverses dispositions relatives aux enseignants-chercheurs). Avant 1968, les enseignants des universités étaient fonctionnaires d'État. À ce titre, ils accomplissaient une mission de service public d'intérêt général : la formation au savoir par le savoir. La recherche n'était pas mentionnée. À partir de 1968, ils doivent exercer des "fonctions d'enseignement et des activités de recherche ".

En 1984, les maîtres de conférences et les professeurs des universités deviennent des enseignants-chercheurs, accomplissant, certes, deux fonctions, mais surtout en charge d'une liste d'activités " à la Prévert " : élaboration et transmission par l'enseignement des connaissances ; direction, conseil, tutorat et orientation ; développement de la recherche fondamentale, appliquée, pédagogique ou technologique; valorisation de ses résultats ; développement scientifique et technologique ; coopération entre la recherche universitaire, la recherche industrielle et l'ensemble des secteurs de production; diffusion de la culture et de l'information scientifique et technique; etc. L'enseignant-chercheur est ainsi en charge d'activités dévolues jusque-là à divers métiers dans l'université (enseignant, chercheur, chargé d'orientation, ingénieur de recherche, ingénieur administratif, secrétaire, etc.) (Sacriste, 2014). Son service, le nombre d'heures de cours à délivrer, passe de 128 à $192 \mathrm{~h}$ par an. Ces changements majeurs visent à faire face à la massification des étudiants dans un contexte émergent d'austérité budgétaire.

En 2009, la liste des activités des enseignants-chercheurs s'étend, encore. L'insertion professionnelle des étudiants, le management des formations, facultés et laboratoires sont désormais concernés. Bien que lesdites activités ne soient pas spécifiées (donc non rémunérées), l'enseignant-chercheur est désormais aussi manager et participe à la nouvelle gouvernance des universités (loi n 2007-1199 du 10 août 2007 relative aux libertés et responsabilités des universités). Ces fonctions additionnelles ne sont d'ailleurs pas intégrées au temps de travail car celui-ci est divisé équitablement (dans les textes) entre enseignement et recherche. Les enseignants-chercheurs sont donc $50 \%$ enseignant, $50 \%$ chercheur, et (50\% ?) manager. Les décideurs auraient-ils quelques lacunes en statistiques élémentaires? Un tel cadre législatif, sa traduction pour le quotidien et la carrière des enseignants-chercheurs, restent largement méconnus, alimentant de nombreuses idées reçues, comme nous le verrons par la suite. 


\section{Gestion, vous avez dit gestion?}

La gestion s'intéresse aux organisations (quel que soit leur statut, public, privé, associatif, etc.), et aux individus qui agissent - individuellement ou collectivement dans celles-ci ou en interaction avec celles-ci. Dans cette perspective, les sciences de gestion et du management appartiennent aux sciences sociales. On parlera de la science économique ou de la science politique mais on parlera des sciences de gestion et du management. Le lecteur notera l'usage du pluriel. Celui-ci reflète la richesse de cette discipline qui permet de cerner l'objet mystérieux que sont les organisations. Ainsi, les ECG développent leurs compétences en stratégie, marketing, management public, finance, GRH, logistique, systèmes d'information, entreprenariat, ou encore comptabilité. Une telle diversité offre une multitude de combinaisons possibles (en bleu dans la figure 1), et leur nombre s'accroît quand on y ajoute la diversité des champs d'application (en vert dans la figure 1) et des thématiques d'enseignement et de recherche (en mauve dans la figure 1). Car tout se gère ! Ces éléments sont déclinables en thèmes de recherche et en pédagogie.
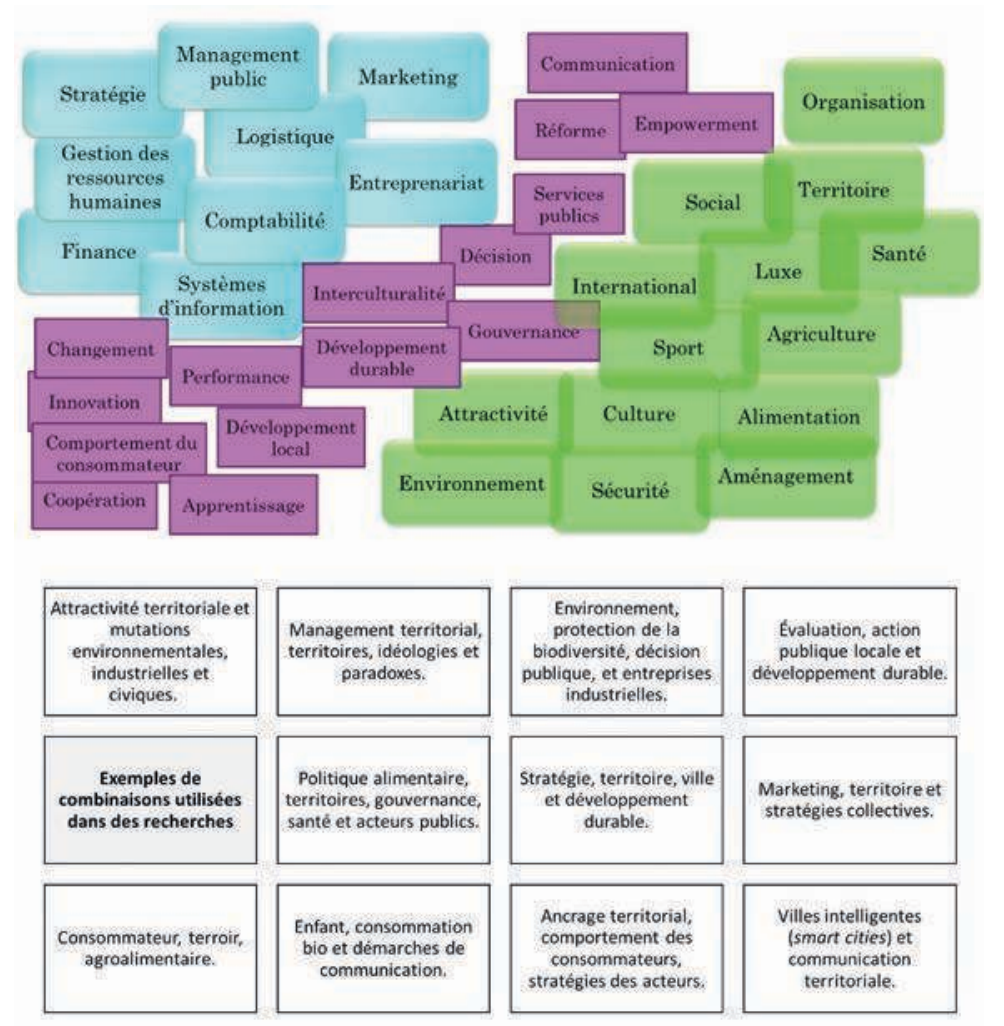

Figure 1 : La recherche en sciences de gestion et du management : une large variété de sous-disciplines, de secteurs et de thèmes Source : élaboration personnelle des auteurs 


\section{Quelques chiffes pour prendre la mesure}

Voici à présent des données permettant de mesurer à la fois les effectifs étudiants et ceux des ECG par rapport à l'ensemble des disciplines et de l'université. D'après le ministère de l'Enseignement supérieur, de la Recherche et de I'Innovation, en 2019-2020 ${ }^{1}$, les sciences économiques et de gestion (indissociées dans les statistiques) comptent 204838 étudiants (138 358 en licence, 63592 en master et 2888 en doctorat) sur un total de 1401241 étudiants (hors santé), soit 14,6 \%. Parmi eux, 40177 sont des nouveaux entrants, soit $12,4 \%$ de nouveaux étudiants. Pour une vue complète des effectifs en sciences de gestion et du management, il faut également ajouter ceux inscrits dans les 334 écoles de commerce, gestion et vente, soit 199200 étudiants.

Quant aux enseignants-chercheurs ${ }^{2}$, l'université française en compte 55538 (maîtres de conférences et professeurs des universités, titulaires et stagiaires). Dans les données du ministère ${ }^{3}$, ceux attachés aux sciences économiques et de gestion sont 3 881. Ces deux disciplines représentent donc respectivement 6,6 \% des enseignants-chercheurs de l'université française et ils ont la responsabilité de former près de $15 \%$ des étudiants. C'est un premier déséquilibre à noter. Intéressons-nous maintenant aux effectifs des enseignants en sciences de gestion et du management uniquement (contrairement aux étudiants, ils sont disponibles ${ }^{4}$ ), qui comptent 2143 personnes réparties en 541 professeurs des universités et 1629 maîtres de conférences. Ensuite, comparons ces chiffres avec ceux de l'ensemble. En moyenne, toutes disciplines confondues, les professeurs des universités représentent $32 \%$ des enseignants-chercheurs. En sciences de gestion et du management, ils sont seulement $24 \%$. C'est un second déséquilibre à observer.

En résumé, le sous-encadrement en sciences de gestion et du management est patent, et partout ! $7 \%$ des enseignants-chercheurs de l'université forment près de $15 \%$ des effectifs, et seuls $24 \%$ sont professeurs des universités (un quart contre un tiers en moyenne). Dit autrement, le ratio enseignants-chercheurs / étudiants est de 1 pour 53 en économie et gestion, contre 1 pour 25 en moyenne dans l'université. Chaque enseignant-chercheur en économie et gestion a en charge le double d'étudiants que la moyenne.

1 Repères et références statistiques sur les enseignements, la formation et la recherche, édition 2020 [https://www.enseignementsup-recherche.gouv.fr/pid24831-cid153537/reperes-et-references-statistiques-edition-aout-2020.html], consulté le 26 mai 2021.

2 Les personnels enseignants de l'enseignement supérieur se répartissent en trois grandes catégories : outre les 55538 enseignants-chercheurs, ils intègrent 13100 enseignants du second degré affectés dans l'enseignement supérieur et 22100 enseignants non permanents (dont la moitié de doctorants contractuels effectuant un service d'enseignement et des ATER).

3 Note de la DGRH-Enseignement supérieur, n 8, novembre 2020.

4 Fiches démographiques des sections du CNU, année 2019, section 06 [https://www.enseignementsup-recherche.gouv.fr/cid153532/fiches-demographiques-des-sections-de-droit-2019.html], consulté le 13 juin 2021. 


\section{Un métier formidable}

Le métier, même s'il est l'objet d'idées reçues, procure de nombreux avantages, offre des libertés, crée des émotions, enfin, il est à l'origine de nombreuses utilités sociales.

\section{Des avantages et des idées reçues}

Le métier d'enseignants-chercheurs, a fortiori celui d'ECG, est un métier sans limite spatio-temporelle. Nous travaillons partout, à l'université, au domicile, sur des campus extérieurs, dans les transports en commun, au restaurant, en présentiel, en distanciel. II est difficile de décrocher véritablement de nos activités. Pourtant, le métier fait face à bien des préjugés, dont beaucoup portent sur le temps de travail supposé (figure 2).

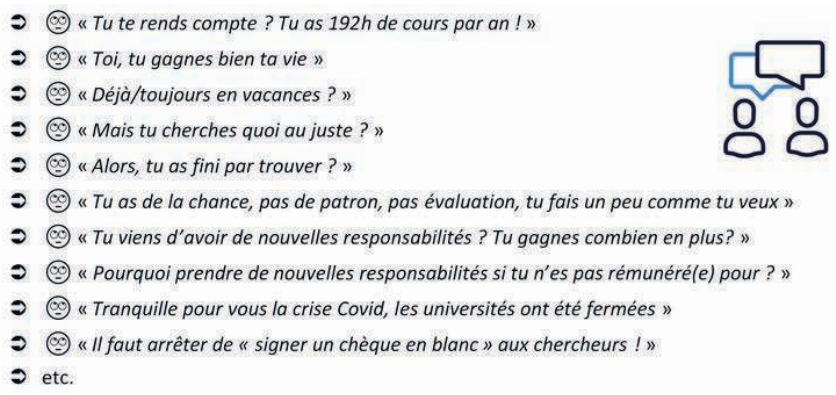

Figure 2 : Quelques préjugés sur le métier d'enseignant-chercheur Source : élaboration personnelle des auteurs

La liste est non exhaustive. Sans y répondre, signalons qu'au-delà des 192 h équivalent TD annuelles, s'ajoutent toutes les activités invisibles en matière d'enseignement (préparation, examens, corrections, jurys, recrutements, entretiens, réunions, encadrements, projets, suivis, etc.), de recherche (publications, appels à projet ou $\mathrm{AAP}$, évaluations d'articles ou de communications, rapports autorisant la soutenance de thèses, directions de thèses, comités de suivi, comités de sélection, colloques, etc.) et administratives (responsabilité de formation, direction de composante, conseils d'instance, etc.). En réalité, la diversité des activités exercées rend le métier intéressant et peu ordinaire. L'universitaire étant enseignant, chercheur et administratif, ses aptitudes, compétences et appétences sont sans cesse renouvelées. Entourés de collègues aux profils et personnalités diverses, nous évoluons dans une communauté de travail dynamique.

Au service de ses étudiants, l'organisation universitaire trouve ses fondements dans les valeurs de tolérance, de respect mutuel et de service. II paraît alors pertinent de dépasser les idées préconçues liées à la fonction d'enseignant-chercheur en présentant ses réels avantages qui permettent épanouissement intellectuel, personnel et humain (tableau 1). Cette conjugaison de satisfactions se retrouve, à titre d'illustration, dans l'affiche pour le recrutement en Master recherche, étude et conseil en gestion / management public de I'IMPGT d'Aix-Marseille Université (figure 3). 
Thérèse Albertini, Delphine Bereni et Solange Hernandez

Tableau 1 : Des avantages du métier à l'épanouissement de l'enseignant-chercheur

\begin{tabular}{|c|c|}
\hline Lire, écrire, réfléchir & \multirow{5}{*}{ Une stimulation intellectuelle continue } \\
\hline $\begin{array}{l}\text { Satisfaire sa curiosité, expliquer, échanger } \\
\text { Rompre avec l'ennui, diversifier ses réflexions }\end{array}$ & \\
\hline Se former continuellement, tout au long de la vie & \\
\hline Renouveler ses activités & \\
\hline Redécouvrir continuellement son métier & \\
\hline Organiser son temps de travail & \multirow{2}{*}{$\begin{array}{l}\text { Une harmonisation entre vie professionnelle } \\
\text { et personnelle }\end{array}$} \\
\hline $\begin{array}{r}\text { Lier ses thématiques d'études à ses passions, } \\
\text { son vécu }\end{array}$ & \\
\hline Transmettre, recevoir & \multirow{6}{*}{ Une riche aventure humaine } \\
\hline Voyager, découvrir & \\
\hline S'adapter, rebondir, s'affirmer & \\
\hline $\begin{array}{l}\text { Se surpasser, se surprendre } \\
\text { Faire preuve d'empathie, comprendre la jeunesse }\end{array}$ & \\
\hline $\begin{array}{r}\text { Comprendre le collectif, choisir ses collègues, } \\
\text { en faire des amis }\end{array}$ & \\
\hline $\begin{array}{r}\text { Rencontrer, intégrer une communauté, } \\
\text { créer des liens }\end{array}$ & \\
\hline
\end{tabular}

Source : élaboration personnelle des auteurs

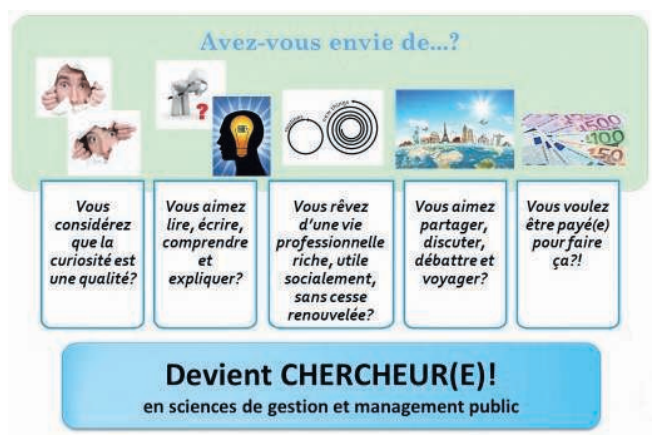

Figure 3 : Affiche de recrutement en Master recherche, étude et conseil en gestion / management public de I'IMPGT Source : création de S. Hernandez (2019-2020)

\section{Liberté, liberté chérie!}

Les universitaires n'ont pas d'obligation de présence régulière sur un lieu de travail ; leur seul temps contrôlé est la présence devant les étudiants (Beaud et Vatin, 2021). Au-delà de cette liberté organisationnelle clairement perçue par la société, l'ECG dispose d'une liberté intellectuelle appréciable (figure 4). Une telle latitude permet à I'ECG d'opter pour les activités qui l'intéressent, lui procurent du plaisir et génèrent une certaine satisfaction. Parallèlement, l'universitaire apprécie volontiers les défis, 
ce qui participe à approfondir et diversifier compétences et capacités tout au long de la vie professionnelle. Cet état d'esprit nourrit la motivation intrinsèque, se traduisant par un sentiment d'accomplissement.

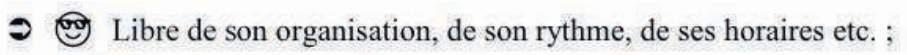

? . Libre de mettre en place son dispositif pédagogique,

? 2 Libre d'écrire un article, de le soumettre ;

• recherche, son terrain ;

? 응 Libre de participer à des conférences, congrès et autres manifestations académiques ;

$\Rightarrow$ etc.

Figure 4: Une liberté organisationnelle et intellectuelle (non exhaustif) Source : élaboration personnelle des auteurs

Cela renvoie à la théorie du flow, qui conduit à une absorption totale de l'individu (ici l'ECG) par son projet. Csikszentmihalyi (1990) met en relation neuf états psychologiques : le flow, la maîtrise, l'ennui, le détachement, l'indifférence, l'inquiétude, l'anxiété, l'excitation et un état neutre. Le flow correspond à l'état émotionnel caractérisé par un sentiment de concentration énergique, de pleine implication et de plaisir. Une telle sensation est éprouvée lorsque nous sommes pleinement concentrés à une activité plaisante, valorisante, dans laquelle tous les talents sont déployés. La mobilisation des ressources de l'ECG, la volonté individuelle et collective de s'engager dans la démarche, la liberté associée à ce choix, sont autant d'éléments qui maximisent les efforts et transforment l'accomplissement de la tâche en une expérience riche de sens (figure 5). Au regard de la liberté acquise par l'ECG et des défis auquel il se soumet régulièrement, l'expérience optimale décrite par Csikszentmihalyi (1990) est alors atteignable. Ce chemin vers l'expérience optimale est jalonné d'émotions stimulantes qui renforcent l'attachement au métier.

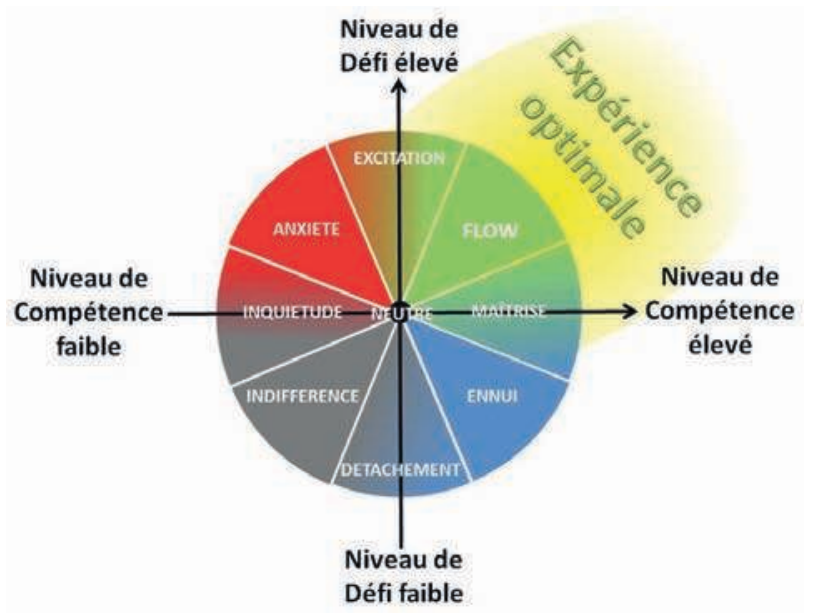

Figure 5 : La théorie du flow : le bonheur au travail Source : d'après Csikszentmihalyi (1990) 


\section{Des émotions fortes au quotidien}

La projection vers une expérience optimale déclenche des émotions. L'ECG doit sans cesse faire preuve de créativité, quitter sa zone de confort, se surpasser, ce qui crée de la satisfaction. Décider librement de relever un défi, se faire confiance, s'impliquer, se traduit par une récompense émotionnelle forte. Le métier d'ECG amène chacun à se redécouvrir continuellement, à prendre conscience de ses aptitudes, compétences et qualités souvent insoupçonnées (persévérance, estime de soi, niveau d'engagement, performance, créativité, etc.). Toutes ces émotions contribuent à l'épanouissement et l'évolution de l'ECG. II serait utopique de lister les expériences émotionnelles associées à la fonction, mais il est certain que ce métier est une réelle aventure humaine!

Audrin (2020) souligne que les interactions avec différents types de personnes (collègues, communautés académiques et scientifiques, étudiants) sont une source d'émotions fortes. Les interactions avec les étudiants sont citées comme celles induisant les émotions plus intenses. La satisfaction ressentie lorsqu'on les accompagne, constate leur progression, et lorsque ces derniers nous témoignent leur reconnaissance, nous amène à savoir " pourquoi on se lève ". Ces témoignages de reconnaissance sont souvent informels mais révélateurs de notre rôle social. D'anciens étudiants deviennent ambassadeurs des formations, s'impliquent dans la vie de l'établissement (accueil de stagiaires, d'apprentis). Ils témoignent de leur insertion professionnelle en l'associant aux enseignements reçus; en atteste l'essor sur Linkedln des relations anciens étudiants / enseignants avec un suivi post-étude du devenir des apprenants. Parfois, cette gratitude se formalise par des attentions, des confidences, des appels à l'aide, un besoin de partager les bonnes nouvelles. De telles relations de proximité coconstruites font " chaud au cœur » et encouragent à poursuivre nos efforts et notre engagement auprès des étudiants.

Les émotions interactionnelles se retrouvent également dans les liens tissés avec les collègues, et ceci malgré une concurrence accrue entre eux. Il y a bien évidemment les collègues de fait et les collègues choisis, avec qui l'on décide librement de collaborer dans la co-écriture d'articles, les projets de recherche, l'animation d'ateliers dans un congrès, un jury ou une codirection de thèse. Autant d'éléments qui génèrent un partage quotidien, un échange continu et fertile... jusqu'à devenir parfois des amis. Les " collègamis " sont une richesse du métier, permise par notre liberté de choisir nos partenaires et nos modes de travail. Ces relations, professionnelles et amicales, se traduisent, par exemple, par la corédaction d'un chapitre d'ouvrage sur l'attractivité de notre métier d'enseignants-chercheurs! Les travaux partagés sont soumis à la considération des collègues à travers des procédés d'évaluation. La reconnaissance par les pairs est l'une des incitations fortes dans le métier d'ECG. Les échanges constructifs lors des congrès, colloques, séminaires permettent à l'ECG d'apprécier l'acceptabilité et la qualité du travail de recherche et le font évoluer. Ainsi, l'acceptation d'un article dans une revue reconnue est un moment d'euphorie et d'accomplissement, renforçant le chemin vers l'état de flow évoqué antérieurement. Le métier d'ECG est donc source d'émotions individuelles et collectives, intimement liées aux utilités sociales et sociétales de la fonction universitaire. 


\section{De l'utilité sociale et sociétale de l'enseignant-chercheur}

Enseignant-chercheur est à la fois un métier, l'accomplissement d'un rôle institutionnel et la mise en œuvre d'une personnalité (Dubet, 2020). L'utilité sociale du métier est communément reconnue. L'ECG prend plaisir à organiser ses idées et transmettre son savoir. II a des motivations altruistes et collectives qui s'articulent essentiellement autour de la volonté de participer à l'avenir de la jeunesse, d'améliorer l'équité sociale, de réaliser une contribution sociétale tout en travaillant auprès d'un public en quête de connaissances, d'échanges et d'interactions (Berger et D'Ascoli, 2011).

La motivation des ECG est certes impulsée par la formation des futurs managers, mais celle des citoyens éclairés de demain (développement de l'esprit critique, capacité à prendre du recul, à s'interroger, prise de conscience des responsabilités sociales, environnementales et sociétales, etc.). Déclarer l'utilité sociale et sociétale de ce métier est une forme de reconnaissance, conduisant vers " l'expérience optimale » (figure 5). Conscients du grand courage d'étudiants, pour qui la vie n'est pas toujours un long fleuve tranquille, les ECG, forts de leur engagement, s'emparent des questions relatives aux problèmes de société ou d'organisation et s'efforcent de les aider à s'accomplir comme professionnels et citoyens.

La crise sanitaire et économique générée par la pandémie de Covid-19 a nécessairement affecté les perceptions utilitaires des professions. Le travail des ECG a été bouleversé par un fonctionnement 100 \% numérique, peu propice aux échanges universitaires. Ceux-ci ont alors développé des pratiques pédagogiques innovantes, humanisant autant que possible le distanciel, favorisant les apprentissages et maintenant la dynamique des promotions étudiantes. Même si cette période a constitué une fracture, de nombreux étudiants ont développé des compétences spécifiques, gagné en autonomie, en prise d'initiatives et en organisation de travail. En bref, il est primordial que la formation de la jeunesse demeure qualitative, interactive, dynamique et garante de la démocratisation des études supérieures en France (ascenseur social). Cependant, même si l'exercice du métier d'enseignant-chercheur s'avère passionnant, il n'est pas si aisé, comme en attestent les tensions inhérentes au métier.

\section{Un métier sous tension}

Accéder au métier d’ECG est un parcours long, difficile, sélectif, au cours duquel chacun apprend beaucoup sur lui-même et ses capacités de travail et de réflexion. Au quotidien, exercer les missions dévolues fragilisent de plus en plus la sphère privée. La crise sanitaire a largement accentué la tendance, mais n'en est pas à l'origine. Il y a toujours un papier à valoriser, un cours à actualiser, une nouvelle étude de cas à produire, des courriels en attente, une réunion à préparer, des candidatures à étudier, des copies à corriger, un colloque à organiser, des mémoires ou des thèses à encadrer, à évaluer, une participation à un jury, un montage de projet, des financements à trouver, etc. Ces activités chronophages occasionnent des tensions, voire des souffrances. Sans prétendre à l'exhaustivité, nous portons notre attention sur quatre niveaux de tension, souvent exprimées par les ECG : (1) le stress et les frustrations occasionnées par l'exercice de ce métier aux multiples facettes; 
(2) l'évaluation continue par les pairs ; (3) les aspects financiers ; et (4) la pression en faveur de l'usage de l'anglais.

\section{Un métier tridimensionnel stressant et souvent frustrant}

Être enseignant-chercheur, c'est exercer plusieurs métiers en un (Beaud et Vatin, 2021), chacun ne mobilisant pas les mêmes compétences et connaissances. Outre l'enseignement et la recherche, l'enseignant-chercheur endosse le rôle de manager, consistant à exercer diverses activités administratives et institutionnelles (responsable pédagogique, référent d'une unité d'enseignement, chargé de la mise en œuvre de l'évaluation HCERES, montage de projets, direction de composante, direction d'unité, etc.). Or, ces tâches chronophages ont une valeur ajoutée jugée souvent faible par celles et ceux qui les accomplissent (faible rémunération, reconnaissance modérée dans la carrière). Certes, celles-ci s'accroissent dans toutes les disciplines, mais toutes n'ont pas connu une telle augmentation des effectifs étudiants, comme on l'a indiqué précédemment. En outre, le déséquilibre lié à la répartition des ECG fragilise l'utilité sociale et sociétale du métier (taux d'encadrement insuffisant, sentiment d'impuissance face aux demandes croissantes de soutien des étudiants). Enfin, l'activité de chercheur peut être déstabilisante car tous les projets n'aboutissent pas. Les recherches nécessitent du temps, leurs résultats sont parfois in fine décevants ou peu reconnus par les pairs (recherche peu innovante, problématique à consolider, méthodologies inadaptées, traitements insatisfaisants, apports managériaux limités, etc.), les projets n'obtiennent pas de financement, etc.

\section{Une évaluation en continue par ses pairs}

Les ECG ont le sentiment d'être évalués constamment sur les trois dimensions du métier. Leur carrière démarre par une évaluation et cela ne cesse jamais, individuellement ou collectivement. En voici quelques exemples. Les évaluations individuelles concernent la soutenance de la thèse de doctorat, la qualification CNU (tant qu'elle existe), le recrutement par un comité de sélection, les avancements, primes (PEDR, PEP), soumissions de communications ou d'articles. Concernant ce dernier point, soulignons que la soumission d'un article dans une revue classée (CNRS, HCERES, FNEGE) est un processus stressant et long. II dure plusieurs mois, parfois années, car le papier est repris jusqu'à quatre fois suite aux demandes de modifications des évaluateurs, qui peuvent aussi le rejeter à chaque étape. Les évaluations collectives examinent les formations et les laboratoires (HCERES), les projets de recherche soumis (AAP européens, nationaux, locaux, dans son université). Notons que la multiplication des AAP est une tendance actuelle forte du métier. Dit autrement, nous sommes sans cesse en quête d'argent pour exercer notre métier ! Cette impression d'évaluation permanente crée de la compétition, renforcée par le faible nombre de postes de professeur des universités, le nombre de promotions limité, etc. 


\section{Évolution du métier et rémunération}

Les ECG font face à des mutations dans l'exercice de leur métier, liées à des exigences nouvelles (Imelda et al., 2011) : concurrence accrue en recherche, demandes sociales, attentes des étudiants, processus de Bologne, croissance de la formation continue ou en apprentissage, notamment. Mais la rémunération ne suit pas, et la précarisation augmente. Le salaire n'est pas la motivation principale des ECG. Pour preuve, la comparaison avec les carrières du privé : à niveau de responsabilité et de diplôme équivalent, le fonctionnariat n'est pas avantageux. Prenons la rémunération mensuelle (2020) d'un maître de conférences en début de carrière. Elle est de 2169 euros brut et de 2436 euros brut au bout de deux ans ${ }^{5}$. Les charges pédagogiques et administratives ne sont pas ou peu rétribuées. Cela occasionne dans la sphère privée incompréhensions et malentendus, voire des situations cocasses comme celles d'un fils qui demande naïvement à sa mère combien de voyages aux États-Unis elle pourra lui offrir grâce à sa nouvelle prise de fonction au sein de l'Université ! " Travailler plus pour gagner plus » ne s'applique pas vraiment aux enseignants-chercheurs exerçant dans les universités françaises. En effet, aux États-Unis, au Canada, en Suisse, en Grande-Bretagne, les rémunérations sont nettement plus attractives pour un nombre d'heures d'enseignement bien inférieures.

\section{Do you speak english?}

La pratique de l'anglais devient une nécessité pour l'enseignement et la recherche. Si cela est cohérent avec une volonté de rayonnement et de diffusion internationale, cela confine parfois à l'absurde. Déjà, l'usage de l'anglais n'est pas toujours aisé. Le niveau des ECG est très disparate et lié à aux formations et parcours antérieurs de chacun. Cela peut devenir une source de discrimination, voire de mépris de la part de certains collègues. Des possibilités de formation existent, mais encore faut-il avoir le temps de les suivre. Parmi les outils d'apprentissage, les ECG adoptent ceux relevant de l'intelligence artificielle (DeepL, Linguee), ou encore des vidéos en ligne sur diverses plateformes publiques (ENT universitaires) ou privées (YouTube). L'anglais devient donc une langue de travail pour les ECG francophones, parfois avec peu de discernement. En voici quelques illustrations.

D'abord, quand un enseignant-chercheur francophone fait cours à des étudiants également francophones en anglais avec un niveau de maîtrise approximatif des deux côtés, cela aboutit à un appauvrissement du contenu enseigné. En effet, sauf en cas de fluence parfaite et partagée, notre capacité à nous exprimer, à nuancer le propos est toujours supérieur - et parfois largement ! - dans notre langue maternelle. Ensuite, lors d'ateliers doctoraux dont la langue imposée est l'anglais (par automatisme), alors que les doctorants présents, issus des rives de la Méditerranée (Italie, Espagne, Maghreb, France, Liban) sont tous francophones, le plus souvent, personne parmi les organisateurs n'ose poser la question de la préférence linguistique. Enfin, lors d'une évaluation d'ECG, un travail diffusé en anglais a tendance à être mieux apprécié qu'un rendu équivalent en français, comme si une recherche

5 Pour un professeur des universités, 3102 euros brut au début de la carrière, et 3458 euros brut au bout de deux ans. 
en anglais était intrinsèquement supérieure... En conséquence, certains collègues choisissent de produire des recherches exclusivement en langue anglaise. Qu'en est-il alors de notre mission de diffusion de la connaissance auprès des acteurs non académiques ou la francophonie?

\section{Améliorer sans détruire}

On ne peut comparer la place d'un professeur des universités dans la société française aujourd'hui avec ce qu'elle était il y a un demi-siècle, ou davantage (Beaud et Vatin, 2020). Le métier doit certes évoluer mais en préservant les valeurs qui en font la richesse et l'utilité. Or, celles-ci sont menacées par un processus de réformes en cours.

\section{Le statut national en question}

Le métier d'universitaire a connu depuis 1984 de profonds bouleversements. L'apparition d'une université de masse a accru les charges de travail et le choix d'une gestion à moindre coût a reporté, en plusieurs vagues, de nombreuses fonctions sur les épaules des enseignants-chercheurs (Annoot, 2011). Plus récemment, deux nouvelles lois affectent l'université : la loi n²018-166 du 8 mars 2018 relative à l'orientation et à la réussite des étudiants (dite loi ORE), et la loi n 2020-1674 du 24 décembre 2020 de programmation de la recherche pour les années 2021-2030 et portant diverses dispositions relatives à la recherche et à l'enseignement supérieur (dite loi LPR). Les deux sont simplement mentionnées pour illustrer à nouveau le schisme entre enseignement et recherche, selon le point de vue de l'État central. Attardons-nous sur la seconde, qui remet en question le statut des enseignants-chercheurs, notamment son caractère national.

La LPR constitue une rupture brutale et sans sommation puisque les points touchant à ce statut ont été introduits par amendement au cours du débat parlementaire en nocturne au Sénat, à savoir la création des emplois de "professeurs juniors" et la suppression de la qualification 46.1 par le CNU. Le statut de " professeurs juniors » offre une procédure accélérée pour l'accès au professorat de chercheurs - étrangers ou français - considérés particulièrement prometteurs. Après une période comprise entre trois et six ans en tant qu'agents contractuels de droit public, ces professeurs juniors accèderont directement au corps des professeurs des universités. Au-delà de l'étonnement suscité par ce bond de comète, interrogeons-nous sur les critères de sélection et le profil des sélectionneurs. À partir de quels critères est-on considéré comme " prometteur » en tant que chercheur, et qui en décide?

Chaque université pourra définir ses critères, composer ses comités de sélection et pourvoir jusqu'à la moitié de ses recrutements annuels (avis du Conseil d'État du 9 juillet 2020) (Beaud et Vatin, 2021). Au vu du caractère " prometteur » de ces futurs collègues, on pourrait supposer que la liste de leurs compétences et qualifications soit extrêmement longue. Qu'en est-il ? Pour candidater, il faudra être titulaire " d'un doctorat ou d'un diplôme équivalent ". Nouvelle surprise! Les maîtres de conférences promus à des postes de professeur des universités doivent être docteur, réussir l'agrégation du supérieur (pour les disciplines agrégatives dont font partie les 
sciences de gestion et du management), ou obtenir une HDR (le plus haut diplôme universitaire), incluant des activités de recherche significatives, et être qualifié par le CNU (jusqu'en 2020). Ainsi, les professeurs juniors recrutés sur un seul critère, que satisfont tous les maîtres de conférences, sont considérés comme plus prometteurs! Notons au passage que l'enseignement a disparu des exigences de recrutement, qui restent imposées au « tout venant » des enseignants-chercheurs.

Quant à la suppression de la qualification par le CNU pour l'accès aux fonctions de professeur des universités et son maintien pour les maîtres de conférences pendant une durée expérimentale de quatre ans (en sciences de gestion et du management), elle est la seconde atteinte majeure au statut national des enseignants-chercheurs (Paché, 2021b). L'un des arguments repris par la ministre de l'Enseignement supérieur concerne la possibilité pour « des milliers de maître de conférences [d'accéder] à des promotions dans le corps des professeurs des universités ». Force est pourtant de constater que de nombreux maîtres de conférences, titulaires d'une HDR et qualifiés par le CNU, n'ont pas obtenu le statut de professeur des universités, faute de postes à pourvoir, sachant que c'est le ministère de l'Enseignement supérieur, de la Recherche et de l'Innovation qui décide annuellement de leur nombre. Au final, la LPR a été fortement décriée, sur le fond comme la forme, par les universitaires français. Serait-ce seulement un malentendu?

\section{Le discours de la réforme}

La LPR interroge à la fois comment devenir enseignant-chercheur (entrer dans la carrière) et comment être enseignant-chercheur (faire carrière). Or, l'attractivité de notre métier nécessite de donner des lignes claires et des perspectives aux doctorants qui s'engagent pour plusieurs années dans un processus de recherche et de formation. Que vend-on aux aspirants enseignants-chercheurs? Notre éthique professionnelle et personnelle interdit de leur vendre du rêve! Comment inviter des étudiants prometteurs sur les chemins de l'enseignement et de la recherche si, nous, universitaires en poste, ignorons de quoi sera fait le métier l'année prochaine, pour tous, mais en particulier, pour les nouveaux entrants ? La disparation annoncée, avec brutalité, sans concertation, de la qualification pour l'accès aux fonctions de maître de conférences balaie d'un revers de la main les repères sur le chemin du métier d'enseignant-chercheur, sans en proposer de nouveaux. La prise de décisions politiques intensément engageantes a précédé la concertation. Du point de vue du management public, c'est plutôt cocasse.

Au-delà des modalités de réforme (son processus de construction), attardons-nous sur une de ses conséquences : la fragilisation du système universitaire de recrutement. Les enseignants-chercheurs ont besoin d'un cadre et d'un statut national s'appliquant à toutes les disciplines scientifiques, et dans lequel chacune (sections $\mathrm{CNU}$ ) préciserait si nécessaire les modalités de recrutement, de progression et de fonctionnement dans le respect du cadre national. Est-il vraisemblable d'imaginer qu'une diminution de la sélectivité des universitaires aboutisse à terme à améliorer l'attractivité et la qualité des profils d'enseignants-chercheurs ? Augmenter l'attractivité par une diminution des exigences d'entrée est une vraie mauvaise idée. Souhaite-t-on recruter et travailler avec des universitaires au rabais pour nos universités ? Qu'avons-nous à y gagner collectivement, socialement, sociétalement ? 
Imaginons que le métier d'enseignant-chercheur soit un château médiéval. Désiret-on une réforme qui le transforme en merveilleux château Renaissance ou, au contraire, qui le démantèle pierre par pierre jusqu'à obtenir une ruine posée sur un terrain vague ? De quoi s'enorgueillit-on : d'avoir conservé Chambord ou détruit les Tuileries ? Lequel des deux entraine le plus de retombées positives aujourd'hui ?

Les enseignants-chercheurs, comme d'autres corps ou professions, ne sont pas, par principe ou nature, réfractaires au changement. Mais comme d'autres, ils n'ont aucune raison d'accepter n'importe quel changement. Les discours des politiques face aux réticences qu'engendrent certaines réformes - bizarrement, celles qui tendent à réduire les acquis sociaux et avantages professionnels - insistent sur la nécessité d'accentuer la pédagogie. Les oppositions seraient dues aux malentendus. Elles s'expliqueraient par l'absence de compréhension, fine ou globale, des changements proposés. Un surcroît de pédagogie et, miracle, toute résistance disparaîtra. Vive la pédagogie! Vive la réforme! Dans les faits, l'opposition peut avoir pour origine et principal moteur, bien au contraire, la claire compréhension du contenu et des conséquences de la réforme en question. Elle naît de la claire appréciation des changements proposés par ceux qui en sont destinataires, en capacité de calculer un ratio avantage / inconvénient, et de débusquer un piétinement des valeurs d'égalité, d'équité, de justice, de service public. Voilà autant d'éléments que sont parfaitement capables de mesurer les destinataires de premier, de second plan, voire plus, de ces réformes.

\section{Haro sur les libertés?}

La LPR est le dernier avatar de textes contribuant à saper les libertés des universitaires, dont les vertus ont été soulignées. Le malaise des enseignants-chercheurs est réel face à cette tendance. Comment se manifeste-t-elle ? Par une généralisation et une intensification des contrôles dans toutes les facettes de notre métier. Les reportings (formulaires des comités de sélection, feuilles de temps des projets européens, etc.) se multiplient, sous couvert de conformité, de qualité, de course à l'excellence et au label, voire de transparence. Au-delà de cet affichage, nos organisations sont très préoccupées par de potentiels recours. La parade? La standardisation à outrance. Outre la fragilisation de la liberté universitaire, elle se révèle contre-productive : malgré la quantité de rapports produits, les décisions liées aux enseignements, à la recherche et à la vie collective restent opaques. Celles-ci ne sont pas expliquées sincèrement par peur des recours. La transparence est sur toutes les lèvres, mais elle tend dans les faits à s'estomper. Face à la candidature d'un collègue ou d'un étudiant, en utilisant des "éléments de langage " pour justifier une acceptation ou un refus, les concernés ne peuvent s'emparer d'une critique détaillée et constructive. Le rétrécissement des libertés et l'affichage trompeur de transparence créent des conditions défavorables à la créativité, fondamentale dans notre métier. La perte de repères engendrée par la LPR aggrave cette situation. Elle fragilise les fondements des décisions et provoque d'autant les recours. 


\section{Conclusion}

Le constat face aux inégalités sociales est sans appel : elles se creusent. L'université française accueillait plus d'un million et demi d'étudiants en 2020. Quel formidable levier pour agir. Inclusion, diversité des profils des étudiants et des enseignants-chercheurs, insertion professionnelle, approche par les compétences, les besoins de politiques publiques favorables à l'enseignement supérieur et à la recherche sont considérables. La communauté universitaire, et chaque section $\mathrm{CNU}$, sont prêtes à s'investir dans la concertation. Réformer, c'est innover en intégrant la richesse de l'existant, donner des moyens pour engager les transformations, améliorer sans détruire. L'attractivité du métier est mise en péril par la dureté des discours et les réformes non concertées entraînent frustrations, incompréhensions et démotivations. Ce métier est formidable, passionnant, stimulant et gratifiant à plus d'un titre, mais aussi frustrant et éreintant parfois. Les ECG attendent de véritables évolutions positives de leur métier et sont prêts à y prendre une part active. 



\title{
La recherche à forts impacts sociaux
}

\author{
Enjeux scientifiques et utilité sociétale
}

\author{
Marc Bonnet, Florence Noguera, Jocelyn Husser et Françoise Goter
}

Ces dernières années, la charge de travail et les exigences pour les enseignants-chercheurs en sciences de gestion et du management se sont multipliées. L'intensification des critères qualité appliqués à la gestion des diplômes et aux laboratoires de recherche en est un exemple. Le métier d'enseignant-chercheur est devenu protéiforme. À côté de ses missions d'enseignement et de gestion des diplômes, il doit développer des activités de recherche nombreuses : réalisation de travaux de recherche fondamentale ou appliquée, implication dans les instances des universités et nationales (CA, CS, CEVU, CNU, etc.), coordination d'équipe pédagogique, direction et animation d'équipe de recherche, encadrement doctoral et d'HDR, recherche de contrat public / privé, publication des travaux dans des revues scientifiques classées et participation à des colloques / congrès nationaux et internationaux. Avec la crise liée à la pandémie, les enseignants-chercheurs ont dû accélérer leur apprentissage et leur maîtrise d'outils numériques pour enseigner et diffuser des connaissances via des supports innovants.

Un malaise est observé dans la profession en raison de la dégradation des conditions de travail : mécontentements dus à la bureaucratisation croissante du métier, à la dévalorisation de la pédagogie, à la difficulté à rechercher des financements de travaux de recherche, à la difficulté de concilier vie privée et vie professionnelle qui s'est accélérée depuis la crise liée à l'épidémie. Au demeurant, les multiples missions renvoient à des critères d'évaluation des enseignants-chercheurs qui ne sont pas toujours clairement affichés ou bien pris en compte selon les sections du CNU et les comités de sélection. Dans l'examen des dossiers en matière de qualification, de promotion et de PEDR, on observe également une grande diversité des critères d'évaluation et des pratiques entre les sections.

Simultanément, la demande sociale d'engagement des différentes parties prenantes (universités, écoles, financeurs de la recherche publics et privés), dans des recherches ayant des impacts sociétaux, est de plus en plus forte, et il s'agit d'un pan peu valorisé dans les missions et travaux de recherche et critères d'évaluation. Dans 
la pratique, il apparaît que les critères actuels d'évaluation scientifique des enseignants-chercheurs n'intègrent pas suffisamment les impacts sociétaux des travaux de recherche et les rapprochements des travaux de recherche avec une demande sociale. Si les missions de la recherche ont progressivement évolué d'une science ayant comme seul objet la recherche de la connaissance à une science " utile ", devant démontrer son impact sociétal, pour autant, dans l'évaluation des enseignants-chercheurs, l'impact sociétal n'est pas suffisamment intégré, sans doute en raison du très large spectre que peut englober le terme " impact sociétal ».

L'objectif du chapitre est de se demander comment répondre aux exigences des parties prenantes de la recherche et comment mieux valoriser les recherches socialement responsables afin de renforcer leur visibilité au niveau scientifique et au niveau professionnel. Plus précisément, il convient de se demander comment accroître la visibilité des apports de la recherche en gestion des enseignants-chercheurs en France et valoriser les recherches à forts impacts sociétaux dans l'évaluation des enseignants-chercheurs et des unités de recherches. La contribution met en évidence les attentes des parties prenantes vis-à-vis de la recherche, les méthodes de prise de distance, d'analyse et de validation des résultats scientifiques. L'enjeu est de montrer en quoi les recherches-actions, à visée transformative, créent de la connaissance actionnable pour le monde socio-économique.

\section{Montée de l'exigence des parties prenantes de la recherche}

La demande sociale ne s'exprime plus uniquement à travers le classement des écoles et des universités ou encore la publication de nouvelles connaissances scientifiques. Les parties prenantes qui financent les recherches attendent une contribution explicite aux enjeux de la société. Les entreprises et organisations demandent aux chercheurs de répondre à des difficultés croissantes dans un contexte de complexité. Le secteur public recherche des modes de gestion innovants (cas de l'hôpital), qui pourraient lui permettre de rendre plus pertinents ses efforts de transformation. Quant aux autres disciplines des sciences sociales, telles que l'économie, la sociologie ou la psychologie, elles ont tout intérêt à coopérer avec les sciences de gestion et du management pour construire des modes d'action répondant aux constats de blocages de ces disciplines.

Les parties prenantes qui financent la recherche sont multiples et ont des enjeux et des objectifs spécifiques. Par exemple, dans le cadre de cofinancement des allocations doctorales, les directions de la recherche, du transfert technologique et de l'enseignement supérieur des régions, à côté des critères thématiques et domaines d'innovation du territoire (écosystème), introduisent, y compris dans des disciplines qui y échappaient jusque-là, les sciences humaines et sociales, des critères pour l'évaluation des projets, tel que l'échelle TRL (technology readiness level). Cette échelle a été développée dans le domaine des sciences de l'ingénieur et reste fortement marquée par ses origines (NASA et secteur de la défense, puis largement reprise par l'industrie aéronautique).

L'échelle a été adaptée aux sciences humaines et sociales et aux sciences de gestion et du management dans l'objectif d'apprécier le potentiel de valorisation des résultats des projets de recherche. Les neuf niveaux de l'échelle permettent 
d'apprécier le processus de production de connaissances " actionnables » sur le terrain (Noguera, 2018), le niveau 1 étant le plus faible et le niveau 9 le plus élevé. Cette échelle permet de vérifier que le produit de la recherche est mobilisé et utilisé par les publics auxquels il se réfère (Verstraete et Philippart, 2019). Les entreprises et les organisations publiques ou privées sont également des parties prenantes de la recherche au travers de convention de recherche partenariale publique-privé contractualisée avec une unité de recherche ou de contrats de collaboration de recherche type Convention Industrielle de Formation par la Recherche (CIFRE), encore peu développés en sciences de gestion et du management.

Cette demande sociale des acteurs du monde socio-économique pour les chercheurs en GRH porte, par exemple, sur la recherche de performances notamment à travers la mise en place de modes d'organisation du travail innovants, l'amélioration de la santé et le bien-être au travail, l'accompagnement de certaines grandes enseignes qui aspirent à des pratiques managériales socialement responsables et durables, l'aide aux entreprises en difficultés économiques, l'accompagnement de la transformation digitale de la fonction RH elle-même (comment utiliser le potentiel proposé par le numérique pour améliorer les processus $\mathrm{RH}$ ?), et de la transformation numérique globale (évolutions des emplois et des compétences, organisation du temps de travail, régulation des usages numériques et protection des données).

Dans le secteur public, la demande sociale porte sur la recherche de méthodes et d'outils d'évaluation des impacts socio-économiques des dispositifs publics (Savall et Goter, 2021). Les collectivités territoriales souhaitent par exemple être accompagnées pour améliorer leurs pratiques de redevabilité (rendre des comptes aux usagers) et développer plus de démocratie participative. Les baromètres du bien-être des usagers et les cartographies dynamiques sont utiles mais ne suffisent pas pour orienter efficacement les politiques publiques. Dans le secteur de la santé, la demande sociale est également très forte. Le mouvement de normalisation des activités de santé s'est accompagné d'une transformation des technologies et de l'organisation du travail qui impacte de nombreux métiers de santé. Des conventions de recherche entre les laboratoires de recherche, par exemple l'Association Nationale pour la Formation du Personnel Hospitalier (ANFH) ou des groupements hospitaliers de territoire, ont été contractualisées avec des chercheurs pour que ceux-ci accompagnent les praticiens dans ces transformations.

Enfin, l'une des parties prenantes majeures de la recherche est bien évidemment constituée par l'ensemble des doctorants. L'accompagnement tant académique que professionnel constitue le cœur de la mission des enseignants-chercheurs d'aujourd'hui. Il s'agit à la fois d'assurer le développement intellectuel des doctorants, mais aussi de leur assurer l'impulsion académique et professionnelle et continuer ainsi, au niveau de notre métier, à promouvoir l'ascenseur social. Les enseignants-chercheurs travaillent ainsi de plus en plus sur l'accompagnement individualisé des doctorants jusqu'à l'insertion professionnelle, soit dans le monde académique, soit dans le monde de l'entreprise. Cette mission que constitue l'accompagnement des doctorants et jeunes docteurs se poursuit jusqu'au suivi de leur devenir professionnel. L'obtention d'un premier contrat dans le monde du travail, au niveau attendu pour un docteur, constitue un critère d'excellence universitaire dans le domaine des sciences de gestion et du management. 


\section{Enjeux liés à la visibilité des apports de la recherche en gestion en France}

La recherche française a déjà produit depuis plusieurs décennies des recherches sociétalement utiles, mais cela est peu visible et peu valorisé. Mieux valoriser les recherches socialement responsables et les reconnaître dans les carrières sont donc des enjeux légitimes, car elles différencient fortement les sciences de gestion des autres disciplines de sciences sociales. Certaines méthodes de recherches de type cas de recherche, observations participantes, recherches actions et recherches-interventions, identifient les apports de la recherche en termes d'impacts sur les pratiques managériales. La recherche-intervention est une méthodologie de recherche à fort impact managérial et sociétal (Savall et al., 2019). Elle se caractérise par une immersion dans l'entreprise et l'organisation en vue de la conduite d'une intervention, c'est-à-dire accompagner ou susciter une transformation des organisations. II peut s'agir également d'expérimenter de nouveaux modèles de management, des outils de gestion ou même de chercher à les conceptualiser (Noguera, 2018 ; Plane, 2018). Le chercheur est présent et acteur de l'organisation, il interagit, et cette intervention a fait l'objet d'une négociation entre le chercheur et l'équipe dirigeante. Cette méthode est privilégiée pour approcher le terrain et les situations de management.

La méthode de recherche pour l'action consiste à produire des connaissances et des concepts qui permettent de penser des trajectoires dans lesquelles les entreprises pourraient s'engager (Hatchuel, 1994). L'intérêt d'une telle démarche se situe dans la production de connaissances théoriques à partir d'observation et d'investigation participante du chercheur sur le terrain. Une recherche-intervention comporte plusieurs caractéristiques méthodologiques car la mise en tension de postures différenciées chercheur et praticien nécessite confrontation, analyse et distanciation pour éviter de privilégier la visée scientifique par rapport à la visée pratique, ou vice versa (Noguera et Plane, 2020). Cet équilibre est plutôt à voir comme une conciliation des objectifs qui peuvent varier en fonction du temps, mais concrètement la recherche-intervention propose systématiquement deux " livrables » : I'un managérial, l'autre en recherche.

La co-production de connaissances positionne les recherches-interventions dans le paradigme constructiviste puisque le changement et le processus de transformation de l'objet étudié ne sont pas des problèmes pour le chercheur mais des opportunités de recherche. Par exemple, le fait de travailler sur toute une profession (notaire, professions libérales, etc.) permet de générer des cadres de réflexion et des préconisations managériales intégrant une exhaustivité des contextes professionnels rencontrés. Le temps de l'enseignant-chercheur est un actif spécifique pertinent s'il est mobilisé dans une optique de distanciation / conceptualisation, puis de restitution / opérationnalisation managériales au service d'un métier donné. Les liens entre le monde académique et celui des managers en gestion doivent permettre des aller-retours féconds entre universités et organisations : les apports du scientifique en immersion dans les entreprises permettent d'apporter des outils originaux et opérationnels aux professionnels.

La valorisation de la pensée académique par la création d'outils de gestion, novateurs, efficients et adaptés au monde des affaires apporte une visibilité aux sciences de gestion. Cela implique un rapprochement des entreprises et des 
institutions universitaires où le respect du temps et de l'investigation scientifique de création multiple débouche sur des outils de formation et d'application robustes. II en va ainsi de l'éthique des affaires et de la Loi Sapin II en particulier. Les cadres conceptuels cognitifs et comportementaux élaborés par le monde académique en situation d'immersion professionnelle peuvent déboucher sur une liste exhaustive de situations managériales, dilemme permettant des guides de conduites à tenir et de formations robustes pour la mise en œuvre quotidienne de l'éthique des affaires. L'élaboration de normes professionnelles sociétales, sociales, environnementales et de gouvernance, ainsi que la co-construction de normes avec le monde professionnel, permet de créer des outils de mesure originaux pour comptabiliser et valoriser les efforts à long terme des organisations en termes sociétaux.

\section{Mieux valoriser les recherches à forts impacts sociétaux}

L'accroissement de la demande sociale des parties prenantes de la recherche et le développement de recherche de terrain conduisent à réfléchir à de nouvelles pratiques d'évaluation intégrant des critères à fort impact auprès du monde socio-économique. Plusieurs voies de progrès sont possibles. Tout d'abord, la création d'une rubrique spécifique dans les critères d'évaluation des dossiers des enseignants-chercheurs permettrait d'identifier plus précisément les apports de la profession. II suffirait d'accroître le spectre de l'évaluation avec d'autres indicateurs tels que les contrats, matériaux et terrains de recherche. Nous pourrions également proposer une cartographie des recherches ayant des impacts sociétaux sur un site Internet, qui pourrait être hébergé par la FNEGE. Dans une autre dimension, les missions réalisées par les vacataires-praticiens pourraient être encouragées. Elles ont le double avantage de soulager la charge de travail des enseignants-chercheurs et d'enrichir la qualité des connaissances des entreprises et des organisations. Celles-ci sont importantes pour la mise à jour des contenus des programmes, pour réaliser des recherches pertinentes. Un tel dispositif aiderait aussi les praticiens à formaliser et à conceptualiser leurs savoir-faire tacites. C'est ainsi reconnaître les activités de scholar practitioners.

Ensuite, les sciences de gestion et du management représentent un champ disciplinaire propice à la création de brevets. Penser en termes de brevets place les enseignants-chercheurs dans une double perspective intéressant les organisations : (1) en termes de distanciation-conceptualisation; et (2) en termes de transmission-opérationnalisation du savoir. Travailler dans une perspective de brevets crédibilise les préconisations managériales formulées par les enseignants-chercheurs et répond à la question de la valorisation de la recherche académique. L'enregistrement de brevets interroge à son tour les institutions : élargir le champ des critères de dépôt de brevets pour les sciences de gestion est un défi sociétal pertinent alors même que les organisations recherchent constamment des outils opérationnels pertinents pour le pilotage de leurs entités.

Les associations savantes peuvent aussi jouer un rôle dans la valorisation des recherches à forts impacts sociaux. Par exemple l'AGRH, distingue chaque année deux thèses en GRH. Dans le cadre de ces deux prix, sont évalués les qualités méthodologiques de la recherche, le caractère innovant du sujet dans le domaine de la GRH et les qualités formelles et pédagogiques du manuscrit. Ce qui les distingue d'une 
trentaine de thèses évaluées par an est l'importance respective donnée à la dimension académique et aux implications managériales. II est apprécié par les évaluateurs que la thèse dédie le dernier chapitre à la présentation d'apports plus riches et pertinents en termes d'implications managériales. L'objectif est d'encourager les recherches qui constituent un véritable apport pour l'entreprise. Une autre voie, assez simple, est d'inciter les enseignants-chercheurs à valoriser les travaux scientifiques dans des revues professionnelles, à fort impact socio-économique, sociétal (exemples en GRH : la revue Personnel, la revue Santé $R H$, la Revue des Cadres).

Ces dernières années, de nouveaux canaux de diffusion se sont développés et mériteraient d'être davantage mobilisés par la communauté académique. C'est le cas des chaînes télévisées ainsi que des observatoires tels que FNEGE Médias, Xerfi Canal, The Conversation, l'observatoire Action sociétale et action publique (ASAP), etc., offrant une tribune aux enseignants-chercheurs pour présenter leurs résultats de recherche et pour diffuser des savoirs autour de l'actualité générale et de la culture scientifique. De tels supports s'adressent aux citoyens, étudiants, chercheurs, professionnels et institutionnels. L'objectif n'est pas simplement de nourrir les débats scientifiques et publics mais d'avoir un souci de transparence et d'utilité sociétale. À titre d'exemple, l'observatoire ASAP a été créé dans le cadre de la Chaire ENA-EN$\mathrm{SCl}$-Polytechnique-Sciences Po sur l'innovation publique. Ces nouveaux outils ont le double avantage d'accélérer la diffusion de résultats de recherche, qui prend souvent plusieurs mois voire plusieurs années via les supports traditionnels.

\section{Conclusion}

La compatibilité des exigences des différentes parties prenantes de la recherche peut être obtenue par le développement et la reconnaissance des travaux scientifiques ayant des impacts sociétaux. En effet, la valorisation de recherches socialement responsables dans les multiples dimensions du métier d'enseignant-chercheur est réaliste. La démarche renforcera la légitimité de notre discipline au niveau des ministères et de la société en établissant des liens systématiques entre pratiques, recherche et enseignements. Les résultats issus de la recherche académique se traduisent en termes de préconisations ou d'innovations managériales. La variété et les enjeux qui transparaissent dans les demandes sociales soulignent bien la nature ainsi que le niveau de responsabilité des enseignants-chercheurs aujourd'hui. La bonne nouvelle est que même si les résultats des travaux scientifiques ne sont pas encore suffisamment visibles, de nombreux outils et méthodes permettant de mieux valoriser les impacts socio-économiques de ces travaux se développent. Ils témoignent ainsi de la fécondité et de la pertinence du métier d'enseignant-chercheur en sciences de gestion et du management qu'il convient de mieux identifier et valoriser par des actions de communications appropriées. 


\section{La valeur de l'indépendance}

Jean-Philippe DeNIS

"Une ville se fane

Dans les brouillards salés

La colère océane est trop près

Les tourments la condamnent

Aux écrans de fumée

Personne ne s'éloigne du quai »

Francis Cabrel, Hors-saison (1999)

Nous étions en 2010. Alors que je venais moi-même de créer un blog intitulé Journal d'un universitaire, Pierre Dubois, auteur du blog Histoires d'universités ${ }^{1} \mathrm{~m}^{\prime}$ interpellait sous la forme d'un commentaire de l'un de mes billets :

Les jeunes chercheurs (doctorants et néo-docteurs) en sciences humaines et sociales, économie et gestion ont-ils intérêt à publier immédiatement leurs résultats en ligne (pour se faire connaître, pour participer au débat public), ou ont-ils intérêt (pour la carrière) à attendre de publier dans une revue labellisée AERES, quitte à ce que leurs résultats soient datés, obsolètes ?

L'invitation à m'engager dans certains des débats sur le futur des universités était donc claire. En première approche, j'ai cependant jugé que, sur l'université française et ses évolutions, les points problématiques que je pouvais soulever avaient été déjà bien mieux exprimés ailleurs, par d'autres. La tentation était donc grande de renvoyer aux remarquables contributions du numéro spécial de la revue du Mauss qui est consacré à cette question (Caillé et Chanial, 2009), ou encore à la contribution de Vatin et al. (2010) publiée dans les pages de la Revue française de gestion.

1 [https://histoiresduniversites.wordpress.com], consulté le 13 juin 2021. 
Des textes qui sont assurément à lire avant de pouvoir engager des réflexions sérieuses. Des textes qui ouvrent surtout le débat davantage qu'ils ne le concluent. Des textes qui me paraissent importants, notamment lorsqu'ils établissent quelques vérités de bon sens et contribuent ainsi à la grandeur du projet que j'ai toujours vu personnellement dans le travail de l'intellect : moins apporter des réponses que contribuer à élever le débat, pour reprendre la jolie formule par laquelle Hirschman (2014) conclut son ouvrage passionnant : Les passions et les intérêts. J'ai finalement jugé que se contenter de renvoyer à ces textes était une facilité. Que je pouvais peut-être, à la lumière de ma discipline - le management stratégique - apporter un regard complémentaire. Puisque dans ma discipline, on considère généralement que le vécu a du sens, que le diable, souvent, se cache dans les détails, alors j'ai fini par me décider à apporter une confession et un témoignage dont je pourrais essayer de tirer quelques leçons. Ces propos assument une entière subjectivité et n'engagent que leur auteur pour formuler les précautions d'usage habituelles ${ }^{2}$.

\section{Retour d'expériences}

La confession, d'abord. J'ai eu la chance d'avoir des vies antérieures à l'université, notamment d'avoir été confronté à la vie d'un grand cabinet de conseil en stratégie alors que j'étais engagé dans un processus de thèse de doctorat; et puis, une fois entré à l'université, d'avoir poursuivi, de temps à autre, à côté, puisque l'honneur m'était fait de me demander d'apporter le concours de mon expertise. La question qui s'est alors souvent posée à moi a été la suivante : faut-il, d'abord, entrer à l'université, avec une rémunération plusieurs fois inférieure à celle qui m'était proposée ailleurs (et ce alors même que j'étais à la fin de ma thèse encore à bonne distance de mes trente ans... mais déjà papa) ? Puis, plus tard, fallait-il quitter l'université au vu de la paupérisation qui y règne bien souvent, des langueurs administratives et des possibilités qui se laissaient entrevoir ailleurs ? À ces questions, j'ai toujours répondu en faveur de l'université et, plus généralement, surtout, du monde académique. Parce que dans aucun autre domaine ne se situe une telle concentration de personnalités aussi compétentes et diverses. Parce que nulle part ailleurs, je n'ai autant appris et continue d'apprendre autant. Parce que nulle part ailleurs, les talents ne sont si puissants et si nombreux que dans nos belles communautés académiques.

Alors, je déduis de cette confession que si de jeunes chercheurs ne songent qu'à faire carrière sans autre forme d'interrogations, alors je leur conseillerais sans doute que ce n'est pas vers l'université, ni même peut-être vers le monde académique, qu'ils devraient tourner leur regard. Je me permets de renvoyer donc la question : un chercheur qui n'aurait pas compris où il se situe entre le capital et le travail, et ce même s'il est plutôt privilégié en ce domaine (sa situation est finalement assez enviable), un chercheur qui n'aurait pas saisi quels intérêts il servirait d'abord par une obsession de publications " étoilées » et un désir exclusif de carrière, ce chercheur-là, ne ferait-il pas peut-être mieux d'arrêter la recherche sans attendre ? Sa naïveté ne risquerait-elle pas, à terme, de le rendre dangereux?

2 Le contenu du présent chapitre s'inspire largement de développements contenus dans Denis (2010, 2014). 
Avant d'apporter mon témoignage, pas tout à fait sans lien d'ailleurs avec cette confession, quelques précisions à destination de celles et ceux qui ne seraient pas au fait de la vie universitaire. On a beaucoup discuté du fait que le statut d'universitaire regroupe trois ensembles de tâches : l'enseignement, bien sûr ; la recherche ensuite, souvent - et à tort, j'y reviendrai - assimilée aux publications ; et les tâches administratives. Sur ce dernier point, des précisions s'imposent d'emblée. Elles sont, en effet, de deux ordres : celles qui relèvent du contact avec les étudiants (direction de diplômes, animation d'équipes pédagogiques, organisation de l'année, présidence des jurys, etc.), et celles qui relèvent davantage du politique (direction de département, de laboratoires, charges de mission diverses, vice-présidence, etc.). Cette seconde catégorie se subdivise elle-même en plusieurs sous-catégories : le politique prioritairement tourné vers I'interne, vers I'institution (conseil d'UFR, conseil scientifique ou de la vie universitaire, etc.) ; et le politique tourné vers l'externe, vers la participation à la vie de sa propre communauté scientifique, qu'il s'agisse d'être membre de jury de thèse ou d'HDR, d'évaluer des papiers et des articles, de participer à des congrès, y compris à l'autre bout du monde. Le tout sans oublier naturellement que ces deux sous-catégories peuvent se confondre dès lors qu'il s'agit d'organiser un colloque, par exemple, ce qui mêle des problématiques du rayonnement interne mais aussi externe.

Ces précisions posées, je peux entrer dans le vif de mon témoignage. J'ai connu de très près, pour y avoir été en poste, plusieurs universités. J'en ai fréquenté - et continue de le faire - bon nombre d'autres, en région parisienne ou en province. Je note que, partout, les responsabilités administratives de mes collègues - mais aussi les miennes - montent mécaniquement en puissance au fil de la carrière, en raison de l'attrait des formations de gestion pour les étudiants comme des compétences gestionnaires pour des tâches à dominante de politique interne. Ceci dans un contexte général de manque de "support logistique ", comme dirait Henry Mintzberg. Le tout sans oublier ces cours qu'il faut bien réaliser et renouveler puisque c'est dans l'essence même du métier et que l'on aurait choisi une autre voie si l'on n'avait pris quelque plaisir à voir progresser des étudiants, à contribuer à la formation de futurs collègues, à partager et à rendre un peu de ce que l'on a eu la chance de recevoir.

De telles tâches sont évidemment accaparantes. Elles font partie du métier que j'ai choisi. J'avais pleinement conscience en le choisissant que j'allais devoir les exercer. Je ne m'en suis jamais plaint tant elles ont été aussi l'occasion d'exercer ma profession sous de multiples aspects, tous extrêmement enrichissants. Je note simplement que dans ce contexte, l'activité de recherche - mais aussi, de pensée - finit toujours par devenir le parent pauvre : les moments qui y sont consacrés sont de plus en plus difficiles à " caser ». Même lorsque l'on s'astreint à une certaine discipline, lorsque l'on tient à une saine hygiène, ces moments s'avèrent surtout de moins en moins productifs : il en va du chercheur en quête d'idées neuves comme du sportif de haut niveau, seul l'entraînement régulier - pour ne pas dire quotidien - paie.

On comprend aussi aisément que toutes ces tâches ne requièrent pas des talents identiques et j'accepte volontiers l'idée que d'autres que moi auraient pu être plus efficaces dans l'accomplissement de chacune. Vouloir les assumer toutes relève dans tous les cas du tour de force, proche de l'impossible dès lors qu'il s'agit de les assumer sérieusement. Pourtant, grâce à toutes ces tâches, dans leur diversité, j'ai appris des 
choses. Pourtant, aussi étonnant que cela puisse paraître, j'ai toujours eu le sentiment que loin de s'opposer, elles pouvaient se conjuguer les unes les autres et être sources d'un enrichissement mutuel. Voilà pour le témoignage. Je déduis d'une telle confession et de ce témoignage deux brèves remarques pour esquisser des éléments de réponse, quand même, à la question soulevée par Pierre Dubois. Cette question qu'il pose, et qui agite tous les repas de thèse, tous les couloirs des congrès, a trait à la meilleure stratégie de publication à adopter.

La première, c'est que m'interroge, on l'aura compris, la notion même de carrière dans nos domaines. La notion me convient mal. Une carrière, après tout, n'est-ce pas d'abord l'ensemble des choix stratégiques personnels qui auront été faits, et ce en fonction du sens que chacun met dans son action ? Alors peut-être les lignes qui précèdent, et celles qui suivront, trahiront-elles quelques-uns de mes sentiments sur la question et qui n'engagent que moi... L'essentiel est à mon avis ailleurs et c'est ceci qui reste assez impensé lorsque l'on ne parle plus, comme c'est trop souvent le cas actuellement, que stratégie de publication en vue d'une carrière.

La seconde, c'est qu'en adepte d'Edgar Morin, je m'interroge : pourquoi penser en exclusive (débat public vs. revues labellisées) ? Après tout, si, dans les Humanités, tous les résultats sont probablement biodégradables - et ceci est singulièrement vrai en gestion - sont-ils pour autant appelés à devenir si vite obsolètes ? J'ai la faiblesse d'estimer qu'aucun des articles, chapitres d'ouvrage, et plus généralement des écrits que j'ai essayés de commettre, n'aient été appelés à une obsolescence aussi rapide. Bien sûr, j'ai pu faire des erreurs, pêcher par omissions, être d'abord condamné dans mon entreprise par l'étendue de ma méconnaissance. Mais jamais, je crois, je n'aurais eu le courage d'entreprendre chacune de ces publications si je n'y avais vu un sens autre que celui du bénéfice qu'elle pourrait me rapporter en termes exclusivement de carrière. J'ai donc la prétention déraisonnable de croire que si les résultats devaient être si vite obsolètes, alors ce sont peut-être les questions de recherche qui devraient être (re)posées.

N'est-ce pas donc la responsabilité première du chercheur - jeune ou moins jeune - d'interroger sans relâche la pertinence même des objets qu'il prend pour investigation et des projets de connaissance qu'il développe ? Bref, avant de réfléchir à la meilleure manière de publier, ne faudrait-il pas plus souvent (re)poser la question du sens du projet qui nous anime? Ceci aiderait aussi, peut-être, les plus jeunes à mieux supporter les refus des revues labellisées : n'oublions pas qu'un taux de rejet élevé permet désormais d'améliorer un classement! Je termine donc ces éléments de réponse en disant que c'est aussi aux jeunes chercheurs de faire cet apprentissage-là et de comprendre de quel jeu, parfois, ils pourraient n'être que les pantins.

\section{Étonnements}

À la lumière des éléments précédents, je peux maintenant entrer dans le vif du sujet plus général de l'évolution des universités et des questions qui m’ont été posées... comme dans celles qui ne l'ont pas été d'ailleurs. Longtemps - trop longtemps peut-être, je ne sais pas - à l'université comme ailleurs, la logique de coopération qui prévalait était une logique d’autorité mêlée à une logique de don. L'autorité, c'était bien sûr celle que conférait d’abord le titre : assistant, maître de conférences, 
professeur des universités, chacun aux missions spécifiques, et on pourrait de même parler des grandes écoles puisque les distinctions entre professeur assistant, professeur associé et full professor recouvrent globalement celles en usage au sein de l'université.

Le don, c'était celui dont faisaient montre toutes ces catégories qui espéraient, chacune, un contre-don. Ainsi du contre-don de la richesse issue des échanges d'abord avec des maîtres, ensuite avec des collègues; de celui de l'infini bonheur d'avoir le sentiment de progresser, d'être meilleur aujourd'hui qu'hier grâce à ceux qui méconnaissent moins que soi-même; de cette joie sans cesse renouvelée de pouvoir participer à des discussions où l'on essaye de penser le monde, où l'on cherche à comprendre, où le réel est d'abord source s'insatisfactions viscérales, toujours à (re)discuter, jamais tenu pour acquis. Sur la longue durée, ce contre-don, c'était celui, bien évidemment, d'avoir la satisfaction de voir ses propres progrès attestés par ceux que l'on respecte le plus et qui décidaient un jour, alors que vous le sollicitiez, d'accepter de faire de vous un pair.

L'autorité ainsi conférée progressivement au fil de la carrière donnait un sentiment intense de responsabilités : il provenait du bonheur des conquêtes réussies, mais aussi des défaites face à cette adversaire si rude qu'est la connaissance cultivée. Cela ne garantissait jamais, mais favorisait grandement, une éthique et une capacité d'expertise, dont l'essentiel était que l'une comme l'autre soient d'abord matinées d'une certaine culture : celui ou celle qui avait tant reçu savait combien il importait pour lui de rendre en donnant à son tour ; parce que les épreuves vécues rendaient conscient du fait que, dans les domaines de la connaissance et de la culture, rien n'est simple. Ainsi, le maître ne pouvait-il même parfois, ô bizarrerie de la connaissance, apprendre de l'élève?

Cette logique mêlant autorité et don a pu paraître insuffisamment productive, exiger trop de patience, être parfois source de trop d'injustices, tolérer trop d'écarts, récompenser insuffisamment les bonnes volontés, être trop rigide, autoriser trop de débordements (les cooptations tant décriées, les universitaires « à mi-temps ", etc.). Elle reposait surtout, je crois, sur un bon vouloir, sur une confiance et, j'ose ce mot qui a perdu tant de sens aujourd'hui, sur du respect : des autres, des diversités, de ceux dont on savait d'abord qu'on avait tant à apprendre. Quelque chose qui ressortit, en quelque sorte, d'une forme de cet esprit de famille aujourd'hui largement en crise... C'est tout ceci que les logiques organisationnelles portées par les transformations de l'enseignement supérieur (depuis la LRU jusqu'à la LPR) ont (délibérément) mis à mal. La double logique d'autorité et de don, on le sait - et l'université n'est pas ici un cas isolé - paraît finalement bien faible au regard de l'attraction " naturelle » que peut provoquer un échange marchand fondé sur le dédommagement individuel, au sentiment de liberté qu'il peut donner, au bonheur de l'acquisition instantanée qu'il peut (donner l'illusion de) procurer. En ce sens, la LPR comme la LRU, sous leurs masques d'évidence et de bon sens managérial, sont d'abord, me semble-t-il, un symptôme de quelque chose de plus profond.

Ainsi, progressivement d'abord, puis de manière incroyablement rapide et brutale depuis le milieu des années 1990, l'émancipation que promettait l'apprentissage à l'école, au collège, au lycée, dans le supérieur a cédé la place à un raisonnement volontiers utilitariste que l'on peut résumer en une espérance de cash flows futurs. Le taux de sélection - et donc de refus - à l'entrée, la qualité du réseau des anciens 
et des fréquentations, les salaires de sortie, le temps nécessaire avant que l'employabilité ne se transforme en emplois concrets sont devenus synonymes des plus hautes places sur les podiums des classements nationaux. La globalisation et la compétition internationale ont supposé de ne plus se satisfaire des classements d'envergure nationale. II fallait donc être visible partout. Le processus est engagé depuis longtemps et, contrairement à ce que l'on croit souvent, le classement de Shanghai a non pas provoqué mais plus simplement entériné ceci ; n'est-il pas d'ailleurs très instructif qu'il nous vienne de cette même Chine qui a constitué ses propres agences de notation financière?

Même les grandes écoles, qui formaient l'élite de la République, ont subi l'onde de choc d'une recherche qui serait égale à des étoiles et à des chiffres, puisqu'il fallait désormais des indicateurs simples pour permettre les benchmarks. La course s'est alors engagée, les étoiles se sont mises à fleurir partout. Ne reste plus donc qu'à attendre le critère, le vrai, l'ultime : celui qui rapportera la masse salariale (les capitaux investis) au classement pour juger de la pertinence des réorganisations voire, j'ose le dire, des éventuelles liquidations à envisager. Car en recherche, les taux de citation des revues dans lesquelles les chercheurs publient finiront, qu'on le veuille ou non, par s'imposer. Comme s'imposera le $h$ index individuel pour attribuer des primes tout aussi individuelles. Oui, c'est bien tout cela qui nous guette.

Alors, la LRU hier, la LPR aujourd'hui sont-elles par elles-mêmes responsables de ces évolutions? Les réécritures successives du statut des enseignants-chercheurs régi par le décret dit de 1984 sont-elles aussi à blâmer? On l'aura compris, je crois que tout ceci est avant tout le symptôme de dynamiques plus radicales. Je me contenterai donc ici de quelques éléments d'étonnement liés à ma (dé)-formation professionnelle qui m'inclineraient à penser que le problème mériterait, peut-être, d'être réinterrogé et reformulé. Pas pour plaider pour un retour vers la situation antérieure : je me sens encore trop jeune pour regretter, et trop âgé pour ne pas en avoir vu les possibles dérives. Non, plus modestement, pour contribuer à ce qu'en ces domaines qui me sont si chers, la connaissance et la culture, on ne confonde pas, une fois de plus, vitesse et précipitation.

D’abord, je vois dans l'évolution vers toujours davantage d'autonomie des universités et de course à l'excellence une logique qui tend à faire perdre son indépendance à l'universitaire - était-il un être plus autonome pré-LRU, pré-LPR ? - puisqu'on le transforme désormais définitivement en enseignant-chercheur-administratif. Une telle logique modifie la profession que j'avais choisie à l'origine et à laquelle j'associais l'idée d'une liberté qui valait bien quelques sacrifices, au moins à court terme. Parce que, puisqu'il faut chercher, alors il faudrait trouver. Parce que, puisqu'il faut enseigner, alors il faudrait diffuser. Parce que, puisqu'il faut diriger, alors il faudrait manager. On notera que tout ceci fait furieusement penser à la métaphore médicale de la perfusion : on la remplirait par les «trouvailles » de la recherche, on en diffuserait les résultats en " intraveineuse " par l'enseignement, le " médecin-surveillant » veillant à ce que le tube coule au bon rythme... Logiquement, on a postulé que la question critique est celle de l'incitation / sanction, et on a pris, à tort, l'image d'une université qui deviendrait une entreprise pour qualifier cette évolution - et ce alors même qu'il n'y a pas une forme unique d'organisation des entreprises ! II me semble donc important de rappeler quelques évidences gestionnaires et managériales qu'il serait bon d'opposer de temps à autre, et que je vois trop rarement formulées dans 
un ensemble cohérent. Ceci, peut-être par absence de connaissance en profondeur par les universitaires non familiers des sciences de gestion et du management de la manière dont sont organisées les entreprises. En tout cas celles qui ne sont pas obsédées par la seule maîtrise des coûts mais qui s'interrogent aussi sur ce que signifie construire l'avenir.

Commençons par la question de l'incitation / sanction, positive comme négative. Dans des activités par définition immatérielles, la question est toujours délicate. Les universitaires sont comme les chirurgiens, comme les juges et, finalement, comme tous les professionnels : du personnel de front office dont on sait l'impossibilité de les tenir réellement pour responsables des résultats alors même qu'ils ne maîtrisent pas l'état d'arrivée des clients ou des usagers. Dans leur domaine, immatériel, rien ne se possède, la connaissance est tout sauf un patrimoine susceptible d'être transféré... et ce même si le PowerPoint a été bien conçu. Comment, alors, vouloir les engager sur des objectifs et des résultats ? Un avocat, un chirurgien, un juge accepteraient-ils autre chose que l'obligation de moyens ? Dans tous les domaines qui ne s'exercent que par des professions, la problématique est celle de l'expertise et de la culture du diagnostic. Ceci ne s'acquiert qu'avec du temps, de la patience, des essais et des erreurs. Les apprentissages peuvent être naturellement plus rapides chez certains. Certains feront aussi partie des plus grands chirurgiens alors que d'autres seront d'abord d'excellents médecins généralistes...

Ceux qui connaissent un peu Henry Mintzberg savent cependant pourquoi, en aucun cas, on ne peut raisonnablement croire possible de standardiser les résultats des uns comme des autres, puisque seules leurs qualifications peuvent l'être, et le " clan " les réguler (Chatelain-Ponroy et Deville, 2020). La conséquence est immédiate : on ne saurait défendre sérieusement l'idée que l'universitaire qui publierait trop peu - ou qui ne serait pas suffisamment malin politiquement pour négocier quelques décharges administratives - devrait enseigner davantage. Ceci selon la logique surprenante qui pourrait amener à faire opérer plus un chirurgien, faire plaider plus un avocat, faire juger plus un juge... au motif qu'ils ne seraient pas suffisamment bons experts dans leurs domaines respectifs. Voilà comment on peut en venir finalement à apporter à un problème la pire des solutions faute d'avoir décelé la nature réelle de la maladie.

Poursuivons sur la question de la performance car les questions sont nombreuses et pas sans lien avec les points précédents. Qui évalue les évaluateurs ? La publication scientifique n'est-elle pas aussi une industrie à la Michael Porter avec ses rivalités concurrentielles à analyser, ses menaces de nouveaux entrants à prendre en compte, ses pouvoirs de négociation à diagnostiquer... et ses barrières à l'entrée qui peuvent la rendre très profitable pour les acteurs en place ? Autrement dit : vouloir s'intégrer à la grande danse de la publication scientifique internationale, pour mieux lutter dans la compétition, grimper dans les classements dits sérieux, n'est-ce pas aussi accepter de sauter par-dessus des barrières à l'entrée uniformisatrices, l'anglais, bien sûr, mais aussi circuler de train(s) en avion(s) pour participer aux congrès internationaux où il faut être pour envisager de commencer à exister, sans parler de l'impératif de traiter de questions d'abord dans l'air du temps?

J'ose poser la question : pour prendre le langage de l'économiste, tout cela ne fait-il pas, quand même, beaucoup de coûts de transaction à la Oliver Williamson ? Le coût d'opportunité n'est-il pas trop élevé ? L'investissement ne pourrait-il être 
mieux employé à lire autre chose, à prendre le temps d'écrire pour savoir ce que l'on pense, à profiter des nouvelles technologies pour partager avec le plus grand nombre des questions que l'on essaierait de renouveler aussi pour alimenter la conversation démocratique ? Pis, nos communautés académiques à l'étranger attendentelles d'abord d'être singées? Ne désirent-elles pas plutôt une pensée française qui serait à la hauteur de sa réputation ? Outre nos médailles Fields et nos prix Nobel, on oublie trop souvent que les disciplines des humanités ont aussi produit les auteurs parmi les plus cités au monde : les Pierre Bourdieu, Gilles Deleuze, Jacques Derrida, Michel Foucault, Edgar Morin, etc. On imagine d'ailleurs mal ces grands noms avoir toléré de se voir fixer des objectifs par des managers férus de seuls résultats... D’aucuns, mal intentionnés peut-être, verraient peut-être un lien entre le déclassement relatif des disciplines qui s'intéressent aux Humanités et la logique de performance en recherche : il est vrai qu'en ce domaine des Humanités, recherche et enseignement ont toujours été vues comme faisant système.

Continuons. Comment pourrait-on juger les présidents d'université et leurs équipes seuls responsables de la gestion des talents ? Une université, comme un hôpital, comme un tribunal, ne sera jamais propriétaire des dits talents : dans le domaine de l'immatériel, ils n'appartiennent jamais qu'aux individus qui la composent et acceptent (ou non) d'y apporter leur concours actif. Au mieux, une université ne peut donc être dotée que d'un patrimoine matériel, certes à entretenir (d'où l'éternelle question de la dévolution immobilière...), mais en aucun cas on ne saurait importer des recettes en provenance d'un monde où les entreprises gèrent d'abord des " produits ". La connaissance que porte les corps des universitaires et, plus généralement, le monde scientifique ne connaîtra jamais les frontières ni d'une université, ni d'une école, ni d'aucune institution; parce qu'au-delà des disciplines qui séparent les uns et les autres, les institutions d'enseignement, a fortiori dans le supérieur, sont toutes d'immenses bureaucraties professionnelles. Le prix Nobel Hayek (1945) - qui n'était pas un affreux collectiviste - l'a écrit et théorisé : la connaissance ne peut être mobilisée sans l'active coopération de ceux qui la détiennent. II serait bon de s'en souvenir en nos domaines où la tentation pourrait être grande d'assimiler, à tort, l'universitaire à un enseignant-chercheur-administratif qui deviendrait une sorte de prestataire de services dans ces trois domaines. Dans une industrie promise dès lors, comme les business schools, à la grande ubérisation (Albouy, 2019).

Dans l'univers immatériel de la connaissance, seules les qualifications peuvent être standardisées, et jamais autrement que par des pairs soucieux d'avancées et de progrès, pas de repos sur les acquis. C'est aussi parce que l'immatériel n'est pas matériel, que rien ne se décrète, que l'incitation et l'alchimie ne peuvent être que complexes, que l'innovation supporte mal la dynamique de compétition. Joseph Schumpeter notait d'ailleurs il y a un siècle ce point : l'innovation appelle des situations de stabilité à l'abri de la concurrence ? L'innovation a donc besoin de slacks, de ces formes de gaspillage qui hérissaient tant les managers quantophrènes du siècle dernier? L'innovation, la vraie, a besoin de pouvoir se défier des frontières telles qu'elles ont pu s'instituer, de création et de partage de connaissances, de capitalisation sur des savoirs disciplinaires distincts pour les faire dialoguer... Tout ceci, tous ces enseignements, il semble que les réformes successives du paysage universitaire français n'en ait pas tenu compte, ou, en tout cas, bien insuffisamment. 
Plus de concurrence, plus de compétition ne saurait être le seul stimulant, sauf à engendrer des dérives que l'on connaît déjà trop : travail sur les sujets fashion, recours privilégié aux grands échantillons et aux méthodes nomothéthiques qui sont les plus productives en termes de résultats; perte de vue des implication sociales et sociétales des produits de recherche pour ne plus considérer que les implications... for the research. Ce sont là de réels dangers. Alors que l'on sait suffisamment que l'obsession de la maximisation du ratio sur lequel on est jugé conduit parfois à prendre des risques, à être tenté par la triche, juste une fois, comme ça, puis de temps en temps, parfois... Au risque de se réveiller ensuite un peu tard et de faire le constat que les carrosseries des publications étoilées masquaient quelques vices cachés (plagiats, échantillons biaisés, etc.). L'actualité en fournit régulièrement des exemples. Ils iront croissants. À l'image, sans doute, des scandales financiers ou industriels qui ont émaillé les années 2000, puisque les mêmes motifs produisent avec une grande régularité les mêmes punitions. Je note que - mais est-ce un hasard ? - que la réforme de la gouvernance des universités n'a jamais abordé la question du contre-pouvoir que pourrait exercer l'universitaire par sa pensée et son indépendance. II y a ici pourtant matière à dialogue fécond avec le monde du droit et de la justice (Laufer et Muller-Lagarde, 2017). J'y reviendrai.

Par-delà leur diversité, tous ceux qui font profession de penser et donc d'enseigner, de partager, d'améliorer leur expertise aiment, au-delà de tout, comprendre pour agir, agir pour comprendre. La réalité leur parvient alors toujours filtrée au travers de lunettes théoriques. Ceci donne à voir des symptômes, des possibles à tenter et pour ceci il est nécessaire de disposer de données, de vouloir rechercher des lois pour mieux s'en défier. Le diagnostic est en effet cet art des prescriptions possibles fondées sur des intentions scientifiques... sans assurance aucune, jamais, de ne pas faire erreur ou de ne pas échouer. On est à l'opposé de toute la logique sous-jacente aux évolutions de gouvernance des universités. Pis, on est à l'exacte antithèse de ce que font celles que l'on croit prendre pour modèles mais auxquelles je crains que l'on n'ait pas compris grand-chose : les pratiques concrètes des entreprises.

On a donc pris le risque d'importer ce contre quoi les meilleures recherches en management se battent tous les jours : l'illusion du contrôle pour le contrôleur, d'un côté ; la gestion des indicateurs par les contrôlés, de l'autre. Puis, bien évidemment, l'introduction de dynamiques compétitives pour dresser les égocentrismes les uns contre les autres. Le tout, dans un univers où les ressources se raréfient, où pour bénéficier de l'allocation desdites ressources, il faudra sans doute plus que jamais désormais savoir " grenouiller " politiquement aux bons endroits, court-circuiter les circuits formels, pratiquer la bonne vieille logique des "visiteurs du soir ". Autant d'énergies qui ne seront plus mises à penser, à se défier des évidences, à prendre la parole pour se risquer à avancer des discours autres.

Deux remarques pour terminer une réflexion qui pourrait occuper un ouvrage tant les sujets à aborder seraient nombreux - qu'il s'agisse des dynamiques de concentration actuellement à l'œuvre, des réformes organisationnelles en cours, de la compétition interuniversitaire mais aussi intra-universitaire entre chacune des disciplines qui tentent de lutter pour leur survie, etc. :

- La première remarque, c'est que je vois très bien la manière dont la quantophrénie à tous les niveaux des diverses tâches de nos enseignants-chercheurs- 
administratifs peut conduire à flirter avec ce que les Anglo-saxons appellent le burn-out. Je ne connais pas d'équivalent en français, si ce n'est l'épuisement mental. Il est un mal qui guette, je crois, tous les universitaires, à tous les stades de leur carrière et ce quel que soit le statut qui régit leur activité. Ce risque de surinvestissement a été parfaitement pointé, par exemple concernant le fameux publish or perish. Je note qu'en dépit de toutes les critiques, rien n'y fait : on voit mal pour quels motifs ce publish or perish n'aurait pas encore de beaux jours devant lui en recherche. J'en déduis que c'est donc l'actualité de la souffrance au travail qui est le devenir naturel des enseignants-chercheurs-administratifs. On mesure tous les jours, hélas, qu'elle commence à être la chose la mieux partagée du monde, et singulièrement dans les organisations où les salariés ne voient plus grand sens à leurs actions. Dans le cas des universitaires, ce serait au détriment de l'exercice d'une pensée et du travail à son apprentissage et à son évolution. Ce serait dramatique pour notre démocratie.

- La seconde remarque, c'est que ce qui est en jeu dans nos domaines, c'est le non-quantifiable, le non-mesurable, bref l'immatériel de la pensée, de sa création, de son partage, de son influence. Autant de choses qui n'en sont pas et sont bien peu rentables politiquement dans le court terme des campagnes électorales puisqu'elles ne sont évaluables que sur la très longue durée. Ceci n'empêche pas visiblement de parler quand même de résultats, de tenter malgré tout d'envoyer le signal du volontarisme en espérant en toucher les dividendes politiques. On oublie alors trois points très importants. D’abord, on incite à aller là où les résultats, précisément, sont bien davantage reconnus et récompensés - je constate ainsi avec effroi depuis plusieurs années que beaucoup de mes collègues, parmi les meilleurs, partent vers d'autres horizons. Ensuite, on incite ceux qui restent à gérer avant tout les indicateurs, et ce à tous les niveaux. Enfin, on assèche de la sorte une réelle conversation démocratique sur la place de la culture et de l'université dans la société comme, par exemple, dans une politique d'aménagement du territoire. Je me demande donc si la propension des choses que l'on a ainsi créée ne risque pas de nous amener toutes et tous, tout simplement, dans le mur (Le Breton-Miller et Miller, 2021).

\section{Pour un Conseil national... de la Gestion et du Management}

De ce qui précède, je déduis une conséquence : après vingt ans de transformations radicales que rien n'aura pu freiner, l'heure n'est plus à la discussion et à la négociation, elle est au réveil et au sursaut. Nous, universitaires, avons la chance, jusqu'à nouvel ordre, d'être rémunérés par la société pour penser et exercer nos les qualités d'expertise dès lors qu'elles sont congruentes avec nos compétences. Je suis convaincu qu'il faut donc à tous ceux qui sont impliqués dans ce que représente la culture universitaire - et qui dépasse naturellement les seules universités et intègre toutes les institutions d'enseignement et de recherche - être bien plus ambitieux dans leurs projets d'investigation et dans leur éthique de prise de parole.

Celle-ci n'est jamais sans risques dès lors qu'il s'agit de jouer pouvoir contre pouvoir. Ceci place l'université et des universitaires en bonne place aux côtés des hôpitaux ou des tribunaux, etc., autant d'institutions qui subissent depuis deux 
décennies des ondes de choc massives en provenance du pouvoir politique. Alors que la profession de l'universitaire est l'exercice de la pensée et de son partage, il faut sans relâche se saisir de ce moyen d'action qui est l'un des plus puissants : la prise de parole et la liberté d'expression. C'est pourquoi je plaide volontiers pour que les universitaires, de toutes les disciplines - et singulièrement la mienne revendiquent leur pouvoir d'indépendance, et ce alors même qu'ils savent en fin connaisseurs de Michel Foucault qu'une société sans relations de pouvoir ne peut être qu'une abstraction.

Qu'ils assument et prennent des risques, comme celui d'émettre des avis quand leur discipline est convoquée pour justifier des réformes. Qu'ils s'expriment lorsque des fortunes trop étonnantes pour être parfaitement honnêtes sont faites. Qu'ils osent exprimer leurs avis sur les actions des hyper-managers, notamment (surtout ?) quand ils sont présidents de la République ou premiers ministres. Qu'ils réfléchissent, aussi, pourquoi pas, à des sortes d'" examens du barreau managérial " alors que la responsabilité que vont assumer les managers qu'ils forment sont absolument considérables dans nos sociétés contemporaines. Ainsi, il n'est définitivement pas " normal " qu'on ne puisse s'installer comme médecin, avocat ou pilote de ligne sans que des aptitudes aient été certifiées ; alors qu' " en même temps ", on peut parfaitement présider aux destinées de plusieurs centaines de milliers de salariés sans avoir jamais lu le moindre travail de recherche de management... Cherchez donc l'erreur (Lorino et Mottis, 2020) !

C'est chose possible aujourd'hui de remporter des victoires politiques décisives pour la discipline. Des jurisprudences aussi retentissantes que celle concernant l'affaire Jérôme Kerviel contre la Société Générale (Ouriemmi et Gérard, 2017 ; Denis, 2018), ou encore dans l'affaire dite des "suicides " chez France Télécom (Denis, 2019), démontrent qu'il n'y a aucune fatalité à ce que des managers ne rendent jamais des comptes en se cachant éternellement derrière le fameux « rien su, rien vu, rien entendu ". Non, l'heure des comptes et des désenchantements stratégiques et managériaux doit sonner, et c'est à l'heure de cette montre que les chercheurs doivent désormais régler les pendules épistémologiques de leurs disciplines respectives (Fabbe-Costes et Sépari, 2020 ; Fulconis et Paché, 2020). Puisque les dirigeants et (top-)managers sont précisément rémunérés pour savoir, voir, entendre mieux que ceux qui gagnent (beaucoup) moins qu'eux.

Il est possible de prendre appui sur des jurisprudences civile ou pénale (Société Générale, France Télécom) pour justifier de faire valoir les singularités de notre discipline auprès des pouvoirs publics et de l'opinion. Puisque notre discipline travaille sur les conditions endogènes de création (ou de destruction) de la confiance dans l'action collective finalisée face à l'incertitude, pourquoi ne nous mettrions-nous pas dans la roue de nos collègues des disciplines juridiques pour obtenir la création d'un Conseil national du Droit ? Pourquoi serait-il impossible d'envisager un Conseil national de la Gestion et du Management?

Ce serait une reconnaissance naturelle au plan épistémologique, tant à l'évidence le droit, le judiciaire, le management, le politique ont aujourd'hui parties et destins liés (Laufer et Muller-Lagarde, 2017; Hatchuel, 2019 ; Laufer, 2020). Les récentes mises en examen d'entreprises telles que Renault, Volkswagen et Citroën dans le scandale des fraudes anti-pollution (ainsi que de multiples responsables et dirigeants) démontrent en tout cas, si besoin était, qu'il n'est plus possible pour 
les communautés de recherche en management de rester en dehors de ces débats et de préférer continuer l'academic business de la publication as usual quand tout pourtant, autour d'elles, s'effondre.

\section{Conclusion}

Pour conclure, (dé)formé par ma spécialité, la stratégie, je suis convaincu que plus les dynamiques hétéronomes sont cousues de fil blanc, plus elles suscitent paradoxalement de saines réactions. Je suis certain que, toujours, on peut toujours (se) jouer de la contrainte et en profiter pour réaffirmer son identité et sa singularité. En un mot, son indépendance. Dans le cas des universitaires, l'indépendance va de pair - mais pour combien de temps encore ? (Paché, 2021b) - avec une responsabilité : être des maîtres de conférences ou des professeurs DES universités, du fait du statut que la société nous fait l'honneur de nous attribuer. De cette indépendance qui seule garantit la valeur de l'expertise, nous devons être digne, autant que nous le pouvons. Je continuerai, autant que je le pourrai, à tenter de le rester. Certains y verront peut-être de la naïveté ; j'en fais une question d'honneur. Cet honneur qui est, dans le domaine de la culture et de la connaissance, la plus puissante des incitations à continuer. Cet honneur qui vient, d'abord, des exemples auxquels on adhère, de l'inspiration léguée par ceux qui nous ont précédés. Cet honneur qui nourrit la passion, si importante dans notre profession; cette passion "long run » qu'aucune satisfaction d'intérêt « short term » ne pourra ni ne devra jamais remplacer. Du moins, je persiste à l'espérer encore. 
Explorer 



\title{
Un panorama de la face sombre de la motivation de service public
}

\author{
Effets inattendus \\ sur les attitudes et comportements individuels
}

François Grima et Irène GeOrgescu

Présentée comme " an individual's predisposition to respond to motives grounded primarily or uniquely in public institutions and organizations " (Perry et Wise, 1990), la motivation de service public (MSP) a fait l'objet de multiples travaux. Elle se définit comme un concept multidimensionnel. Quatre dimensions se dégagent : l'attrait pour l'élaboration des politiques publiques; l'attachement à l'intérêt général ; la compassion; et l'abnégation. La première traduit le goût pour la participation à la prise de décision publique, la deuxième montre l'importance du bien commun et du sens du devoir pour l'individu, la troisième souligne l'ouverture de la personne, son empathie à la situation de personnes peu privilégiées, et enfin la dernière exprime la volonté de sacrifier ses propres intérêts pour le bénéfice d'autrui. Jusqu'à une période récente, un consensus se dégageait pour décrire la MSP uniquement comme une source de comportements et d'attitudes positifs, tant dans la sphère professionnelle qu'en dehors. Elle est associée positivement dans la première sphère à une satisfaction dans l'emploi et à un engagement organisationnel (Crewson, 1997; Vandenabeele, 2009), alors que dans la seconde, elle rime avec engagement civique et politique (Ritz et al., 2016).

Sur cette base, la MSP apparaît comme un élément essentiel de la gestion des ressources humaines dans la fonction publique. Face à des situations marquées par l'ambiguïté de rôle, des demandes émotionnelles fortes source de questionnement de leur bien-être (Maslach et al., 2001), la MSP donnerait une énergie aux fonctionnaires pour gérer ces situations difficiles. Leur empathie, comme leur abnégation, devraient leur permettre de faire face à la confrontation de publics en difficulté sans que cela ne rime avec une quelconque forme de découragement. Leur engagement dans des actions de service public renforcerait leur capacité à faire face à ces situations difficiles. La MSP permettrait une meilleure performance (Ritz et al., 2016), et 
minimiserait l'absentéisme (Grant, 2008). En sus, Vandenabeele (2014) suggère que la MSP amène les fonctionnaires à être moins sensibles à une valorisation extrinsèque, comme une rémunération élevée, et à valoriser au contraire des dimensions intrinsèques de la motivation.

Des travaux récents sont venus questionner cette vision optimiste de la MSP. Ils suggèrent qu'elle pourrait avoir une face obscure (dark side). Dans un travail de synthèse, Schott et Ritz (2018) soulignent que cet intérêt pour cet aspect de la MSP est récent. Les premiers travaux empiriques ne datent que de 2012 (Giauque et al., 2012). Le but du chapitre est de mettre en lumière cette face obscure de la MSP en valorisant les travaux théoriques récents afin d'en poser une compréhension plus équilibrée et réaliste. Plus encore, nous cherchons ici à montrer que le nouveau regard porté sur la MSP entre en résonance avec l'actualité des évolutions dans le travail vécu par les fonctionnaires, incluant des enseignants-chercheurs à l'université, et ouvre au débat sur le management de la gestion des ressources humaines dans ce champ.

\section{Face sombre de la MSP : une prise de conscience progressive}

Comme nous l'indiquions précédemment, la littérature sur les conséquences négatives de la MSP reste récente. Cependant, il est possible de distinguer une évolution dans la dynamique d'analyse en faisant référence à trois temps.

\section{Premier temps : un questionnement de l'automaticité des effets positifs}

À un premier niveau d'analyse, jusqu'ici, des travaux contestent l'existence de liens affirmés entre la MSP et des attitudes ou des comportements décrivant une relation systématiquement positive entre le fonctionnaire et son organisation (ou son travail). C'est le cas de la relation entre la MSP et la satisfaction dans l'emploi (Lewis et Frank, 2002 ; Moynihan et Pandey, 2007). Giauque et al. (2012) suggèrent que la MSP est liée à une satisfaction qualifiée de " résignée ", qu'ils définissent comme une satisfaction personnelle associée à un désintérêt pour le travail s'expliquant par un environnement professionnel dans la fonction publique marqué par des contraintes et de la frustration. En d'autres termes, la MSP ne préfigure pas de manière systématique une satisfaction du fonctionnaire envers son organisation. II s'agit alors d'une première limite des effets positifs de la MSP venant nuancer l'optimisme suggéré par les précédents travaux sur le thème.

\section{Deuxième temps : l'émergence de conséquences négatives}

À un deuxième niveau, d'autres recherches sont venues pointer, non plus des remises en question des liens sur lesquels se fonde une vision positive de la MSP, mais les conséquences négatives du concept. Autrement dit, la MSP peut s'accompagner d'effets négatifs, voire inattendus, sur les individus, et les organisations 
plus largement. Dans une perspective individuelle, les relations de l'individu sont questionnées. Ainsi, Quratulain et Kahn (2015) suggèrent que la satisfaction résignée conduit le fonctionnaire à se désengager de son travail, voire à envisager de quitter la fonction publique. Jensen et al. (2019) proposent alors d'inclure dans la réflexion la notion du temps. Cela amène à reconsidérer fondamentalement les effets de la MSP. Si, à court terme, elle est synonyme d'un moindre absentéisme au travail tant le fonctionnaire est engagé dans son travail, à moyen et à long terme, la MSP est synonyme de dégradation de son état de santé. En ne prenant pas en compte sa santé, en la faisant passer au second plan après son travail, porteur de sens pour lui, il se met en danger à terme (Van Loon et al., 2015).

Un chemin argumentatif se fait jour ici articulant le temps et le contexte comme facteurs influant l'impact de la MSP sur la relation au travail. La nature du travail paraît jouer ici un rôle important. Les fonctionnaires avec un haut niveau de MSP se perçoivent obligés de rester au travail, quel que soit leur état de santé. Une telle perception d'obligation de se donner à son travail est d'autant plus forte que le fonctionnaire perçoit une situation difficile pour ceux qui pâtiraient de son absence. Ne pas pouvoir réaliser son travail aussi bien qu'il souhaiterait le faire débouche sur une élévation de son stress (Giauque et al., 2012). Le fonctionnaire se retrouve dans un cercle vicieux. Percevant une obligation morale à travailler pour ne pas mettre en danger des tiers, qu'il perçoit comme étant dans une situation difficile, il ne s'économise pas au travail, quel que soit les signes pouvant révéler une dégradation de sa santé physique ou mentale. Si ceci conduit à limiter l'absentéisme à court terme, une logique entraînant le présentéisme et l'absentéisme à long terme (Koumenta, 2015). La MSP ne s'accompagne pas uniquement de stress, mais elle peut donner lieu également à un burn-out (Liu et al., 2015). En effet, dès lors, le rôle de l'impact sociétal, tel qu'il est compris et intériorisé par le fonctionnaire, paraît être un facteur non négligeable pouvant expliquer les conséquences négatives de la MSP telles que le stress et le burn-out (Van Loon et al., 2015).

Comme on le voit avec ces différentes recherches, il apparaît que l'impact de la MSP sur les relations avec son organisation, son travail et sa santé doivent être repensées. Comme le rappellent plusieurs auteurs, les salariés les plus zélés sont souvent ceux qui ont le plus souvent à subir des conséquences graves au travail de ce sur-engagement (Demetrovics et Griffiths, 2012). On se retrouve ici dans le cas de l'application de la théorie des ressources où le collaborateur est confronté à une situation de perception de demandes de son travail élevées alors qu'il a peu de ressources (Bakker et Demerouti, 2007). Ritz et al. (2016) proposent une troisième voie de possible d'expression de la face obscure. Il s'agit de souligner que lorsque le fonctionnaire a une forte MSP, il a des attentes très élevées de réalisation dans le travail. L'impossibilité d'atteindre de tels objectifs peut conduire un individu à réaliser des actes de fraude au prétexte que son altruisme, fondé sur la satisfaction des intérêts de personnes qu'il perçoit en situation difficile, lui permet de s'exonérer de tout cadre moral. Ripoll et Schott (2022) confirment récemment cette perspective. 
Troisième temps : vers des propositions de soubassements théoriques

Schott et Ritz (2018) précisent qu'au-delà de la diversité des grilles d'analyse des effets négatifs de la MSP, il paraît pertinent de mettre en perspective la mauvaise adéquation entre l'individu et l'organisation (Kristof, 1996). Plus précisément, l'inadéquation peut concerner une personne et son organisation ou encore la personne et son emploi. Pour autant, la littérature ne décrit pas comment ces inadéquations entre ces éléments débouchent sur des conséquences négatives et comment potentiellement une adéquation peut, elle aussi, engendrer des conséquences négatives. Au-delà, ils précisent que la littérature s'est concentrée sur les conséquences attitudinales et a délaissé la compréhension de cet impact négatif de la MSP sur les comportements. Face à ce hiatus, entre un constat d'impacts négatifs importants de la MSP sur les individus et une méconnaissance de la construction de ses conséquences, Schott et Ritz (2018) proposent de mobiliser plusieurs cadres théoriques en distinguant ceux fondés sur une approche en termes de mauvaise adéquation entre la personne et son organisation de celle, au contraire, reposant sur une adéquation entre les deux. Deux grilles théoriques sont regroupées dans le premier ensemble :

- La première grille est celle de la théorie de l'identité. Vandenabeele (2007) suggère que la MSP peut être comprise comme l'expression d'une identité de service public. S'appuyant sur le processus de vérification de l'identité décrit par Burke et Stets (2009), les auteurs soulignent que la MSP place les fonctionnaires dans une dynamique d'engagement d'autant plus forte dans leur travail que le contexte dans lequel ils le réalisent est contraire à cette identité. Un cercle vicieux se développe : plus le fonctionnaire tente de valider sa MSP dans son travail et rencontre des difficultés, plus il s'engage fortement dans le travail, au point de mettre sa santé en jeu. Les auteurs suggèrent que certaines dimensions de la MSP peuvent être plus sensibles que d'autres à un tel enchaînement. La compassion, qui traduit un état empathique et d'identification avec des personnes en situation de faiblesse, leur apparaît comme particulièrement affectée.

- La seconde grille est celle du contrat psychologique. Il apparaît ici que les individus sont dans une recherche de validation des engagements mutuels les unissant avec leur organisation. Tout vécu de non-respect de cette réciprocité anticipée conduit à un vécu de rupture du contrat psychologique que Giauque et al. (2012) analysent en termes de satisfaction résignée. Les auteurs l'envisagent comme une forme de stratégie de coping passif à une situation stressante. La mobilisation de ce cadre théorique conduit à appréhender le lien entre MSP et la satisfaction au travail dans une logique de réciprocité entre l'État employeur et le fonctionnaire. Selon cette logique, lorsque le fonctionnaire estime que la réciprocité est faible, il va être dès lors enclin à attendre moins de son travail, et ainsi être moins satisfait. Schott et Ritz (2018) suggèrent une autre explication en soulignant que la théorie du contrat psychologique rime avec prédictibilité des comportements. Or, lorsque le fonctionnaire perçoit un contexte organisationnel qui n'est pas conforme à ses anticipations, il éprouve du stress et développe une intention de partir. L'absence de maîtrise de la situation, pensée comme contrôlable et prévisible, est source de tensions. La personne est placée dans une situation où elle ne peut que constater son impuissance à faire face à une situation où, au contraire, elle devait être en 
capacité d'agir. Un tel constat douloureux d'incapacité à agir rime alors avec le développement d'une adaptation fondée sur une stratégie d'adaptation passive.

Là encore, toutes les dimensions de la MSP ne sont pas affectées de manière équivalente. La dimension principale du MSP affectée est celle de l'engagement pour les valeurs de service public car elle s'appuie sur une conception élargie du travail du fonctionnaire. En revanche, les facettes de l'abnégation et de la compassion semblent moins affectées car elles témoignent de l'aide que le fonctionnaire est prêt à apporter à des publics perçus en difficulté. Pour la perspective valorisant une adéquation entre la personne et son organisation, Schott et Ritz (2017) retiennent un seul cadre théorique : le modèle ASA (attraction sélection et attrition) (Schneider et al., 1995). Ils précisent que le modèle valorise - sur le moyen terme - une homogénéité de conception du travail dans une organisation, du moins tant que ne reste en son sein les individus ayant satisfait des conditions de travail. Schneider et al. (1995) soulignent la difficulté des membres d'une organisation à accepter des changements dans leur environnement et pointent les limites d'une pensée de groupe (groupthink). Les individus restent fidèles à leur engagement initial qui les a conduits à devenir membre de leur organisation; ils deviennent inflexibles à l'évolution de leur environnement. Repris dans le cas de la MSP, cette grille d'analyse a toute sa pertinence, tant l'âge moyen des fonctionnaires est élevé et les changements dans leur environnement professionnel sont importants avec l'émergence, notamment, de pratiques issues du new public management. Ainsi, les évolutions, plus ou moins récentes, du secteur public viennent questionner la manière dont les fonctionnaires perçoivent leur travail.

Au-delà de ces propositions théoriques, Schott et Ritz (2018) indiquent l'existence d'une autre zone d'ombre dans la compréhension des conséquences négatives de la MSP : sa dimension comportementale. Les auteurs font référence à la « noble cause corruption ". Une survalorisation de la dimension compassion de la MSP peut conduire le fonctionnaire à questionner sa neutralité et à développer des comportements non éthiques au profit de publics qu'ils perçoivent en difficulté. Une telle dérive ne doit pas être confondue avec la corruption où le fonctionnaire cherche à tirer un profit de ses actes délictueux. Ici, la formule fameuse " la fin justifie les moyens " s'applique pleinement. L'empirie fondant cet enchaînement est celui de la police où des policiers ne respectent pas la loi pour essayer de faire accuser des criminels. Plus précisément, dans ce dernier cas, les policiers vont contourner les procédures et la loi afin de protéger une population face à un individu qu'ils qualifient de menaçant. Plus radicalement, Adams et al. (2006) décrivent un administrative evil pour désigner un aveuglement pouvant toucher des fonctionnaires dans l'exercice de leur travail. II s'agit ici d'une justification morale qui entre en résonance avec la théorie du désengagement de Bandura (2000). Le comportement déviant est repensé mais sera légitimé et justifié par les individus; la déviance apparaît comme justifiée moralement.

Les éléments précités conduisent à repenser les diverses facettes du MSP et leurs effets inattendus, voire contre-intuitifs. En effet, la compassion semble être une dimension pouvant amener à ce type de dérive comportementale. II en va de même pour la dimension d'adhésion aux valeurs publiques. Pour les deux autres dimensions (abnégation et attraction au service public), la question de leurs liens avec les 
pratiques comportementales déviantes se pose moins. On retrouve cette dynamique avec le concept de prosocial rule-breaking compris comme une déviation institutionnelle visant à satisfaire les intérêts collectifs d'un groupe perçu comme en situation de fragilité. Morrison (2006) le définit comme

any instance where an employee intentionally violates a formal organizational policy, regulation or prohibition with the primary intention of promoting the welfare of the organization or one of its stakeholders.

Face à des règles qu'ils perçoivent comme peu compatibles avec les intérêts de groupes en situation difficile, les fonctionnaires peuvent être tentés de développer des comportements de prosocial rule-breaking (Borry, 2017). Autrement dit, des comportements peu appropriés peuvent être remarqués chez un fonctionnaire dont l'intention est d'œuvrer pour l'intérêt du collectif ou le bien de l'organisation.

\section{Une compréhension contextualisée des effets de la face sombre de la MSP : le cas de bureaucraties professionnelles}

Si le travail de Schott et Ritz (2018) constitue une étape indéniable dans la construction d'un processus d'accumulation de la connaissance scientifique autour des conséquences négatives de la MSP, des travaux récents, notamment d'Awan et al. (2020), approfondissent la réflexion. Ils soulignent que la compréhension de la MSP nécessite une approche contextualisée. Deux dimensions apparaissent ici comme essentielles :

- La première dimension est le contexte législatif. Les auteurs mettent en avant la différence entre les pays de common law et ceux de civil law. Alors que les premiers bâtissent des lois pour limiter le pouvoir de l'État, les seconds les construisent pour l'affirmer. Awan et al. (2020) précisent que l'essentiel des travaux empiriques sur la MSP se sont développés dans des pays de common law. C'est pourquoi ce sont principalement les conséquences positives qui furent mises en avant tant ce contexte juridique est synonyme de valorisation de liens entre la MSP et des comportements positifs (comme les comportements citoyens).

- La seconde dimension est l'existence de la corruption. Ici, de l'aveu même des auteurs les résultats avancés se fondant sur une méta analyse de la littérature sont contre intuitifs. Ils suggèrent que la MSP ne voit pas sa relation se dégrader avec la satisfaction au travail dans un contexte de corruption. Cette situation pourrait s'expliquer pour la perception d'une nécessité d'un travail de qualité dans un contexte où il est rare. La perception d'un réel apport différentiel est synonyme de satisfaction au travail. En revanche, la corruption engendre une détérioration de l'engagement organisationnel comme des comportements citoyens. Weißmüller et al. (2022) suggèrent que la contextualisation de la compréhension des conséquences négatives de la MSP doit intégrer les caractéristiques du travail, comme l'autonomie dans la réalisation ou les comportements des supérieurs et des collègues.

Les travaux conduits en France, mais également à l'international, illustrent l'importance du contexte dans l'analyse de la face sombre du MSP à deux niveaux : attitudinal et comportemental. En effet, dans les bureaucraties professionnelles, la mise en œuvre du new public management met en lumière des tensions autour de la 
MSP. Dans le domaine attitudinal, le new public management s'accompagne d'effets délétères sur le travail et la santé au travail des fonctionnaires. Si la volonté affichée des Pouvoirs publics est d'accroître la performance et la transparence des organisations publiques, toute structure bureaucratique, une fois ancrée dans une organisation est particulièrement complexe à faire évoluer. La bureaucratie est caractérisée, notamment, par une gestion qui se base sur les écrits, des structures, des acteurs chargés de les conserver et d'assurer leur régularité. L'action, dominée par les règles, par la standardisation des procédures, vise à renforcer le rationalisme : « le contrôle et à la régulation interne indiquent déjà des risques de technocratisation des systèmes de gestion, liés à l'hyper-rationalisme " (Bezes, 2005). Le développement du new public management et, simultanément, de la bureaucratie, de l'État, des normes et du capitalisme modernes (Le Galès et Scott, 2008), soulignent comment ce dernier, par la déstabilisation des institutions et la création de nouveaux systèmes de sanctions et récompenses, rend prévisibles les comportements individuels et collectifs.

La mise en place de nouvelles réglementations peut s'accompagner d'une surcharge de travail pour les fonctionnaires (Diefenbach, 2009). Répondre aux injonctions de performance, dans un contexte d'accroissement des procédures, vient intensifier le travail des fonctionnaires et des professionnels. En contexte de new public management, il est possible d'observer un accroissement du stress (Kirkpatrick et al., 2005), ou encore sur l'insatisfaction au travail (Mikkelsen et al., 2000). Ces développements retrouvent les conclusions des travaux précédemment mentionnés sur la possibilité de lier MSP et conséquences négatives pour la relation avec le travail et l'organisation. II apparaît que les orientations portées par le MSP constituent un climat propice à cet enchaînement négatif autour de la MSP. Par ailleurs, l'accroissement des procédures vient menacer le travail réel des fonctionnaires, déjà éloigné du travail prescrit (Clot, 2010), ou encore affecter les collectifs de travail (Morley, 2005). Le new public management participe au phénomène de dégradation des conditions de travail en agissant sur le burn-out par l'intermédiaire de la charge de travail et de l'augmentation du climat de violences psychologiques (Abord de Chatillon et Desmarais, 2012).

En contexte universitaire, au Royaume-Uni, les évolutions de nature du travail universitaire, marquées par exemple par les évaluations de la qualité de l'enseignement par les étudiants et l'évaluation de la recherche, permettent aux gestionnaires d'accroître leur pouvoir et leur contrôle sur les professionnels que sont les enseignants-chercheurs. Ainsi, la culture de l'étudiant " client » se déploie progressivement dans les universités (Knights et al., 1994). Elle est couplée aux changements socio-économiques, pour exposer de fait les organisations du secteur public à la « discipline du marché " (Willmott, 1995). Il est alors observé que l'intensification du processus de travail universitaire, exacerbé par la réduction de leur autonomie et des tentatives croissantes de contrôle et d'intensification de tous les aspects de leur travail, accroît le travail émotionnel des enseignants à l'université, le stress et le manque d'esprit d'équipe (Ogbonna et Harris, 2004). Motivés par une volonté de maintenir le service public et ses missions, certains fonctionnaires développent des comportements qualifiés, en première intention, de déviants. Précisons ce point important.

Comme la MSP le suggère, les individus travaillant dans le secteur public ont pour dessein de servir l'intérêt public sur la base de considérations éthiques (Houston, 2006). Le comportement prosocial, tel que l'engagement dans des 
activités de bénévolat, la participation à des activités caritatives (Clerkin et al., 2009), le comportement de citoyenneté organisationnelle (Kim, 2006), est associé à la MSP ; ils engendrent des comportements éthiques (Maesschalck et al., 2008). Néanmoins, la MSP dans le contexte d'une organisation publique peut conduire à des comportements déviants pour plusieurs motifs. Comme le rappellent plusieurs auteurs, dont Perry et Wise (1990), la MSP encourage les fonctionnaires à faire preuve de compassion envers les autres, et à sacrifier leurs intérêts personnels en faveur de l'intérêt public. Cet encouragement à sacrifier ses intérêts personnels en faveur de ceux de l'organisation peut donner lieu au développement de comportements non éthiques pro-organisationnels, définis comme toute action qui vise à promouvoir le fonctionnement efficace de l'organisation ou de ses membres et qui viole les valeurs sociétales fondamentales, les mœurs, la loi ou les normes de bonne conduite (Umphress et Bingham, 2011). La MSP encourage les employés à choisir la voie qui repose sur des valeurs fondées sur l'intégrité personnelle qui mettent l'accent sur la discrétion, la réflexion, la vertu et l'intuition en accord avec le sens personnel de la moralité (Stazyk et Davis, 2015). Une telle possibilité d'interpréter le comportement éthique de manière personnelle offre des opportunités pour des comportements non éthiques tournés vers l'intérêt de l'organisation publique dans la mesure où ils résultent d'une appréciation personnelle de l'éthique. Aussi, des comportements déviants au travail sont susceptibles de se développer, motivés par le secteur public.

Les réformes menées dans les organisations publiques ont eu pour effet de réduire les effectifs de la fonction publique et le niveau d'engagement envers l'éthique du service public. Par exemple, Miao et al. (2013) explorent les liens entre le leadership éthique et les comportements peu éthiques pro-organisationnels. Fondée sur une étude dans le secteur public en Chine, la recherche met en lumière un effet curviligne du leadership éthique et des comportements peu éthiques pro-organisationnels. Les auteurs soulignent le rôle joué par l'éthique et l'identification de l'individu dans la régulation des comportements peu éthiques : le contexte éthique dans lequel l'individu évolue peut contenir plus ou moins ces comportements. De même, dans le secteur public hospitalier, le développement de comportements d'optimisation du codage sont observés (Milcent, 2016). En jouant sur leurs marges de manœuvre, les professionnels de santé, s'engageant dans ces comportements énoncent, pour certains, leur motivation à défendre le service public hospitalier (Georgescu et al., 2018). Ainsi, bien qu'elle soit peu explorée, la relation entre la MSP et les résultats contre-productifs sont des pistes de recherche prometteuses pouvant être étudiées plus attentivement (Ritz et al., 2016).

\section{Conclusion}

Comme le suggèrent Vandenabeele et al. (2018), la MSP est loin d'être " un problème résolu ॥. La nature pluridimensionnelle du concept, comme l'influence de nombreuses sciences sociales dans sa définition (psychologie, sciences politiques, sociologie, économie, sciences de gestion et du management), contribuent à rendre délicate la compréhension tant des antécédents que des conséquences du concept. Plus encore, un hiatus émerge. D'un côté, le contexte opérationnel dans les organisations publiques rend la compréhension de la MSP essentielle pour optimiser les ressources humaines mises sous tension par une application difficile du new public 
management. De l'autre, une communauté scientifique s'est focalisée pendant de nombreuses années sur une grille de lecture ne valorisant qu'une approche optimiste de la MSP. Or, s'intéresser à la face sombre de la MSP contribue à enrichir le débat et surtout, selon nous, à répondre à l'objectif des sciences de gestion et du management : aider les organisations à optimiser leur fonctionnement.

Le présent chapitre souhaite avertir les décideurs publics que, loin de se limiter à des conséquences individuelles négatives, la MSP peut avoir des conséquences organisationnelles négatives. II convient ici de décaler le regard et de passer d'un niveau individuel à un niveau organisationnel. Schott et Ritz (2018) nous y invitent, en indiquant que des fonctionnaires épuisés, stressés, sont sources d'une faible qualité de service, voire d'un potentiel mécontentement de la population. En cela, I'un des objectifs premiers du new public management, qui est d'apporter un service à un usager client du service public, n'est pas atteint. Ceci nous permet de constater que les intérêts des fonctionnaires comme de l'État employeur sont alignés à moyen terme. II convient donc de relativiser une approche à court terme de la face sombre de la MSP qui voudrait que cette dernière soit uniquement négative pour les fonctionnaires à la santé dégradée, alors que l'État employeur bénéficierait d'un surcroît de travail à un coût réduit. En intégrant dans l'analyse une perspective de moyen et de long terme, il apparaît que l'État employeur doit penser sa légitimité sociale, notamment s'il conçoit son action en termes de prestation de services envers des usagers assimilés à des clients. Ne pas être à la hauteur de sa promesse à la population, c'est prendre le risque de voir se développer une perception d'hypocrisie organisationnelle dont les conséquences sociales pourraient être difficiles à gérer. 



\section{Gestion des terrains sensibles en sciences de gestion et du management}

François Grima et Olivier MeIER

À l'instar des disciplines des sciences sociales (Adler et Adler, 1993; Renzetti et Lee, 1993 ; Bouillon et al., 2005 ; Hennequin, 2012 ; Ayimpam et Bouju, 2015), les sciences de gestion et du management, adoptant notamment des approches qualitatives, sont aujourd'hui, particulièrement sensibles aux aspects éthiques et méthodologiques que soulève la gestion des terrains sensibles (Sachet-Milliat, 2009 ; Merle, 2010 ; Condomines et Hennequin, 2013). Ce type de terrains s'est fortement développé ces dernières années face à la mondialisation, à la généralisation des nouvelles technologies de communication et aux évolutions sociales et sociétales (mouvements de populations, affirmations culturelles) qui ont envahi la sphère publique, privée et, plus globalement, le monde de l'entreprise, en venant questionner un certain nombre de ses pratiques et comportements (Jan et al., 2019). La littérature sur les sujets sensibles pointe trois principales lacunes sur le plan conceptuel et méthodologique. La première concerne le terme même de "sensibilité » dont l'acceptation apparaît trop limitée et schématique. La seconde porte sur les groupes étudiés et leurs liens avec la question des terrains sensibles. Le troisième point porte sur les aspects méthodologiques et la place du chercheur dans le dispositif d'ensemble, compte tenu des défis émotionnels à gérer et de la nécessité de produire des résultats fiables.

Un aspect de la littérature méthodologique sur les sujets " sensibles " au cours de la dernière décennie concerne le manque d'intérêt persistant pour le développement de ce concept. Une grande partie de la littérature a tendance à prendre la notion de " sensibilité " comme non problématique ou de sens commun, ou tend à reposer sur la définition de Lee (1993) en tant que " recherche pouvant potentiellement constituer une menace importante pour ceux qui sont y ont été impliqués ». Les travaux originels de Lee (1993) abordent la question de la " sensibilité » comme un phénomène émergent et non comme une caractéristique inhérente entre le sujet et la recherche. De ce fait, une acceptation plus riche pourrait être de substituer la notion de " risque " à celle de " menace ", ouvrant ainsi la voie à d'autres analyses et interprétations (Dickson-Swift et al., 2008). En effet, la notion de " menace » 
peut, par certains côtés, apparaître comme étant trop mécaniste et psychologique. À ce jour, cependant, un travail de ce type reste à faire. Le besoin d'un développement conceptuel plus poussé est peut-être le plus perceptible en ce qui concerne la littérature sur les exigences émotionnelles. La question des défis émotionnels auxquels les chercheurs sont confrontés lorsqu'ils travail sur le terrain mériterait une attention plus grande.

De plus, de nombreux travaux sur des " sujets sensibles " se concentrent sur des groupes stigmatisés, défavorisés ou socialement ignorés d'une manière ou d'une autre. Cependant, des travaux mettent en évidence une lacune importante. En effet, force est de constater le manque de travaux sur les élites au pouvoir, sur des sujets concernant les méfaits des entreprises (comportement des dirigeants) ou sur le pouvoir de l'État, qui sont pourtant des domaines sensibles et délicats qui, à bien des égards, posent une série de difficultés pour les chercheurs. L'importance d'étudier "vers le haut " comme "vers le bas " reste un défi de longue date pour les chercheurs qu'il convient de traiter (Nader, 1972). Quelques conseils sur les questions en jeu pourraient par exemple provenir d'un travail sur la négociation des complexités politiques des sociétés.

En ce qui concerne les aspects méthodologiques, les chercheurs peuvent prendre trois grandes directions. Premièrement, les universitaires doivent éviter d'affirmer que la connaissance est totalement " neutre " d'un point de vue éthique, car peu de personnes en dehors du domaine académique partagent ce point de vue. La question de l'interprétation des résultats pour faire avancer sa propre cause reste posée (Sieber et Stanley, 1988). Deuxièmement, il est important que les chercheurs choisissent des procédures qui leur fournissent les moyens de répondre aux critiques et de protéger les résultats contre les attaques éventuelles (Channels, 1993), face aux risques d'exposition de leurs travaux au grand public. Enfin, troisièmement, les chercheurs doivent réagir de manière proactive à ce nouvel environnement pour s'assurer que les informations diffusées soient exactes et puissent permettre de faire évoluer la science.

\section{Définitions de terrains ou sujets sensibles : proposition d'une typologie}

Comme le note Condomines et Hennequin (2013), s'intéresser aux sujets sensibles, c'est se confronter au premier abord à une compréhension naturaliste de cette thématique, laissant aux chercheurs une grande liberté dans la définition retenue du concept. Pour autant, un examen plus approfondi de la littérature témoigne de l'existence de plusieurs travaux visant à définir le concept de sujet sensible en sciences de gestion et du management. La référence de base demeure celle de Sieber et Stanley (1988) qui énoncent que

socially sensitive research refers to studies in which there potential social consequences or implications, either directly for the participants in the research or for the class of individuals represented by the research.

Condomines et Hennequin (2013) rappellent que cette définition constitue une première tentative pour donner un contenu à un concept qui, jusqu'ici, se limitait 
à l'étude d'un tabou. Les auteurs soulignent que cette première compréhension est trop restrictive et déterministe tant elle oublie les éléments contextuels, sources de vulnérabilité. La définition de Sieber et Stanley (1988) a le mérite de replacer au cœur de la réflexion les personnes interrogées et d'interroger le chercheur par rapport à son objet et ses conséquences pour la société. Malgré ses mérites, cette définition fait l'objet de critiques.

Lee (1993) souligne l'absence de prise en compte des dimensions éthiques, méthodologiques et techniques liées à la réalisation de recherches sur des sujets sensibles. Il propose une nouvelle définition des sujets sensibles comme : "Research which potentially poses a substantial threat to those who are or have been involved in it ". Lee (1993) précise que les sujets sensibles peuvent concerner des sujets comme les comportements contraires à l'éthique ou scandaleux, la violence au travail, le harcèlement, la discrimination, l'utilisation de procédures dangereuses, les cas de " coming out " au travail ou de consommation de drogues illicites, et des comportements déviants dans l'organisation. Lee (1993) souligne qu'il convient de tenir compte dans la définition de facteurs contextuels qui peuvent rendre un sujet sensible dans un environnement particulier. Ainsi, la perspective de mener une thèse de doctorat sur " l'impact du terrorisme islamiste sur le modèle démocratique aux États-Unis : une lecture des attentats du 11 septembre " peut apparaître, chez les victimes et familles de victimes (2 977 personnes décédées) qui travaillaient dans le centre de Manhattan à New York, comme un sujet hautement sensible tant sur le plan émotionnel que psychologique. La prise en compte du contexte et de son impact social, sociétal ou psychologique sur les personnes concernées, constituent donc un élément essentiel de la gestion des recherches en milieu sensible.

Ces deux premières définitions ont ouvert la voie à la définition de Renzetti et Lee (1993) qui apparaît comme la référence dans le champ des sciences de gestion et du management. Les auteurs s'inscrivent dans le prolongement des travaux précédents, notamment celui de Lee (1993). Ils approfondissent la réflexion autour de la notion de menace potentielle. Ils identifient quatre circonstances qui conduisent à une intensification de cette menace. La première est lorsque la recherche s'introduit dans la vie privée des interviewés ou a pour objet des sujets mobilisant une expérience personnelle particulièrement privée ; ici, le chercheur est invité à intégrer dans ses analyses le caractère intrusif de sa démarche qui peut engendrer parmi les interviewés des sentiments négatifs comme la honte ou la peur. La seconde concerne les recherches s'attachant à comprendre des pratiques déviantes; ici, la divulgation de ces réalités cachées peut être synonymes de coûts élevés pour les interviewés tant la société peut chercher à les condamner pour ces pratiques illicites. La troisième menace souligne le poids que peut avoir sur les interviewés des résultats concernant les intérêts de personnes en position de pouvoir ; ici, les auteurs soulignent que les conséquences négatives peuvent être très élevées au point de concerner aussi le chercheur. Enfin, Renzetti et Lee (1993) suggèrent la menace potentielle constituée par l'étude de sujets perçus comme essentiels, sacrés par les interviewés. Leur capacité à accepter une relativisation de l'importance du sujet, voire une critique est très faible. Condomines et Hennequin (2013) voient en l'espèce un rappel du caractère tabou des sujets sensibles.

L'ensemble de ce travail terminologique traduit la volonté de la communauté scientifique de mettre au premier plan de la définition des sujets sensibles la 
protection des publics sensibles comme celle des chercheurs. Les travaux menés dans le milieu médical auprès de malades ou d'enfants retrouvent cette exigence éthique non discutable. La sensibilité du sujet est déterminée par la vulnérabilité des personnes interrogées. Cette dernière est définie par Peternelj-Taylor (2005) comme un construit complexe et multi dimensionnel, évolutif selon les croyances personnelles et les valeurs de la société où la recherche est menée. Kipnis (2001) propose cependant de ne pas faire de cette vulnérabilité un obstacle à la recherche lorsqu'il énonce à propos du concept qu'il ne devrait pas être vu comme un "flashing red light ordering researchers to stop, but rather as a cautionary signal, calling for proper safeguards ». On voit poindre ici une critique sur le poids pris dans le contexte anglosaxon par les comités éthiques qui se saisiraient de la vulnérabilité de certains publics pour en limiter l'étude. Or, Alexander et al. (2018) montrent, en s'appuyant sur les travaux d'autres chercheurs comme Biddle et al. (2013), que les bénéfices pour ces publics fragiles de participer à des recherches les concernant dépassent de loin les risques pris. Plus encore, les auteurs suggèrent que les coûts supportés ne durent pas ou sont d'un faible niveau face à la satisfaction de pouvoir témoigner qui, elle, s'inscrit dans le long terme.

Ces recherches récentes témoignent d'une inflexion dans la réflexion autour des sujets sensibles. Elles tendent à montrer que la protection des publics vulnérables s'est érigée en tabou. La communauté scientifique serait dans l'impossibilité de le dépasser, ce qui la conduirait à laisser dans l'ombre des problématiques humainement, socialement pertinentes. Beji-Bécheur et al. (2011) montrent dans leur travail sur l'ethnicité que la prise en charge d'un sujet sensible conduit les chercheurs à le construire en tant que tel comme sensible et à se construire une vision de leur responsabilité dans son traitement. On retrouve ici le constat posé par Condomines et Hennequin (2013) pour qui « le caractère sensible d'une recherche réside moins dans le thème traité que dans la relation entre ce sujet et le contexte social dans lequel l'étude est menée ".

De telles réflexions montrent que la recherche sur les sujets sensibles évolue d'une définition stricte fondée sur la vulnérabilité des participants vers une approche plus contingente où l'opinion des participants, comme celle des chercheurs, prend le pas sur des facteurs exogènes comme l'avis d'un comité éthique. La sensibilité d'un sujet apparaît donc plus fragile tant elle est affectée par la compréhension mouvante que peut en avoir un chercheur qui peut penser le sujet sensible au démarrage de son travail et réviser son jugement au fur et à mesure de sa découverte ou inversement. Condomines et Hennequin (2013) soulignent cependant que certains sujets comme la sexualité et la maladie sont par nature plus sensibles. Dans un article de synthèse des travaux menés ces dix dernières années sur les sujets sensibles, Lee et Lee (2012) invitent la communauté scientifique à ne pas se contenter de la définition du caractère sensible d'un sujet sur le seul critère de la menace potentielle. Pour les auteurs, Lee (1993) suggère que ce caractère est émergent et non propre à un sujet. Face aux limites inhérentes associées au terme "menace ", les auteurs proposent de lui substituer le terme risk, qui leur apparaît plus riche conceptuellement et plus flexible.

C'est en ce sens que notre compréhension du sujet sensible souhaite aller. Nous définirons le caractère sensible d'un sujet par les risques dont il est porteur pour les participants au sens large. Plus précisément, nous intégrons bien évidemment 
les sujets de la recherche mais aussi le ou les chercheurs menant le travail. En effet, alors que les travaux récents soulignent le travail entrepris par le chercheur autour du caractère sensible (Béji-Bécheur et al., 2011 ; Condomines et Hennequin, 2013), les différentes définitions existantes se focalisent sur les seuls participants. Une telle conception nous apparaît comme restrictive. La construction de l'objet de recherche comme sensible, la gestion de la relation avec l'environnement social proche, comme la communauté scientifique, ou plus éloigné à l'instar des médias, constituent des défis importants pour le chercheur. Ce dernier, comme ceux l'encadrant dans sa démarche (collègues, directeur ou directrice de thèse) et les diffuseurs (éditeurs de revues) constituent autant d'autres acteurs potentiellement impactés, menacés par une recherche sur un sujet sensible. Ne concevoir ce concept uniquement qu'à partir des personnes en constituant le terrain oublie la moitié de ceux réellement touchés par la démarche. Sans mettre sur le même plan les deux populations, ceux faisant la recherche et ceux en étant le sujet, les premiers prennent aussi des risques dans la démarche. On peut penser ici à l'absence de reconnaissance de la qualité du travail, des questionnements éthiques sur les raisons de ce choix de sujets, la volonté de régler des comptes personnels avec une population ou une institution particulière, etc.

Au-delà, et de manière plus iconoclaste, il semble pertinent, à l'image de l'invitation de Lee et Lee (2012), de nous interroger sur le biais présent dans la littérature faisant que la quasi-totalité des travaux sur les sujets sensibles ne concerne que des populations vulnérables. Sensibilité devient alors synonyme de vulnérabilité. Or, s'interroger sur les pratiques mafieuses de certains dirigeants, mettre à jour I'hypocrisie organisationnelle de certaines organisations (Brunsson, 1993, 2002 ; Dumez, 2016), constituent autant de sujets où, à la fois les participants (interviewés) comme les organisateurs de la recherche (chercheurs et diffuseurs), prennent des risques. À ce titre, selon Brunsson (1993), la séparation de l'organisation politique (qui créé de la légitimité vis-à-vis de l'environnement extérieur) et de l'organisation d'action (qui coordonne les actions informelles au sein de l'entreprise) est possible en cultivant l'hypocrisie organisationnelle permettant de contenter les intérêts des différents groupes présents dans et hors de l'organisation.

Sur la base de ces différents arguments, dans un souci d'élargir une compréhension étroite du sujet sensible, nous proposons une nouvelle définition du concept. Un sujet sensible est une recherche qui présente des risques pour ceux qui y sont impliqués tant comme participants que comme producteurs de connaissances. Cette définition va donc au-delà de la question de la vulnérabilité, terme selon nous trop restrictif, pour lui préférer celle de risque et de ses conséquences en matière de conduite de recherche. Elle présente l'avantage d'intégrer dans la réflexion, les questions d'ordre éthique mais également d'approfondir les questions de nature méthodologique (positionnement de la recherche, gestion du protocole, mode de collecte et de traitement, confidentialité des résultats, etc.).

En reprenant cette définition, nous proposons de recenser les types de terrains (situations) dans lesquels la relation entre le sujet et le contexte social peut être contrarié, en raison des menaces ou des risques qui pèsent sur l'ensemble des acteurs concernées par la recherche, qu'il s'agisse des cibles retenues ou du chercheur lui-même. En effet, le principal risque associé à l'étude de sujets sensibles est l'interruption (l'abandon) de la recherche pour des raisons de perturbation sérieuse du processus (dangers physiques, non accès aux données du terrain, contrôle 
externe du processus de recherche). Dans le prolongement des travaux de Lee et Lee (2012), l'analyse réalisée permet d'établir sept grandes catégories de terrains sensibles auxquels peuvent être soumis des chercheurs en sciences de gestion et du management :

- Les terrains dédiés à des zones géographiques à risques, où l'intégrité physique et morale du chercheur est menacée à tout moment pour cause de guerre ou d'instabilité politique ;

- Les terrains d'entreprise à forts enjeux stratégiques, nécessitant des précautions de confidentialité, où la présence du chercheur peut venir perturber le jeu des acteurs et amener les acteurs interrogés à des réactions de stress, d'anxiété ou de rejet (changements structurels, restructuration, réorganisation, fusion interne, intégration post-acquisition, etc.) ;

- Les terrains dédiés à des groupes sociaux ou culturels en marge de la norme sociale ou sociétale, où la simple présence du chercheur peut engendrer des réactions d'hostilités, ou à tout le moins de défiance de part de populations cible (délinquants, terroristes, fanatiques, groupuscules, sans domicile fixe, sans-abri, migrants, clandestins, marginaux, illégaux, etc.) ;

- Les terrains dédiés à des groupes vulnérables en situation de manque ou de carence, où la présence du chercheur peut engendrer des réactions émotionnelles fortes de part du chercheur et des populations cible (personnes seules et démunies, femmes battues, adolescents fugueurs, personnes en état de forte pauvreté, etc.) ;

- Les terrains dédiés à des groupes militants ou politiques, où la présence du chercheur peut engendrer des réactions d'hostilités, ou à tout le moins de défiance de la part de populations cible ayant un engagement militant affirmé (partis politiques, syndicats, associations militantes, mouvements sociaux, mouvements écologiques, etc.) ;

- Les terrains sur des sujets sensibles "tabous " (convictions religieuses, convictions politiques, sexualité, alcoolisme, handicap, etc.), relevant de la vie privée des individus et qui touche à leur identité personnelle ;

- Les terrains portant sur des sujets éthiques ou moraux (racisme, antisémitisme, homophobie, misogynie...), relevant de l'ensemble des pratiques et comportements rejetés collectivement par la Société.

À l'appui de cette typologie qui identifie les différentes formes de risques associées à une recherche en terrain sensible, nous allons chercher à mieux cerner et expliquer les problèmes spécifiques que pose ce type de recherche. Comme nous le verrons, la diversité des situations sensibles en matière de recherche pose des questions morales et éthiques mais également de nature pratique et méthodologique.

\section{Les difficultés ou problèmes rencontrées sur les terrains sensibles en sciences de gestion et du management}

En terrain sensible, le chercheur est exposé à plusieurs types de risques (Sluka, 1990 ; Lee, 1993 ; Agier, 1997 ; Fernandez, 2005 ; Boumaza et Campana, 2007), qui peuvent à tout moment interrompre le processus de recherche et obliger le chercheur à 
abandonner son terrain d'étude. Dans le cas présent, on entend par danger, une source potentielle de dommage, de préjudice ou d'effet nocif à l'égard du protocole de recherche mis en place ou de la personne en charge du programme de recherche (le chercheur). Dans le cas de terrains sensibles, le premier danger est d'ordre physique et porte sur les menaces extérieures (environnement de travail) qui peuvent peser sur le chercheur et ses équipes, en ce qui concerne leur intégrité physique qui peuvent à tout moment être menacée pour cause d'instabilité géopolitique (guerre, révolutions, conflits), politique (instabilité gouvernementale), économique (crise, récessions), sociale (émeutes, grèves, violences urbaines) et environnementale (virus, bactéries, insalubrité, maladies, produits toxiques, etc.) (encadré 1). Le terrain devenu " dangereux " peut devenir un lieu physiquement inaccessible pour le chercheur.

\section{Encadré 1 : Le cas d'une thèse de doctorat sur la question des inégalités dans l'enseignement supérieur au Liban}

En matière de thèses, prenons le cas de la thèse de N. Nimer (2016) sur la construction des inégalités sociales dans le système éducatif au Liban. Comme le souligne la chercheuse, la thèse s'est réalisée dans un contexte difficile en raison de la situation du pays (instabilité politique et sociale, conflit armé avec l'État islamique en 2015 ; affrontements entre l'État islamique et le Front al-Nosra en 2016), mais aussi de l'importance des cultures et origines au sein de ce pays multiculturel, qui peuvent à tout moment créer des zones de tensions ou discordes entre groupes distincts, avec de fortes inégalités. II s'agit en effet d'un contexte où s'articulent les logiques communautaire, socio-économique et spatiale. Les appartenances confessionnelles s'inscrivent dans des territoires spécifiques, où elles sont majoritaires : par exemple, les musulmans chiites dans le sud et la Bekaa, les druzes et les chrétiens maronites dans le Mont Liban, les musulmans sunnites dans les grandes villes et dans le nord. Elles s'y singularisent aussi par leur propre histoire, leur structure sociale et leur type d'organisation politique, religieuse et éducative. En outre, l'histoire des communautés et de leur rapport à l'État explique leur accès plus ou moins précoce ou tardif à la modernité, à travers l'école. Enfin, les communautés confessionnelles ont occupé des positions différentes par rapport au pouvoir politique, tout au long de l'histoire. Cette chercheuse s'est donc intéressée à un sujet passionnant mais complexe, où l'environnement de travail est analysé sous l'angle d'un " système fragmenté et inégalitaire » au sein d'un pays marqué par une instabilité politique (conflits) et sociale. La chercheuse a par conséquent dû en permanence prendre en compte ces différentes contraintes et faire face à certaines " crispations » et " hésitations » de ses interlocuteurs.

Le second danger est l'incapacité du chercheur à rendre sa démarche légitime auprès des acteurs concernés (populations cible), en générant des réactions d’hostilités, ou à tout le moins de défiance, dans le cadre de ses interactions avec autrui (observations participantes, entretiens, interventions). L'une des responsabilités du chercheur concerne le positionnement du chercheur vis-à-vis de l'ensemble des acteurs qui peuvent affecter ou être affectés par l'objet de la recherche. La notion de légitimité fait ici référence à une démarche qui puisse être " acceptée, reconnue et justifiée " (Accardo et Corcuff, 1986). « Acceptée » entend désigner l'idée l'action menée puisse être admise au sein du groupe étudié. "Reconnue " vise à rendre compte du caractère objectif et scientifique de la démarche, en dehors de tout esprit partisan. « Justifiée » entend montrer de quelle façon la recherche proposée est utile socialement et garantit l'image institutionnelle et morale de l'organisation partenaire ou du groupe 
ciblé. En effet, sur des sujets tabous ou controversés (encadré 2), le chercheur peut rapidement faire face à une incompréhension de la part des personnes interrogées, qui vont marquer une forte distance à son encontre. Le chercheur risque alors d'être stigmatisé et peut même être pris à partie (menaces, pression, intimidation).

\section{Encadré 2 : Le cas d'une thèse de doctorat sur la gestion du voile en entreprise}

La thèse en cours de $S$. Chenigle sur le port du voile et sa révélation témoigne des difficultés auxquelles peuvent être confrontées des chercheurs travaillant sur des sujets tabous. Le chercheur doit gérer un environnement social rapidement prêt à porter un regard sceptique voire critique sur le travail proposé. Malgré les précautions prises, le chercheur est confronté à des a priori négatifs puissants qui conduisent à entraver son travail, que cela soit dans la collecte de données comme dans la restitution des analyses. Construire une recherche dans un contexte qui, loin de s'apaiser, est sans cesse soumis à de nouveaux évènements le rendant de plus en plus sensible, place le chercheur dans une situation de tension importante. La situation peut être encore plus difficile lorsque le chercheur tente de faire reconnaitre son travail auprès d'un plus large public. Ici, les résultats sont ambivalents. D'un côté, le chercheur acquiert une reconnaissance, une visibilité institutionnelle lui témoignant de l'intérêt que peut avoir son sujet qui n'a pas toujours été compris comme en témoignent plusieurs refus lors de congrès scientifiques. De l'autre, cela l'oblige à devoir gérer des réactions d'une grande violence de la part de lecteurs outrés de l'existence même de ce type de travail. C'est ici que le directeur de thèse comme soutien est essentiel. Tout en aidant la doctorante à garder sa distance par rapport à la population interrogée, en lui rappelant l'exigence de distanciation, le directeur de thèse doit entretenir la motivation d'une personne dans le doute ne pouvant compter ni sur une reconnaissance académique, ni sur celle d'une société civile craintive, voire rétive. On peut aussi souligner l'importance de la structure de laboratoire dans laquelle le doctorant peut retrouver une convivialité le rassurant sur sa valeur comme sur la possibilité de réussir sa recherche malgré les difficultés.

Ce type de protocole est bien illustré par le retour d'expérience de Richaud (2016) qui rapporte ses questionnements et réponses méthodologiques en réaction à la pression exercée par le système de contrôle de l'État, alors qu'elle menait une enquête sur les rassemblements de retraités dans un parc public de Pékin. Dans ce domaine, la présentation de soi et la fourniture de garanties de neutralité ne suffisent pas. Cette chercheuse s'est trouvée en permanence confrontée au défi pragmatique de mener une recherche sous la surveillance suspicieuse et pesante d'agents de la sécurité chinoise, engendrant un climat paranoïaque qui l'a obligée à mettre en œuvre des mesures d'autoprotection pour éviter toute accusation d'espionnage. La chercheuse s'est d'abord sentie obligée de fournir des "gages " afin de prouver sa neutralité, en " surjouant " les stéréotypes comportementaux du chercheur, seul moyen de réaffirmer sa présence face à la suspicion. La manière de se comporter et le choix de la mise en scène sont ainsi devenues aussi cruciales que la conduite de la recherche. Pour mener à bien son étude, Richaud (2016) a dû en permanence composer avec le milieu environnant pour espérer collecter des données intéressantes.

Le troisième type de risque est d'ordre méthodologique et porte sur l'incapacité du chercheur à concilier distance et proximité. En effet, ce type de terrain fait souvent ressurgir des émotions, des peurs, des questionnements (encadré 3). II tend à exposer l'intimité d'autrui et peut susciter des sentiments de rejet face des situations de violence ou d'injustice. Or, dans ce type de situations, le chercheur doit 
gérer seul toutes les formes d'incertitude et d'adversité. Il doit chercher à gérer la tension constante entre l'engagement nécessaire à la compréhension de la réalité étudiée et la distanciation que requiert la méthodologie de recherche. Le chercheur est ainsi confronté à des réalités changeantes et à des formes de contradictions. II devient, malgré lui, un acteur de cette réalité sociale (Wax, 1986). Le chercheur doit ainsi se plier à un rite initiatique, souvent imposé par l'environnement. II s'agit à la fois de valider ses intentions, de prouver sa neutralité mais aussi de faire montre de sa capacité à investir le terrain. Ainsi, à l'instar des travaux d'Ayimpam (2015), il convient d'éviter de prendre systématiquement parti pour les victimes ou une cause considérée comme " juste ". Le chercheur doit s'attacher à cerner aussi le point de vue de l'autre partie (accusateurs, agresseurs, etc.), afin d'en comprendre les ressorts et les mécanismes. Ce travail délicat est ce qui doit permettre de distinguer le militant, le politique, l'écrivain et le journaliste du chercheur.

\section{Encadré 3 : Le cas d'une thèse de doctorat sur les prêtres mariés}

Le travail de recherche entrepris sur le terrain des prêtres mariés constitue un autre exemple de terrain sensible où l'acceptation de la thématique de recherche (la gestion de la transition professionnelle de prêtre à laïc) s'avère délicat. Même si le poids de la religion catholique a diminué dans la société française, réaliser un tel travail, c'est se confronter d'abord à une résistance aigue de la part tant de l'institution que de ces anciens prêtres. Là encore, rentrer en contact avec la population est difficile, le soupçon très présent. Le chercheur est vu comme celui venant donner une image négative de cette transition. II doit faire ses preuves. Pour cela, il doit participer à des réunions organisées par cette population, veiller à garantir anonymat et confidentialité des propos échangés. Plus encore, il convient d'identifier dans la population cible les leaders d'opinion susceptibles d'ouvrir des portes et de cautionner la neutralité du chercheur. Le chercheur est dépendant de l'impression qu'il fait auprès des personnes qu'il interroge en premier. C'est pourquoi une dynamique spécifique d'entretiens doit se mettre en place. Les premières rencontres n'ont pas nécessairement pour but de recueillir immédiatement les informations essentielles pour le sujet de recherche mais visent à se faire accepter pour avoir accès à une population plus large. Il convient donc, dans ce contexte, de penser la collecte de données en deux temps. Dans un second temps, le chercheur, une fois atteint un nombre suffisant d'entretiens, proche de la saturation sémantique, doit revenir vers ses premiers interlocuteurs pour approfondir les thématiques potentiellement conflictuelles qu'il n'a fait qu'aborder sans les approfondir. Ce double mouvement dans la collecte apparaît comme une spécificité des terrains sensibles, tant une mauvaise réputation du chercheur se construit rapidement et devient synonyme de fermeture du terrain.

Dickson-Swift et al. (2006) soulignent, à partir du cas des recherches dans le domaine de la santé, que la gestion de la proximité avec les personnes interrogées constitue un défi particulièrement délicat, tant ses formes sont diverses. Premièrement, le chercheur peut s'interroger sur la nature réelle de son travail qui peut s'assimiler au développement d'une thérapie ou d'entretiens d'orientation visant en premier lieu non pas à créer de la connaissance, mais à aider des personnes en situation difficile. Deuxièmement, le chercheur peut développer des relations d'amitié en voulant créer de la proximité avec les personnes interrogées. Lee (1993) souligne cette difficulté en suggérant l'existence d'une porosité qui interroge entre amitié et distance pour la construction d'un raisonnement scientifique. La difficulté de cette mise à distance empathique conduit, selon Dickson-Swift et al. (2006), à prendre le risque d'une 
gestion émotionnelle avec les participants à son terrain de recherche. Cette nouvelle dimension peut conduire alors à avoir des difficultés lors de la fin de la recherche qui peut être associée à une situation de burnout. Cette dimension émotionnelle de la recherche sur des terrains sensibles rappelle la difficulté de la distance scientifique à l'égard du terrain d'enquête qui doit être pensée non pas seulement pendant le travail de recherche, mais aussi après.

Un autre risque important est la question de la récupération et l'instrumentalisation des résultats de la recherche par le milieu socioculturel local et l'ensemble des personnes concernées par l'étude (encadré 4). En effet, s'intéresser à des questions extrêmes ou tabous, relatives par exemple à des engagements politiques ou religieux dans la sphère publique peut être perçu par certains acteurs impliqués dans le processus (chercheur, directeur de recherche, personnes interrogées) comme un moyen de défendre ou de combattre une cause militante sous couvert d'une démarche d'orientation scientifique. Au-delà de l'honnêteté intellectuelle du chercheur, il importe donc à celui-ci de fournir des gages sur sa capacité à traduire et analyser scientifiquement des données de façon rigoureuse et non partisane.

\section{Encadré 4 : Le cas des thèses de doctorat sur le syndicalisme}

Le terrain réalisé autour de la thématique de la trajectoire professionnelle des leaders syndicaux après leur investissement dans une lutte contre la fermeture de leur entreprise constitue un exemple des difficultés liées à la récupération, voire la polémique pouvant entourer une recherche sur un terrain sensible. En effet, cette recherche interroge à la fois les structures syndicales sur leur capacité à accompagner des militants engagés ayant accepté de mettre leur employabilité en jeu pour le bien du collectif et les employeurs quant à l'existence de listes noires de syndicalistes ne pouvons retrouver d'emplois dans leur région du fait de leur investissement dans un conflit. Plus encore, nos données montrent que certains syndicalistes ont accepté de bénéficier de pratiques éthiquement questionnables pour favoriser leur rebond professionnel. On le voit, cette recherche regroupe des possibilités de récupération de multiples acteurs ayant tous contribué à la réalisation du travail. Dans ce contexte, pour éviter une récupération des résultats que chacun peut biaiser en sa faveur, le chercheur doit veiller à toujours communiquer sur l'ensemble des dimensions relevées. C'est pourquoi les résultats du travail furent communiqués de manière officielle au travers de propositions d'articles à des revues académiques de telle sorte qu'aucun des acteurs ne puisse se saisir des résultats. De la même manière, les retours aux interviewés furent réalisés sous une forme académique. En maitrisant soigneusement l'accès aux données, le chercheur peut s'assurer un contrôle de l'instrumentalisation de ses travaux. Cependant, cette stratégie d'isolement scientifique ne favorise pas les mises en relation futures avec le terrain étudié tant ce dernier est en attente de communication accessible. Ceci peut conduire le chercheur à vouloir donner accès à son travail de manière plus lisible à ses interviewés. Cette stratégie, que nous n'avons pas retenue dans cette recherche, apparaît pour nous plus délicate tant elle peut amener le chercheur à devenir l'objet de polémiques.

Lorsqu'on mène des recherches sur des terrains sensibles, il y a également un risque quant à la confidentialité des résultats qui peut fortement limiter la portée et l'ampleur de la contribution finale. En effet, dans des terrains sensibles de nature stratégique (fusions-acquisitions, restructurations, etc.), politique (actions gouvernementales) ou religieuse (pratiques religieuses), il est d'usage que les instances concernées (directions, comité de direction, etc.) puissent avoir un regard critique 
sur la production de la recherche menée et demandent une révision ou l'interdiction de certaines contributions jugées néfastes pour leur institution ou leur organisation. Or, bien souvent, l'intérêt d'une recherche est de révéler ou dévoiler au sens de Bourdieu (2007), des situations et relations inédites qui peuvent faire progresser la science, en remettant en cause ou en améliorant l'étude de certains phénomènes. La conscience du chercheur est donc mise à mal entre sa volonté de respecter le " contrat psychologique " qu'il a signé avec l'institution concernée et sa quête de publier des résultats spécifiques qui puissent améliorer le champ de la connaissance.

\section{Encadré 5 : Le cas des thèses sur le management de fusions-acquisitions}

En matière de thèses de doctorat, prenons le cas particulier des fusions-acquisitions qui illustrent l'extrême sensibilité à la diffusion des données collectées et à la confidentialité des résultats. Au-delà de caractéristiques communes à l'ensemble des décisions stratégiques, les auteurs s'accordent à dire que le risque pour le dirigeant sous pression de prendre des décisions erronées (mauvais choix de la cible, par exemple) est plus élevé lors de fusions-acquisitions, compte-tenu du contexte d'urgence qui entoure ce type d'opération, de son degré élevé de visibilité et du caractère fortement asymétrique de la relation (Haspeslagh et Jemison, 1991). Le champ des fusions-acquisitions constitue par conséquent un sujet sensible à traiter, en raison à la fois de sa dimension stratégique et politique, qui va obliger le chercheur à négocier et garantir son accès au terrain (Duforez, 1995 ; Meier, 1998). En effet, la qualité des investigations est fortement dépendante du maintien de la présence du chercheur sur le terrain et de la possibilité d'exploiter les opportunités en termes de collecte des données (enquête, entretiens, observation participante). Comme le souligne Huchette (2020), dont la thèse de DBA porte sur un processus d'intégration organisationnelle post-fusion en milieu universitaire, c'est sans discontinuer qu'il convient de rester discret quant à la recherche, pour réussir à se faire oublier et éviter de relancer les négociations indéfiniment. Cela nécessite de la part du chercheur des qualités de persuasion, de négociation et de diplomatie pour expliquer la démarche et faire comprendre le besoin de collecter des informations. Mais à n'en pas douter, l'une des difficultés majeures demeure la capacité à pouvoir publier les résultats de la recherche sans pour autant nuire à l'image de l'organisation et à son positionnement dans l'environnement concurrentiel. C'est pourquoi il importe dans ce type de terrain d'obtenir officiellement l'autorisation de la direction du nouvel ensemble et d'informer précisément les participants de l'objectif de la recherche, ceci pour éviter les risques d'incompréhensions (réticences, résistances, méfiance) et de propagation de rumeurs (suspicions). En effet, l'un des problèmes récurrents de ce type de recherche est de se voir, après plusieurs mois d'enquête, refuser l'accès aux données pour cause de changements stratégiques (changement de stratégie ou diffusion de certaines pratiques à la concurrence), politiques (conflits d'intérêt) ou sociaux (hostilité, tensions, conflits).

\section{Solutions et recommandations}

Dans le cas de la gestion de terrains sensibles se pose inévitablement la question la responsabilité du chercheur auprès des populations étudiées, mais aussi des personnes concernées par le sujet d'étude. En particulier, le chercheur se doit de répondre à trois principales difficultés que sont la définition du positionnement de la recherche (engagement vs. neutralité), la gestion du terrain (investigation vs. sécurité) et sa capacité à restituer des résultats pertinents dans des publications et auprès des organisations concernées (officialisation de la recherche vs. confidentialité). 
L'étude de terrains sensibles présente comme difficulté préalable l'accès au terrain. Elle nécessite par conséquent le recours à des stratégies mixtes de collecte des données afin, d'une part, d'éviter une trop grande polarisation des sources d'informations sur des profils extrêmes (populations cibles) pouvant fortement orienter le sujet et, d'autre part, assurer une continuité de l'accès aux données en dépit de circonstances défavorables (environnement menaçant, danger physique, rétractation de certains acteurs, hostilités de la part de certains acteurs). C'est pourquoi il est important que le chercheur puisse recourir à différentes sources d'informations (populations cible, instances officielles et officieuses, citoyens, consommateurs, utilisateurs, observateurs, interactions avec d'autres chercheurs ou partenaires extérieurs au projet, journaux, blogs et forums de discussion sur Internet, etc.). Par ailleurs, il importe de mobiliser plusieurs méthodes de collecte : observations participantes, entretiens semi-directifs, réunions collectives, récités de vie, relations formelles et informelles, etc.). Plus encore, Beji-Bécheur et al. (2011) soulignent l'importance d'intégrer dans une phase amont de la relation au terrain une intense introspection quant à sa compréhension du sujet et la relation personnelle de chaque chercheur à la thématique de recherche. Le chercheur est invité à réfléchir sur sa relation personnelle avec le sujet. Articulant phase narrative et métacognitive, avant d'approfondir sa compréhension théorique du sujet, il débat avec les autres membres de l'équipe de la thématique de recherche à partir de son histoire personnelle.

En matière de comportement, face aux risques de surexposition à des situations émotionnellement fortes, déstabilisantes ou impliquantes, le chercheur se doit de définir ses propres repères, en veillant à ne pas se laisser submerger par ses émotions ou à sortir de façon trop marquée de son rôle de chercheur. C'est pourquoi le travail sur soi, visant à reposer les bases de son métier, de son positionnement dans la société et de ses responsabilités, peut constituer une base de protection nécessaire à l'équilibre psychique du chercheur et la qualité de son travail. L'identité professionnelle du chercheur pour gérer la tension proximité / distance et pour garder une cohérence d'ensemble est une solution intéressante. Plusieurs chercheurs féministes, dont Acker et al. (1983), soulignent que cette volonté de maintenir une frontière stricte entre distanciation scientifique et proximité amicale demeure sur le principe raisonnable mais, dans les faits, beaucoup plus difficile à tenir. Somers et al. (1982) rappellent que le succès d'une recherche suppose l'articulation de deux principes. Le premier est le respect des droits des interviewés notamment en termes de respect de leur vie privée et de la confidentialité des données collectées. Le second est d'intégrer qu'une recherche académique doit articuler les intérêts des interviewés comme de tout ceux étant dans une situation comparable. Sans questionner ces deux principes directeurs, il convient de souligner que leur application stricte peut conduire à une sanctification des interviewés qui pose d'autres questions, notamment lorsque la recherche s'interroge sur des comportements déviants des participants.

Au cours de son investigation, le chercheur va devoir se construire un rôle qui combine neutralité et complicité, en s'inscrivant dans un rôle qui soit en accord avec ce qu'attendent les interviewés (Marcus, 1997). Ces relations avec la population considérée, sont une façon de minimiser les dangers et les risques (Kovats-Bernat, 2002). II peut aussi être parrainé par des intermédiaires (ou gatekeepers) qui vont faciliter le contact, par exemple des associations, des organisations gouvernementales ou des syndicats. Le chercheur est ainsi confronté à des réalités changeantes et à des 
formes de contradictions. Il devient, malgré lui, un acteur de cette réalité sociale (Wax, 1986). Le chercheur doit ainsi se plier à un rite initiatique, souvent imposé par l'environnement. II s'agit à la fois de valider ses intentions, de prouver sa neutralité, mais aussi de faire montre de sa capacité à investir le terrain. Des premiers contacts dépendra la nature de sa relation avec le terrain d'étude. Le poids de ces exigences, particulièrement fort dans le cadre des travaux médicaux, a conduit de nombreux pays à étendre le système de supervision éthique traditionnellement réservé au champ de la biomédecine. Herdman (2000) souligne toutefois que les exigences de ce type de structures conduit à un appauvrissement de la créativité et questionne la liberté d'investigation du chercheur.

Loin d'être une cause entendue, la neutralité comme la bienveillance du chercheur en sciences sociales est un sujet où les débats restent ouverts. Ger et Sandikci (2006) soulignent que la perception des principes édictés par Somers et al. (1982) est marquée par la subjectivité. C'est pourquoi, dans le prolongement de Sieber (1993), il convient que le chercheur soit avant tout sensible culturellement plutôt que normativement, ce qui pourrait le conduire soit à ne pas pouvoir mener sa recherche, soit à rencontrer des difficultés dans sa diffusion. Adopter un tel ancrage conduit à repositionner la question de la prise en compte de la sensibilité d'un sujet de recherche à un niveau dépassant celui des supervisions éthiques propres à une culture anglosaxonne. Il apparaît que la prise en compte des intérêts et des droits des interviewés suppose un chercheur intégrant dans sa réflexion les normes sociales qui s'appliquent dans le contexte de sa recherche. Ger et Sandikci (2006) soulignent que l'intégration stricte de cette prise en compte peut nuire à la production de connaissance tant il peut supposer des demandes d'autorisation, par exemple pour prendre des photographies ou réaliser certaines observations participantes. De telles contraintes conduisent ainsi à influencer le comportement des personnes observées. On constate donc ici l'existence d'une zone délicate de la pratique de la recherche en terrain sensible. Ainsi, faire de la recherche en sciences sociales sur des terrains sensibles, c'est être en permanence à la marge de son champ disciplinaire, avec sans cesse la possibilité de se faire critiquer, voire exclure tant de son terrain (partie prenante) que de sa communauté académique.

Le positionnement du directeur de thèse face à la recherche sensible de son doctorant est lui aussi complexe. Nous fondant sur notre expérience, plusieurs points apparaissent comme essentiels. Le premier est de faire prendre conscience au doctorant du caractère sensible de son terrain. En effet, sa familiarité avec la thématique peut lui faire perdre cette distanciation. Le directeur de thèse joue ici le rôle de celui qui met à distance, rappelle que le sujet peut être compris avec des a priori que le doctorant trop pris par son engagement oublie. II rappelle au doctorant que le travail de thèse n'est pas un acte militant mais doit avoir une dimension critique que seule la distanciation peut permettre. En cela, le directeur de thèse doit avoir conscience que sa seule influence ne suffira pas à canaliser la proximité du doctorant, qui peut rimer avec un engagement quasi-militant. Pour éviter à ce dernier une confrontation difficile tant avec la communauté scientifique que civile, il doit pouvoir compter sur l'appui du comité de suivi de thèse, d'autres chercheurs dans son laboratoire, voire sur ceux rencontrés lors de congrès ou de journées de recherche.

Le deuxième point est d'accompagner le doctorant lors des moments difficiles où le lien au terrain peut être questionné par la population interrogée comme par 
l'institution universitaire qui peut s'interroger sur l'intérêt de la thématique. Là, s'appuyant sur son positionnement institutionnel, il peut aider le doctorant en le protégeant par un écrit ou par sa présence lors d'entretiens garantissant la qualité scientifique de sa démarche le distinguant d'un travail journalistique ou de consultance. Il peut aussi l'accompagner dans la rédaction de ses propositions d'articles ou de communications. En initiant une dynamique de publication avec le doctorant, il lui montre l'intérêt que possède son sujet malgré les refus auxquels il doit faire face. Enfin, troisième point, le directeur de thèse doit veiller à identifier dans l'environnement les personnes susceptibles d'apprécier son travail. On retrouve là une tâche classique du directeur de thèse mais son importance est décuplée par la possible résistance au sein de la communauté de chercheurs peu ouverts à cette thématique.

Ceci nous ouvre à une dernière dimension, qui à nos yeux demeure centrale mais n'est pas abordée, à notre connaissance par la littérature : la prise de risque pour le directeur de thèse. En effet, en soutenant des doctorants s'orientant vers des terrains sensibles, le directeur de thèse s'expose, s'identifie comme une personne pouvant avoir des convictions susceptibles d'être minoritaires dans la société, voire la communauté scientifique. C'est pourquoi avant d'entamer une telle démarche, il convient d'être au clair sur ces dimensions pour pouvoir les assumer et ne pas prendre des risques éthiques forts tels que laisser seul le doctorant face aux conséquences sociétales et académiques d'un investissement sur un terrain sensible. Sur ces bases, il apparaît que la direction d'un doctorant investi dans un terrain sensible suppose de la part du directeur de thèse une prise de risque que son expérience doit lui permettre de mieux maitriser que le doctorant qu'il encadre. Néanmoins, la question demeure posée de savoir s'il ne conviendrait pas de veiller aussi sur le directeur de thèse pour l'aider à trouver la bonne distance avec son doctorant. Pour notre cas personnel, nous avons développé des ateliers avec d'autres chercheurs travaillant sur ces sujets afin de constituer une communauté de recherche apte à partager le vécu à la fois du directeur de thèse et de son doctorant (encadré 6).

\section{Encadré 6 : Retour d'expériences de thèses de doctorat en terrains sensibles}

Notre expérience de directeurs de thèses dans le domaine nous amène à formuler plusieurs remarques. Lorsque nous sommes confrontés à des terrains à risques, notre principale crainte en tant que chercheur est le risque de rupture du projet pouvant émaner des populations concernées (désapprobation, désaccord, sanction) ou de nous-même (impossibilité pour le doctorant de poursuivre la recherche pour cause d'obstructions). En effet, généralement, ce type de terrains a été difficile à trouver et à obtenir au niveau de son accès et de la collecte des données. Il a souvent demandé du temps, des investigations préalables (observations, entretiens) et de la négociation. Dès lors, le directeur de recherche se trouve très vite confronté à une responsabilité qui l'obsède, à savoir comment assurer une recherche de qualité à son doctorant dans un contexte qui peut à tout moment " déraper " en raison de son caractère sensible. Le directeur de thèse est donc, malgré lui, dans une position inconfortable qui va l'amener dans un premier temps à veiller coûte que coûte à assurer la viabilité du terrain, afin de protéger son doctorant pour que ce dernier puisse mener à bien sa thèse. II y a donc au départ une démarche opportuniste et pragmatique que l'on ne peut nier, liée à la satisfaction d'avoir eu accès à ce type de recherche.

C'est au cours du processus que la vraie difficulté commence, lorsque le doctorant commence à collecter des données intéressantes et que les premières analyses font apparaître des résultats prometteurs. C'est à ce moment-là que le directeur de thèse se trouve en proie à différentes injonctions paradoxales, devant commencer à aider à la 
valorisation de la recherche (analyse critique et rigoureuse, objectivation des résultats), tout en veillant à ce qu'aucune décision ou événement ne remette en cause le travail produit. Cette difficulté est d'autant plus grande lorsque le terrain dépend d'autorités supérieures sur lesquelles la recherche s'est appuyée pour collecter les données. II faut donc à la fois rester en phase avec le terrain et sa culture propre au risque d'être marginalisé ou sanctionné, tout en s'affranchissant de pressions sociales, culturelles ou politiques pouvant nuire à la qualité de la thèse. Le véritable défi d'un directeur de thèse est donc ici avec son thésard d'être capable de proposer des résultats conformes à la réalité socio-culturelle du milieu dans lequel ils évoluent, tout en gardant une capacité critique et autonome en termes d'analyse et d'interprétation. Un chercheur en terrain sensible ne doit donc pas simplement montrer des qualités d'observation et de réflexion; il doit aussi faire preuve de qualités pédagogiques et diplomatiques pour faire accepter des résultats qui ne sont pas toujours en phase avec les orientations initiales des instances concernées, sans pour autant se faire accuser d'esprit partisan. Rappeler en permanence les objectifs de la thèse, faire preuve de pédagogie, montrer le sérieux du protocole, rester ouvert aux remarques de l'extérieur sont autant d'actions pouvant contribuer à atteindre cet objectif.

Un autre aspect du sujet concerne la relation complexe entre le terrain sensible et l'objet tabou, comme le révèlent les travaux de Peretti-Ndiaye (2016) sur la question épineuse du racisme et notamment de l'expérience relationnelle à double face, où le visible côtoie l'invisible (gêne, méfiance, comportements fuyants, politiquement correct, etc.). Lors de son enquête en Corse, elle a dû élaborer des stratégies d'observation clandestines et des processus d'évitement dans la présentation de son objet de recherche. Ces stratégies ont eu pour effet de la confronter à un autre versant du problème : l'expérience de situations limites. Elles interrogent sur le rôle et la place du chercheur dans l'univers social et sur la nature des liens que celui-ci entretient avec les personnes interrogées. Où placer le curseur entre les considérations éthiques et la rigueur méthodologique, quand on est amené à observer à leur insu certaines populations, interroger des victimes qui refusent de parler, d'entrer en communication avec des acteurs qui ne nous laissent pas indifférents, en termes d'empathie ou antipathie (absence de neutralité) ? Où se positionner?

Au-delà de l'objet tabou, l'accès au terrain peut être rendu périlleux, en raison de l'absence d'autorisations institutionnelles (guerre, conflits, violences urbaines, etc.), le terrain devenu " dangereux " peut devenir un lieu physiquement inaccessible pour le chercheur. Le chercheur investi doit alors trouver des solutions alternatives. Aujourd'hui, les nouvelles technologies de communication permettent ainsi d'échanger des informations avec des acteurs restés sur le terrain. Cette " solution " de contournement de l'impossibilité de se déplacer physiquement pose néanmoins la question de la distance, celle qui nous sépare du milieu que l'on cherche à comprendre.

\section{Conclusion}

Les recherches qui s'intéressent à la gestion des terrains sensibles sont encore peu développées, en dépit de changements sociaux, sociétaux, culturels et technologiques, dont l'ampleur vient envahir la sphère publique et privée et, plus globalement, le monde de l'entreprise. Or, ces changements viennent fortement remettre en cause un certain nombre de pratiques et de comportements rationnels légaux 
sur lesquels se sont construites nos sociétés (valorisation de la science, importance des outils cognitifs, primauté accordée à l'économie, recherche de stabilité interne, domination de la nature). Ce type de recherches est donc essentiel pour mieux comprendre les transformations à l'œuvre dans nos sociétés et apprendre à gérer concrètement et durablement la complexité et la diversité.

Faire le choix de mener une recherche à partir d'un terrain sensible, c'est donc accepter de confronter la population interrogée à de multiple risques (intégrité physique, pressions politiques, pressions sociales et culturelles, intimidations), mais aussi se confronter à des risques tout aussi importants en tant que chercheur (absence de financement, entrave à son indépendance, abandon de ses terrains d'études, menace physique ou institutionnelle). La littérature souligne l'importance des deux dimensions sans pour autant proposer de solutions pour y faire face autres que dissuader de potentiels chercheurs de s'intéresser à ses thématiques sensibles, tant la fragilité des populations interrogées sera source de difficultés méthodologiques et éthiques.

Ce chapitre se veut un premier pas vers un rééquilibrage face à un mouvement que nous percevons comme préoccupant, qui consiste à construire des obstacles à la création de connaissances pour protéger des populations fragiles. Sans nier une telle fragilité, notre position est de rappeler qu'elle ne doit pas être assimilée à l'absence de capacité d'agence. Le chercheur, qu'il soit doctorant ou pas, son directeur de thèse, voire son laboratoire, sont eux aussi impliqués dans une recherche potentiellement source de tensions académiques et sociales. Pour faire face à ces difficultés, le chercheur ne doit pas seulement avoir des bases en sciences de gestion et du management mais aussi prendre conscience que son travail suppose la création d'une base de légitimité auprès de différentes parties prenantes tant dans la sphère académique que dans la société au sens large. Sans aller jusqu'à mener des démarches d'ethnographie critique (Thomas, 1993), il convient de garder à l'esprit que la recherche sur un terrain sensible constitue un défi professionnel qui témoigne de la volonté du chercheur de créer une connaissance suscitant l'intérêt et le débat dans le champ des sciences de gestion et du management. En effet, nous pensons que la production de connaissances sur la gestion des terrains sensibles est fortement liée avec l'objet même de ces sciences, à savoir aider les hommes et les femmes d'action à agir, pour mieux comprendre et faire face à l'adversité. 


\section{La logistique \\ Un champ disciplinaire singulier des sciences de gestion et du management}

François Fulconis et Gilles Paché

La crise du coronavirus au printemps 2020 a souligné le caractère central des problématiques logistiques, qu'il s'agisse de la paralysie des chaînes globales de valeur, de l'approvisionnement défaillant des masques de protection et de la gestion critique des capacités d'accueil des malades en détresse respiratoire. Même lorsque l'heure du déconfinement est venue, la logistique a été à nouveau évoquée à maintes reprises comme clé de la relance de la « machine économique ». Doit-on s'étonner d'un tel engouement ? La réponse est positive et négative à la fois. Positive car l'intérêt des plus hautes sphères du pouvoir politique pendant la pandémie de Covid-19 détonne avec leur relatif dédain, pendant des décennies, pour des activités de transport relevant d'une simple dimension infrastructurelle. Négative car de nombreux chercheurs et praticiens en dehors du champ de la logistique lui reconnaissent depuis longtemps une importance centrale dans le développement d'un avantage concurrentiel durable.

Force est pourtant de reconnaître que, sur un plan académique, notamment au sein des instances d'évaluation, qu'elles soient internationales, nationales ou locales, la logistique connaît parfois (souvent ?) un déni de légitimité dans son versant managérial. Dit autrement, pour de nombreux évaluateurs, la logistique " scientifique " serait avant tout de nature ingénierique, fondée sur des logiques d'optimisation sous contrainte, et relevant d'une rationalité substantive, et certainement pas procédurale. L'ouvrage de Simchi-Levi et al. (2014) en constitue une remarquable illustration. L'une des manifestations les plus éclatantes du triomphe de la rationalité substantive reste certainement le classement des revues considérées comme faisant référence en matière de recherche en logistique, et le type d'articles qu'elles y publient majoritairement. On pourrait également signaler le profil des dossiers des candidats promus aux fonctions de professeur des universités en sciences de gestion et du management, dont un nombre non négligeable possède un background dominant en sciences de l'ingénieur. 
II n'est évidemment pas question de porter un jugement critique sur des dossiers de qualité, voire de grande qualité, mais de s'interroger sur la sous-estimation délétère du versant purement managérial de la logistique, dont les processus décisionnels n'échappent pas, dans les faits, à la problématique de la rationalité limitée de Simon (1997), mais aussi aux logiques béhavioristes que l'on retrouve dans la théorie des canaux de distribution, ou encore aux modèles de prédation et de régimes de pouvoir développés notamment par Cox et al. (2001). En bref, comprendre la dynamique des chaînes logistiques, c'est mobiliser un appareillage conceptuel qui n'ignore pas les jeux d'acteurs tels qu'ils structurent, déstructurent et restructurent les organisations et les relations inter-organisationnelles. De ce point de vue, une vision décloisonnée qui associe la logistique à d'autres champs disciplinaires des sciences de gestion et du management apparaît indispensable pour sortir de l'impasse dans laquelle une vision purement technicienne pourrait la conduire, en privilégiant maladroitement l'agent plutôt que l'acteur.

\section{Prendre en compte des logiques d'acteur}

Il y a une vingtaine d'années, Lebraty (2000), interrogé sur la question de qu'est-ce que "faire de la recherche en logistique? ", interpellait les chercheurs en sciences de gestion et du management sur le fait que la logistique semblait profondément liée à une approche optimisatrice de l'entreprise, trouvant ainsi ses limites au niveau managérial. II dressait alors le constat suivant :

\footnotetext{
Causes, enjeux et limites du développement logistique font de ce domaine une discipline complexe, en situation non stabilisée et dont l'évolution dépend, dans le futur, de l'aptitude des universitaires, des praticiens et des consultants à relever un certain nombre de défis que nous situerons, pour schématiser, dans trois domaines : scientifiques, managérial et pédagogique.
}

Depuis, ce champ disciplinaire s'est largement affirmé et la communauté scientifique s'est structurée, notamment dans l'espace francophone autour de l'Association internationale de recherche en logistique et supply chain management (AIRL-SCM), en tentant de relever ces trois défis donnant à la logistique une véritable dimension managériale et organisationnelle.

\section{Rationalité substantive, rationalité procédurale}

Depuis les années 1990, la communauté scientifique a peu à peu pris conscience que la logistique ne se limite ni à des questions d'optimisation, ni aux frontières de l'entreprise, mais qu'elle concerne la politique générale de toute entreprise, qu'elle place le pilotage des interfaces intra- comme inter-organisationnelles au cœur de leur compétitivité et qu'elle requiert une approche transversale des organisations. Tixier et al. (1996) affirment que la logistique d'entreprise n'est plus une " affaire de techniciens ", mais un concept dont la maîtrise constitue " un enjeu stratégique de grande ampleur (...) une arme déterminante pour la compétitivité des entreprises ». Toutefois, à ce moment-là, une difficulté demeure : I'absence de définition synthétique de la logistique. Peut-être est-ce lié au fait que ce terme prend alors de plus en plus d'ampleur dans la vie des entreprises et, de ce fait, dans les recherches 
académiques ? En résumé, si l'importance de la logistique semble reconnue tant au plan professionnel qu'académique, elle est considérée pour certains comme une simple opération élémentaire (gestion des stocks, gestion de production, distribution physique, transport, etc.), alors que d'autres lui attribuent un rôle stratégique de premier ordre (Aurifeille et al., 1997).

Par la suite, des travaux privilégiant une conception englobante de la notion de logistique, dans une perspective à la fois dynamique (en termes de potentiels) et transversale (entre fonctions d'une entreprise et entre entreprises impliquées dans le fonctionnement d'une même chaîne de valeur), sont venus combler ce manque. À partir des réflexions menées principalement dans le champ du management stratégique et des théories des organisations (Chandler, 1962 ; Ansoff, 1965 ; Drucker, 1967 ; Percerou, 1984 ; Martinet, 1993 ; Koenig, 1996), la notion de logistique est enrichie par la confrontation aux potentiels de compétitivité définis tant en termes de capacités opérationnelles (logique d'exploitation) que de capacités stratégiques (logique d'exploration) (Fulconis, 1999). En termes opérationnels, la conception traditionnelle de la logistique, la limitant à un ensemble de méthodes et de moyens relatifs à l'organisation d'un service ou d'une entreprise, est ainsi élargie par une conception privilégiant une approche processuelle et transversale nécessitant la maîtrise des flux logistiques internes et externes à l'entreprise. En termes stratégiques, la logistique est admise comme un processus pour s'adapter, voire pour s'imposer, constituant dans la durée un réel levier de compétitivité pour les entreprises. Elle devient un support à la mise en œuvre de stratégie, voire une stratégie à part entière. Ces réflexions sur l'essence même de la notion de logistique conduisent à une conceptualisation de la notion de « management logistique » que synthétise le tableau 1.

Tableau 1 : Le concept de «management logistique » : décomposition et articulation des logiques d'exploitation et d'exploration

\begin{tabular}{|c|c|c|c|c|}
\hline CONCEPT & \multicolumn{4}{|c|}{ MANAGEMENT LOGISTIQUE } \\
\hline Dimensions & \multicolumn{2}{|c|}{ Opérationnelle } & \multicolumn{2}{|c|}{ Stratégique } \\
\hline Logiques & \multicolumn{2}{|c|}{ Exploitation } & \multicolumn{2}{|c|}{ Exploration } \\
\hline $\begin{array}{l}\text { Acceptions } \\
\text { de la logistique } \\
\text { (composantes) }\end{array}$ & Activité de support & $\begin{array}{c}\text { Technologie } \\
\text { de la maîtrise des flux }\end{array}$ & $\begin{array}{l}\text { Support à la mise en } \\
\text { œuvre de la stratégie }\end{array}$ & $\begin{array}{l}\text { Stratégie } \\
\text { à part entière }\end{array}$ \\
\hline $\begin{array}{c}\text { Caractéristiques } \\
\text { (indicateurs, attributs) }\end{array}$ & $\begin{array}{l}\text { Ensemble } \\
\text { de méthodes et } \\
\text { de moyens relatifs à la } \\
\text { gestion des stocks, } \\
\text { à l'organisation } \\
\text { d'une fonction } \\
\text { ou d'une entreprise }\end{array}$ & $\begin{array}{l}\text { Flux expédiés } \\
\text { vers le client, transférés } \\
\text { entre ou au sein } \\
\text { de fonctions de } \\
\text { l'entreprise et reçus } \\
\text { des fournisseurs }\end{array}$ & $\begin{array}{l}\text { Moyen par lequel } \\
\text { l'entreprise force sa } \\
\text { capacité d'adaptation } \\
\text { (réaction, anticipation) }\end{array}$ & $\begin{array}{c}\text { Manœuvre } \\
\text { visant à modifier } \\
\text { l'équilibre concurrentiel } \\
\text { selon une approche } \\
\text { pro-active }\end{array}$ \\
\hline
\end{tabular}

C Construction du concept de " management logistique » en fonction des logiques d'exploitation et d'exploration

$\longrightarrow$ Construction du concept de « management logistique » privilégiant l'articulation des logiques d'exploitation et d'exploration

Source : Fulconis (1999)

La conceptualisation de la notion de « management logistique » rejoint la définition proposée ensuite par Colin (2005). Amendant celle avancée par le Council of Supply 
Chain Management Professionals (CSCMP), il affirme que " le management logistique est cette fraction du supply chain management qui prévoit, met en place et maîtrise de façon efficiente et efficace les flux aller et retour de marchandises, leur entreposage et des services grâce à des informations associées, de manière à satisfaire les exigences du client » (Colin, 2005). Néanmoins, au cours des années 2000, le management logistique suscite toujours de nombreux questionnements théoriques et pratiques. Sa définition même reste discutée et il est encore confronté au dilemme " logique d'exploitation " vs. " logique d'exploration ». Face à ce constat et parce que comprendre la logistique, c'est aussi comprendre les logiques d'acteurs, des réflexions d'ordre conceptuel, orientées davantage vers la question de la prise de décision dans le contexte des chaînes logistiques multi-acteurs, sont poursuivies.

Compte tenu de l'enjeu que représente la maîtrise des flux de produits et d'information associés pour les entreprises, quel que soit leur secteur d'appartenance, les décisions logistiques se présentent peu à peu comme un terrain d'investigation idéal pour comprendre l'émergence d'une vision de la décision associant « logique d'exploitation » et " logique d'exploration ». À partir des travaux de Simon $(1958,1976)$ et de March (1991) sur la rationalité limitée et la rationalité procédurale, des travaux donnent à voir comment l'analyse s'est progressivement déplacée d'une rationalité essentiellement substantive à une rationalité résolument procédurale qui met davantage en lumière la dimension politique des démarches logistiques fondée sur le jeu des acteurs. Ils soulignent en quoi la démarche logistique se situe désormais à l'articulation entre optimisation et innovation, entre exploitation et exploration (Fulconis et Paché, 2011 ; Fulconis et Kacioui-Maurin, 2013). Ces avancées ne visent pas à contester le paradigme décisionnel dominant en matière de logistique, lequel repose sur une approche tactico-technique issue des sciences de l'ingénieur, de la recherche opérationnelle et de l'art militaire. Leur idée est principalement de souligner, pour les sciences de gestion et du management, l'intérêt de rapprocher rationalité substantive et rationalité procédurale, en montrant que chacune d'elles obéit à des contextes décisionnels précis. Les responsables, opérationnels comme stratèges, y sont invités à davantage se concerter dans les prises de décision, tant au sein d'une entreprise qu'entre une entreprise et ses partenaires économiques d'une même chaîne logistique (Lavastre et al., 2016 ; Fulconis et al., 2019).

\section{Pouvoir, conflit, coopération : les apports de l'approche organisationnelle}

Dès l'instant où la chaîne logistique est perçue dans une logique d'acteurs, que ceux-ci soient individuels ou organisationnels, la question des manœuvres stratégiques qu'ils mettent en œuvre pour capter à leur avantage la valeur créée se pose avec insistance. Pour y parvenir, le registre du pouvoir d'influence va être le plus souvent utilisé, comme le soulignent clairement les travaux de Cox et al. (2001). Une telle dimension essentielle n'est pas prise en compte par l'approche ingénierique, alors même qu'elle impacte directement les chaînes logistiques. Ceci n'est pas étonnant en soi car l'agent fait plus ou moins référence à un système holonique fondé sur de simples entités cyber-physiques connectées entre elles. La question du pouvoir s'appuie sur les travaux de French et Raven (1959), dont le mérite est d'avoir clairement identifié les cinq sources de pouvoir à la disposition d'un acteur : 
quatre sources de nature non coercitive (récompense, expertise, légitimité et valeur de référence), et une source de nature coercitive (sanction). Les sources de pouvoir de nature non coercitive et coercitive font directement écho au management des chaînes logistiques :

- La récompense. En massifiant les flux à destination du réseau de magasins et de drives sur quelques entrepôts et plates-formes logistiques, un distributeur offre à l'industriel l'opportunité de réaliser des gains de productivité sur ses opérations de transport, de manutention et de stockage. Il est donc en situation de le "récompenser ", même si la rétrocession partielle sous forme de remises pour passage par entrepôt réduit le montant total de la rétribution, autrement dit de la récompense.

- L'expertise. Un industriel possède habituellement des compétences industrielles et commerciales en packaging des produits (optimisation des unités de conditionnement et d'emballage). La normalisation et le compactage des emballages de nombreux produits de grande consommation ont ainsi conduit à une meilleure gestion dans l'approvisionnement et la gestion des linéaires qui confère à l'industriel un vrai pouvoir d'expertise vis-à-vis du distributeur.

- La légitimité. Les compétences logistiques accumulées - et reconnues - par un membre de la chaîne logistique finissent par lui assurer un certain " prestige ". Ainsi, la mutualisation des opérations logistiques conduite par certains PSL les rend légitimes aux yeux des industriels et des distributeurs dans une politique de réduction de l'impact écologique des activités d'acheminement des produits. Les PSL démontrent ainsi des qualités dans le pilotage des flux logistiques qui leur confèrent une réelle autorité.

- La valeur de référence. Permettre à un transporteur d'être identifié comme partie prenante d'une organisation logistique performante crée une source de pouvoir. L'un des exemples illustratifs est celui des 4PL (fourth party logistics). Un 4PL va effectivement mobiliser des partenaires, dont des transporteurs, pour exécuter sa prestation. Lorsque cette dernière est reconnue comme exceptionnelle par les chargeurs, ledit 4PL devient une valeur de référence pour ses partenaires.

- La sanction. Un membre de la chaîne logistique peut être persuadé qu'un autre membre (dominant) est apte à le priver d'un certain nombre d'avantages, financiers ou non financiers. Par exemple, un distributeur disposera d'un pouvoir évident sur un industriel si ce dernier est convaincu que ses produits peuvent être retirés de l'assortiment - en magasin ou en ligne - en cas de ruptures d'approvisionnement répétées, entraînant alors une perte importante de chiffre d’affaires.

L'exercice du pouvoir, notamment dans sa déclinaison coercitive, est à l'origine de la plupart des situations conflictuelles rencontrées. Le conflit dans les chaînes logistiques multi-acteurs et, plus largement dans les systèmes inter-organisationnels, a fait l'objet de nombre de recherches académiques. Le conflit y est appréhendé comme résultant d'une situation de tensions (paradoxales) dans le processus de partage de la valeur créée. Une telle tension découle du fait qu'un membre d'une chaîne logistique, par exemple un PSL, réalise qu'un autre membre, par exemple son client distributeur, profite de son pouvoir de légitimité et/ou de sanction pour imposer une organisation qui empêche le PSL d'atteindre ses propres objectifs. Par exemple, le distributeur exigera de son PSL qu'il mette en œuvre un contrat 
logistique dédié (ou exclusif) alors que ce même PSL pourrait aisément, et économiquement, procéder à une mutualisation des ressources d'entreposage et de transport avec d'autres distributeurs. L'intensité du conflit est dès lors, en grande partie, fonction de deux éléments :

- Les sujets de dissension. Ils portent, d'une part, sur la formalisation des domaines respectifs de compétences des membres de la chaîne logistique ; les désaccords renvoient, par exemple, à l'acteur ayant l'expertise ultime en matière d'équipement d'un entrepôt ou d'une plate-forme logistique, avec la présence (ou non) d'investissements idiosyncrasiques faiblement redéployables au sens de Williamson (1991). Ils portent, d'autre part, sur la répartition des processus de décision entre les membres de la chaîne logistique; les désaccords renvoient, par exemple, à l'acteur qui doit disposer des prérogatives de déclenchement des réapprovisionnements.

- L'absence de visions convergentes. Dans un environnement perçu comme stable, les divergences d'objectifs entre membres d'une chaîne logistique sont le plus souvent mineures, tandis que dans un environnement turbulent, les divergences vont susciter des stratégies de résistance ou de contournement au pouvoir du membre dominant. La crise de la Covid-19 a ainsi souligné combien la gestion des processus logistiques donnait lieu à des perceptions opposées entre des industriels à la recherche d'une sécurisation de leurs approvisionnements en matières et composants, et des fournisseurs cherchant à tout prix à acheminer leur production, y compris en trouvant des acheteurs alternatifs et en utilisant les chaînes logistiques les plus résilientes.

Compte tenu des coûts potentiels induits par des conflits dysfonctionnels, un nouveau discours émerge pour vanter les vertus de la coopération. Cependant, si le terme de coopération est souvent mobilisé, il s'avère excessif d'en généraliser l'usage car les enjeux de pouvoir restent très présents. S'intéressant au domaine de la logistique, Paché (2002) préfère d'ailleurs évoquer la présence simultanée de logiques de compétition et de coopération, et quand cette dernière émerge, il s'agit sans doute plus d'une " coordination améliorée des flux » que d'une véritable stratégie coopérative. La coordination cherche à améliorer la gestion des opérations entre membres de la chaîne logistique, ce qui nécessite une relative compatibilité entre leurs modes de fonctionnement. La coopération va au-delà de la simple interdépendance puisque les membres de la chaîne logistique vont définir ensemble un projet productif de long terme dont la concrétisation exige un certain niveau de confiance, à la fois dans les compétences techniques et managériales des autres parties, et dans le respect loyal de leurs engagements (Kwon et Suh, 2004 ; Christopher, 2016).

L'une des évolutions les plus significatives dans une approche coopérative des chaînes logistiques est sans doute le développement de la technologie blockchain, fondée sur des bases de données cryptées et distribuées qui constituent le " registre numérique " de toutes les transactions réalisées entre les membres (Wang et al., 2019). Ce " registre numérique » est accessible sur plusieurs centaines de milliers d'ordinateurs, mais il n'est pas obligatoire de le conserver en un seul endroit comme dans le cas des data warehouses. L'échange d'informations étant renforcé et facilité, dans une logique de traçabilité totale, des zones de coopération peuvent se développer. Le partage des données s'avère plus aisé, ce qui apporte de 
ce fait plus d'efficacité dans le pilotage de la chaîne logistique, notamment en réduisant le bullwhip effect selon lequel une faible variation de la demande en magasin s'amplifie au fur et à mesure qu'elle remonte la chaîne logistique vers les fournisseurs (Lee et al., 1997), rendant difficile leur activité de prévision de la production. Cet effet a deux origines principales : les achats par à-coup liés aux politiques promotionnelles des distributeurs, et les ajustements de prévision des ventes à chacune des étapes de la chaîne logistique.

L'organisation et le pilotage des flux de produits constituent un terrain de coopération complémentaire. Les développements les plus récents en matière de supply chain management (SCM) mettent particulièrement l'accent sur la nécessaire perception convergente de la réalité par les entreprises nouant des relations logistiques durables dans le processus de mise à disposition des produits à un client intermédiaire ou final. Sur un plan conceptuel, le SCM fait référence à un niveau de coordination situé entre le marché et la hiérarchie, pour reprendre la terminologie williamsonienne, autrement dit une coordination fondée sur une vision partenariale (ou hybride). Sur un plan opérationnel, le SCM introduit des ruptures majeures par rapport au mode de pensée traditionnel fondé sur la transaction plutôt que la relation : sont ainsi privilégiés désormais des contacts fréquents entre les différents membres de la chaîne logistique, un partage à long terme des risques, des bénéfices et des informations, ou encore une gestion globale des stocks le long de la chaîne (Stock et al., 2010).

Il est possible de distinguer deux niveaux de collaboration dans le pilotage des flux de produits : un niveau opérationnel et un niveau stratégique. De la même manière, la réalité des stratégies coopératives est parfois différente des idéaltypes que la littérature académique diffuse largement. Au niveau opérationnel, des coopérations poursuivent un objectif de simple optimisation de la logistique, par exemple la mise en place de centres de consolidation entre un industriel, un distributeur et un PSL pour accélérer les flux en transit dans un contexte de stock 0 en magasin. Au niveau stratégique, en revanche, les coopérations vont générer des changements organisationnels profonds, par exemple avec la constitution d'équipes projet transversales aux membres d'une chaîne logistique. Cela ne signifie pas nécessairement que les régimes de pouvoir étudiés par Cox et al. (2001) n'ont plus lieu d'être. Bien au contraire, des projets coopératifs seront sans doute impulsés par un leader qui s'appuiera sur son pouvoir d'expertise, de valeur de référence ou de récompense afin de susciter l'adhésion à son projet. C'est donc dire si la compréhension des enjeux logistiques mobilise une grande diversité de champs disciplinaires structurant les sciences de gestion et du management.

\section{Le paradigme managérial est-il audible?}

II semble entendu que, pour en saisir toutes les facettes, le « fait logistique " ne peut se réduire à la perspective holonique propre au paradigme optimisateur ou, pour reprendre les métaphores de l'organisation de Morgan (1986/2006), à une logique cybernétique d'interaction entre de simples " entités ». Au contraire, le paradigme managérial se fonde sur des jeux politiques entre acteurs dont l'analyse conduit à une exploration de nature organisationnelle des processus de création et de répartition 
de la valeur. De ce point de vue, réduire le management des chaînes logistiques à l'optimisation - sous contrainte - des opérations physiques liées au flux de produits ne peut être satisfaisant, surtout en les considérant indépendamment les unes des autres (transport, entreposage, préparation des commandes, etc.). La logistique se positionne au contraire à la croisée de multiples champs disciplinaires qui conduisent à envisager une perspective systémique et holistique que Tixier et al. (1996) ont identifié et formalisé très tôt. Des travaux plus récents mettent d'ailleurs l'accent sur l'intérêt de cette perspective pour les recherches menées " en ॥ logistique, qu'il faut distinguer des recherches pour lesquelles la logistique serait un simple «terrain d'études " (Fabbe-Costes et al., 2013). Malgré la richesse d'une telle perspective, il n'est pas sûr qu'elle rende aisée la démarche de publication des chercheurs dans les meilleurs supports.

\section{La logistique à la croisée des champs disciplinaires}

Le management performant d'une chaîne logistique est essentiel pour assurer à ses membres un niveau suffisant de rentabilité, y compris en tenant compte des disruptions générées par l'environnement. Ainsi, la crise sanitaire de 2020 a fait comprendre à nombre de décideurs et hommes politiques que la mise en œuvre d'un réseau de fournisseurs mondiaux n'était pas sans effet négatif en matière de vulnérabilité, tout particulièrement au niveau des approvisionnements (Fulconis et Paché, 2020). Réduire les risques de rupture en préparant des solutions alternatives, au cas où un axe logistique majeur serait interrompu, s'est présenté comme une urgence absolue, et pourtant souvent négligée. Plus largement, le management performant d'une chaîne logistique renvoie à des problématiques de planification des ressources et capacités d'une part, de compréhension des comportements d'achat en fonction des gammes de produit d'autre part. L'évaluation au plus près de la demande permet en effet aux entreprises d'améliorer le fonctionnement des processus logistiques, en évitant un excès ou une pénurie de stocks, et en facilitant l'ajustement des plans directeurs de production. C'est donc dire si la logistique est naturellement, et étroitement, en interaction avec le marketing.

Pour maximiser le retour sur investissement des dépenses de marketing, il est bien sûr important de se référer à la rentabilité des clients et des produits, afin de s'assurer que les efforts commerciaux sont bien dirigés vers les clients et les produits les plus attractifs. Pour cela, les entreprises disposent d'indicateurs permettant aux équipes de gérer au mieux un processus décisionnel fondé sur la définition de zones géographiques, de gammes de produits, ou encore de canaux de distribution à privilégier. Ceci conduit à pouvoir réagir avec agilité aux conditions changeantes du marché, en consolidant en temps réel les données provenant du terrain, notamment avec l'aide de la big data analytics (Dubey et al., 2019). Encore faut-il que les ressources et capacités logistiques soient disponibles pour faire face aux nouvelles exigences. Le secteur de la distribution est un bon exemple des difficultés rencontrées en la matière. Ainsi, alors que la montée en puissance de la vente en ligne des produits alimentaires a été identifiée comme une tendance lourde dès le milieu des années 2000, témoignage d'un changement profond dans les comportements d'achat, les plus grands distributeurs n'ont jamais vraiment su développer une 
logistique du dernier kilomètre suffisamment efficace pour livrer jusqu'au domicile du consommateur, conduisant au développement d'une solution dégradée : le drive.

Le développement de la vente en ligne souligne une autre interaction clé : celle de la logistique et des systèmes d'information. Certes, notamment depuis les travaux séminaux conduits par Fabbe-Costes (2000) il y a vingt ans de cela, on sait combien les systèmes d'information occupent une place centrale dans le management des chaînes logistiques afin d'assurer l'enregistrement, l'analyse, la gestion et l'utilisation des données tout au long du flux de produits. De l'efficacité des systèmes d'information découlent des processus commerciaux performants pour satisfaire la demande, à la fois en termes de planification et prévision, de gestion des stocks, d'exécution des commandes, de traçabilité des produits, de maintenance et de suivi des performances. Avec la vente en ligne, le consommateur est connecté aux systèmes d'information, et pénètre ainsi une partie du back office logistique, longtemps resté secret - voire mystérieux - à ses yeux. Pour les entreprises, il s'agit alors de sélectionner les technologies, notamment logicielles, les plus adaptées pour maintenir un niveau élevé de réactivité face à une demande volatile, dans le respect d'une allocation optimale des ressources financières. En l'absence d'ajustement rapide des systèmes d'information, c'est la performance logistique qui est menacée, et par conséquent le passage réussi à l'ère du numérique.

Enfin, le management des chaînes logistiques interpelle directement la gestion des ressources humaines, tant il est vrai que les transformations en œuvre dans la façon d'organiser le pilotage des flux interagit avec les pratiques de recrutement, de formation, de dotation en personnel et de suivi des performances des salariés (McAfee et al., 2002). En effet, les décisions relatives aux ressources humaines doivent être alignées sur les besoins à court et à long terme de l'organisation, et rigoureusement alignées sur les objectifs de production et de distribution. Le principe même des chaînes logistiques est de solidariser des maillons, sachant que la défaillance, même ponctuelle, de l'un d'eux menace immédiatement la performance globale. Les politiques salariales, ainsi que les décisions relatives aux primes et aux plans de rémunération (fixe et variable), sont habituellement gérées au niveau du salarié ou d'un groupe de salariés pour chaque entreprise membre de la chaîne logistique, des salariés répartis souvent sur plusieurs pays aux législations différentes. On conçoit sans peine des divergences majeures de traitement dont l'impact pourrait être significatif, par exemple en matière de blocage d'une unité industrielle et logistique compte tenu de conditions de rémunération ou de travail jugées inacceptables. L'un des cas les plus connus est celui des constructeurs automobiles dont l'arrêt d'une chaîne de production représente environ 5000 euros par minute en cas de rupture d'approvisionnement en pièces et composants à la suite d'un mouvement de grève chez l'un des fournisseurs.

À l'évidence, d'autres champs disciplinaires des sciences de gestion et du management entrent en résonance avec la logistique, notamment lorsqu'il s'agit d'aborder la question des choix stratégiques effectués par des entreprises, d'un côté, par exemple en matière d'externalisation, de délocalisation ou, à l'inverse, de retour à la proximité, et la performance qui en découle, de l'autre. Les outils et démarches analytiques du contrôle de gestion s'avèrent ici essentiels pour identifier les informations clés sous forme de KPI synthétisés dans des tableaux de bord de type cockpit (Cekerevac, 2013). Une approche contrôle doit ainsi permettre 
d'apporter des réponses relatives au calcul des coûts résultant de l'exécution des opérations logistiques, en interne ou en externe, la relation entre le coût et le mode de transport choisi, ou encore la relation entre l'intégration des systèmes d'information, la flexibilisation de la production et la performance commerciale. L'une des tendances les plus lourdes à ce niveau est le développement des réflexions sur la supply chain finance, dont l'objectif est de réduire le coût du capital afin d'optimiser le fonds de roulement et de rationaliser le processus de paiement pour les membres d'une chaîne logistique (Viviani et Vo, 2016 ; Xu et al., 2018). Concrètement, une institution financière intervient ici pour offrir des coûts de financement plus attractifs à un fournisseur, en fonction de la notation de crédit de l'acheteur, généralement meilleure, ce qui favorise une meilleure santé financière et une capacité accrue à faire face aux aléas de la conjoncture.

Au final, la compréhension de la dynamique des chaînes logistiques se nourrit des apports de plusieurs champs disciplinaires, mais les nourrit également en retour pour élargir leurs propres analyses. On ne peut ainsi imaginer ce que pourraient être les recherches sur le sustainable management sans la prise en compte des enjeux logistiques, particulièrement en ce qui concerne la logistique urbaine durable (Taniguchi, 2014). Cette dernière se positionne au demeurant comme l'une des composantes de l'action publique, et renvoie à des cadres conceptuels explorés de longue date en management public. De manière symptomatique, lorsque la question de l'organisation de la vaccination de masse s'est posée en décembre 2020 aux autorités politiques pour sortir de la Covid-19, la dimension logistique a instantanément été présentée comme critique pour transporter, stocker et délivrer les vaccins Pfizer / BioNTech et Moderna compte tenu d'une chaîne du froid atteignant des températures (basses) inédites. Cette " infusion " de la logistique dans l'ensemble des sciences de gestion et du management fait sans doute sa richesse en tant que démarche transversale, mais aussi sa faiblesse en termes de visibilité académique : la logistique étant partout, où est-elle vraiment? II s'agit là d'un défi majeur pour les chercheurs qui se réclament d'elle quand il s'agit d'identifier les supports les plus adaptés, et les plus réceptifs, pour publier leurs travaux.

\section{Diffuser les recherches en logistique : un "parcours du combattant»}

Des doctorants ou de récents docteurs en sciences de gestion et du management de 2022 peuvent-ils imaginer un monde académique dans lequel le " ranking » des revues n'existait pas ? Un monde dans lequel l'évaluation des dossiers, notamment lors de recrutements, passait quasi-exclusivement par une lecture des travaux en commission pour en juger la rigueur et la pertinence, tant conceptuelle que managériale ? Une telle réalité semble aujourd'hui à peine croyable alors même que les classements de revues se sont multipliés à travers le monde, en s'appuyant pour cela sur un ensemble de critères d'évaluation, dont l'un des plus connus est l'impact factor. $S^{\prime}$ il fallait identifier une sorte de point de bascule, il se situerait en France entre les années 2000 et 2005, avec une volonté affichée de plusieurs institutions et associations académiques de se doter d'outils de mesure fiables et non contestables de la performance d'un chercheur en sciences de gestion et du management, en s'alignant au demeurant sur des pratiques en vigueur dans les " sciences dures ». Depuis lors, pour le meilleur et pour le pire, les classements de revues sont devenus un étalon 
universel, la valeur la plus sûre pour identifier les " hauts potentiels ", voire un outil majeur de communication pour les chercheurs eux-mêmes. Il suffit de consulter les réseaux sociaux pour découvrir régulièrement la mise en scène, par un collègue, de sa dernière publication " dans une revue de rang $A$ ", un peu comme les chasseurs de bartavelles de Marcel Pagnol, dans La gloire de mon père, exhibaient fièrement leurs trophées sur la place du marché !

Il n'en reste pas moins que le monde étant ce qu'il est, publier dans les revues les mieux cotées apparaît désormais comme un point de passage obligé dans la carrière. Les chercheurs en management logistique n'échappent pas à cette règle structurante. Ils ont par conséquent en tête l'existence de classements possibles, et selon la voie choisie (business school ou université), une stratégie personnelle bien comprise s'appuiera sur la publication d'articles dans des revues d'un ou deux classements cibles. Si l'on s'en tient ici à l'instance d'évaluation et de promotion qu'est la section 06 du CNU, le classement de référence est désormais celui établi par la FNEGE (juin 2019 pour le dernier en date). II hiérarchise des revues en sciences de gestion et du management selon cinq catégories, en partant des revues jugées exceptionnellement sélectives $\left(1^{*}\right)$ pour arriver pas à pas aux revues de sélectivité satisfaisante répondant aux principes conventionnels d'arbitrage (4). Nul ne conteste aujourd'hui la rigueur du processus de classement de la FNEGE, qui associe 24 associations académiques, et s'appuie sur neuf classements préexistants (en France, en Allemagne, en Australie, aux Pays-Bas et au Royaume-Uni) et cinq indices bibliométriques (dont le fameux impact factor du Web of Science). Pour tenir compte du fait que les revues francophones restent rarement intégrées dans les classements internationaux, une procédure parallèle y est conduite en s'appuyant notamment sur le $h$ index. Enfin, chacune des revues retenues est rattachée à l'un des 16 champs disciplinaires identifiés, dont la logistique.

L'analyse factuelle du classement FNEGE 2019, qui n'introduit d'ailleurs pas de rupture majeure par rapport aux classements antérieurs, permet de parler d'un véritable " parcours du combattant » pour le chercheur en logistique s'inscrivant dans le paradigme managérial. Si l'on prend en compte les seules revues classées $1^{*}$, 1 et 2 (soit les revues dites de " rang A »), 178 sont répertoriées, dont 19 pour la logistique (un peu plus de $10 \%$ ), ce qui n'est pas un mauvais chiffre en soi. En revanche, si l'on se réfère à la ligne éditoriale des 19 revues, telle qu'elle transparaît dans le positionnement affiché par le comité de rédaction et dans les articles qui y sont publiés, la dominante reste majoritairement ingénierique (tableau 2). Autrement dit, alors même que l'approche organisationnelle est reconnue, notamment dans l'univers des professionnels, comme essentielle pour comprendre la dynamique des chaînes logistiques dans un monde globalisé marqué par des disruptions majeures, le paradigme ingénierique reste privilégié. Plus inquiétant encore, ce paradigme est exclusivement anglo-saxon puisqu'aucune revue francophone se retrouve dans le " club des 19 ». Il faut atteindre le rang 3 (dit " rang B ») pour retrouver, parmi les 14 revues présentes, une revue francophone de référence : Logistique \& Management. En bref, rien n'est simple pour le chercheur en management logistique voulant avancer dans la carrière, qu'il soit jeune ou confirmé... puisqu'au final, le classement FNEGE 2019 ne reconnaît que 37 revues dans le champ, dont près de la moitié relevant du paradigme ingénierique. 
Tableau 2 : Revues de rang A relevant du champ de la logistique dans le classement FNEGE 2019

\begin{tabular}{|c|c|c|c|c|}
\hline \multirow[b]{2}{*}{ Revues } & \multirow{2}{*}{$\begin{array}{c}\text { Rangs } \\
\text { FNEGE } \\
2019\end{array}$} & \multicolumn{3}{|c|}{ Lignes éditoriales } \\
\hline & & $\begin{array}{l}\text { Dominante } \\
\text { ingénierique }\end{array}$ & $\begin{array}{l}\text { Dominante } \\
\text { managériale }\end{array}$ & Équilibrée \\
\hline Management Science & $1^{*}$ & & & \\
\hline $\begin{array}{r}\text { European Journal } \\
\text { of Operational Research }\end{array}$ & 1 & & & \\
\hline $\begin{array}{r}\text { International Journal } \\
\text { of Production Economics }\end{array}$ & 1 & & & \\
\hline Journal of Operations Management & 1 & & & \\
\hline $\begin{array}{r}\text { Manufacturing \& Service Operations } \\
\text { Management }\end{array}$ & 1 & & & \\
\hline Operations Research & 1 & & & \\
\hline Production \& Operations Management & 1 & & & \\
\hline Annals of Operations Research & 2 & & & \\
\hline Decision Sciences & 2 & & & \\
\hline $\begin{array}{r}\text { International Journal of Operations } \\
\text { \& Production Management }\end{array}$ & 2 & & & \\
\hline $\begin{array}{l}\text { International Journal of Physical } \\
\text { Distribution \& Logistics Management }\end{array}$ & 2 & & & \\
\hline $\begin{array}{l}\text { International Journal } \\
\text { of Production Research }\end{array}$ & 2 & & & \\
\hline $\begin{array}{r}\text { International Journal } \\
\text { of Project Management }\end{array}$ & 2 & & & \\
\hline Journal of Business Logistics & 2 & & & \\
\hline Journal of Supply Chain Management & 2 & & & \\
\hline $\begin{array}{r}\text { Journal of the Operational } \\
\text { Research Society }\end{array}$ & 2 & & & \\
\hline Production Planning \& Control & 2 & & & \\
\hline Supply Chain Management & 2 & & & \\
\hline Transportation Research Part E & 2 & & & \\
\hline
\end{tabular}

Fort heureusement, comme nous l'avons souligné précédemment, l'analyse managériale du fonctionnement des chaînes logistiques se nourrit des apports d'autres champs disciplinaires, et elle les nourrit en retour de ses propres analyses. Cette position privilégiée de marginal sécant permet de trouver des terres d'accueil dans des supports hors du strict " territoire " de la logistique. Bien évidemment, toute la difficulté pour le chercheur sera alors de s'approprier les codes explicites et implicites du support visé, voire les habitus d'une communauté académique au sens de Bourdieu (1980), autrement dit le "système de dispositions réglées » en matière d'identification des courants majeurs de recherche, d'auteurs incontournables à connaître et à citer, des méthodologies les plus appropriées... mais aussi, et souvent, du style argumentatif propre à une discipline donnée ! Ceci explique sans doute le fait que nombre de chercheurs en management logistique se tournent vers des revues de type " généraliste " pour défendre leur position de marginal sécant, difficilement 
audible par des supports ultraspécialisés. Le succès de plusieurs dossiers spéciaux de la Revue française de gestion consacrés à la logistique et au SCM, par-delà la publication régulière d'articles hors dossiers, témoigne de la reconnaissance bienvenue, par une revue de rang 2 , de travaux œuvrant pour une approche décloisonnée et innovante des processus de création de valeur.

\section{Conclusion}

Nul ne conteste que la logistique contemporaine trouve ses racines dans les progrès de la recherche opérationnelle à partir des années 1930, et plus encore, pendant la Seconde Guerre mondiale, des progrès qui donneront lieu rapidement à la diffusion du savoir dans des revues spécialisées dès le début des années 1950 (la revue Operations Research est lancée aux États-Unis en 1952). Comme on le sait, la recherche opérationnelle s'appuie sur des techniques mathématiques, statistiques et algorithmiques de résolution de problèmes pour aider à la prise de décision. On doit à Charles Babbage (1791-1871) la première incursion dans le domaine du transport puisque ses recherches sur l'optimisation du coût d'acheminement et de tri du courrier ont abouti à la création du système postal britannique moderne en 1840 . C'est toutefois l'opération Overlord, autrement dit le débarquement de Normandie du 6 juin 1944, qui marque le véritable triomphe de la recherche opérationnelle appliquée à la logistique, même s'il ne faut pas oublier qu'au Royaume-Uni, une équipe de scientifiques s'est évertuée, pendant le conflit, à appliquer des techniques pour conduire à l'utilisation la plus efficace possible de ressources militaires limitées.

L'opération Overlord témoigne d'une optimisation des flux sur une échelle jamais connue jusqu'alors. Elle symbolise aussi et surtout la capacité des États-Unis à mobiliser les compétences les plus avancées en matière de recherche opérationnelle, notamment en se tournant vers des universitaires spécialisés comme Georges Doriot, professeur à Harvard, qui devient en 1941 le directeur du département de planning militaire de l'US Army avec le grade de lieutenant-colonel. Il est vrai que l'enjeu est majeur puisqu'il s'agit alors d'organiser, depuis le continent nord-américain, une attaque surprise des forces nazies en Europe en déplaçant jusqu'aux plages de Normandie 150000 hommes le jour J, puis 850000 hommes après le jour J, accompagnés de 5000 navires et 11000 avions. Une fois la Seconde Guerre mondiale terminée, l'industrie américaine accueillera massivement les spécialistes de recherche opérationnelle, désormais démobilisés, pour tirer bénéfice de leurs compétences et mettre en œuvre les optimisations logistiques indispensables en vue d'accompagner la croissance quantitative des marchés de consommation.

Face à un tel héritage, on peut comprendre aisément que le management des chaînes logistiques ait été durablement marqué par une vision ingénierique dominante. Si cette dernière ne doit pas être reniée, il reste à ne pas la considérer comme la seule manière de penser le pilotage des flux. Bien au contraire, comme nous l'avons indiqué dans le chapitre, une approche managériale s'intéressant aux logiques d'acteurs s'avère indispensable pour comprendre les enjeux logistiques en présence. Dans un monde globalisé soumis à des turbulences récurrentes, dont la pandémie de Covid-19 n'est que l'illustration la plus récente, la question qui se pose est sans doute celle de l'usage de la logistique comme arme dans la compétition. 
Force est d'admettre qu'opter pour une simple vision holonique d'entités cyber-physiques - ou agents - interconnectés ne peut rendre compte de cette " épaisseur » stratégique et organisationnelle, pourtant essentielle. La rupture paradigmatique sous-tendue ne pourra se concrétiser qu'à une seule condition : abandonner une fascination mortifère pour la recherche opérationnelle vue comme fin en soi, et non comme un ensemble d'instruments au service de la décision managériale. 


\section{Perspectives logistiques pour le système de santé \\ Enjeux technologiques et durables}

Blandine Ageron, Omar Bentahar et Smail Benzidia

Le secteur de la santé, particulièrement le système hospitalier, sont un terrain d'études de première importance pour de nombreux travaux, que ce soit en sciences économiques, en sociologie, en géographie et en sciences de gestion et du management. Ils donnent lieu à des recherches variées et à des valorisations contributives dans ces différents champs de recherche. La logistique et le management de la chaîne logistique (ou supply chain management [SCM]) s'inscrivent dans cette tradition depuis plus de trente ans. Nous nous proposons dans le présent chapitre de rappeler le contexte évolutif du secteur de la santé, de présenter les caractéristiques de la logistique et du management de la chaîne logistique hospitaliers, ainsi que de détailler les récents enjeux auxquels ils sont confrontés.

\section{Un contexte d'étude actuel et pertinent}

Depuis plusieurs années, le système hospitalier subit des évolutions importantes qui l'entraîne progressivement dans une crise profonde. Parmi les causes importantes de cette crise, citons une réduction des capacités d'hospitalisation, une très forte croissance des prises en charge aux urgences, une certaine dégradation des conditions de travail et une contraction globale des moyens. Pour sortir de la crise, le système hospitalier se voit traversé par un ensemble de mutations importantes dont les objectifs peuvent apparaître contradictoires (Juven et al., 2019). Les nombreuses réformes menées depuis le début des années 1990 constituent sans aucun doute la mutation la plus importante et s'expliquent par la volonté des Pouvoirs publics de réduire les coûts de fonctionnement de l'hôpital.

Une première réforme a consisté à modifier la gouvernance des hôpitaux et plus particulièrement l'exercice de la tutelle par les créations successives des agences régionales d'hospitalisation puis de santé en 1996, puis celle des agences régionales 
de la santé en 2009. Une seconde réforme s'est intéressée au mode de financement des établissements hospitaliers français, qu'ils soient privés ou publics, passant successivement du " prix journalier », à la " dotation globale " et à la " tarification à l'activité » (T2A), en 2003. Cette dernière consiste à rémunérer les établissements de santé selon leur activité médicale effective. Le but de la réforme est ainsi d'obtenir une plus grande transparence quant à la gouvernance des hôpitaux et d'assurer une répartition plus équitable des ressources financières entre les établissements, et ce, en se fondant sur la nature et le volume - réel - des activités réalisées. Elle est également pensée comme un moyen d'inciter les agents hospitaliers à se réformer eux même et à revoir leur routines et rôles dans le système hospitalier de soin. D’ailleurs, force est de constater que la qualité des soins et les conditions de travail, les deux autres mutations importantes du secteur, en découlent.

La qualité des soins et son amélioration via un processus de normalisation sont au cœur d'une deuxième mutation majeure, amorcée à partir du milieu des années 1980, par le secteur hospitalier. La spécialisation technique de l'hôpital s'est accompagnée de tentatives visant à standardiser et normaliser l'offre de soins. La publication d'un rapport annuel sur la qualité des soins dans les établissements, la création en 1989 de l'Agence nationale pour le développement de l'évaluation médicale (ANDEM), ou encore la création en 2004 de la Haute autorité de santé (HAS), sont autant d'éléments de preuve d'une volonté de la puissance publique de s'emparer de la question sensible de la qualité des soins.

Enfin, la troisième mutation importante concerne le management des ressources humaines et vise à améliorer les conditions de travail dans un contexte de pénurie annoncée de personnels, notamment de personnels soignants. Plusieurs éléments participent de cette nécessité de repenser les conditions de travail : un manque d'attractivité du secteur et des métiers, les départs prématurés de personnels en poste vers de nouveaux horizons professionnels, une difficulté à stabiliser les effectifs et des absences répétées et/ou de longue période. Les établissements hospitaliers ont dû réagir et prendre des mesures pour gérer cette menace qui provoque des désorganisations importantes tant au niveau du système de soin que du fonctionnement global (Estryn-Behar, 2008). Depuis le début des années 2010, la puissance publique a ainsi multiplié les initiatives autour du dialogue social ou encore de la qualité de vie au travail.

Les différentes réformes et mutations, rapidement exposées ci-dessus quant à leurs dimensions essentielles, reposent sur un ensemble de dispositifs qui visent à mettre en concurrence les hôpitaux et qui empruntent au secteur industriel des méthodes de gestion et de pilotage de la performance. Le but final est de maîtriser les dépenses tout en améliorant la qualité du service rendu. Cette double injonction appelle une restructuration de l'organisation interne des hôpitaux et une redéfinition des rôles des acteurs (Ageron et al., 2018 ; Sampieri-Teissier et Livolsi, 2019). Elle repose sur le développement et le renforcement de compétences et la mise en œuvre de projets transversaux favorisant la coopération entre les différents acteurs internes et externes à l'établissement (Beaulieu et al., 2014).

Dans ce contexte, la logistique et le SCM semblent être une opportunité pour garantir cette vision managériale et transversale nécessaire à la maîtrise des coûts et à l'optimisation des flux. Leur capacité à transcender les séparations entre les 
métiers, organisations et logiques fonctionnelles favorise le décloisonnement et la coopération. La supply chain de la santé peut être définie

comme étant une stratégie de management agile des flux patients, physiques (pharmacie, linge, restauration, déchets) et informationnels, en allant du fournisseur jusqu'aux utilisateurs finaux, afin de créer une valeur durable pour le patient (Bentahar et Benzidia, 2019).

Elle a pour mission d'étudier et d'optimiser deux types de flux physiques : les flux de personnes et les flux de matières. La section suivante présente de façon détaillée les caractéristiques de la logistique de la santé.

\section{Les caractéristiques de la logistique / supply chain de la santé}

Le secteur de la santé connaît ces dernières années une transformation organisationnelle et technologique radicale visant à réduire les coûts tout en améliorant la création de valeur pour les parties prenantes. La recherche et la pratique dans le domaine du SCM de la logistique peuvent contribuer de manière significative à l'évolution du système de la santé et à l'atteinte des objectifs stratégiques (Bentahar et Benzidia, 2019). En effet, la supply chain de la santé, reste très en retard par rapport aux supply chains des autres secteurs industriels en matière d'agilité, de robustesse et de résilience. Dès la fin des années 1990, de manière précoce, Rickles (1999) soulignait que la supply chain de la santé avait vingt ans de retard sur celles des secteurs de l'alimentaire et du commerce de détail. Aujourd'hui, nos observations confirment toujours un tel constat, différentes études indiquant des coûts logistiques élevés dans la supply chain de la santé par rapport à ceux observés dans d'autres industries (Ageron et al., 2018).

La multiplicité des flux hospitaliers, les contradictions entre la logique professionnelle de soin et la logique gestionnaire peuvent expliquer en partie les faibles scores de performance de la supply chain de la santé (Husson, 2009 ; Ageron et al., 2018 ; Bentahar et Benzidia, 2019). La complexité logistique est liée à la diversité des activités (métier / support), la variété des flux physiques et informationnels et la multiplicité des acteurs (Bentahar et Benzidia, 2019). En effet, le secteur de la santé comporte plusieurs flux inter-reliés et aux caractéristiques différentes : le flux patient, le flux pharmaceutique, le flux de linge, le flux de restauration et le flux de déchets. Par ailleurs, la divergence des objectifs et des intérêts des acteurs accentue la complexité de la supply chain de la santé. Nous observons, d'une part, une logique professionnelle fondée sur l'autonomie et centrée sur le soin spécifique du patient et, d'autre part, une logique administrative fondée sur le contrôle et axée sur la gestion des capacités et des services supports standardisés pour satisfaire le volume important de la demande globale des patients.

Sur le plan organisationnel, la supply chain de la santé intègre trois grandes activités : (1) les achats ; (2) le réapprovisionnement des différents services et départements en produits et matériels; et (3) la production. Cette organisation continue à évoluer afin de s'adapter à la dynamique de l'environnement et en vue d'obtenir une amélioration des indicateurs de performance. En ce sens, Beaulieu et al. (2014) intègrent une troisième activité stratégique pour la logistique : le transport. Dans le cadre de la perspective organisationnelle, nous remarquons que la logistique souffre 
d'un faible intérêt stratégique et d'un manque de "sponsorship " par le top management, limitant l'acquisition des compétences, des innovations et des investissements technologiques nécessaires au développement des bonnes pratiques logistiques (Beaulieu et Bentahar, 2021 ; Paché, 2021a). La logistique de la santé est effectivement souvent conçue dans une optique de gestion opérationnelle, et rarement dans une perspective stratégique influant sur les décisions de la direction générale (Beaulieu et al., 2020).

Le retard de la supply chain de la santé peut être rattrapé par des initiatives de digitalisation structurées (Beaulieu et Bentahar, 2021). Cependant, les études démontrant les impacts de la digitalisation dans ce contexte singulier n'en sont qu'à leurs balbutiements. De ce point de vue, il existe un réel besoin d'études et propositions indiquant comment la digitalisation peut générer des bénéfices pour la supply chain de la santé (Ageron et al., 2020). Certes, tout au long des années 2010, les acteurs du secteur ont pris conscience de l'intérêt des approches environnementales et ont décidé de s'engager dans l'implémentation de chaînes logistiques vertes. Cependant, une telle implémentation dans les hôpitaux reste limitée, alors même que la recherche permettrait de comprendre les stratégies des organisations dans l'intégration des approches environnementales, les domaines d'application, les défis à relever et les facteurs clés de leur mise en œuvre.

\section{Le digital au service de la supply chain de la santé}

Comme de nombreux secteurs, celui de la santé a bénéficié du développement progressif des technologies de l'information, qui ont favorisé la mise en place de pratiques organisationnelles innovantes. Le domaine de la santé a commencé à intégrer principalement, des outils classiques comme le dossier médical partagé (DMP) ou le dossier patient informatisé (DPI), ou même, de nouvelles technologies avancées telles que le code-barres, le GPS et la RFID, en s'inspirant de l'expérience des autres secteurs. Au cours des dix dernières années, les technologies de l'information ont également ouvert la voie à la mise en place de pratiques médicales novatrices comme la télémédecine. Malgré la diffusion des technologies de l'information dans la supply chain de la santé, leur utilisation est largement en retard comparé à d'autres industries et $n$ 'inclut pas les outils technologiques de dernière génération comme le big data, l'intelligence artificielle, la blockchain, le cloud, etc. Au-delà des contraintes budgétaires, un tel retard pourrait être expliqué par la complexité de la supply chain de la santé connue par la diversité des flux, des soins et des acteurs internes et externes (Ageron et al., 2018).

Les technologies de nouvelle génération pourraient modifier radicalement le paysage de la santé, lui permettant d'évoluer vers des organisations intelligentes ou une santé 4.0. Cela permettrait aux managers de la santé de faire évoluer le modèle traditionnel actuel, centré sur l'hôpital et le médecin, vers un modèle de gestion intelligent ciblant davantage une autogestion du parcours du patient. Bien que nous soyons conscients de la disponibilité de nombreuses technologies numériques prometteuses pour améliorer la supply chain de la santé, il est possible d'identifier tout particulièrement l'application potentielle de deux technologies interdépendantes à savoir l'Internet des objets (IOT) et le big data et l'Internet des objets (IoT) : 
- L'utilisation de la technologie big data représentent un atout considérable dans la gestion de la supply chain de la santé grâce à sa capacité à traiter, analyser et contrôler les données liées à la circulation des patients ainsi que les flux logistiques des hôpitaux. Par conséquent, l'analyse des données massives peut promouvoir des soins individuels personnalisés et garantir la qualité de soin offert aux patients. Dans le domaine des pharmacies hospitalières, par exemple, la combinaison de I'intelligence artificielle et du big data a déjà été prouvée, notamment dans la conception et le développement de nouveaux médicaments (Chen et Butte, 2016). Ainsi, grâce à la capacité démultipliée de traitement de l'information, les hôpitaux peuvent mieux planifier leurs ressources en termes de personnel nécessaire à la prise en charge des patients (médecins, infirmiers, infirmières auxiliaires), et anticiper leurs besoins en équipements et médicaments, etc.

- La supply chain de la santé pourrait également, s'appuyer sur la technologie de I'IoT pour repousser les frontières de la santé 4.0. L'loT offre plusieurs fonctionnalités clés telles que la surveillance à distance des patients atteints de maladies chroniques, mais aussi des personnes âgées et de celles nécessitant une surveillance constante pour diverses raisons (Secundo et al., 2021). L'étude conduite par Papa et al. (2020), portant sur le marché des dispositifs de soins de santé intelligents et portables, en référence au confort, à l'utilité perçue et à la facilité perçue d'utilisation, révèle que la mise en application de l'loT a apporté d'excellents résultats dans la surveillance, la détection et la prévention de la Covid-19 en Italie.

\section{Vers une supply chain verte de la santé}

Les hôpitaux sont connus pour consommer des quantités considérables de matériaux, d'énergie et d'eau. Ils génèrent également de grandes quantités de déchets, notamment toxiques, par rapport à d'autres secteurs, et ont une empreinte carbone élevée. Aux États-Unis, par exemple, les hôpitaux produisent 6600 tonnes de déchets par jour (Kaplan, 2012), et consomment plus d'énergie que tout autre type de bâtiment commercial (Mousa et Othman, 2020). Cette contribution majeure à la pollution de l'environnement engendre de nombreux risques pathologiques, pharmaceutiques, chimiques, radioactifs et sanitaires selon l'OMS. Ainsi, l'intégration d'une approche environnementale dans la supply chain des hôpitaux représente un défi considérable pour les managers de la santé. Face à une telle situation, de nombreux pays développés, conscients de l'urgence d'une démarche environnementale, encouragent les hôpitaux à s'orienter vers des pratiques vertes. En France, par exemple, un certain nombre d'hôpitaux se sont engagés dans la mise en place de pratiques respectueuses de l'environnement, mais elles restent très limitées et s'inscrivent souvent dans une approche réactive en réponse à des pressions réglementaires (Benzidia et al., 2021).

L'une des raisons des retards environnementaux dans la supply chain hospitalière peut être en partie attribuée à l'utilisation de méthodes inappropriées de gestion hospitalière, plus axée sur la prise en charge des patients et la réduction des coûts. Ainsi, il n'est pas simple d'identifier des mécanismes qui combinent une approche économique et sécuritaire en même temps que la réduction de l'empreinte environnementale (Singh et El-Kassar, 2019). Une autre raison réside dans la politique 
d'approvisionnement des médicaments et dispositifs médicaux des hôpitaux, favorisant principalement des pays lointains, et à bas coûts, comme la Chine et l'Inde. Cette politique reflète certainement les contraintes budgétaires plus importantes auxquelles sont confrontés les hôpitaux publics, mais alourdit en parallèle le bilan de leur empreinte carbone. Par conséquent, un des leviers pour la supply chain de la santé consiste à mettre en place un processus d'achat durable intégrant, par exemple, des critères de sélection de fournisseurs respectueux de l'environnement. Une telle stratégie permettrait de gérer efficacement de nombreux facteurs en amont, tels que les coûts de transport, la sécurité du carburant, la sensibilisation à l'environnement, etc., qui ont de lourdes conséquences sur les initiatives environnementales.

\section{Conclusion}

La vulnérabilité et les retards de la supply chain de la santé par rapport à d'autres supply chains industrielles et commerciales constituent une problématique majeure auxquelles les chercheurs devront répondre. Cette réponse devrait être progressive et structurée, et non aléatoire, sous l'effet de l'urgence. Les retards peuvent être comblés en partie par l'implémentation des technologies innovantes de la digitalisation et l'intégration d'approches environnementales. En effet, la digitalisation améliore l'efficience et la réactivité des processus logistiques et la valeur créée pour le client (Giannakis et al., 2019 ; Ageron et al., 2020), alors que l'innovation environnementale peut faire bénéficier l'organisation d'une réduction des coûts, ainsi que d'une amélioration des profits et de l'image sociale (Kusi-Sarpong et al., 2019 ; Benzidia et al., 2021). Durant nos investigations sur les défis de la digitalisation et de la supply chain verte, les acteurs de la santé ont souligné également leur fort intérêt pour les approches sociales de la supply chain et le besoin de propositions managériales afin de les renforcer. Ainsi, les recherches futures dans le secteur de la santé pourraient concerner les pratiques de la supply chain sociale, leurs diffusions et bénéfices pour l'hôpital.

Enfin, en raison de la crise sanitaire de la Covid-19, on doit noter une profonde prise de conscience par les décideurs politiques de la nécessité de mobiliser tous les moyens et ressources pour faire évoluer la stratégie hospitalière actuelle, fondée principalement sur la réduction des coûts. Les managers de la santé peuvent saisir une telle opportunité pour renforcer l'utilisation et l'apprentissage numérique dans la supply chain de la santé. De plus, il est également nécessaire d’accompagner les hôpitaux dans leur transition écologique en leur offrant les ressources suffisantes pour mettre en place des initiatives respectueuses de l'environnement, telles que l'éco-conception, le recyclage, l'efficacité énergétique, la conservation de l'eau, les achats écologiques et la mobilité durable. 
Se projeter 



\title{
Conseil académique - Conseil national des universités
}

\author{
Deux instances aux missions complémentaires \\ au service de la reconnaissance \\ des enseignants-chercheurs
}

Marine Legall-Ely, Jérémy Morvan, Isabelle Sauviat et Nathalie Schieb-Bienfait

Depuis 2007, avec la promulgation de la loi relative aux libertés et responsabilités des universités (dite loi LRU ou loi Pécresse du 10 août 2007), les universités françaises vivent une période de mutations, avec d'importantes réformes statutaires, dont il est parfois difficile de discerner la logique et la cohérence d'ensemble et surtout d'en cerner voire d'en mesurer les impacts à court et moyen terme sur l'enseignement supérieur et la recherche et plus particulièrement sur le métier d'enseignant chercheur. Notre propos se centre sur la loi dite "LPR » (loi de programmation de la recherche), notamment sur certains articles de cette loi, qui a été promulguée le 24 décembre 2020. Rappelons que cette promulgation a suscité de vives polémiques, comme en témoignent les nombreuses motions de sections du CNU : pétitions, appels à la grève, écrans noirs sur les réseaux sociaux, etc. La presse a parlé de son côté de "fronde contre la loi » (de Montecler, 2020).

Pourtant, cette loi avait nourri de nombreux espoirs. La communauté des chercheurs et enseignants-chercheurs convergeait sur le besoin d'une réforme de la recherche et de l'enseignement supérieur face à la diminution du nombre de postes, à la précarisation croissante des jeunes chercheurs, au manque de personnel, de support à la recherche dans les universités, à l'insuffisante visibilité de la recherche française à l'international. La loi avait ainsi l'objectif d'un rattrapage inédit sur le retard de financement de la recherche publique par rapport à de nombreux pays qui consentaient un effort supérieur depuis longtemps, menaçant la France d'un décrochage scientifique. Alors que le monde universitaire réclamait unanimement un grand plan de relance et d'investissement dans l'université et la recherche, la déception est d'autant plus grande et l'inquiétude réelle. Pour Mathias Bernard, président de 
I'université Clermont Auvergne, la LPR « n'aborde ni la question des investissements en équipements scientifiques ni celle de l'emploi. Les seules mesures de ressources humaines visent à faciliter le recrutement de contractuels. C'est une déception » (Monod, 2020). La LPR paraît non seulement très loin des attentes du monde universitaire et de la recherche, mais de surcroît, la communauté des chercheurs s'interroge : cette loi viendrait-elle empirer la situation ou figer un statu quo ?

C'est précisément ce questionnement que nous souhaitons instruire dans le présent chapitre, en nous intéressant plus particulièrement à l'article 5 de cette loi, article qui fut ajouté suite à deux amendements sénatoriaux du 29 octobre 2020. Loin du décret du 6 juin 1984 fixant les dispositions statutaires communes applicables aux enseignants-chercheurs et portant sur le statut du corps des professeurs des universités et du corps des maîtres de conférences, il rend désormais possible le recrutement des enseignants-chercheurs sans passer par la qualification du CNU, avec comme objectifs affichés d'élargir les viviers des candidats potentiels et de fluidifier l'accès aux corps des enseignants-chercheurs. De fait, le CNU, en tant qu'instance nationale élue et dédiée à l'accompagnement de la carrière des enseignants-chercheurs, se trouve remis en cause, rejoignant en écho les nombreuses critiques à charge qui lui étaient faites depuis de nombreuses années : immobilisme, perte de légitimité, frein à la pluridisciplinarité, démarche coûteuse et chronophage (Garçon, 2012), en négligeant de fait tout l'intérêt de cette spécificité française en termes de légitimité et d'intégrité scientifiques qui se comprend dans son organisation générale.

Il nous est donc apparu souhaitable d'analyser les nouvelles dispositions proposées pour mieux en questionner leur portée et leurs incidences, notamment sur le parcours professionnel des enseignants-chercheurs, mais aussi sur le fonctionnement des instances et jurys universitaires (avec le Conseil académique, noté Cac par la suite, et les Comités de sélection, noté CoS par la suite). À cet effet, le chapitre s'organise en trois parties. La première partie présente les dispositifs et modalités antérieurs. La deuxième partie expose les nouveautés introduites par la LPR, et tout particulièrement son article 5 . Enfin, la troisième partie intègre une discussion sur les scénarios qui se dessinent, en exposant les impacts et implications de cette réforme quant à l'articulation des missions du CNU et des établissements universitaires.

\section{L'accès au corps des enseignants-chercheurs jusqu'à la LPR : une démarche nationale au service du local}

Jusqu'à la LPR, le recrutement des enseignants-chercheurs s'appuyait sur une complémentarité entre le CNU, d'une part, considéré comme un acteur clé, garant du statut national des enseignants-chercheurs mais aussi de l'indépendance de la démarche au niveau national, et les instances et jurys d'établissement (CAc et CoS), d'autre part, avec un rôle de recruteur local, en demande d'un tri préalable des candidats compte tenu de la grande diversité scientifique de nombre d'universités.

\section{Les missions du CNU avant la LPR}

Le CNU est une instance nationale composée d'enseignants-chercheurs élus et nommés. Elle est chargée, de manière décisionnaire ou consultative, de nombreuses 
questions ayant trait à la carrière des enseignants-chercheurs : qualification, promotion, PEDR, CRCT, suivi de carrière. Elle s'occupe ainsi de "qualifier " les jeunes docteurs au cours d'une procédure nationale. Un docteur souhaitant postuler à des postes de maître de conférences doit d'abord être qualifié par la section du CNU de sa discipline (à l'exception de candidats en poste à l'étranger, qui peuvent demander une dérogation à la qualification accordée par le $\mathrm{CAc}$ ). Cette procédure de qualification est également déployée pour les maîtres de conférences HDR, qui veulent postuler à des postes de professeurs des universités : ils doivent d'abord être préalablement qualifiés par le CNU (voie 46.1).

Le CNU est composé de 57 sections, chacune correspondant à une discipline. Chaque section comprend deux collèges où siègent en nombre égal d'une part, des représentants des professeurs des universités, d'autre part, des représentants des maîtres de conférences. Comme le note Sonnette (2020)

le CNU était l'instance qui garantissait que les recrutements ne se fassent pas uniquement sur le mode du mandarinat, avec quelques collègues très bien installés dans leur fonction qui ne recrutent que leurs étudiants ou leurs proches, laissant peu de chance à des chercheurs plus isolés, avec moins de connexions ".

Ainsi, au-delà des critiques exprimées, rappelons qu'à sa création, le CNU, en tant qu'organe de suivi de carrière des enseignants-chercheurs par des pairs, se fonde sur les principes de liberté de l'enseignement supérieur et d'autonomie de la recherche, associés à un statut spécifique des enseignants-chercheurs (garantie de protection de l'emploi) pour assurer leur indépendance. Parallèlement, et nonobstant leur statut, la carrière des enseignants-chercheurs est également gérée au sein des établissements.

\section{Au sein des établissements : le CAc et les CoS}

Depuis la loi du 22 juillet 2013 relative à l'enseignement supérieur et à la recherche, chaque université française est dotée d'un CAc. Ce CAc est un organe constitué par la réunion de la Commission de la recherche (CR) et de la Commission de la formation et de la vie universitaire (CFVU) :

- En formation plénière, le CAc est consulté et émet des avis dans de nombreux domaines : " Les orientations des politiques de formation, de recherche, de diffusion de la culture scientifique et technique, la qualification à donner aux emplois d'enseignant-chercheur et de chercheurs vacants ou demandés, la demande d'accréditation, le contrat d'établissement (art.L712-6-1), ainsi que sur toutes les mesures visant à garantir l'exercice des libertés universitaires et des libertés syndicales et politiques des étudiants ".

- En formation restreinte, le CAc est compétent et a vocation à régler les questions individuelles relatives au recrutement, à l'affectation et à la carrière des enseignants-chercheurs. Le CAc constitue en son sein la section disciplinaire. Toutefois, les décisions du CAc comportant une incidence financière sont soumises à l'approbation du Conseil d'administration de l'établissement. À ce jour, les missions relatives à la qualification des enseignants-chercheurs ne représentent qu'une activité mineure des attributions du CAc. 
Au niveau local, un autre dispositif intervient : les comités de sélection (CoS) ont remplacé les commissions de spécialistes depuis 2008. Ils assurent le recrutement des enseignants-chercheurs. Chaque CoS est composé d'enseignants-chercheurs, de chercheurs et de personnels assimilés et de membres extérieurs selon un principe de parité et de double quorum (encadré 1). Reste que dans le rapport de concertation demandé par Madame la ministre Frédérique Vidal sur le recrutement des enseignants-chercheurs, il est précisé que le fonctionnement de ces CoS peut rencontrer des difficultés à respecter les règles de parité (femmes / hommes et personnels internes / externes). Avant la LPR, il existait donc une complémentarité entre les niveaux national et local, certainement à améliorer mais pour autant intéressante, car elle permettait des espaces d'action et d'appropriation par les établissements : traitement différencié par l'établissement des PEDR; partage des promotions entre le CNU et les établissements; prérogative de qualification du CNU et prérogative du recrutement pour les établissements.

\section{Encadré 1 : Les principes de fonctionnement des $\operatorname{CoS}$}

L'article 9 du décret n $84-431$ du 6 juin 1984 sur le statut des enseignants-chercheurs précise qu'un comité de sélection (CoS) est constitué pour pourvoir chaque emploi d'enseignant-chercheur créé ou déclaré vacant dans les établissements publics à caractère scientifique, culturel et professionnel, et dans les autres établissements publics relevant du ministre chargé de l'enseignement supérieur dans lesquels sont affectés des enseignantschercheurs. Le CoS doit être composé à parité de maîtres de conférences ou assimilés et de professeurs des universités ou assimilés lorsqu'il s'agit du recrutement d'un maître de conférences. Pour le recrutement d'un professeur des universités, seuls les professeurs des universités ou assimilés peuvent siéger dans le CoS, en application du principe selon lequel seuls les enseignants-chercheurs d'un rang au moins égal à l'emploi à pourvoir peuvent siéger. Cette règle doit toutefois être combinée avec l'obligation de faire figurer dans les CoS la moitié au moins de membres extérieurs à l'établissement. Par ailleurs, les dispositions de l'article 9-2 du même décret précisent que le CoS siège valablement si la moitié de ses membres sont présents à la séance, parmi lesquels la moitié au moins de membres extérieurs à l'établissement (règle du double quorum).

\section{Un équilibre entre $\mathrm{CNU}$ et établissements}

CNU et établissements coparticipent à la charge du recrutement des enseignants-chercheurs. Un équilibre s'est constitué. L'instance nationale présente de nombreux avantages, pas forcément absolus mais au moins relatifs par rapport aux établissements. D'une part, c'est une évaluation par les pairs scientifiquement ancrée puisque les sections du CNU sont constituées d'enseignants-chercheurs par champ disciplinaire. D'autre part, le CNU offre une évaluation externe à l'établissement qui met à distance les contingences nées d'une gouvernance universitaire à la fois scientifique, économique, mais aussi politique. Ainsi, l'évaluation externe crée une ressource rare, notamment dans les filières qui peuvent connaître des aléas managériaux; elle crée du consensus entre les candidats et agit en tant que juge de paix. Notons que l'instance nationale n'exclut pas l'échelon local : à la qualification aux postes de maîtres de conférences ou de professeurs des universités devant le CNU, s'ajoute l'étape essentielle du recrutement par le CoS et de validation des 
classements des CoS par le CAc restreint et le Conseil d'administration restreint où les représentants de l'établissement ont alors le dernier mot.

\section{LPR : vers un renforcement des établissements au détriment du CNU?}

Avec son article 5 sur la qualification et l'accès au corps des maîtres de conférences (et les articles corollaires), la LPR vient bouleverser les pratiques de recrutement des enseignants-chercheurs puisque, désormais, il est possible de recruter des enseignants-chercheurs sans passer par la qualification du CNU. Elle supprime également l'obligation de qualification par le CNU des maîtres de conférences titulaires qui veulent accéder au corps de professeur des universités. Les établissements auront donc la possibilité de recruter des personnes non qualifiées par le CNU.

\section{La dérogation à la qualification par le CNU}

L'abandon de la qualification par le CNU est présenté par le Législateur dans l'article L. 952-6-3 :

Par dérogation aux articles L. 52-6 et L. 952-6-1 et à titre expérimental, pour les postes publiés au plus tard le 30 septembre 2024, les établissements publics d'enseignement supérieur peuvent demander, après approbation du Conseil d'administration, à être autorisés à déroger pour un ou plusieurs postes à la nécessité d'une qualification des candidats reconnue par l'instance nationale afin d'élargir les viviers des candidats potentiels et de fluidifier l'accès aux corps, cela dans toutes les disciplines à l'exception des disciplines de santé et de celles permettant l'accès au corps des professeurs des universités par la voie des concours nationaux de l'agrégation [...].

Toutefois, le Législateur prévoit un encadrement de la procédure. Ainsi, l'expérimentation est présentée comme une exception qui a pour principale conséquence d'alourdir la charge des CoS en amenant ses membres à se substituer au CNU pour valider les candidats non qualifiés :

Dans ce cas, préalablement à l'examen des candidatures, le comité de sélection, ou l'instance équivalente prévue par les statuts de l'établissement, examine les titres et travaux des personnes qui ne disposent pas d'une qualification reconnue par l'instance nationale, sur la base du rapport de deux spécialistes de la discipline concernée de niveau au moins équivalent à celui de l'emploi à pourvoir. En cas d'avis favorable du comité de sélection, il ajoute les dossiers ainsi qualifiés à ceux des candidats disposant d'une qualification reconnue par l'instance nationale et à ceux des personnes dont la qualification reconnue par une instance nationale n'est pas requise. II procède ensuite à l'examen de l'ensemble de ces candidatures.

Pour souligner le caractère exceptionnel du dispositif, la loi prévoit qu'un rapport évaluera l'expérimentation : “ Au plus tard le $1^{\text {er }}$ janvier 2025, un rapport d'évaluation de l'expérimentation établi par le Haut conseil de l'évaluation de la recherche et de l'enseignement supérieur est remis au ministre chargé de l'enseignement supérieur et transmis au Parlement. Cette évaluation porte notamment sur l'incidence de la dispense de qualification reconnue par l'instance nationale sur la qualité 
et la transparence des procédures de recrutement ». Enfin, le Législateur confie à l'instance la plus haute du droit public le soin de valider les conditions de l'expérimentation déterminées avec les organisations syndicales :

Un décret en Conseil d'État fixe les conditions d'application des dispositions du présent article, après concertation avec l'ensemble des parties prenantes, notamment les organisations représentatives des personnels, les conférences d'établissements et l'instance nationale.

\section{Le lancement $d^{\prime}$ une expérimentation inspirée du modèle américain}

L'expérimentation qui consiste en la possibilité de déroger à la qualification pour recruter un maître de conférences est menée jusqu'en septembre 2024. Toutes les disciplines de santé et celles permettant l'accès au corps des professeurs des universités par la voie des concours nationaux de l'agrégation ne sont pas concernées. La section 06 du CNU étant une section contingentée à agrégation, elle est également exonérée de l'expérimentation sur la qualification des maîtres de conférences. Cette proposition, présentée comme une nouvelle voie de recrutement, est associée à des dispositifs et démarches fortement inspirés du modèle américain (encadré 2), avec la création de chaires de professeur junior et l'instauration de contrat à durée indéterminée de mission scientifique.

\section{Encadré 2 : Les articles supplétifs de la loi, aux modalités à définir}

Une nouvelle voie de recrutement, des contrats de pré-titularisation, pour les directeurs de recherche et les professeurs des universités est instituée : les chaires de " professeurs juniors " (sur le modèle des tenure tracks américaines). Ces derniers bénéficieront d'un financement de 200000 euros en moyenne pour conduire leurs recherches avant de pouvoir intégrer, à l'issue de leurs travaux, les corps des professeurs des universités ou des Directeurs de Recherche. À propos de cette nouvelle voie de recrutement, le Conseil constitutionnel a émis une réserve d'interprétation en rappelant que le principe $d^{\prime}$ 'indépendance des enseignants-chercheurs s'oppose à ce que le chef d'établissement puisse refuser de proposer la titularisation d'un candidat ayant reçu un avis favorable de la commission de titularisation. Un nouvel article 3 bis, issu d'un sous-amendement du Sénat, permet jusqu'en 2024 de déroger à la qualification des maîtres de conférences par le CNU. Un décret doit préciser les conditions de cette expérimentation, " après concertation avec l'ensemble des parties prenantes ». Des contrats postdoctoraux privés et publics sont mis en place afin de faciliter la transition professionnelle des docteurs vers des postes pérennes de la recherche publique ou privée. Un CDI de mission scientifique est créé pour permettre aux établissements publics de recherche et d'enseignement supérieur de recruter des personnels pour mener à bien des projets ou opérations de recherche. Ce nouveau CDI de droit public s'achève avec la réalisation du projet ou de l'opération, ne pouvant être inférieur à une durée de trois ans et supérieur à une durée de six ans. Les parlementaires ont sécurisé la fin du contrat, qui ne peut être rompu (sauf exceptions) la première année.

En prolongement, l'idée est de faire évoluer les voies d'accès au corps de professeurs des universités en développant, à côté de très nombreuses voies existantes (agrégation, voies multiples permises par les articles 46 et s. du décret de 1984 relatif au statut des enseignants-chercheurs), de nouvelles voies pour prendre en la diversité des situations professionnelles et disciplinaires. La préoccupation est de renforcer la 
compétitivité des postes d'enseignants-chercheurs dans le secteur public français : équivalentes à la tenure track, les chaires junior permettent de proposer un parcours accéléré à un jeune chercheur prometteur pour accéder au grade de professeur des universités dans un délai de six ans maximum. À côté de ces nouveaux dispositifs, suite à une réflexion engagée courant 2021 sur les modalités de promotion interne des établissements et sur les critères à adopter pour assurer le passage, hors concours, du corps des maîtres de conférences à celui des professeurs des universités, le repyramidage a été acté par le décret du 20 décembre 2021. Ce dernier crée une voie temporaire d'accès au corps des professeurs des universités et aux corps assimilés. Une liste de 800 promotions, toutes disciplines confondues, a été publiée pour 2021 et 2022 (400 chaque année), à destination de l'ensemble des établissements publics d'enseignement supérieur.

\section{Quelques scénarios : risques, pertes et profits de I'application de la LPR}

La LPR a été adoptée dans un climat tendu avec de nombreuses organisations. Quels que soient les motifs d'opposition, la LPR a constitué une occasion ratée d'échange et de lien entre l'État et une partie importante de son appareil scientifique et d'enseignement supérieur. Pourtant, les constats sur un décrochage de la recherche française se multiplient et présentent même un rare consensus. Les rémunérations dans l'enseignement supérieur et la recherche sont faibles et peinent à attirer dans une concurrence internationale. Ainsi, les derniers prix Nobel français ont en fait réalisé leur carrière à l'étranger : Gérard Mourrou (Physique, 2018) et Esther Duflo (Sciences Économiques, 2019) aux États-Unis, Emmanuelle Charpentier (Chimie, 2020) en Allemagne. La crise de la Covid-19 a encore illustré une telle réalité : la France est le seul membre permanent du conseil de sécurité de l'ONU à n'avoir pas découvert de vaccin. La question demeure de la contribution de la LPR à l'amélioration de la situation de l'enseignement supérieur et la recherche français. Nous proposons ici trois scénarios. Le premier scénario est pessimiste, considérant que la nouvelle loi accentuera les difficultés. Le deuxième scénario est médian : avec de faibles moyens nouveaux, la loi n'aura pas un impact significatif sur la situation. Le troisième scénario est optimiste : la LPR constitue un pas qui va améliorer la situation.

\section{Scénario 1 : la LPR accentue les difficultés}

La LPR pose une ambition. Elle prévoit de nouveaux dispositifs appelés de leurs vœux par certains établissements qui se positionnent comme " intensifs en recherche ". La LPR peut ainsi participer à accentuer le risque d'un enseignement supérieur et de la recherche à deux vitesses : un nombre réduit d'établissements, nourrissant une relation intense avec les organismes de recherche (CNRS, INSERM, etc.), très centrés sur la recherche, d'une part ; un ensemble plus large d'établissements, plus portés sur la formation vécue comme une mission déclassée, qui présentent au mieux quelques fortes spécialisations scientifiques, d'autre part. En soi, cette distinction fondée sur l'excellence ne peut poser de difficultés : la performance scientifique fait le tri entre les chercheurs et entre les établissements. Toutefois, il demeure un postulat étrange. 
II semble que la loi espère faire plus de recherche - mieux même - avec moins de chercheurs et/ou des chercheurs plus précarisés.

En effet, une part importante de la recherche française se fait dans les universités. $Y$ réduire l'effort et les attentes scientifiques ne pourra guère être compensé par un effort dans les établissements intensifs en recherche. Sans compter que c'est faire l'impasse sur la mission de formation qui n'est pas exempte elle-même de contraintes fortes et d'attentes déjà démesurées de la part de l'État y voyant souvent une solution de fortune pour cacher le chômage des jeunes autant que son sous-investissement, là aussi chronique. On peut prendre le problème par tous les bouts, il y a au moins une évidence : pour espérer plus, il faudra investir plus. Dans le même ordre d'idée, la LPR est une loi centrée sur l'activité de recherche dans une acception somme toute étroite, qui omet l'écosystème et le continuum formation-recherche. Les grandes universités internationales présentent à la fois des conditions de recherche attrayantes mais également des formations d'excellence sur des campus aux infrastructures de grande qualité. La recherche repose aussi sur un environnement avec des équipements, des personnels de soutien, une organisation flexible, des conditions de travail attrayantes et une filière qui cherche à former les chercheurs et qui déverse à travers la formation ses résultats dans le champ socio-économique.

\section{Scénario 2 : la LPR a peu d'impact}

Le scénario médian prévoit un impact limité de la LPR. Deux raisons peuvent être avancées. La première raison est d'ordre financier. La loi consent un effort financier en faveur de la recherche, tant les indicateurs viraient au rouge avec à la clé un décrochage de la recherche en France, loin d'un objectif de $3 \%$ du PIB investi dans la R\&D (1 \% public + $2 \%$ privé), avec une réalité à $2,2 \%(0,7 \%$ et $1,5 \%$ respectivement) ${ }^{1}$. Avec la LPR, l'effort pourrait s'approcher des 0,9\% pour la part publique des dépenses en R\&D. La communication gouvernementale a été forte pour annoncer un effort financier qui se révèle somme toute modeste, et qui ne rattrape qu'une faible part du retard de financement de la recherche accumulé en France depuis plusieurs décennies (Larousserie, 2020). Ainsi, les crédits promis s'étalent sur une période particulièrement longue de dix ans, contre sept en général, avec les plus fortes sommes dans les dernières années, ce qui revient à faire des promesses que d'autres seront tenus de respecter.

La seconde raison est d'ordre juridique. Un risque est de créer... des risques notamment juridiques. La LPR prévoit une expérimentation porteuse de risques pour les établissements dans leur recrutement. Si le CNU connaît parfois des difficultés (des sections ont, dans le passé, connu des biais importants favorisant telle ou telle démarche scientifique ou école de pensée), il présente l'avantage d'être une instance collective, nationale, externe aux établissements, qui évalue les candidats à la qualification ou le parcours des enseignants-chercheurs confirmés en produisant un avis objectif, utile à la paix dans les établissements. Les motivations de la loi ne permettent pas d'entrevoir une amélioration du processus de recrutement ou d'évaluation. Au-delà, la LPR méconnaît d'importants pans de la réalité

1 État de l'enseignement supérieur, de la recherche et de l'innovation en France, $\mathrm{n}^{\circ} 14$, Recherche \& Innovation, MESRI-DGESIP/DGRI-SIES. 
scientifique. Ainsi, la recherche est un écosystème. La rémunération des enseignants-chercheurs est une dimension, mais ce n'est pas la seule. La LPR apparaît alors comme particulièrement modeste à la fois dans son ambition financière et dans sa capacité à régler les nombreux problèmes qui frappent l'enseignement supérieur et de la recherche français.

\section{Scénario 3 : la LPR améliore la situation}

Le scénario est ici optimiste. La LPR enclenche une réelle amélioration financière de la recherche. Les enseignants-chercheurs et les chercheurs sont mieux rémunérés avec une attractivité renouvelée de la profession. Les financements sur projets par I'ANR sont plus généreux et plus fréquents, permettant à la fois d'éviter un émiettement des financements qui ne justifient pas le coût concédé à monter les projets et un découragement d'équipes scientifiques face à un taux de réussite excessivement bas par rapport aux standards internationaux (certaines années, inférieur à $10 \%$, quand la norme internationale est de 25 à $30 \%$, notamment pour éviter le découragement). Le recrutement des personnels est plus ouvert, notamment en direction de chercheurs internationaux à haut potentiel pour lesquels le processus de recrutement reposant sur une qualification par le CNU et la sélection par un CoS s'avérait aussi lourd que lent, malgré des dérogations possibles.

Ce scénario souffre toutefois d'une limite : la LPR, on l'a vu, constitue in fine un effort modeste qui ne rattrape pas le retard accumulé, et qui ne concerne que la recherche dans une acception étroite alors qu'au même moment, les principaux pays développés accentuent leurs efforts scientifique et technologique. Ainsi, la LPR ne constitue au mieux qu'un début d'effort financier. Celui-ci devra être, bien plus que maintenu : amplifié. Il devra également s'adosser à une refonte organisationnelle et institutionnelle avec, éventuellement, la consultation de la communauté scientifique. Parmi les mesures complémentaires, dans le chantier RH posé par la LPR, deux directions semblent importantes. La première direction consiste à formuler une complémentarité entre le CNU et les établissements : l'enjeu de la GRH dans l'enseignement supérieur et la recherche réside dans une meilleure articulation des missions et d'un étroit partenariat entre les instances du niveau national et local. Le défi est donc de mobiliser les atouts des instances clés de la GRH des enseignants-chercheurs - sans les opposer ou les exclure - pour créer un dispositif performant permettant à la fois une approche globale et disciplinaire des enseignants-chercheurs, capitale en terme d'intégrité scientifique, et une vision territoriale adaptée aux réalités des établissements, dorénavant autonomes, dans les trois métiers que sont la recherche, l'enseignement et la participation à la gouvernance des établissements.

La seconde direction consiste à construire une véritable fonction $\mathrm{RH}$ dans les établissements. Ceci implique notamment un renforcement des moyens disponibles pour assurer, non un contrôle mais une gestion qui couple spécificités scientifiques et disciplinaires et mise en place de pratiques opérationnelles adaptées, notamment en matière de recrutement et d'accompagnement dans le long terme. Les défis à relever en la matière sont importants tant les limites des pratiques de recrutement actuelles sont connues : le temps contraint consacré au recrutement par les CoS (des auditions d'une durée de 20 à $40 \mathrm{mn}$ ) ; le manque de clarté des critères de sélection, avec l'impératif de recrutement pour ne pas perdre le poste; le décalage du 
recrutement centré sur la recherche avec les réalités de l'exercice, parfois très chargé par l'enseignement ; l'absence de suivi des recrutements ; la question des mutations (point d'équilibre à trouver entre le droit à la mutation, donnée fondamentale du statut de fonctionnaire d'État, et l'autonomie de recrutement). II s'agit alors de développer une transparence et une rigueur dans la procédure de recrutement par une définition rigoureuse du profil du poste, une présence significative d'extérieurs, mais aussi de recruteurs.

Ainsi, le rapport demandé par le ministère de l'Enseignement supérieur, de la Recherche et de I'Innovation sur le recrutement des enseignants-chercheurs à Fabienne Blaise, Pierre Desbiolles et Patrick Gilly propose que soit adjoint au jury un "référent recrutement ", représentant de l'établissement. Ce référent participerait à plusieurs recrutements. II apporterait ainsi un regard transversal et neutre sur l'ensemble des recrutements de la composante ou de l'établissement. II s'agit également d'accompagner l'intégration des enseignants-chercheurs recrutés, pour renforcer le sentiment d'appartenance et l'adaptation aux particularités organisationnelles et territoriales de l'établissement. Paradoxalement, ce même rapport propose de ne plus intégrer de membres extérieurs dans les CoS en limitant leur participation à un rapport écrit sur les candidats, sans présence lors des débats. On voit ici que l'alternative au CNU se cherche encore.

\section{Conclusion}

Au final, les questionnements du chapitre illustrent les enjeux actuels de la gestion publique des ressources humaines, en étant à la croisée d'une approche historique, de nature administrative, qui montre ses limites, et d'une approche renouvelée, qui doit s'ancrer dans les spécificités du service public de la recherche et de l'enseignement, dont le statut d'enseignant-chercheur est l'un des fondements majeurs. C'est donc bien un défi d'innovation singulier et ad hoc qui est attendu par l'ensemble de la communauté scientifique et universitaire. La tentation qui consisterait à ne pas reconnaître les spécificités du monde de la recherche et de l'enseignement supérieur, et à ne pas partir de l'existant, serait une erreur majeure pour l'avenir. II convient ainsi de développer une organisation pérenne de la recherche, qui garantisse aux futures générations de chercheurs la perspective d'un emploi stable, propice à une recherche sereine, autonome et collaborative, et de préserver l'attachement au service public de la recherche et de l'enseignement comme garantie de la qualité et de l'indépendance de leurs activités scientifiques, mais aussi comme atout de GRH. 


\title{
Technologies et CNU
}

\author{
Une nécessaire synergie
}

Marc BIDAN, Jean-Fabrice LeBRATY, Jean-François Lemoine et Nicolas LeSCA

Débutons le chapitre par une petite fiction. 10 janvier 2023, 9 h 45 : la présidente de la section 06 du CNU reçoit un message sur le fil « Slack » dédié du ministère qui lui annonce que l'étude des dossiers de CRCT peut débuter. Elle lance alors l'application "Match » qui regarde les caractéristiques des dossiers et les met en regard des compétences de rapporteurs. Les contraintes d'éthique sont aussi respectées. Fondé sur une IA en cours d'apprentissage, un choix lui est alors proposé. Elle en discute via Zoom avec son bureau pour affiner les allocations. Certes, il y a une petite heure de travail mais ce n'est pas grave; en 2024, cela ira encore plus vite. À 14 h 45, elle valide les choix définitifs. Ils sont alors automatiquement stockés dans une blockchain publique avec permissions. Les candidats, comme les rapporteurs, peuvent alors se connecter et utiliser leur clé privée pour vérifier leurs rapporteurs et les adresses d'envoi des documents complémentaires.

Ce scénario est une fiction mais, pourtant, il ne comporte que des technologies existantes et utilisées par ailleurs. À titre d'exemple, plus de 12 millions d'utilisateurs disposent de "Slack » et un nombre encore plus grand de l'application Teams qui le concurrence dans la même catégorie : les outils de travail collaboratif. L'application " Match » est un nom inventé pour l'occasion. Mais cette fonctionnalité d'allocation est utilisée, par exemple, par l'éditeur suisse de revues Frontiers afin de proposer à un éditeur d'un numéro spécial des réviseurs adaptés pour les articles soumis. Enfin, les blockchains publiques avec permissions sont nombreuses. Carrefour et Nestlé en ont déployé une pour rassurer leurs clients sur l'origine d'un de leurs produits les plus sensibles : le lait bio infantile.

Le but du présent chapitre est de proposer un panorama des technologies existantes et qui pourraient être utiles pour, premièrement, accompagner les membres du CNU dans leurs différentes activités et, deuxièmement, permettre la mise en œuvre de nouvelles missions. Ainsi, la contribution évoquera à la fois comment les technologies mises en œuvre au niveau du CNU peuvent se positionner 
en soutien des membres de la communauté, sans pour autant éluder les risques émergeant de ces nouveaux usages.

\section{Les technologies numériques et le CNU : le traditionnel arbitrage entre opportunités et risques}

Traditionnellement, le CNU fait l'objet de diverses interrogations relatives à son organisation, à son mode de fonctionnement, à la manière dont les dossiers sont instruits en son sein, et à l'objectivité des décisions rendues. Si la plupart de ces questions sont légitimes, force est de constater qu'elles trouvent généralement toutes leurs origines dans un déficit d'information des candidats vis-à-vis du CNU. Ces derniers s'interrogent en effet fréquemment sur les processus de décision des membres du CNU, et sur leurs critères d'évaluation d'un dossier. Dans le même temps, les données dont disposent les candidats sur le fonctionnement du conseil ne proviennent pas toujours de sources véritablement fiables et relèvent parfois de la pure imagination, de la rumeur, voire d'un certain niveau de phantasme. Dans tous les cas, on constate une très forte asymétrie d'information entre les différents acteurs concernés par le CNU : ceux qui déposent un dossier et ceux qui ont en charge son évaluation.

Avec l'arrivée massive des technologies numériques au cours des dernières années, l'information a davantage circulé et son accès a été considérablement facilité. Est-ce pour autant un avantage systématique pour les candidats ? Ne peut-on pas voir dans la libre circulation des données des risques pour ces derniers et, si oui, lesquels? Par ailleurs, cette réduction de l'asymétrie de l'information, via les technologies numériques, n'impacte-t-elle que les candidats? N'exerce-t-elle pas également une influence sur les membres du conseil et, si oui, laquelle ? C'est à ces différentes interrogations que nous nous proposons de répondre dans la première partie du chapitre en prenant soin de systématiquement distinguer les opportunités et les risques que les technologies numériques représentent tant pour les candidats qui déposent un dossier au CNU que pour les membres du conseil qui ont la responsabilité de l'évaluer.

Technologies numériques et candidats : d'un accès simplifié aux modalités de fonctionnement du CNU à un devoir d'extrême transparence dans la constitution des dossiers

Indéniablement, la dématérialisation complète des dossiers à destination du CNU ${ }^{1}$ présente des avantages pour les enseignants-chercheurs. Elle facilite leur envoi, supprime les coûts financiers importants liés à l'expédition de documents lourds et volumineux (thèses, articles, ouvrages), et laisse plus de temps, en théorie, aux candidats pour les préparer. Dans le même temps, l'accès à l'information relative au

1 Les dossiers adressés au CNU peuvent concerner une demande de qualification pour les maîtres de conférences, de promotion pour les professeurs des universités et les maîtres de conférences, de PEDR pour les professeurs des universités et les maîtres de conférences, de congés pour recherches ou conversion thématique (CRCT) pour les professeurs des universités et les maîtres de conférences. 
fonctionnement du CNU et aux critères d'évaluation des dossiers est devenu direct et aisé. Il suffit, pour y accéder, d'aller consulter sur le site du CNU les rapports que le (la) président(e) rédige systématiquement après chaque session. Ces derniers constituent une source d'information précieuse et objective à destination des candidats. À titre d'exemple, le rapport sur la qualification aux fonctions de maîtres de conférences de la session 2021 renseigne sur le nombre de dossiers reçus, le pourcentage de dossiers qualifiés, le déroulement de la procédure de qualification, les éléments de doctrine du CNU en matière de qualification (évaluation des aptitudes à la recherche, à l'enseignement, à l'implication collective), les pièces à fournir dans le dossier. Grâce à cette mise en ligne de l'information, il est dorénavant possible d'accéder à toutes les données utiles à la constitution d'un dossier, quel que soit l'établissement fréquenté ou l'unité de recherche à laquelle chacun est rattaché. Les technologies numériques peuvent donc être considérées comme un moyen d'assurer l'équité de traitement de chaque enseignant-chercheur souhaitant déposer un dossier au CNU.

Bien entendu, le fait que chaque candidat puisse disposer des données relatives aux critères d'évaluation des dossiers rend naturellement les évaluateurs plus exigeants quant au respect de ces derniers. Avant de se décider à faire une demande au $\mathrm{CNU}$, il est donc recommandé à chaque enseignant-chercheur de mener sa propre auto-évaluation afin de s'assurer de l'adéquation - tant sur la forme que sur le fond - de son dossier aux attentes des membres du CNU. Cette remarque est particulièrement importante pour les doctorants issus de disciplines qui ne relèvent pas directement des sciences de gestion et du management (par exemple, l'économie, l'informatique, la communication), mais qui tentent néanmoins d'être qualifiés dans la section 06. Si la dématérialisation des dossiers leur permet d'adresser sans effort particulier le même dossier à deux (voire plus) sections différentes du CNU, les chances de succès d'une telle opération s'avèrent plus que réduites. On ne peut en effet prétendre répondre aux attentes des membres d'une communauté à l'aide d'un dossier qui n'est pas adapté aux codes et aux attentes de cette dernière, voire qui a été construit selon les us et coutumes d'une autre section. Dans ce cas précis, les technologies numériques ne rendent pas particulièrement service aux candidats.

Si les candidats ont dorénavant un accès simplifié et direct - grâce aux technologies numériques - à des informations importantes pour la constitution de leurs dossiers, une exigence de transparence au niveau des données fournies s'impose à eux de manière encore plus forte qu'auparavant. Plus précisément, toute information communiquée dans un dossier est immédiatement vérifiable par les évaluateurs qui le souhaitent. II convient donc d'éviter de fournir des éléments ambigus, plus ou moins précis ou argumentés, car ils amèneront les rapporteurs à vouloir éclaircir la situation en menant des recherches complémentaires sur Internet. Ainsi, la consultation d'une page LinkedIn d'un enseignant-chercheur peut permettre d'en apprendre plus, et de manière plus claire et précise, que le CV qu'il a pris soin d'envoyer et de remplir de façon plus ou moins exhaustive. Par l'intermédiaire des technologies digitales, il est dorénavant possible d'accéder à des données qui étaient beaucoup moins faciles à trouver dans le passé telles que les activités administratives et/ou collectives véritablement exercées par le candidat, les institutions qu'il a fréquentées, les articles réellement acceptés par les revues, les classements des articles à la date d'acceptation du papier (et non à celle de la constitution du dossier). Le souci d'honnêteté et de transparence doit donc être au rendez-vous lors de la constitution 
d'un dossier puisqu'il est dorénavant possible de tout vérifier. Les technologies numériques ne font pas qu'apporter des avantages aux individus; elles lui imposent également de nouveaux devoirs.

Technologies numériques et membres du CNU : d'un possible contrôle renforcé des dossiers constitués à un accroissement de l'auto-contrôle entre pairs

Pour les membres du CNU, l'évaluation de la véracité des données fournies par les candidats est devenue beaucoup plus simple. La place que pouvait occuper par le passé le doute dans l'appréciation des dossiers s'est considérablement réduite dans la mesure où tout est dorénavant contrôlable en un temps réduit. Comme on l'a souligné, le fait de pouvoir consulter quasi instantanément des informations, issues de sources différentes (page LinkedIn d'un enseignant-chercheur, site Internet de son unité de recherche, de l'école doctorale où ses doctorants sont rattachés, etc.), sur un candidat permet aux rapporteurs du CNU d'avoir, en cas d'incertitude, une vision plutôt précise et exacte sur la réalité de son activité professionnelle. Dans le cadre de l'examen des demandes de PEDR, il arrive parfois que les indications fournies par les candidats soient imprécises, notamment en matière de devenir des docteurs, de nombre de thèses soutenues, de pourcentage d'encadrement pour chaque doctorant. La consultation simultanée de diverses bases de données permet aux rapporteurs d'affiner leurs évaluations, de dissiper leurs doutes éventuels sur une partie d'un dossier à évaluer et, surtout, d'évaluer la véracité des propos tenus. En ce sens, les technologies numériques facilitent le travail assigné aux membres du CNU.

Si les technologies numériques imposent aux candidats un devoir de transparence accru en matière de constitution des dossiers, elles imposent également cette obligation aux rapporteurs en matière d'évaluation des candidats. Du fait de l'accès instantané des membres du CNU, durant les sessions, aux données relatives à l'ensemble des candidats, les rapporteurs se doivent d'être les plus précis et les plus complets possible lorsqu'ils évaluent les enseignants-chercheurs, au risque d'être interpellés directement par les autres membres du CNU en cas de restitution incomplète du parcours professionnel des candidats ou d'oublis importants les concernant. Les technologies numériques renforcent donc l'auto-contrôle par les pairs du travail d'évaluation réalisé par chaque membre du CNU. Tout comme les candidats, les rapporteurs se trouvent ainsi en situation d'évaluation durant les sessions et aucun d'entre eux n'a d'intérêt à voir son image d'évaluateur se dégrader vis-à-vis de ses pairs. Une telle situation doit donc rassurer les enseignants-chercheurs qui expriment souvent des inquiétudes quant à la manière dont on rapporte sur eux au CNU. Avec l'essor des technologies numériques, les risques d'expertise imprécise et incomplète sur un candidat s'avèrent quasi-inexistants tant le pouvoir exercé par les pairs sur les rapporteurs est devenu fort. 


\section{Des technologies au service des activités}

Analyse des dossiers, détection de bons profils ou encore gestion administrative, d'un côté, big data, IA, blockchain, de l'autre. Voyons comment activités et technologies peuvent se marier.

\section{Big data et analyse des candidats}

Le concept de big data, même s'il a plus de dix ans, demeure plus un sujet de fantasme que de réalité entrepreneuriale. En effet, peu d'organisations sont capables de générer, stocker et analyser les données massives. À titre d'exemple, quasiment aucune administration publique ne gère de big data en France et, par exemple, aucune banque privée non plus. Faut-il pour autant abandonner le domaine ? La réponse est bien évidemment négative. Même si le CNU ne gérera pas de big data, il peut utiliser certains des outils employés pour gérer du big data. C'est le cas du logiciel R. R est un logiciel dédié à l'analyse de données. Certes, ce n'est pas nouveau. SPSS fut un temps la référence en la matière. Cependant, $R$ se distingue par sa gratuité, conséquence de son appartenance au mouvement du logiciel libre (rattachement à $\mathrm{GNU}$ ). En outre, $\mathrm{R}$ est à la fois un environnement et un langage de script. Ainsi, profitant de l'engouement lié à la mise à disposition de gros volumes de données, une communauté s'est agrégée autour de $\mathrm{R}$ et met à disposition un nombre croissant de " packages ", c'est-à-dire un script dédié à une fonctionnalité qui permettra d'être appelé pour répondre à un problème plus large. À ce jour, plus de 17500 packages sont disponibles. Parmi eux, une dizaine sont orientés sur l'analyse des publications, et tout particulièrement sur la mesure de la performance d'un chercheur. Examinons certains de ces packages.

RImpact annonce mesurer l'impact académique d'un auteur. II se fonde sur un article, publié en 2012, qui propose une méthode agrégative (22 critères) permettant de noter un auteur (Ruscio et al., 2012). Le package "Scholar » va quant à lui plus loin. En effet, il se fonde sur Google Scholar qui, utilisant la puissance du moteur de recherche de Google, propose une liste des publications de chaque auteur. Précisions qu'année après année, la qualité des données progresse (dédoublonnage ou suppression des homonymes, par exemple). À partir de ces données, le package propose différentes analyses classiques (nombre, classement parmi d'autres auteurs, en tenant compte aussi de l'ordre dans lequel l'auteur est cité). Il est donc possible de classer automatiquement l'ensemble des dossiers de candidats et ce, quel que soit le nombre de candidats. Le package propose aussi des fonctions utiles comme le fait de savoir dans combien de revues différentes une personne a publié. Mais, plus en avant, "Scholar " propose de prédire les futurs $h$ index en fonction des publications passées et de la discipline du candidat (Acuna et al., 2012). "Tinyscholar ", de son côté, propose juste une représentation visuelle des données issues aussi de Google Scholar, ce qui permet de donner une vue d'ensemble d'un chercheur de manière ergonomique.

Pour aller plus dans le détail de la production d'un candidat, le package " Crminer » va rechercher tous les textes disponibles via le Crossref d'un auteur. II est possible alors de lancer tout un ensemble d'analyse sur le contenu ainsi récupéré et d'avoir des indicateurs quant aux thématiques développées par le candidat. Mais aussi, il est 
possible de connaître, par exemple, sa qualité d'écriture au travers, par exemple, de la complexité du vocabulaire qu'il emploie. En effet, les packages permettant d'analyser le langage NLP sont très nombreux et utiles notamment pour la recherche en sciences de gestion et du management (Balech et Benavent, 2019).

Ainsi, le CNU peut profiter des outils de big data ( $R$, par exemple), mais aussi d'entreprises utilisant le big data (Google, notamment) pour analyser les dossiers de publication des candidats. Bien évidemment, ce processus ne va pas sans soulever un certain nombre de questions. Première question : que se passe-t-il si les publications ne sont pas indexées par Google Scholar? Pour lancer le débat, il est aisé de mettre en avant que le service va notamment chercher ses données dans des bases comme HAL. Or, il est plus que recommandé aux chercheurs de mettre, a minima, leurs références dans HAL. Deuxième question : quels critères retenir ? C'est ici que le CNU trouve tout son intérêt. En coordination avec la FNEGE et les associations académiques, des critères globaux peuvent émerger. Ces critères peuvent alors permettre d'aller plus loin que les critères existants. Finalement, il existe deux critères simples : " au moins deux articles dans des revues classées sur quatre ou cinq ans » et le fameux " deux rang 2 ". De tels critères sont l'illustration de l'impossibilité de disposer de données fiables permettant un calcul. Aujourd'hui, ces données commencent à être disponibles. Aussi, et avant que d'autres nous imposent leurs critères, il serait judicieux de les proposer et de les tester.

\section{IA et détection des profils}

Rappelons tout d'abord que le CNU a pour missions principales le recrutement ainsi que le suivi des carrières des maîtres de conférences et des professeurs des universités $^{2}$. Ainsi, toute demande confondue, la section 06 du CNU, par exemple, étudie chaque année près de 2000 dossiers et émet autant d'avis qualitatifs et fondés sur des faits observables dans l'activité des candidats. Ce nombre pourrait encore augmenter significativement si le suivi de carrière, prévu par les articles 7-1 et 18-1 du décret $n^{\circ} 84-431$ du 6 juin 1984 ${ }^{3}$, modifiés par l'article 21 du décret n²014-997 du 2 septembre 2014 et par l'article 2 du décret n²019-1108 du 30 octobre 2019, était mis en œuvre, généralisé et systématisé. Chacune de ces campagnes est traitée selon un processus propre et des critères spécifiques. Néanmoins, tous ces processus ont en commun trois étapes critiques et fondamentales :

- Une première étape d'affectation, au terme de laquelle les dossiers des candidats demandeurs sont répartis aussi équitablement que possible entre l'ensemble des membres du CNU qui siègeront lors de cette session, tout en veillant à éviter les conflits d'intérêts avérés (par exemple, lorsqu'un dossier est évalué par le co-auteur d'un candidat) ou potentiels (par exemple, lorsqu'un dossier est évalué par un rapporteur appartenant à la même université que le candidat).

- Une deuxième étape d'évaluation, au terme de laquelle chaque dossier est étudié en profondeur par deux rapporteurs, selon des critères objectifs communs

2 [https://www.enseignementsup-recherche.gouv.fr/m/cid22711/c.n.u.-et-cnap-les-instances-nationales.html], consulté le 13 juin 2021.

3 [https://www.legifrance.gouv.fr/loda/id/LEGIARTI000039331026/2021-05-16/?isSuggest=true], consulté le 16 avril 2021. 
et partagés entre tous les membres du CNU qui siègeront lors de cette session, pour garantir l'équité et la comparabilité entre les dossiers.

- Une troisième étape de décision collective, au terme de laquelle l'ensemble des membres du CNU qui siègent à la session, sur la base des rapports et des avis motivés et objectivés des rapporteurs, prennent collégialement une décision pour chacune des demandes étudiées.

Tandis que les entreprises publics et privées de moyenne et grande taille se sont déjà largement dotées d'outils de gestion (prévisionnelle) des carrières et des compétences, les sections du CNU, quant à elles, ne peuvent encore compter que sur la capacité d'organisation de leurs bureaux, et sur l'intelligence et la rationalité humaine de chacun des membres. Pourtant, la diversité des contextes institutionnels, la variété des trajectoires professionnelles individuelles et la quantité d'informations qualitatives à prendre en considération font de leur mission d'évaluation et de décision une tâche complexe face à laquelle les rationalités humaines et collectives sont confrontées à leurs limites. Sans chercher l'exhaustivité, mentionnons-en quelques-unes :

- L'affectation des dossiers se fait sur la base des informations connues et visibles sur les candidats et les évaluateurs. Par exemple, leurs liens de parenté apparents, leurs universités d'appartenance, leurs activités de recherche communes, leurs liens d'influence réciproque éventuels, etc. En revanche, tout lien qui n'est pas connu du CNU, ou déclaré par ses membres, reste invisible et ne peut donc pas être pris en compte pour optimiser l'affectation et la garantir exempte de tout biais. Cette étape d'affectation s'apparente ainsi à la résolution d'une équation à variables multiples. En situation d'information imparfaite et distribuée, tendre vers la résolution de cette équation - au gré d'interactions multiples avec les évaluateurs - est un exercice chronophage, certes garant du respect de l'équité, mais sans réelle création de valeur. Ici, une IA de type fouille de textes et de données (text and data mining, TDM), circonscrite aux dossiers des candidats ainsi qu'aux CV extensifs des membres du CNU, permettrait d'automatiser des scénarios d'affection. Une fouille de texte élargie aux sites académiques de référence (par exemple, Research Gate, HAL, etc.) ${ }^{4}$ permettrait d'élargir encore la base de la recherche de traces de conflits d'intérêt possibles et de les prendre en considération dans les scénarios d'affectation. Se poseraient alors les questions de la liste précise et exhaustive des situations de conflit pour programmer I'IA et des sites de référence pertinents à fouiller.

- L'évaluation des dossiers repose autant sur l'information transmise par le candidat - dans sa complétude comme dans sa précision - que sur l'appréciation et la capacité de l'évaluateur à identifier l'information pertinente et à en évaluer la fiabilité. Cette étape d'évaluation s'apparente ainsi à un travail chronophage de recension et de catégorisation d'informations - parfois aussi de complétion lorsque des précisions manquent et de fiabilisation lorsque des contradictions apparaissent dans le dossier - tandis que la réelle valeur ajoutée de l'évaluation

4 La directive UE2019/790 du 17 avril 2019 sur le droit d'auteur et les droits voisins dans le marché unique numérique prévoit la transposition des exceptions de fouille de texte et de données (TDM). Le gouvernement français doit encore transposer par ordonnance les dispositions du texte européen. 
repose avant tout sur une appréciation qualitative d'ensemble du potentiel ou de la trajectoire d'un candidat. Ici aussi, une IA de type TDM multilingue, qui procèderait, sur la base d'une grille d'analyse précise et détaillée, à un ordonnancement et à une classification des faits observables de l'activité professionnelle, permettrait d'automatiser un pré-rapport formaté par candidat. Les rapporteurs pourraient alors concentrer leur attention sur l'évaluation de l'activité et de la trajectoire des candidats qui leur sont affectés. Une IA de type apprentissage profond (deep learning) pourrait également apprendre des corrections qu'apporteraient les rapporteurs sur leurs pré-rapports pour aider le système à reclasser des informations et à en détecter d'autres. Une question fondamentale se poserait alors : faut-il s'en remettre exclusivement aux informations déclarées dans le dossier du candidat (fouille circonscrite) ou permettre à I'IA de chercher au-delà de l'information déclarée (fouille élargie) ?

Enfin, comme tout collectif élu évoluant tous les quatre ans, l'existence d'une mémoire organisationnelle se pose aussi : mémoire des processus, des candidats, des dossiers, des décisions, et des contextes historiques et institutionnels de notre métier pour encore mieux évaluer les dossiers. Ici, une IA apprenant sessions après sessions, en utilisant, par exemple, des algorithmes bayésiens, contribuerait à faire émerger une mémoire organisationnelle. Une telle mémoire serait d'autant plus importante et pressante que le CNU serait appelé de ses vœux par son ministère de tutelle à s'engager plus fortement dans le suivi de carrière des enseignants-chercheurs.

\section{Blockchain et smartcontracts}

La contribution possible de I'IA aux processus d'affectation-évaluation-décision des dossiers maintenant éclairée, se pose la question de la circulation des dossiers entre les candidats et les membres du CNU. Quelles sont les tendances technologiques émergentes qui pourraient contribuer à soutenir ce processus de circulation ? Nous pensons bien évidement à la blockchain et aux smartcontracts. La blockchain repose sur trois fondements théoriques et technologiques bien connus : la théorie des jeux, le réseau de pairs à pairs et la cryptographie asymétrique. Elle consiste en un registre distribué qui stocke de manière permanente et inaltérable un ensemble de transactions (par exemple, des crypto-monnaies ou des jetons), validées par un consensus (par exemple, la preuve de travail et la preuve d'enjeu). On distingue notamment deux types de blockchain selon qu'elle est publique ou privée :

- dans une blockchain privée, seuls les membres ou utilisateurs autorisés peuvent accéder au registre distribué, la nature, le statut ou l'identité des participants à la blockchain étant déterminés dès l'origine ;

- la blockchain publique est conçue pour que tout le monde soit en mesure d’accéder au registre distribué, moyennant éventuellement de s'authentifier.

Dans le contexte du CNU, un dossier pourrait ainsi être représenté par un jeton sur une blockchain. Il existe des jetons fongibles qui représentent des objets interchangeables (par exemple, un billet de cinq euros ou des crypto-monnaies) et des jetons non fongibles qui permettent de représenter des objets individualisables (par exemple, un dossier $\mathrm{CNU}$ ). Lorsque les jetons sont déployés sur une blockchain, ils en prennent alors les caractéristiques : décentralisation, immutabilité, confidentialité, intégrité, 
authenticité, non répudiation, auditabilité. Pour faire circuler les jetons entre les participants à une blockchain (par exemple, les membres du CNU, mais aussi les candidats qui souhaitent soumettre leur dossier au CNU), il est nécessaire qu'ils détiennent chacun un portefeuille électronique, ou Wallet, avec un numéro de compte. La circulation est alors opérée par un programme informatique appelé smartcontract.

Contrairement à son nom, un smartcontract n'est pas un contrat au sens juridique et il n'est pas intelligent non plus. II s'agit en fait d'un code informatique enregistré dans le registre d'une blockchain qui fournit un environnement d'exécution ou un langage de script. L'exécution d'un smartcontract est déclenchée par une transaction et son résultat est soumis au consensus. Les smartcontracts peuvent être implémentés sur toutes les blockchains privées ou publiques qui supportent les programmations de smartcontract. Par exemple, la blockchain Ethereum pourrait être mobilisée pour faire circuler les dossiers des candidats représentés par des jetons non fongibles grâce à des smartcontracts. On peut alors programmer un smartcontract pour faire circuler un jeton et donc programmer autant de smartcontract qu'il y a de dossier à faire circuler. Mais on peut aussi programmer un smartcontract générique et l'appeler chaque fois que l'on a besoin de faire circuler un dossier. Les membres du CNU recevraient alors l'ensemble des jetons dans leur Wallet.

Il faudrait ensuite faire le rapprochement entre l'empreinte numérique d'un jeton (dans leur Wallet) et le dossier physique d'un candidat (par exemple, sur Galaxie) pour pouvoir commencer à l'étudier. Automatiser ce rapprochement impliquerait toutefois le développement d'une application spécifique qui permettrait d'abord de lier un jeton au dossier source du candidat, puis de télécharger ou de lire le dossier en question. Cela impliquerait donc l'existence d'un fichier centralisé, hors de la chaine, qui rassemble la concordance entre le dossier source et le jeton déployé sur la blockchain. Cela nécessiterait aussi l'existence d'une plateforme de partage de documents (par exemple, Galaxie) dans laquelle seraient stockés les dossiers sources des candidats. L'existence à la fois d'un fichier et d'une plateforme de partage de documents - tous deux centralisés - serait finalement en contradiction avec la philosophie de la blockchain, dont l'objectif est de recourir à un réseau de pairs à pairs distribués pour éviter de dépendre d'une infrastructure centralisée. Appliqué au contexte de la circulation des dossiers du CNU, l'intérêt d'adopter une blockchain prête donc à discussion :

- La blockchain est pertinente lorsqu'il s'agit de faire circuler de la valeur entre un nombre important de parties prenantes qui ne se connaissent pas et ne se font pas confiance. Dans ce cas, le recours à un réseau de pairs à pairs distribués peut être une solution pour renforcer la confiance. Mais un tel enjeu de confiance entre les candidats et les membres du CNU est-il tellement important qu'il justifierait le recours à la technologie blockchain?

- La blockchain est pertinente quand la transaction ou le jeton ont besoin d'être fiabilisés. Le protocole blockchain et le chiffrement asymétrique des dossiers assurent alors une circulation sécurisée entre les parties prenantes et diminuent le risque d'attaque information et de corruption des transactions. Mais les niveaux de vulnérabilité des plateformes du CNU et les niveaux de sensibilité des sujets traités par le CNU (qualifications, promotions, suivi de carrière, etc.) sont-ils tellement élevés qu'ils justifieraient le recours à la technologie blockchain ? 
- Le bilan énergétique de la blockchain est également considérablement plus élevé que celui d'une plateforme centralisée. Le recours à un smartcontract générique serait, certes, une solution moins énergivore que le déploiement systématique pour chaque transaction, mais son impact environnemental resterait néanmoins considérable comparativement à l'utilisation d'une plateforme de partage de documents. L'intérêt pour la technologie blockchain doit certes être considéré au regard du processus de circulation de transaction à soutenir mais aussi de la frugalité énergétique recherchée.

Ainsi, l'intérêt d'adopter la technologie blockchain pour organiser, sécuriser et fiabiliser la circulation des dossiers du CNU questionne. Néanmoins, le déploiement de smartcontracts, même hors d'une blockchain, semble tout à fait pertinent en tant que code informatique qui permet de faire circuler de l'information entre tiers.

\section{IoT et gestion administrative des membres du CNU}

Le concept d'Internet des Objet (loT en anglais, et en acronyme en général) a une vingtaine d'années mais ne se matérialise concrètement que depuis une dizaine d'années. L'idée globale est d'avoir des appareils connectés ayant peu de capacités mais remplaçant ce manque par leur nombre et leur interconnectivité. Le concept est d'ailleurs plus ancien. K. Kelly, ancien éditeur de Wired et générateur de nombreuses idées dans les mondes numériques, indiquait dès 1998 que la première règle pour la nouvelle économie se fondait sur le volume des connexions (Kelly, 1998). Il estimait ainsi déjà que de nombreux objets simples connectés étaient plus puissants qu'un gros " cerveau ». Les objets connectés prennent différentes formes et souvent captent des données issues de leur environnement (chaleur, humidité, présence etc.). Le marché de l'loT est prometteur. En effet, si le nombre d'ordinateurs se compte en centaines de millions, celui des smartphones en milliards, on estime qu'il y aura plus de 30 milliards d'objets connectés dès 2025. Concernant la connexion, des entreprises comme SigFox ont contribué à la réalisation d'infrastructures de communications dédiées. Notons d'ailleurs qu'en quelques années, SigFox a bâti un réseau de communication couvrant l'Europe notamment, ce qui, en comparaison des opérateurs de téléphonie, est impressionnant. De plus, certains objets peuvent être intégrés à des smartphones ou à des ordinateurs (Raspberry ou Arduino, par exemple).

Comment, le CNU pourrait-il profiter de ces objets ? Plusieurs pistes peuvent être identifiées. Première piste : quand en 2021, il est encore demandé de signer une feuille de présence papier comme preuve de présence, cela est plus que désolant. La perception d'un simple objet connecté identifiant son porteur et passant près d'une borne installée dans la salle permettrait à la fois de s'assurer de la présence et aussi de respecter les consignes sanitaires limitant la transmission de virus. Pour les sceptiques, il n'y a pas plus de risques d'usurpation d'identité avec un objet qu'avec une signature papier. Nous dirions même moins car cela nécessite que le membre donne ou se fasse dérober son objet pour que l'usurpateur puisse s'identifier. Tandis que personne n'est en mesure de vérifier une signature manuscrite. Seconde piste : le même objet pourrait être utilisé pour le vote. En effet, l'objet pourrait posséder trois boutons (oui, non, blanc), et chaque membre passant devant la borne appuierait de 
manière discrète pour donner son choix. Fini ainsi les petits papiers blancs, le long dépouillement et le PV notant les résultats. D'autres idées peuvent émerger bien sûr. En tous cas, ces petits objets seraient très utiles pour permettre au membre du CNU de ne se concentrer que sur leur travail, et non sur la bureaucratie de papier.

\section{Réseaux sociaux et promotion des disciplines}

La question de la relation entre les réseaux sociaux au sens large - c'est-à-dire que nous évoquons ici avant tout une " application " définie comme une " plateforme d'intermédiation et de mise en contact " - et la promotion des disciplines au sens strict est majeure. II s'agit en effet non pas uniquement de constater le rôle du premier sur le second - il est massif et croissant - mais plus exactement d'identifier, de caractériser et de délimiter le type de promotion des disciplines qui peut avoir lieu par le truchement des réseaux sociaux. Nous aborderons un tel impact à la fois quantitativement et qualitativement et nous parlerons de "force de frappe " pour bien matérialiser le caractère massif et percutant de l'impact des réseaux sociaux à propos desquels il est parfois utile d'évoquer les « jeux d'ombres et de lumières sur les organisations » (Baret et al., 2006). Nous ciblerons les impacts sur les chercheurs tout d'abord puis, simultanément, sur les disciplines (une discipline n'est finalement rien d'autre qu'un groupement d'un ou plusieurs chercheurs qui s'agrègent autour des thématiques, de concepts et d'outils) et sur les institutions (une institution n'est finalement rien d'autre qu'un groupement d'acteurs qui s'agrègent autour d'une entité de jure / de facto, auxquelles ils sont rattachés administrativement, académiquement et/ou opportunément). Ces deux définitions, certes un peu descriptives, nous permettent toutefois d'avancer sur la promotion des disciplines à la fois par les réseaux sociaux et comme réseau social (Vitari et Pillet, 2019).

Qu'en est-il de la force de frappe quantitative des réseaux sociaux ? II ne suffit pas d'insister sur l'évidence de leur puissance numérique - nombre d'usagers, nombre d'abonnés, nombre de pages primo-visitées, nombre de partages de contenus, nombre de "vues", nombre d'interactions (les "like ", les " soutiens ", les " intérêts », etc.), durée de la connexion, etc. - pour montrer qu'ils ont de l'influence sur la diffusion des contenus, et donc sur la promotion des disciplines des chercheurs en sciences de gestion et du management. En effet, un internaute sur dix seulement n'est pas connecté à l'un des principaux réseaux sociaux accessibles en France. Cela signifie par conséquent que neuf internautes sur dix sont connectés et dès lors qu'ils sont accessibles - ou plus exactement éligibles - à la diffusion de contenus disciplinaires par les réseaux. La force de frappe quantitative est donc manifeste et les internautes ont accès à des contenus relayés par les réseaux et relatifs aux sciences de gestion et du management en général, et à leurs infra-disciplines en particulier. Que le dernier article publié par le professeur Payet soit accessible sur sa page Facebook ou sur son compte Research Gate n'est pas le cœur de la question ; qu'il soit lu l'est tout autant et surtout, qu'il soit partagé après avoir été lu et apprécié (ou pas). D’ailleurs, le fait de partager sans avoir lu au préalable un article est de plus en plus contrôlé, voire empêché (cas de Twitter, de WhatsApp), afin de ne pas ajouter inutilement du trafic au trafic et de ne pas créer artificiellement un buzz par le simple fait de partager du contenu! 
Qu'en est-il de la force de frappe qualitative ? C'est bien là que les exigences de pertinence et de robustesse des contenus interviennent pour envisager la promotion réelle et effective des disciplines, et non pas leur simple mise en abime numérique noyée dans le big data dont le « big » va l'emporter sur le "data ». Vitari et Pillet (2019) indiquent, à propos de la structuration de la discipline "systèmes d'information ", la force des liens croisés, le rôle des revues académiques et la puissance de la rencontre des réseaux de co-auteurs. C'est bien là qu'il convient de classer les réseaux sociaux en fonction de critères plutôt qualitatifs que quantitatifs, qui reviennent souvent à approximer la réputation ou plus exactement la e-réputation (Baret et al., 2006). Nous pouvons aborder cette contribution qualitative à la fois en partant du chercheur (i.e., de son laboratoire) et de sa réputation et/ou en partant de sa discipline de rattachement (i.e., de son association académique de rattachement) et de sa réputation. Nous parlons ainsi, à titre d'illustration, de la réputation du professeur Marc Bidan dont le laboratoire de rattachement est le LEMNA de l'université de Nantes et la discipline de rattachement est le management des systèmes d'information, fédérée par l'Association Information \& Management (AIM). Ces communautés sont parfois modestes lorsqu'elles sont prises individuellement mais elles deviennent puissantes lorsqu'elles s'agrègent ponctuellement par l'intermédiaire des réseaux (sociaux ou pas) et s'interconnectent et infusent via les nœuds de réseaux de chacune d'elles. La puissance de Linkedln est à ce titre spectaculaire.

Dans cette logique réputationnelle, il s'agira d'évoquer les abonnements, les usages, les citations, la présence numérique, les partages de contenus, les partages de liens, etc. C'est-à-dire que pour commencer, nous listons la simple présence sur les réseaux sociaux ou, plus simplement, sur les plateformes, en procédant alors à un premier partage entre d'un côté, les réseaux non professionnels (ici non-académiques) et de l'autre, les réseaux plutôt orientés professionnels (ici académiques) (tableau 1). La question qui taraude tout chercheur peut ici se résumer au travers du titre de l'article de Dutot (2021) : « Un bon chercheur, c'est quoi ? Un mauvais chercheur, c'est quoi ?». Nous pouvons au moins tenter de l'aborder au travers de sa réputation et de sa présence sur les réseaux.

Tableau 1 : Plateformes et réseaux

\begin{tabular}{|l|l|l|l|}
\hline \multicolumn{2}{|c|}{ Plateformes académiques } & \multicolumn{2}{c|}{ Plateformes non académiques } \\
\hline Sans followers & Avec followers & $\begin{array}{l}\text { Avec orientation pro- } \\
\text { fessionnelle }\end{array}$ & $\begin{array}{l}\text { Sans orientation pro- } \\
\text { fessionnelle }\end{array}$ \\
\hline $\begin{array}{l}\text { ORCID Google Scholar } \\
\text { Publons CiteULike } \\
\text { Microsoft Academic } \\
\text { Search Wikidata }\end{array}$ & $\begin{array}{l}\text { Research Gate Men- } \\
\text { dely Academia HAL }\end{array}$ & $\begin{array}{l}\text { LinkedIn Slack Discord } \\
\text { Zoom Teams Webex } \\
\text { Starleaf, clubhouse }\end{array}$ & $\begin{array}{l}\text { Twitter Facebook } \\
\text { TikTok Instagram } \\
\text { Wechat Youtube } \\
\text { Snapchat Spotify } \\
\text { Pinterest }\end{array}$ \\
\hline
\end{tabular}

Dans tous les cas, la plateforme utilisée pourra être mise à profit pour communiquer entre les acteurs et pour échanger en recherchant, certes, des échanges de fond (partage de contenus, de données, d'outils de collecte et de traitement, de financement, de collaborations, d'affiliations, de co-diplomation, de support de publication, etc. ), qui vont contribuer à faire connaître les travaux et la discipline, mais aussi parfois des échanges de forme (partage de contacts, partage de clicks, demande de vue, 
demande de transferts, demande de relais, etc.). Dans le premier cas, le chercheur et sa discipline de rattachement tenteront en général d'être valorisés par le processus même de co-construction de contenu, par exemple un papier de recherche co-rédigé par le biais d'un réseau comme Research Gate, et dans le second cas, au contraire, le chercheur et sa discipline de rattachement prennent le risque d'une réussite ou d'un échec réputationnel en instrumentalisant le réseau, par exemple la diffusion d'un questionnaire sur LinkedIn ou d'un lien URL sur Facebook, selon que le réseau sera perçu comme sérieux (LinkedIn) ou pas (Facebook). De plus, outre l'identification des réseaux sociaux qui peuvent intervenir dans la diffusion et la valorisation des disciplines et infra-disciplines des sciences de gestion et du management, il faut souligner que leur caractérisation et délimitation est importante ; elle repose à la fois sur des perceptions et des pratiques (Dutot, 2021).

Nous terminerons ce paragraphe en insistant sur la diversité des réseaux sociaux - des plateformes - et donc des usages et fonctionnalités qu'ils proposent : avec ou sans follower, avec ou sans chiffrage, avec ou sans partage de contenus, avec ou sans souveraineté, avec ou sans pérennité, etc. Il y a quand même un point commun et une idée sous-jacente, celle de collecter massivement des données et des contenus qu'ils pourront ensuite travailler, traiter et monétiser. C'est leur raison d'être, c'est leur business model même. C'est aussi la raison pour laquelle les réseaux sociaux - même les plus sérieux, professionnels et académiques - se doivent de rester gratuits pour être attractifs et héberger les interactions de leurs usagers - sans (trop de) droit de regards - plutôt que ceux de leurs clients - avec un (peu de) droit de regard - dont ils ne veulent pas! De plus, ils sont en phase avec la logique de l'open access et de la transparence des données et peuvent ainsi fragiliser les revues à accès payant en captant leur contenu (les articles), notamment par le biais des publications de pre-prints comme le propose Research Gate par exemple.

La promotion des disciplines de gestion et du management - du marketing à la comptabilité en passant par les systèmes d'information, la stratégie ou la logistique - passe avant tout par celle des chercheurs, de leurs projets et de leurs travaux, qu'ils soient pris individuellement ou collectivement. C'est dans cette logique que la promotion des disciplines au travers des réseaux sociaux est liée d'abord à l'activité numérique des chercheurs, avant celle de leur institution, même si ces dernières sont rarement inertes et mobilisent de plus en plus souvent d'excellents community managers. Il est à noter enfin que l'attractivité numérique des chercheurs va d'abord s'appuyer sur leur maitrise des contenants - les réseaux, leur fonctionnalité et leur interopérabilité - car elle semble, à court et moyen terme, plus importante que celle des contenus. Un exemple récent est celui du " son » qui apparait comme le nouvel eldorado des réseaux sociaux. En effet, nous insisterons sur le succès du " son » dans les salons vocaux propres aux échanges conversationnels entre membres du " club " comme Clubhouse justement, et la contre-attaque récente de certains autres comme Facebook (Sounbites, Hotline), Twitter, LinkedIn, Spotify et surtout Discord (Stage Channels), car il s'agit de proposer des salons vocaux pour capter le plus possible de conversations, d'interviews, de réactions, et donc d'usagers et de contenus. "Nous pensons que l'audio sera bien sûr également un média de première classe et il y a tous ces différents produits à construire sur tout le spectre ", a même déclaré Mark Zuckerberg, en avril 2021, sur un chat en groupe du réseau Discord au sein duquel il s'était invité. 
Ainsi, comme les réseaux les accueillent et les outillent, les chercheurs se regroupent, avec ou sans l'aval de leur institution, et forment des groupes fermés (page Facebook, groupe WhatsApp, groupe Linkedln, classe Slack, salon Discord, etc.), c'est-à-dire qu'ils induisent des " effets réseaux " qui à leur tour tendent à légitimer et à renforcer les plateformes qui les hébergent. Ces contenus échangés par les chercheurs en réseau vont ensuite contribuer à promouvoir leur discipline et leur thématique centrale de recherche par le trafic qu'ils vont induire, et les nouveaux usagers qui vont s'agréger. Nous avons schématisé ce processus dans les quatre étapes du tableau 2.

Tableau 2 : Les grandes étapes de l'offre de contenants à la promotion de contenus

\begin{tabular}{|l|l|l|l|}
\hline Étape 1 & Étape 2 & Étape 3 & Étape 4 \\
\hline Réseaux & $\begin{array}{l}\text { Chercheur (individu, } \\
\text { groupe formel, groupe } \\
\text { informel, communauté } \\
\text { de pratique, etc.) }\end{array}$ & $\begin{array}{l}\text { Institution } \\
\text { (laboratoire, } \\
\text { composante, société } \\
\text { savante, association, } \\
\text { etc.) }\end{array}$ & $\begin{array}{l}\text { Disciplines } \\
\text { (comptabilité, } \\
\text { contrôle, stratégie, } \\
\text { logistique, marketing, } \\
\text { etc.) }\end{array}$ \\
\hline $\begin{array}{l}\text { Offre de contenants, } \\
\text { de fonctionnalités } \\
\text { et d'outils perçus } \\
\text { comme utiles, fiables } \\
\text { et faciles }\end{array}$ & $\begin{array}{l}\text { Adoption du contenant } \\
\text { et de ses outils en } \\
\text { fonction du contenu } \\
\text { disciplinaire à } \\
\text { construire, à produire } \\
\text { et/ou à partager } \\
\text { (académique / non } \\
\text { académique, etc.) }\end{array}$ & $\begin{array}{l}\text { Adoption, correction } \\
\text { ou interdiction du } \\
\text { contenant par la } \\
\text { DSI de l'institution } \\
\text { dans la perspective } \\
\text { d'une légitimation } \\
\text { institutionnelle de } \\
\text { facto du contenu de la } \\
\text { recherche }\end{array}$ & $\begin{array}{l}\text { Promotion, } \\
\text { attractivité, } \\
\text { présence numérique, } \\
\text { e-réputation (positive } \\
\text { ou négative), } \\
\text { pérennisation, } \\
\text { transformation }\end{array}$ \\
\hline
\end{tabular}

Les « effets réseaux » observés par et pour les chercheurs en sciences de gestion et du management - notamment au travers des bouleversements induits par la pandémie de Covid-19 tels que la distanciation physique et le télétravail pluri-plateformes produisent même des pratiques, des routines et des contournements relativement intéressants qui risquent de s'installer durablement tant les enjeux de pouvoir (qui maîtrise quoi ?) et le capital social (qui parle à qui ?) sont désormais liés aux pratiques et appropriations sur et par les réseaux sociaux (Karoui et Dudézert, 2012). Nous terminerons justement par la mise en perspective du rôle des plateformes de visioconférences que nous assimilerons ici à des réseaux sociaux professionnels (Zoom, Teams, Webex, Starleaf, etc.) et certaines plateformes de gaming (Discord, Twitch, etc.) pour partager des échanges et des documents... et pour co-construire autrement leur discipline ! Ces réseaux, leur apport et leur hybridation sont même en passe de créer une discipline nouvelle. La boucle sera ainsi bouclée.

\section{Des technologies et de nouveaux horizons}

De nouveaux horizons s'ouvrent avec l'aide des nouvelles technologies pour la communauté des enseignants-chercheurs. Ils sont de trois natures : l'aide personnalisée aux candidats pour leur carrière, la détection de hauts potentiels et, en contrechamp, la détection des vulnérabilités. 


\section{L'aide personnalisée aux candidats pour leur carrière}

Pour apprendre, un modèle d'IA a besoin de données en entrées et de résultats en sorties. Promotions, attribution de la PEDR, qualifications constituent autant de bases de cas. En effet, en entrée, il y a des dossiers contenant des données et des informations; comme variable modératrice, il y a des rapporteurs ayant des caractéristiques; et en sortie, il y a des attributions ou non. Cet ensemble d'inputs et d'outputs constitue alors une base permettant à un modèle d'apprendre. L'apprentissage contribuera à faire émerger des règles de succès et des règles d'échecs. C'est là que l'aide personnalisée peut entrer en jeu. Avant d'imaginer le type de règles qui pourraient ainsi être générées, décrivons l'architecture nécessaire.

Tout d'abord, un prestataire de cloud devra être choisi. Dans l'idéal, un prestataire national de type $\mathrm{OVH}$ serait retenu. Mais, à défaut, l'un des géants du type Amazon, avec stockage des données en France, pourrait être utilisé pour la qualité de ses services de machine learning. Ensuite, il s'agira de constituer le dataset (l'ensemble des données pour l'apprentissage); il faudra alors trouver les dossiers, ajouter des métadonnées à ces dossiers (nom, date etc.), et s'assurer que le format des données contenus dans le PDF, car en général ce sont des PDF, est lisible. Sinon, il y aura une phase d'OCR pour transformer l'image en texte. Des données concernant les rapporteurs et les avis devront aussi être disponibles. L'ensemble sera alors stocké sous une forme structurée. Enfin, une analyse des documents en NLP peut être menée pour proposer d'autres métadonnées et un modèle d'apprentissage doit être créé et testé.

Ces phases sont longues et couteuses mais peuvent, d'une part, conduire à mieux comprendre les décisions prises au travers de l'émergence de règles et, d'autre part, servir de base à une prédiction du succès (ou non) d'un dossier. Donnons quelques illustrations. La mise en évidence, ou la non mise en évidence, de liens entre les disciplines des candidats et celles des rapporteurs peut conduire à des évolutions en termes de sélection des rapporteurs. Des seuils en termes de pédagogie, de recherche ou d'administratif peuvent apparaître, ceci permettrait alors de donner des indications aux candidats du type, si vous avez ce profil de publications avec cette durée de thèse vous avez $x \%$ de chances d'être qualifié. On peut même imaginer que le candidat puisse pré-tester son dossier et choisir ensuite de l'envoyer ou non. L'immédiate critique face à un tel processus serait de craindre la disparition de l'humain. Cependant, balayons une telle crainte en indiquant que le fait de connaître la manière, souvent inconsciente, avec laquelle un groupe décide permet justement à l'individu de s'améliorer, ou du moins, de se situer du point de vue de son comportement. De plus, des indications quant à la structure du contenu d'un dossier peuvent permettre de mieux voir les forces et faiblesses, et de réduire certains biais d'attention. Ainsi, l'IA possède toutes les caractéristiques pour permettre aux membres du CNU, comme aux candidats, de mieux se connaître et de se centrer sur les valeurs de chacun sans en oublier.

\section{La détection de hauts potentiels (IA et agilité digitale)}

L'IA repose sur un double socle pour que ses données proposées à l'entrée se transforment en résultats pertinents à la sortie. D'une part, elle s'appuie sur des algorithmes - donc des équations - qui en sont son côté soft et, d'autre part, elle s'appuie sur des ordinateurs puissants - donc des supercalculateurs - qui en sont son côté hard. 
La combinaison de la finesse des modélisations et suggestions mathématiques et de la puissance des serveurs et ordinateurs en réseaux explique que I'IA progresse dans tous les domaines où des données peuvent être collectées, stockées, traitées et analysées afin de proposer ce qui était jadis nommé une aide à la décision, et qui est désormais plus proche de ce que nous pourrions dénommer une suggestion pour la décision. Par exemple, en fonction des data qui auront été collectées avec ou sans votre consentement explicite, I'IA suggèrera que, comme vous avez déjà aimé regarder telle série historique et que, comme vous venez d'acheter telle voiture rouge, alors il est probable que vous apprécierez vos vacances en Italie. De même, une autre IA vous suggérera qu'après la lecture de tel article (data via Research Gate), et au regard de votre participation récente à telle conférence (data via Science Conf), alors il y a de fortes chances que vous soyez intéressé par la sortie de tel numéro spécial de cette fameuse revue académique (data via Google Scholar ou Scopus, voire Cairn).

Nous pouvons extrapoler cette analyse avec la détection des talents ou plus exactement des " hauts potentiels » dans une logique académique ou disciplinaire. La question n'est pas de collecter les données - elles sont largement disponibles au regard de la présence numérique de la plupart des enseignants chercheurs et chercheurs - mais plutôt de les traiter et de les combiner entre elles pour " sortir » un résultat pertinent, c'est-à-dire un chercheur à haut potentiel ! Ainsi, la question sera avant tout " qu'est-ce qu'un chercheur à haut potentiel ? ", et comment une IA peut-elle tenter de le caractériser. Si la réponse est, par exemple, « un chercheur qui serait (encore) jeune mais aussi (déjà) publiant, (suffisamment) intégré, (marginalement) fantaisiste et (significativement) productif ", alors il sera aisé d'en dégager un profil-type en termes de data et de caractérisation. Certes, ces qualifications de " hauts potentiels " peuvent être incomplètes, elles sont très souvent insatisfaisantes et cachent une réalité plus complexe. Reprenons notre exemple et proposons un tableau 3 qui pourrait détecter et identifier les cibles.

Tableau 3 : Critères quantitatifs et critères qualitatifs des hauts potentiels

\begin{tabular}{|c|c|c|c|c|c|}
\hline $\begin{array}{l}\text { Critère } \\
\text { qualitatif }\end{array}$ & Encore jeune & Déjà publiant & $\begin{array}{l}\text { Suffisamment } \\
\text { intégré }\end{array}$ & $\begin{array}{l}\text { Marginalement } \\
\text { fantaisiste }\end{array}$ & $\begin{array}{l}\text { Significative- } \\
\text { ment productif }\end{array}$ \\
\hline $\begin{array}{l}\text { Critère } \\
\text { quantitatif } \\
\text { strict } \\
\text { pour les hauts } \\
\text { potentiels } \\
\text { finalisés }\end{array}$ & $\begin{array}{l}\text { Moins } \\
\text { de } 30 \text { ans }\end{array}$ & $\begin{array}{l}\text { Plus de cinq } \\
\text { articles } \\
\text { rang } 1 \text { ou } 2 \\
\text { FNEGE / CNRS } \\
+ \text { Indice } \\
\text { Research Gate } \\
\text { supérieur à } 20\end{array}$ & $\begin{array}{l}\text { Membre } \\
\text { d'au moins } \\
\text { un bureau } \\
\text { (association } \\
\text { académique, } \\
\text { laboratoire, } \\
\text { board) }\end{array}$ & $\begin{array}{l}\text { Activité com- } \\
\text { plémentaire } \\
\text { sur au moins } \\
\text { une journée } \\
\text { par semaine } \\
\text { (sport, art, vie } \\
\text { associative, vie } \\
\text { politique, etc.) }\end{array}$ & $\begin{array}{l}\text { Plus de trois } \\
\text { supports de } \\
\text { publication dif- } \\
\text { férents autres } \\
\text { que les revues } \\
\text { classées (The } \\
\text { Conversation, } \\
\text { La Tribune, } \\
\text { HBR France, } \\
\text { ASAP, etc.) }\end{array}$ \\
\hline $\begin{array}{l}\text { Critère quan- } \\
\text { titatif souple } \\
\text { pour les hauts } \\
\text { potentiels in } \\
\text { progress }\end{array}$ & $\begin{array}{l}\text { Moins } \\
\text { de } 40 \text { ans }\end{array}$ & $\begin{array}{l}\text { Plus de trois ar- } \\
\text { ticles rang } 1 \text { ou } \\
2 \text { FNEGE/ CNRS }\end{array}$ & $\begin{array}{l}\text { Sympathisant } \\
\text { d'au moins } \\
\text { un bureau } \\
\text { (association } \\
\text { académique, } \\
\text { laboratoire, } \\
\text { board) }\end{array}$ & $\begin{array}{l}\text { Activité com- } \\
\text { plémentaire } \\
\text { sur au moins } \\
\text { une journée } \\
\text { par mois } \\
\text { (sport, art, vie } \\
\text { associative, vie } \\
\text { politique, etc.) }\end{array}$ & $\begin{array}{l}\text { Plus d'un } \\
\text { support de } \\
\text { publication } \\
\text { différent autre } \\
\text { que les revues } \\
\text { classées (The } \\
\text { Conversation, } \\
\text { La Tribune, } \\
\text { HBR France, } \\
\text { ASAP, etc.) }\end{array}$ \\
\hline
\end{tabular}


Cependant, il est possible d'être encore plus efficace dans le ciblage et le repérage de ces hauts potentiels en remarquant que ces derniers, à l'issue de leurs thèses, qu'ils ont soutenues dans de grands laboratoires avec des jurys plutôt senior, sont " souvent " : (1) qualifiés par le CNU dès leur première candidature ; (2) en poste au sein d'un IAE des grandes métropoles (Paris, Lyon, Lille, Toulouse, Aix-en-Provence, etc.) ou dans une business school située en haut du tableau (top 5 / top 10), voire en poste comme associate professor dans les grandes universités anglo-saxonnes (Londres, Berlin, New York, Sydney, Los Angeles, etc.) ; et (3) largement suivis par et sur les réseaux sociaux. Ce qui nous donnerait une dernière ligne à ajouter avec un menu déroulant qui permettrait aux algorithmes de repérer et puis surtout de hiérarchiser les hauts potentiels qui seront identifiés (tableau 4).

Tableau 4 : Critères complémentaires des hauts potentiels

\begin{tabular}{|l|l|l|l|l|l|}
\hline $\begin{array}{l}\text { Critère } \\
\text { qualitatif }\end{array}$ & Encore jeune & Déjà publiant & $\begin{array}{l}\text { Suffisamment } \\
\text { intégré }\end{array}$ & $\begin{array}{l}\text { Marginalement } \\
\text { fantaisiste }\end{array}$ & $\begin{array}{l}\text { Significativement } \\
\text { productif }\end{array}$ \\
\hline $\begin{array}{l}\text { Critère } \\
\text { quantitatif } \\
\text { complémen- } \\
\text { taire pour } \\
\text { hiérarchiser } \\
\text { les hauts } \\
\text { potentiels }\end{array}$ & $\begin{array}{l}\text { Quel âge } \\
\text { avez-vous? }\end{array}$ & $\begin{array}{l}\text { Combien } \\
\text { d'articles } \\
\text { de rang 1, } \\
\text { de rang 2, } \\
\text { de rang 3 } \\
\text { ou 4? }\end{array}$ & $\begin{array}{l}\text { Combien de } \\
\text { co-auteurs } \\
\text { en moyenne } \\
\text { sur les } \\
\text { articles } \\
\text { de rang 1, } \\
\text { de rang 2? }\end{array}$ & $\begin{array}{l}\text { Tollowitter, Linke- } \\
\text { dln ? Combien } \\
\text { de postes déjà } \\
\text { occupés ? } \\
\text { Combien de dis- } \\
\text { tinctions reçues } \\
\text { autres qu'aca- } \\
\text { démiques? }\end{array}$ & $\begin{array}{l}\begin{array}{l}\text { Combien de } \\
\text { projets en cours } \\
\text { de production : } \\
\text { articles? études? } \\
\text { autres projets? }\end{array} \\
\text { anRT }\end{array}$ \\
\hline
\end{tabular}

Il est donc intéressant de noter que la question centrale n'est pas le " comment " détecter (les data sont à disposition et aisément analysables grâce à des logiciels et équations dédiés), mais plutôt le " quoi » détecter : les cerveaux sur-stimulés, la minutie dans l'exécution des tâches, la rapidité et la pertinence dans la production, le décalage avec "la norme et les normaux ", etc. Mais finalement, si "nous » nous trompions sur ce qu'est un enseignant-chercheur à haut potentiel ? Si des critères comme l'âge ou les publications, voire les postes occupés, étaient de plus en plus discutables, notamment au regard des évolutions profondes du métier même d'enseignant-chercheur. La simple production de connaissance n'est pas suffisante, il faut la diffuser, la communiquer, la vulgariser... et la raconter ! Le métier d'enseignant-chercheur a profondément changé et s'est transformé. Produire ne suffit certes plus et les débats sur le " nouveau métier de chercheur " sont encore largement ouverts, tant sa caractérisation reste délicate (Monin, 2017 ; Garreau et de Montmorillon, 2019). Toutefois, les hauts potentiels senior sont de plus en plus intéressants à analyser avec une expérience qui infuse et leur permet d'être à la fois pertinents et productifs en seconde partie de carrière (Monin, 2017), et qui ont à leur disposition des outils numériques qui leur permettent de bien diffuser leurs travaux, mais pas forcément dans le cadre de revues scientifiques ou de conférences académiques.

Nous conclurons en renvoyant à la nuance et à l'impératif d'humilité face à des équations dont bien souvent la transparence, et donc la redevabilité, est discutable mais qui font face, elles aussi, à des évolutions rapides des compétences managériales qu'elles ont à débusquer, à identifier, à évaluer (Giraud et al., 2021). La littérature 
évolue assez vite et nous donne ainsi l'occasion de documenter le terrain IA / hauts potentiels qui reste sensible car il renvoie à notre métier, à son sens, ses implications, à ses contournements aussi, et à sa profonde mutation toujours en cours, et pour encore de nombreuses années (Garreau et de Montmorillon, 2019).

\section{La détection des vulnérabilités (IA et mort digitale)}

Une autre question sensible et ouverte doit être abordée : celle de la détection des vulnérabilités. Toutefois, le " pourquoi détecter » ne se pose pas de la même façon que dans le paragraphe précédent tant la détection des vulnérabilités est un enjeu majeur en ces temps perturbés, notamment par la crise sanitaire et ses conséquences sociétales et économiques. À nos yeux, en effet, si détecter les hauts potentiels est une possibilité, détecter les vulnérabilités est un impératif. Nous parlerons bien évidemment, car c'est l'objet du présent ouvrage, des vulnérabilités des chercheurs et enseignants-chercheurs dans le cadre de l'exercice des trois dimensions de leur métier, la recherche, l'enseignement et l'administration, et exclusivement dans ce cadre-là. Les données accessibles et mises à disposition des équations et calculateurs des modèles d'IA sont nombreuses tant le numérique a investi le champ de ces métiers (publications académiques, pre-prints, réseaux sociaux, conférences, sociétés savantes, universités, publications non académiques, etc.). Cela permet de traiter une masse de données significative et régulièrement renouvelée au sein des communautés (Vitari et Pillet, 2019).

Les vulnérabilités et autres " risques du métier " peuvent être directement liés à l'exercice du travail durant les activités de recherche ou d'enseignement, par exemple en laboratoire pour les disciplines exposées (physique, chimie, biologie, mécanique, etc.), avec des accidents de laboratoire comme des explosions, des chocs, des incendies, etc., mais aussi en séance pour des disciplines moins exposées aux expérimentations dangereuses comme c'est le cas des SHS avec des accidents comme des chutes, des altercations, des agressions verbales et/ou physiques, etc. Dans ce cas, grâce aux données collectées par le passé dans des situations similaires, I'IA sera utile dans une logique préventive et dans la perspective d'éviter de reproduire des situations telles que l'accident aurait de forte de chance de se produire. C'est toute la logique de la sécurisation des protocoles expérimentaux dans le cadre des expérimentations en sciences dites dures mais aussi de la détection des situations ou des enjeux sensibles - sur les réseaux sociaux, par exemple - dans le cadre de cours en SHS qui abordent des sujets à dimension potentiellement polémiste.

Les vulnérabilités peuvent être indirectement liées à l'exercice du métier mais plutôt à l'accumulation de stress, d'injonction parfois contradictoires et d'autres sollicitations liées à la carrière. Nous évoquerons ici à la fois le cas de la recherche et de la production scientifique, qui sous-tendent largement les promotions et les évolutions de carrière de l'enseignant chercheur, et le cas de l'enseignement et des responsabilités administratives, qui sous-tendent son ancrage local, notamment avec l'avènement rapide de distances sociales et du télétravail dans des conditions technologiques et opérationnelles le plus souvent plutôt subies que pilotées. La petite " mort digitale ", c'est-à-dire une sorte de lâcher prise digital, est alors détectable par I'IA dès lors que l'enseignant-chercheur est moins présent et moins actif - régularité des connexions, temps moyen de connexion, lieu des connexions, temps 
moyen entre deux connexions, temps de réponse, nombre de mots par réponse, usage intempestif de " ! ", " ... » ou de " majuscules », etc. - sur les plateformes qu'il avait l'habitude de fréquenter professionnellement (Research Gate, LinkedIn) ou non professionnellement (Facebook, Twitter). Le tableau 5 peut aider à schématiser le rôle de I'IA dans la détection des vulnérabilités en montrant qu'elle est utile plutôt ex post dans le cadre de l'exercice direct du métier et qu'elle le sera plutôt ex ante dans un cadre plus indirect.

Tableau 5 : Vulnérabilités, données et contextualisation

\begin{tabular}{|l|l|l|}
\cline { 2 - 3 } \multicolumn{1}{c|}{} & $\begin{array}{l}\text { Vulnérabilité liée directement } \\
\text { au métier }\end{array}$ & $\begin{array}{l}\text { Vulnérabilité liée indirectement } \\
\text { au métier }\end{array}$ \\
\hline $\begin{array}{l}\text { Données (âge, ancienneté, } \\
\text { institution, publications, } \\
\text { sollicitations, présence } \\
\text { numérique, citation, etc.) }\end{array}$ & Pérennes (en années) & $\begin{array}{l}\text { Fraiches } \\
\text { (en jour, semaine, mois) }\end{array}$ \\
\hline Sources & $\begin{array}{l}\text { Réseaux académiques Réseaux } \\
\text { professionnels Plateformes } \\
\text { scientifiques Signaux forts }\end{array}$ & $\begin{array}{l}\text { Réseaux sociaux PQR Presse } \\
\text { professionnelle Signaux faibles }\end{array}$ \\
\hline Contribution de l'IA & Ex post & Ex ante \\
\hline Type de vulnérabilités & $\begin{array}{l}\text { Accident Agression Stigma- } \\
\text { tisation Fatigue physique et } \\
\text { mentale }\end{array}$ & $\begin{array}{l}\text { Décrochage Harcèlement Perte } \\
\text { de confiance Perte de motiva- } \\
\text { tion Perte d'agilité digitale }\end{array}$ \\
\hline Conséquences à terme & Fragmentation digitale & Mort digitale \\
\hline
\end{tabular}

La complexité de la question de la vulnérabilité et de sa détection par I'IA tient aussi à la problématique du " changement " profond du métier d'enseignant-chercheur qui est notable et documenté depuis quelques années, et notamment depuis les années 2010 (Garreau et de Montmorillon, 2019). Notons, par exemple, que trois tendances lourdes parmi d'autres, la fragmentation des tâches, la précarisation perçue du corps professoral et l'isolement institutionnel, peuvent expliquer la montée des vulnérabilités en termes de décrochage digital. Elles sont aisément détectables par les IA à partir des data modélisant la présence et l'activité des collègues sur Internet ainsi que leur indicateur de performance professionnelle et autres tableaux de bord proposés par chacune des applications et plateformes. De plus, ces décrochages digitaux - pour le moment, ils ne sont que digitaux - ont été largement accentués par la crise sanitaire et ses conséquences sociétales durant les confinements successifs depuis mars 2020 (distanciation, plateformisation, visioconférence, etc.). Il est intéressant de souligner que le " nombre de vues " d'un contenu ou d'un post n'est plus accessible - trop anxiogène et contreproductif selon les analystes sur Twitter et ne le sera plus à terme sur Facebook ou Linkedln. En bref, les traces ne sont détectables par les IA que si elles sont numériques et donc collectables sous forme de données. II faut donc agir et intervenir, avec ou sans I'IA, dès que les signaux faibles du décrochage des collègues sont perçus. 


\section{Conclusion}

CNU et numérique sont sources de fortes synergies : des synergies pour améliorer l'existant, et des synergies pour mettre en avant de nouvelles missions que le CNU pourrait prendre en charge, tester et déployer. Mais le monde du numérique pourrait aussi bénéficier de l'emploi par le CNU de tels outils et de leurs pratiques. En effet, des entreprises ou des organisations pourraient proposer des solutions adaptées, puis se servir de cette validation nationale pour améliorer le fonctionnement des universités, des IAE, des IUT et des business schools. Les opportunités sont là et le contexte lié à la Covid-19 est propice aux changements. Gageons que l'avenir nous montrera combien certaines de ces opportunités pourront et sauront être saisies. 


\section{L’héritage universitaire pour les jeunes de demain}

Anne Goujon Belghit et Jocelyn Husser

Les universités garantissent six missions de service public aux citoyens désireux d'accéder à l'enseignement supérieur d'après la loi n²013-660 du 22 juillet 2013. La première mission consiste à assurer la formation aux citoyens français qui viennent de réussir leur baccalauréat ou aux professionnels désireux de retourner se former. La deuxième mission désigne les activités scientifiques afin de s'interroger sur les problématiques sociales, sociétales ou de développement durable, et de partager le fruit de ces réflexions avec les étudiants et plus largement, la société. La troisième mission concerne l'ascension sociale ainsi que l'insertion professionnelle afin d'assurer une adéquation optimale entre les futurs professionnels formés par l'université et les besoins des organisations. La quatrième mission vise à diffuser massivement par différents canaux la connaissance scientifique émergente et à la vulgariser pour la rendre accessible, susceptible de se traduire par une performance économique, sociale ou sociétale. La cinquième mission interroge la possibilité pour les universités de participer à la construction de l'espace européen en pensant notamment les enseignements en termes d'ECTS (European credits transfer system) pour favoriser les équivalences entre pays européens et promouvoir les échanges universitaires. La sixième et dernière mission désigne la coopération internationale.

Depuis les années 2000, les universités subissent des transformations radicales modifiant les logiques de fonctionnement, les modalités de financement et les possibilités d'action des acteurs travaillant pour ces structures. La grille de lecture de Crozier et Friedberg (1977), que l'on retrouve dans leur œuvre majeure, L'acteur et le système, apporte un éclairage intéressant afin de comprendre les enjeux de pouvoir dans les systèmes d'action concrets que sont les universités, pour des acteurs qui s'approprient de nouvelles règles et découvrent de nouvelles zones d'incertitude. Nous proposons dans le chapitre d'investiguer la question du devenir des universités françaises à travers un portrait de l'évolution du statut des universités qui s'appuie sur notre propre expérience en tant qu'acteur universitaire. La parole est ensuite donnée à Marianne, étudiante et actrice " du futur " puis à Jean, enseignant-chercheur et acteur du futur, afin d'évoquer les difficultés auxquelles peuvent se préparer 
les nouvelles générations évoluant dans les systèmes d'action concret universitaires. Nous terminons par une rencontre entre Marianne et Jean pour identifier des leviers susceptibles d'améliorer en profondeur nos universités.

\section{Évolution du statut des universités}

L'université joue un rôle primordial dans une société puisqu'elle contribue au niveau de formation de la population d'une nation. Le $x^{\mathrm{e}}$ siècle est marqué par deux grandes périodes clés. La première partie du siècle propose une éducation supérieure à des " happy fews ", qui appartiennent à une catégorie socio-professionnelle privilégiée. La deuxième partie est marquée par la révolution de mai-68, qui modifie les règles universitaires, proposant désormais une éducation pour tout citoyen français, transcendant l'approche élitiste et accueillant plus largement la population française (Prost, 1989). Ce changement d'approche modifie les règles de fonctionnement universitaire ainsi que les budgets que l'État doit affecter à ses universités. Les capacités d'accueil augmentent et l'équipe d'enseignants-chercheurs grandit. De même, les critères d'excellence évoluent concernant les attentes du métier et des compétences détenues par ses enseignants-chercheurs. Pour autant, la rémunération affectée à ces corps de métier n'est plus en correspondance avec l'accroissement de ces exigences et des responsabilités qui leur sont confiées (Goujon Belghit et Husser, 2021). Les acteurs universitaires apprennent de nouvelles règles, répondent à l'exigence croissante de la recherche, demeurent disponibles pour les étudiants et leurs institutions, mais déplorent le manque de reconnaissance sociale.

Le modèle de la formation universitaire pour tous est aujourd'hui profondément remis en question. L'approche est indirecte, quasiment invisible pour les citoyens français. Seuls les enseignants-chercheurs souffrent, pour le moment, de la situation, les étudiants n'en ressentiront les effets que dans quelques années, lorsqu'ils s'apercevront que les frais universitaires auront augmenté de manière conséquente, excluant ainsi une partie de la population. En tant qu'enseignants-chercheurs des universités, nous témoignons aujourd'hui de ces mutations, annoncées lors du traité de Lisbonne de 2000, entérinées avec la loi LRU ou loi Pécresse de 2007, ambitionnant d'accorder l'autonomie aux universités en leur transférant la gestion des budgets, des ressources humaines ainsi qu'en leur cédant la propriété de leur biens fonciers (Santini et Woerth, 2008). Le modèle universitaire reposait sur l'allocation des ressources nécessaires à leur fonctionnement par l'État afin de garantir l'accès aux citoyens français à l'éducation supérieure nationale. Depuis 2007, les universités cherchent d'autres modalités de fonctionnement étant donné le désengagement progressif de l'État, les plongeant dans la fragilité financière. Comment fonctionner sans ressources?

Les universités restent discrètes sur ces questionnements et réfléchissent afin de trouver des solutions alternatives. Ainsi, de nouveaux contrats émergent, comme les contrats LRU, qui précarisent le statut d'enseignant-chercheur, et nous observons que certains collègues assurent la même charge de travail que nous, pour une rémunération inférieure, insuffisante et sans garantie de continuité de relation d'emploi malgré leur engagement. Les gouvernances universitaires exigent désormais que les enseignants-chercheurs ne puissent pas aller au-delà du doublement 
de leur service sans pour autant engager des réflexions autour de la revalorisation des salaires, bien souvent insuffisants pour assurer une vie de famille. L'argument avancé repose sur l'idée qu'un enseignant-chercheur ne peut assurer une recherche de qualité s'il consacre trop de temps à la formation. Pourtant nous constatons que nos collègues "publiants » sont les premiers ciblés par cette mesure. Cela n'a rien de surprenant si l'on considère que l'université représente un lieu d'échanges sur les connaissances scientifiques entre académiques, mais aussi entre académiques et étudiants. Ces derniers recherchent de tels débats qui s'appuient sur les travaux scientifiques en cours.

Les enseignants-chercheurs les plus demandés sont donc naturellement ceux qui publient le plus. Cette contrainte oblige certaines composantes à fermer des formations, faute de pouvoir assurer les enseignements à un niveau de qualité suffisant, par manque d'enseignants qualifiés " disponibles ". Les maquettes des programmes de formation ont été reprises pour diminuer les volumes horaires par formation, sans diminuer pour autant les frais d'inscription, bien au contraire. La gouvernance explique qu'il faut privilégier le recrutement de professionnels afin d'assurer les enseignements qui ne peuvent plus être assumés par des enseignants-chercheurs, sauf qu'en sciences de gestion et du management, tous les programmes intègrent de longue date des professionnels. Les IAE et les IUT sont depuis toujours convaincus de l'importance du rapprochement du monde professionnel et du monde de l'enseignement. Comment devenir de bons professionnels sans liens avec le monde réel ? Ainsi, depuis les années 1950, les IAE de France assurent des formations aux professionnels en respectant leurs contraintes et la conduite d'une double vie conjointe : vie professionnelle et vie d'apprenant. Depuis leur création, les IAE promeuvent un enseignement assuré par un binôme académique et professionnel.

Comment expliquer aujourd'hui la diminution des heures de formation, cette contrainte d'heures d'enseignement imposée aux collègues sans remettre en question la qualité de la formation universitaire ? Nous pouvons nous demander si l'intention n'est pas d'appliquer la politique de la terre brulée, qui consiste à montrer que le système est à bout en l'asphyxiant, et en montrant qu'il n'existe pas d'autre choix que de fermer les structures ? S'il s'agit de la volonté de nos politiques, pourquoi ne pas l'annoncer clairement aux citoyens français ? Le paradigme de l'enseignement supérieur pour tous s'effondre au profit d'un enseignement dispensé à une population capable de payer à la suite du désengagement de l'État qui ne finance plus ses universités.

Cette politique du « un, deux, trois, soleil » se révèle dans les décisions surprenantes de l'État, comme celle d'annoncer la fin de la qualification aux fonctions de professeur des universités le premier jour du deuxième confinement, en pleine crise de la Covid-19. Cela se perçoit également lorsque le ministère de l'Enseignement supérieur s'adresse directement aux candidats sans informer au préalable les membres du CNU. L'objectif semble clair : faire disparaître toute instance nationale qui constitue aujourd'hui un obstacle à l'autonomie des universités, c'est-à-dire au désengagement de l'État. En tant qu'enseignant-chercheur convaincu de l'importance de l'éducation de nos citoyens français, que ce soit en début de carrière professionnelle ou en cours de carrière, nous nous interrogeons sur le modèle d'éducation supérieure de demain. 


\section{Quelle université demain? Témoignage de Marianne}

Nous proposons de narrer l'histoire de Marianne, actrice du nouveau modèle universitaire. Fille d'une famille " populaire » qui envisage après son baccalauréat des études supérieures, elle n'est pas la meilleure de sa classe mais fait partie de la première moitié de sa promotion. Marianne ne peut donc prétendre à une bourse pour poursuivre ses études supérieures et doit sérieusement envisager l'université proche de son domicile pour ne pas avoir à payer un loyer en plus de son inscription. Le prix de la formation à l'année est de 7000 euros, pour suivre une licence en sciences de gestion et du management. Elle ne peut malheureusement pas débourser une telle somme d'argent ni demander à ses parents de contracter un prêt. Pourtant, elle aimerait pouvoir suivre des études, pour ce prix, sur cinq années. Le début du cauchemar commence alors car elle ne souhaite pas renoncer à son rêve mais doit affronter la dure réalité liée aux réformes universitaires.

Marianne opte ainsi pour une formation " à la carte ", ne pouvant débourser la somme nécessaire pour suivre le programme dans son intégralité. La formation à la carte est défendue par l'État comme modalité d'enseignement autorisant l'étudiante ou l'étudiant à suivre des cours de disciplines différentes, assurant ainsi la transversalité dans l'accès aux enseignements. Cette modalité aurait pu intéresser Marianne si elle avait pu lui garantir l'acquisition de compétences débouchant sur une expertise complète, recherchée sur le marché de l'emploi par des entreprises. En effet, elle ambitionne de rejoindre une direction de gestion des ressources humaines pour accompagner les salariés d'une entreprise dans leur trajectoire professionnelle, en proposant des formations adaptées aux besoins de chacun. Elle décide de suivre plusieurs modules en management pour un volume horaire total de 100 heures et un coût total de 2000 euros. Le volume total horaire de la formation en management étant de 350 heures, elle doit renoncer à 250 heures de formation.

Elle commence donc son cursus d'étudiante en management par un cours d'introduction générale aux sciences de gestion et du management assuré par un chargé de TD qui est en première année de doctorat. II manque clairement d'expérience en enseignement et les prises de note sont difficiles car Marianne a du mal à comprendre l'enseignant, qui passe en revue les concepts principaux sans les expliquer. Il conseille de lire attentivement les ouvrages en management écrits par des professeurs des universités, qui ne sont plus sollicités pour dispenser ce type d'enseignement. Ils ont été remplacés par des vacataires, moins chers pour les universités. Les enseignants vacataires " low cost » assurent la plupart des enseignements universitaires car les enseignants-chercheurs ont été engagés par les écoles de commerce. Pour Marianne, il est impossible d'envisager de s'inscrire en école de commerce ; l'université représente sa seule option pour poursuivre des études supérieures, même si la formule de formation " à la carte » rend plus difficile l'obtention d'un diplôme, qui nécessite de valider un certain nombre de compétences clés.

$\mathrm{Au}$ bout de cinq années, Marianne a réussi à valider complètement sa licence et espère très prochainement pouvoir accéder à son deuxième cycle. Elle apprend malheureusement que le second cycle de son université ferme par manque de moyens financiers et qu'elle doit s'inscrire pour l'année prochaine dans une autre université située à plus de $200 \mathrm{~km}$ de son domicile. Lors d'une soirée étudiante, elle rencontre un jeune docteur, Jean, qui a suivi des études universitaires en sciences de 
gestion et du management quelques années avant elle, lorsque " l'ancien système égalitariste " existait toujours. Jean explique qu'il se serait retrouvé dans la même situation qu'elle et n'aurait certainement pas eu les moyens financiers de réussir ses études. Pourtant, aujourd'hui, il publie dans les revues classées rang 1 FNEGE en gestion des ressources humaines, plus spécifiquement sur l'étude de la qualité de la relation à l'emploi. II lui raconte alors son parcours professionnel.

\section{Quel enseignant-chercheur demain? Témoignage de Jean}

Jean se souvient de tout. II vient de soutenir brillamment son doctorat en sciences de gestion et du management, il a un dossier solide qu'il ne peut désormais plus valoriser par le CNU, qui a cessé d'exister. II souhaite intégrer une université pour devenir maître de conférences mais il se demande comment il va parvenir à se différencier des autres docteurs de sa discipline, section scientifique 06 du CNU, car les critères de recrutement sont plutôt obscurs, difficiles à saisir de manière objective. II candidate donc auprès de différentes universités, désireux de partager ses connaissances avec des étudiants. II sait que sa candidature est sérieuse puisqu'il a déjà publié un article rang 1 FNEGE, un article rang 2 et deux articles rang 4 durant sa thèse. Sa thèse a été primée par la FNEGE et il a déjà participé à l'organisation du colloque de l'Association francophone de gestion des ressources humaines (AGRH) dans son université d'origine.

Sur les 15 candidatures qu'il dépose, Jean est convoqué à deux entretiens et finalement retenu pour un poste de maître de conférences en sciences de gestion et du management à l'Université Côte d'Azur. On lui explique que l'université ne recrute que des profils d'excellence et il se sent fier d'avoir réussi cette étape cruciale dans sa vie d'enseignant-chercheur. II a le sentiment que la vie lui sourit professionnellement, mais aussi personnellement, car il vient d'être papa d'une petite fille. Cependant, au bout de quelques mois de collaboration avec son université, il s'aperçoit que son salaire ne suffit pas à faire vivre sa famille. II compare son bulletin de paie avec la femme de ménage de ses parents et reste stupéfait de constater le peu d'écart de rémunération. Elle perçoit 1300 euros par mois et lui 1600 euros. C'est nettement insuffisant pour payer le prêt qu'il a contracté pour acheter sa maison de $90 \mathrm{~m}^{2}$ à $50 \mathrm{~km}$ de Nice et assurer une vie confortable à sa femme et à son enfant. II décide alors de reprendre contact avec son directeur de thèse pour lui demander conseil.

Ce dernier lui propose de se rapprocher d'une grande école de commerce susceptible de lui proposer un contrat de travail intéressant. Sans grande conviction, Jean contacte cette école qui, effectivement, se réjouit de l'intégrer dans ses locaux et de lui proposer non seulement une rémunération intéressante mais également des conditions de travail optimales. II s'étonne d'une telle différence de traitement et on lui répond que les réformes universitaires conduites à partir de 2009 ont contribué à désengager l'État dans le financement des universités et que les frais de scolarité demandés aux étudiants, bien qu'importants, ne suffisent pas à financer des salaires décents pour l'équipe d'enseignants-chercheurs ni d'assurer les ressources nécessaires pour doter correctement des équipes de recherche. II est vrai que 220 euros par enseignant-chercheur et par an pour assurer une activité de recherche, une dynamique de communication dans des colloques ou de lancer des expériences 
sur le terrain limite l'éventail d'action et génère chez Jean un dissentiment cognitif auquel il ne peut mettre aucune articulation pratique.

Les ressources proviennent désormais du secteur privé et les contrats de recherche répondent à des impératifs de résultat limités dans le temps; les entreprises souhaitent obtenir un livrable, c'est-à-dire un retour rapide sur investissement. La recherche fondamentale n'est plus qu'un lointain souvenir et seule la recherche appliquée, et " orientée », est désormais financée. L'enseignant-chercheur y consacre la plupart de son temps à trouver des financements au détriment de la recherche ou de son activité d'enseignement. II demeure frustré de se voir devenir un enseignant-chercheur administratif... justement happé par les dossiers administratifs à remplir, les stages de Licence 3 à valider, les visites d'entreprise à organiser pour le suivi des apprentis. Jean ressent constamment une tension entre les raisons premières qui ont motivé son choix de travailler à l'université et la réalité du quotidien.

Très rapidement, Jean découvre que l'école de commerce accorde des primes à la publication qui contribuent largement à améliorer son quotidien et lui confèrent une reconnaissance pour son engagement et ses efforts. Il voyage à travers le monde car il intervient dans les nombreuses antennes de l'école. Lors de ses voyages, il échange avec des enseignants-chercheurs américains qui lui proposent de collaborer afin de publier dans des revues de premier plan sur la scène internationale. Jean se réjouit de ces nouvelles opportunités. À cette occasion, il remarque que les conditions d'emplois de ses collègues américains sont plus favorables que les siennes. Au bout de quelques années, de collaboration fructueuse, il se voit proposer un contrat par une université américaine. II accepte cette proposition avec amertume car il ne peut s'empêcher de penser qu'il aurait préféré continuer de travailler pour la France étant donné qu'elle a contribué à l'aider à acquérir une expertise pointue, valorisable sur le marché de l'emploi à l'échelle internationale.

\section{Et si on agissait aujourd'hui? Les propositions de Jean et Marianne}

Les histoires projectives de Jean et de Marianne présentent l'intérêt de montrer la trajectoire qu'emprunte aujourd'hui la France et soulignent qu'il est urgent de réagir. Il est navrant de constater que les alertes nombreuses lancées par les collègues enseignants-chercheurs ne trouvent pas d'écho au niveau de notre gouvernement ou auprès des étudiants. Pourquoi contribuer à l'appauvrissement de notre pays en diminuant les ressources allouées à l'éducation de nos jeunes et de manière plus large de nos citoyens?

Ces « nouvelles approches », prônées par le courant du new public management à partir des années 1980 (Hood, 1991 ; Pollitt, 2009), affirment que le management public est à bout de souffle. II semble urgent de le réformer et d'intégrer des outils de pilotage venant du secteur privé. De manière concomitante, le traité de Maastricht sécurise les États européens et redéfinit les frontières nationales à l'échelle européenne. Le marché unique implique que les États n'ont plus de raison de garder le contrôle de certains secteurs clés de leur économie, comme la santé, l'éducation nationale, l'énergie, le transport, la sécurité, etc. II s'agit de transférer les secteurs publics à des acteurs privés, capables a priori de mieux prendre en 
charge les besoins des citoyens qui deviennent des clients, consommateurs, mais toujours contribuables aux deniers de l'État. Ces approches, entérinées par le traité de Lisbonne en 2000, puis portées par la loi LRU de 2007, ne se fondent sur aucune donnée empirique ; il s'agit d'une idéologie qui conduit aujourd'hui notre pays à se désengager de l'éducation nationale supérieure. Ainsi, le métier d'enseignant-chercheur se détériore lentement (Gatignol, 2014), au détriment de nos étudiants.

Quelles sont les propositions de Jean et Marianne? Jean considère que les logiques de pilotage du secteur privé ne peuvent convenir pour valoriser une activité publique, qui ambitionne de répondre aux besoins des citoyens, et pas nécessairement à engendrer des bénéfices. Notre système, fondé sur une philosophie de redistribution des ressources nationales pour venir en aide au peuple, participe à réduire les écarts entre les différentes catégories socio-professionnelles et à former une population capable de répondre aux enjeux de la concurrence internationale. Marianne pourrait ainsi suivre des études supérieures et envisager une progression sociale grâce à l'université. Les institutions publiques jouent un rôle fondamental et peuvent se montrer force de proposition pour produire des indicateurs de performance capables de traduire les enjeux sociétaux. Les outils retenus ambitionnent de diminuer les coûts de fonctionnement au détriment de la finalité sociétale de ces organisations. Pourtant, des alternatives existent.

Jean constate que les universités s'évaluent en termes de performance en fonction du classement de Shangaï. Or, de nombreuses disciplines ne peuvent prétendre à figurer dans celui-ci étant donné les critères retenus : comment publier dans Nature quand on travaille en sciences de gestion et du management ? Jean connait pourtant plusieurs recherches qui soulignent la diversité des stratégies retenues par les universités, combinant des critères quantitatifs (notamment de publications) avec des critères qualitatifs (fondés sur la réputation de l'établissement dans sa zone de chalandise) (Paradeise et Thoenig, 2013). D'autres chercheurs proposent d'intégrer des indicateurs de performance sociétale pour évaluer la performance globale spécifique aux universités (Savall et al., 2019). Les critères retenus par le gouvernement reposent sur l'idée que les universités doivent produire une connaissance se traduisant par une innovation susceptible d'être brevetée (Guillaume et Macron, 2007). À nouveau, les universités pourraient proposer des solutions pour aider les chercheurs à déposer leurs brevets. II n'est pas possible d'opposer recherche fondamentale et recherche appliquée : le brevet est la résultante d'un prisme plus large issu de la recherche fondamentale. Le brevet, tel une fleur, se nourrit d'un terreau fertile issu de la recherche fondamentale.

Marianne souhaite simplement être formée pour entrer sur le marché du travail avec une perspective de carrière intéressante en répondant aux exigences des organisations. Elle souhaite pouvoir traduire les connaissances intégrées lors de ses études supérieures en capacité productive pour les organisations. Elle pense que le point de rencontre entre la formation et les besoins des organisations constitue une clé de voûte indispensable pour concilier performance individuelle et organisationnelle. De plus, Marianne ne peut s'empêcher de penser que sa " valeur marchande " est garantie par l'université, ce qui la rend plus pertinente que ses homologues chinois. Elle considère contribuer à l'économie de son pays, sa compétitivité et ses innovations. Jean souligne que les IAE de France affichent des taux d'insertion professionnelle supérieurs à $80 \%$, mais l'indicateur ne semble pas retenir l'attention des 
décideurs. II propose ainsi de le retenir pour mettre en avant la nécessité d'investir pour l'État dans son système éducatif supérieur, en laissant de côté l'approche par les coûts pour retenir les gains futurs à l'échelle de la Nation.

\section{Conclusion}

L'université représente un atout majeur à l'échelle d'une nation pour trois raisons. Tout d'abord, l'université représente un lieu d'enseignement accessible, susceptible de former les esprits des nouvelles générations. Les citoyens deviennent capables de réfléchir, de modéliser, d'élaborer des stratégies, d'innover. Cette dynamique répond aux besoins des organisations qui pourront s'appuyer sur leurs collaborateurs pour créer, renforcer et maintenir leur avantage compétitif. La deuxième raison concerne tout particulièrement les sciences de gestion et du management, qui conduisent principalement des recherches appliquées, capables de répondre aux attentes des organisations et de proposer des leviers d'amélioration des pratiques. Des ponts continus existent ainsi entre les praticiens et les chercheurs afin d'offrir un cadre de réflexion structuré, capable d'appréhender des leviers d'efficacité et de compétitivité. La troisième raison concerne la nécessité pour la France d'exister sur la scène internationale en tant que Nation qui propose et apporte des modélisations, des cadres de référence pour mieux penser, et donc agir de manière efficace et pertinente.

La réussite de notre système universitaire repose sur trois conditions clés. D’une part, il est impératif de garantir l'accès à une formation supérieure de qualité, accessible à nos citoyens en termes financiers afin de former les nouvelles générations. Les jeunes étudiants méritent de trouver des métiers dont l'actif spécifique garantit leur avenir de façon durable. D’autre part, il est nécessaire d'œuvrer pour la valorisation et la reconnaissance des métiers des enseignants-chercheurs afin de ne pas décourager des vocations et de maintenir un bon niveau en recherche et en enseignement. Enfin, il est indispensable de laisser le temps aux enseignants-chercheurs d'exercer leur métier sans se faire happer par des tâches administratives qui peuvent être endossées par des professionnels dédiés à de telles activités. Ce temps disponible permet aux enseignants chercheurs de créer des espaces de réflexion permettant de créer des méthodologies d'approches en sciences de gestion et du management qui bénéficieront aux étudiants, garantissant ainsi la pérennité de leur emploi en convergence avec la rentabilité actionnariale.

Force est d'admettre que les entreprises françaises bénéficient de l'expertise des chercheurs, par l'intermédiaire des futurs salariés, mais également par la mise en œuvre de relations continues avec l'université. Investir dans l'université représente donc une garantie d'avantage compétitif sur du long terme pour la Nation française, ses citoyens et ses entreprises, et cela ne peut se limiter à un coût, une charge pour la société. II devient impératif de déterminer des indicateurs de performance adaptés à ce secteur d'activité spécifique que représente l'exception française qu'est l'université. 


\section{Conclusion générale}

Aude DevilLe

Le présent ouvrage collectif, porté par les 96 membres de la section 06 du CNU, élus et nommés, est publié à mi-parcours de la mandature 2019-2023. L'ensemble des listes et des sensibilités y sont représentées à travers 18 chapitres regroupés en cinq grandes thématiques. En complément de leur activité d'enseignant-chercheur souvent (lourdement) investi dans son institution, les membres de la section ont évalué, sur les premières sessions de la mandature, 942 dossiers de qualification aux fonctions de maître de conférences et de professeur des universités, 660 dossiers d'avancement de carrière et 304 dossiers de demande de PEDR. Nul doute qu'un tel investissement demande de l'engagement, du temps et une rigueur de tous les instants, tant dans le travail de préparation effectué par le bureau et les rapporteurs, qu'au moment de la tenue proprement dite des sessions. Chaque dossier est discuté par les rapporteurs qui en soulignent les faits marquants et observables. Des rapports de sessions sont ensuite produits, disponibles sur le site du CNU ; ils sont rédigés à l'attention des collègues pour leur donner les clés de réussite future ${ }^{1}$.

Les collègues qui siègent au sein de la section $06 \mathrm{du} \mathrm{CNU}$, comme pour toutes les autres sections, le font pour un mandat de quatre ans, avec un maximum de deux mandats consécutifs en tant que titulaire ${ }^{2}$. II s'agit de fait d'une instance mouvante, qui souhaite véritablement " incarner » la communauté académique des sciences de gestion et du management dans toute sa richesse de pensée. Dans le cadre de la mandature actuelle, le double objectif que se sont fixés les membres est de garantir l'intégrité et la promotion de notre discipline, une discipline certes jeune par rapport à d'autres (son " institutionnalisation » en France date des années 1970), mais une discipline qui a su démontrer son dynamisme et son indépendance, tant par ses objets, pratiques et méthodes de recherche que par le développement d'institutions et de formations spécifiques (le réseau IAE France en est une incarnation

1 [https://www.conseil-national-des-universites.fr/cnu/\#/entite/entiteName/CNU/idChild/31/ idNode/3696-3723], consulté le 21 septembre 2021.

2 [https://www.galaxie.enseignementsup-recherche.gouv.fr/ensup/CNU_elections_2019.htm], consulté le 21 septembre 2021. 
parmi d'autres), sans oublier la présence d'associations académiques au large rayonnement, y compris international, et soutenues activement par la FNEGE.

Les principes défendus par la section 06 du CNU s'inscrivent dans le respect absolu de règles éthiques et la reconnaissance de la diversité, mais aussi dans la prise en compte et la valorisation de l'ensemble des missions des enseignants-chercheurs. La section assume ainsi sa mission première d'évaluation disciplinaire et apporte des critères spécifiques et appropriés aux sciences de gestion et du management ; elle contribue à légitimer disciplinairement, d'un point de national, les compétences et expertises développées par les collègues dans leur quotidien de chercheur et d'enseignant engagé administrativement et/ou scientifiquement au sein d'instances nationales et/ou locales. Les contributions ici rassemblées livrent bien sûr l'écho de débats et controverses au sein de la section, faisant vivre et progresser une communauté qui cherche à relever les défis du monde actuel (politiques, économiques, sociologiques et écologiques). Mais ces débats et controverses sont conduits sans idéologie, uniquement avec conviction, dans un réel effort réflexif, et quelquefois critique, comme savent le cultiver tous les membres de la section 06 du CNU. 


\section{Bibliographie générale}

Abord de Chatillon E., Desmarais C. (2012), Le nouveau management public est-il pathogène ?, Management International, vol. 16, $n^{\circ} 3$, p. 10-24.

AcCARdo A., Corcuff P. (1986), La sociologie de Bourdieu : textes choisis et commentés, Bordeaux, Le Mascaret.

ACKER J., BARRY K., JOKE E. (1983), Objectivity and truth: problems in doing feminist research, Women's Studies International Forum, vol. 6, n 4, p. 423-435.

Acuna D., Allesina S., Kording K. (2012), Predicting scientific success, Nature, vol. 489, p. 201-202.

AdAMS J. (1965), Inequity in social exchange, Advances in Experimental Social Psychology, vol. 2, p. 267-299.

AdAMs G., BAlfour D., ReEd G. (2006), Abu Ghraib, administrative evil, and moral inversion: the value of "putting cruelty first", Public Administration Review, vol. 66, n०5, p. 680-693.

AdLeR P., AdLER P. (1993), Ethical issues in self-censorship: ethnographic research on sensitive topics, in RENZETTI C., LEE R. (dir.), Researching sensitive topics, Thousand Oaks (CA), Sage, p. 249-266.

Adobor H., DANeShFAR A. (2006), Management simulations: determining their effectiveness, Journal of Management Development, vol. 25, n², p. 151-168.

AgERON B., BENZIDIA S., BourLAKIS M. (2018), Healthcare logistics and supply chain: issues and future challenges, Supply Chain Forum: An International Journal, vol. 19, n 1, p. 1-3.

Ageron B., Bentahar O., Gunasekaran A. (2020), Digital supply chain: challenges and future directions, Supply Chain Forum: An International Journal, vol. $21, \mathrm{n}^{\circ} 3$, p. 133-138.

AGGERI F. (2017), How can performativity contribute to management and organization research? Theoretical perspectives and analytical framework, M@n@gement, vol. 20, n 1, p. 28-69.

AgIER M. (1997), Ni trop près, ni trop loin : de l'implication ethnographique à l'engagement intellectuel, Gradhiva, $\mathrm{n}^{\circ} 21$, p. 69-76.

Aguinis H., Cummings C., Ramani R., Cummings T. (2020), An A is an A: the new bottom line for valuing academic research, Academy of Management Perspectives, vol. 34, n 1 , p. 135-154. 
AIT-TALEB N., MANI Z. (2019), Les facteurs et les formes de résistance des apprenants à une innovation pédagogique : le cas d'un business simulation game, Management \& Avenir, $\mathrm{n}^{\circ} 111$, p. 13-34.

Albouy M. (2019), Business schools : en route pour l'uberisation des enseignants-chercheurs ?, The Conversation, 26 août.

ALEXANDER S., PILLAY R., SMITH B. (2018), A systematic review of the experiences of vulnerable people participating in research on sensitive topics, International Journal of Nursing Studies, vol. 88, p. 85-96.

ALTER N. (2009), Donner et prendre : la coopération en entreprise, Paris, La Découverte.

Alvarez J., Djaouti D. (2012), Introduction aux serious games, Paris, Éditions Questions Théoriques, $2^{\mathrm{e}}$ éd.

AnNoot E. (2011), Le métier d'enseignant chercheur, in IMELDA E., MuRPhy M., PAYeur A., Duval R. (dir.), Mutations de l'enseignement supérieur et internationalisation, Bruxelles, De Boeck, p. 221-231.

Ansoff H. (1965), Corporate strategy, New York, McGraw-Hill.

Astley W., ZAmmuto R. (1992), Organization science, managers, and language games, Organization Science, vol. 3, n 4, p. 443-460.

Aubert N. (2016), La participation des salariés : une expression du don dans les organisations, Revue Interdisciplinaire Management, Homme \& Entreprise, $\mathrm{n}^{\circ} 21$, p. 69-90.

AUDRIN C. (2020), Les émotions dans la formation enseignante : une perspective historique, Recherches en Éducation, $\mathrm{n}^{\circ} 41$, p. 5-19.

Aurifeille J.-M., Colin J., FabBe-Costes N., Jaffeux C., PAChé G. (1997), Management logistique : une approche transversale, Paris, Litec.

AUSTIN J. (1991), Quand dire, c'est faire, Paris, Le Seuil.

AWAN S., Bel G., ESTEVE M. (2020), The benefits of PSM: an oasis or a mirage?, Journal of Public Administration Research \& Theory, vol. 30, n 4, p. 619-635.

AYIMPAM S. (2015), Enquêter sur la violence : les défis méthodologiques et émotionnelsCivilisations, $\mathrm{n}^{\circ}$ 64, p. 57-66.

AyıMPAM S., BouJu J. (2015), Objets tabous, sujets sensibles, lieux dangereux : les terrains difficiles aujourd'hui, Civilisations, $n^{\circ} 64$, p. 11-20.

BAKKeR A., DEMEROUtI E. (2007), The job demands-resources model: state of the art, Journal of Managerial Psychology, vol. 22, n³, p. 309-328.

BALECH S., BenAVENT C. (2019), Les techniques du NLP pour la recherche en sciences de gestion, Papier de recherche non publié, Université Paris Nanterre.

BANDURA A. (2000), Moral disengagement in the perpetration of inhumanities, in Miller A. (dir.), Perspectives on evil and violence, Londres, Psychology Press, p. 193-209.

Barel Y., Frémeaux S. (2008), Le don gratuit : le cas d'un établissement public, Gérer et Comprendre, n94, p. 80-89.

BARET C., HuAUlt I., PICQ T. (2006), Management et réseaux sociaux : jeux d'ombres et de lumières sur les organisations, Revue française de gestion, $\mathrm{n}^{\circ}$ 163, p. 93-106.

BARTH I., Noguera F. (2018), L'utilité des sciences de gestion pour la société, in Peretti, J.-M., Plane J.-M., Scouarnec A., Thévenet M. (dir.), Une vision des ressources 
humaines sans frontières : mélanges en l'honneur de Jacques Igalens, Caen, Éditions Management \& Société, p. 365-370.

Baudet C., Kalika M., Mottet V., Favre F. (2016), Sciences de gestion : des managers, des chercheurs... ou les deux ?, The Conversation, 7 juin.

Baudet-Michel S., BerRoir S., Grasland C., Guérois M., Madelin M., Maisonobe M., PIStre P., Ronchall J., Zanin C. (2020), Vers une désertification scientifique et universitaire du territoire français?, Document de travail, Université Paris 1 Panthéon-Sorbonne.

BeAud O., VAtin F. (2020), La profession universitaire : une expression malheureuse, "enseignant-chercheur ", et un statut dégradé (I), Commentaire, n 172 , p. 859-870.

BEAUd O., VATIN F. (2021), La profession universitaire : une expression malheureuse, "enseignant-chercheur ", et un statut dégradé (II), Commentaire, n 173 , p. 141-152.

BEAULIEU M., BENTAHAR O. (2021), Digitalization of the healthcare supply chain: a roadmap to generate benefits and effectively support healthcare delivery, Technological Forecasting \& Social Change, vol. 167, Article 120717.

BeAulieu M., Bentahar O., Benzidia S. (2020), The evolution of healthcare logistics: the Canadian experience, Journal of Applied Business \& Economics, vol. 22, $\mathrm{n}^{\circ} 14$, p. 196-202.

Beaulieu M., Roy J., LANdRY S., Michaud M., Roy C. (2014), La logistique hospitalière au Québec : passé, présent et futur, Gestion, vol. 39, n³, p. 56-62.

BÉCHARd J.-P., Pelletier P. (2000), Comprendre les innovations pédagogiques en milieu universitaire : une exploration en sciences de la gestion, Cahier de Recherche OIPG n²000-002, HEC Montréal.

BÉJI-BÉCHEUR A., HeRBERT M., OzÇAĞLAR-Toulouse N. (2011), Étudier l'ethnique : la construction de la responsabilité des chercheurs face à un sujet sensible, Revue française de gestion, $n^{\circ} 216$, p. 111-128.

BensebaA F., Vandangeon-Derumez I. (2008), L'université qui change : un oxymore ?, Actes de la XVII Conférence de l'Association Internationale de Management Stratégique, Nice, p. 1-26 (CD-rom).

BentahAR O., BenzidiA S., dir. (2019), Supply chain management de la santé, Caen, Éditions Management \& Société.

Benzidia S., MAKaOUI N., Bentahar O. (2021), The impact of big data analytics and artificial intelligence on green supply chain process integration and hospital environmental performance, Technological Forecasting \& Social Change, vol. 165, Article 120557.

BERGER J.-L., D'AsCOLI Y. (2011), Les motivations à devenir enseignant : revue de la question chez les enseignants de première et deuxième carrière, Revue française de pédagogie, $\mathrm{n}^{\circ} 175$, p. 113-146.

BESSIRE D., FABRE P. (2014), New public management et évaluation de la recherche publique : I'invention du " produisant ", Finance Contrôle Stratégie, vol. 17, $n^{\circ} 4$, p. 31-58.

BEZES P. (2005), Le renouveau du contrôle des bureaucraties : l'impact du new public management, Informations Sociales, $\mathrm{n}^{\circ} 126$, p. 26-37. 
Biddle L., Cooper J., Owen-Smith A., Klineberg E., Bennewith O., Hawton K., KAPUR N., Donovan J., GunNell D. (2013), Qualitative interviewing with vulnerable populations: individuals' experiences of participating in suicide and self-harm based research, Journal of Affective Disorders, vol. 145, n³, p. 356-362.

BIGGS J., TANG C. (2011), Train-the-trainers: implementing outcomes-based teaching and learning in Malaysian higher education, Malaysian Journal of Learning \& Instruction, vol. 8, p. 1-19.

BLoom B. (1956), Taxonomy of educational objectives. Volume 1: Cognitive domain, New York, McKay.

Bonenfant M., Philippette T. (2018), Rhétorique de l'engagement ludique dans des dispositifs de ludification, Sciences du Jeu, vol. 10, p. 1-16.

BORNMANN L. (2013), What is societal impact of research and how can it be assessed? A literature survey, Journal of the American Society for Information Science \& Technology, vol. 64, n², p. 217-233.

BORRY E. (2017), Ethical climate and rule bending: how organizational norms contribute to unintended rule consequences, Public Administration, vol. 95, $\mathrm{n}^{\circ} 1$, p. 78-96.

BouILLON F., MARION F., TALLIO V. (2005), Terrains sensibles : expériences actuelles de l'anthropologie, Paris, Éditions de l'EHESS.

Boulet E., HaRARI-Kermadec H. (2014), Le rôle de la quantification dans le processus de marchandisation de l'université : étude de cas d'une université anglaise, Politiques \& Management Public, vol. 31, n 4, p. 405-420.

Boumaza M., CAMPANA A. (2007), Enquêter en milieu « difficile », Revue française de science politique, vol. 57, $\mathrm{n}^{\circ} 1$, p. 5-25.

BouRdieu P. (1980), Le sens pratique, Paris, Éditions de Minuit.

BouRdieu P. (1984), Homo academicus, Paris, Éditions de Minuit.

BouRdieu P. (1989), La noblesse d'État : grandes écoles et esprit de corps, Paris, Éditions de Minuit.

BOURDIEU P. (2007), La misère du monde, Paris, Le Seuil.

BousLIKHANE A. (2011), Enseignement de l'entrepreneuriat : pour un regard paradigmatique autour du processus entrepreneurial, Thèse de doctorat en Sciences de Gestion, Université de Nancy 2.

BRILlet F., SCOUARNEC A., dir. (2020), Penser et agir compétences : un changement de paradigme pour les établissements et les formations, Paris, FNEGE.

BRINN T., Jones M., PendlebuRy M. (2000), Measuring research quality: peer review 1 , citation indices 0 , Omega, vol. 28, n², p. 237-239.

BRoedker C., Chong K.-M., Mouritsen J. (2020), The counter-performativity of calculative practices: mobilising rankings of intellectual capital, Critical Perspectives on Accounting, vol. 72, article 102100.

BRUNI L. (2010), Pour une théorie de la réciprocité, plurielle et pluraliste, Revue du MAUSS, $\mathrm{n}^{\circ} 35$, p. 221-245.

BRUNSSON N. (1993), The irrational organization: irrationality as a basis for organizational action and change, New York, John Wiley \& Sons.

BRUNSSON N. (2002), The organization of hypocrisy: talk, decisions and actions in organizations, Copenhague, Copenhagen Business School Press. 
Burke P., Stets J. (2009), Identity theory, Oxford, Oxford University Press.

BURLAUd A., COLASSE B. (2010), Normalisation comptable internationale : le retour du politique ?, Comptabilité Contrôle Audit, vol. 16, n³, p 153-175.

Caillé A., Chanial P., dir. (2009), L'université en crise : mort ou résurrection ?, Revue du MAUSS, $\mathrm{n}^{\circ} 33$, p. 5-30.

CAILlé A., GRÉsy J.-E. (2014), La révolution du don : le management repensé à la lumière de l'anthropologie, Paris, Le Seuil.

CALLon M. (2010), Performativity, misfires and politics, Journal of Cultural Economy, vol. 3, n², p. 163-169.

CAMERON J., BANKo K., PIERCE W. (2001), Pervasive negative effects of rewards on intrinsic motivation: the myth continues, The Behavior Analyst, vol. 24, n 1, p. 1-44.

CAPPELLETTI L. (2009), La recherche-intervention : une réponse au besoin d'evidence-based management en contrôle de gestion ?, Actes du Congrès de l'Association Francophone de Comptabilité sur la Place de la Dimension Européenne dans la Comptabilité Contrôle Audit, Strasbourg, p. 1-24 (CD-rom).

CARTON G., DAMERon S. (2012), Proposition d'un cadre d'analyse des modes de diffusion de connaissances en management, Actes de la XXI Conférence de l'Association Internationale de Management Stratégique, Lille, p. 1-18 (CD-rom).

CARTON G., Mouricou P. (2017), À quoi sert la recherche en management ? Une analyse systématique de la littérature anglo-saxonne sur le débat rigueur-pertinence (1994-2013), M@n@gement, vol. 20, n², p. 166-203.

CASCIOLI F. (2020), La performativité du MOOC sur les représentations de l'apprenant : le cas du parcours MOOC, Thèse de doctorat en Sciences de Gestion, HESAM Paris.

Cavory G., Gardet E., Thevenard-Puthod C. (2019), OGP'Lay : un dispositif permettant de stimuler les compétences des étudiants, Actes du Colloque sur Questions de Pédagogies dans l'Enseignement Supérieur, Brest, p. 1-13.

CAzAl D., Davoine E., Louart P., CheValier F. (2010), GRH et internationalisation : nouveaux contextes, nouveaux enjeux, Paris, Vuibert.

CERDIN J.-L. (2007), S'expatrier en toute connaissance de cause, Paris, Eyrolles.

CeKerevac Z. (2013), Key performance indicators and dashboards for transportation and logistics, Transport, vol. 11, n³, p. 43-50.

CHANDLER A. (1962), Strategy and structure: chapters in the history of the American industrial enterprise, Cambridge (MA), MIT Press.

ChANNELS N. (1993). Anticipating media coverage: methodological decisions in criminal justice research, in RENZETTI C., LEE R. (dir.), Researching sensitive topics, Thousand Oaks (CA), Sage, p. 267-280.

CHANUT V., Rojot J. (2011), Réinventer l'évaluation : les nouvelles règles du jeu RH dans une administration publique, Management \& Avenir, $n^{\circ} 49$, p. 195-222.

CHARLE C. (2009), L'évaluation des enseignants-chercheurs : critiques et propositions, Vingtième Siècle : Revue d'Histoire, $\mathrm{n}^{\circ} 102$, p. 159-170.

CharReaux G., dir. (1997), Le gouvernement des entreprises. Corporate governance : théories et faits, Paris, Economica.

Charreaux G. (2018), Préface, in Burkhardt K., Desbrières P. (dir.), Cas en gouvernance des organisations, Caen, Éditions Management \& Société, p. 5-7. 
Charreaux G., Gervais M. (2007), La « piste aux étoiles » : un commentaire sur le dernier classement des revues élaboré par la section 37 du CNRS, Finance Contrôle Stratégie, vol. 10, $\mathrm{n}^{\circ} 4$, p. 5-16.

Chatelain-Ponroy S., Deville A. (2020), Crise sanitaire et technocratie : il existe DES modes de contrôle !, Revue française de gestion, vol. 46, n²93, p. 197-204.

CHEN B., BUtTE A. (2016), Leveraging big data to transform target selection and drug discovery, Clinical Pharmacology \& Therapeutics, vol. 99, n³, p. 285-297.

CHOllet A. (2019), Différence entre jeu vidéo, serious game, serious-gaming et ludification : proposition d'une typologie des usages managériaux des technologies et pratiques ludiques, Actes de la XXIVe Conférence de l'Association Information \& Management, Nantes, p. 1-15 (CD-rom).

CHRISTOPHER M. (2016), Logistics and supply chain management, Harlow, Pearson Education, $5^{\text {e éd. }}$

Cieply S., GRAND I. (2019), Quels usages pour les Open Badges dans l'enseignement supérieur? Analyse de la diffusion d'une innovation à I'IAE Caen, Management \& Avenir, $\mathrm{n}^{\circ} 113$, p. 15-38.

Clerkin R., PAynter S., TAYlor J. (2009), Public service motivation in undergraduate giving and volunteering decisions, American Review of Public Administration, vol. $39, n^{\circ} 6$, p. 675-698.

CLOт Y. (2010), Le travail à cœur : pour en finir avec les risques psychosociaux, Paris, La Découverte.

Colin J. (2005), Le supply chain management existe-t-il réellement ?, Revue française de gestion, $\mathrm{n}^{\circ} 156, \mathrm{p} .135-149$.

CollaRd D. (2018), Le travail, au-delà de l'évaluation : normes et résistances, Toulouse, Éditions Eres.

ColquitT J. (2001), On the dimensionality of organizational justice: a construct validation of a measure, Journal of Applied Psychology, vol. 86, n³, p. 386-400.

Côme T., Rouet G. (2016), Comment évaluer les universitaires? Efficacité et performance des structures et dispositifs, Gestion \& Management Public, vol. 5, $\mathrm{n}^{\circ} 1$, p. 61-85.

CôME T., ROUET G. (2017), Statut des personnels, gouvernance et justice organisationnelle : le cas des universités françaises, Revue Interdisciplinaire Management, Homme \& Entreprise, $n^{\circ} 28$, p. 77-89.

CONdomines B., Hennequin E. (2013), Étudier les sujets sensibles : les apports d'une approche mixte, Revue Interdisciplinaire Management, Homme \& Entreprise, $\mathrm{n}^{\circ} 5$, p. 12-27.

CORROCHER N., FontANA R. (2008), Objectives, obstacles and drivers of ICT adoption: what do IT managers perceive?, Information Economics \& Policy, vol. 20, $n^{\circ} 3$, p. 229-242.

COURPASSON D., GUEDRI Z. (2007), Les professeurs-chercheurs en management face à la recherche scientifique, Revue française de gestion, $\mathrm{n}^{\circ}$ 178-179, p. 173-194.

Cox A., IREland P., Lonsdale C., SAnderson J., Watson G. (2001), Supply chains, markets and power: managing buyer and supplier power regimes, Londres, Routledge. 
CreWson P. (1997), Public-service motivation: building empirical evidence of incidence and effect, Journal of Public Administration Research \& Theory, vol. 7, $\mathrm{n}^{\circ} 4$, p. 499-518.

Cropanzano R., Folger R. (1989), Referent cognitions and task decision autonomy: beyond equity theory, Journal of Applied Psychology, vol. 74, n² 2, p. 293-299.

Crozier M., Friedberg E. (1977), L'acteur et le système : les contraintes de l'action collective, Paris, Le Seuil.

CSIKSZENTMIHALYI M. (1990), Flow: the psychology of optimal experience, New York, Harper \& Row.

DAVID A. (2004), Les connaissances en sciences de gestion : devons-nous choisir entre scientificité et actionnabilité ?, in SAVAll H., Bonnet M., Peron M. (dir.), Traversée des frontières entre méthodes de recherche qualitatives et quantitatives, Lyon, ISEOR, p. 851-876.

Demetrovics Z., Griffiths M. (2012), Behavioral addictions: past, present and future, Journal of Behavioral Addictions, vol. 1, n 1, p. 1-2.

De MonteCleR M.-C. (2020), La fronde contre la loi de programmation de la recherche s'amplifie dans les universités, Dalloz Actualité, 19 novembre.

DENIS J.-P. (2010), De l'autonomie des universités à l'indépendance des universitaires : retour vers le futur, Journal du MAUSS, 24 septembre.

DENIS J.-P. (2014), Introduction au hip-hop management, Caen, Éditions Management \& Société.

DENIS J.-P. (2015), La recherche française en management pèse-t-elle - enfin ! dans I' " academic game »?, The Conversation, 5 octobre.

DENIS J.-P. (2018), De crier mon cœur de chercheur en management stratégique a commencé, Revue Internationale de Psychosociologie et de Gestion des Comportements Organisationnels, $n^{\circ} 57$, p. 231-262.

DENIS J.-P. (2019), Procès France Télécom : « Faut-il pénaliser l'incompétence managériale ? ", Le Monde, 7 juin.

DeNIS J.-L., LeHOUX P., TRÉ G. (2009), L'utilisation des connaissances produites, in RIDDE V., DAGENAIS C. (dir.), Approches et pratiques en évaluation de programme, Montréal, Presses de l'Université de Montréal, p. 177-191.

DeRMARKAR S., GendRON Y. (2019), The incursion of neoliberalism into audit standard-setting arenas: a disproportionate influence?, Comptabilité Contrôle Audit, vol. $25, n^{\circ} 2$, p. 55-85.

Descombes V. (2009), L'identité collective d'un corps enseignant, Revue du MAUSS, $\mathrm{n}^{\circ} 33$, p. 343-362.

DESROSIÈRES A. (1993), La politique des grands nombres : histoire de la raison statistique, Paris, La Découverte.

DGRH (2021a), Les mesures d'accompagnement mises en œuvre par les établissements dans le cadre des campagnes de suivi de carrière des enseignants-chercheurs 2017-2019, Document de travail, Paris, mars.

DGRH (2021b), Le suivi de carrière, Note de Recherche, $\mathrm{n}^{\circ} 2$, février.

DickSON-SWIFT V., JAMES E., KIPPEN S., LIAMPUTTONG P. (2006), Blurring boundaries in qualitative health research on sensitive topics, Qualitative Health Research, vol. $16, n^{\circ} 6$, p. 853-871. 
DickSON-SWIFT V., JAMES E., KIPPEN S., LIAMPUTTONG P. (2008), Risk to researchers in qualitative research on sensitive topics: issues and strategies, Qualitative Health Research, vol. 18, n²1, p. 133-144.

DiefENBACH T. (2009), New public management in public sector organizations: the dark sides of managerialistic "enlightenment", Public Administration, vol. 87, $\mathrm{n}^{\circ} 4$, p. 892-909.

DRUCKER P. (1967), The effective executive, New York, Harper \& Row.

DUBET F. (2020), Le métier d'enseignant : une alchimie à reconstruire, Administration \& Éducation, $\mathrm{n}^{\circ} 168$, p. 13-20.

Dubey R., GunASEKARAN A., CHILDE S. (2019), Big data analytics capability in supply chain agility: the moderating effect of organizational flexibility, Management Decision, vol. 57, n8, p. 2092-2112.

Ducroca C., Gervals M. (2013), Le coût complet par diplôme à l'université : quel apport vu les problèmes de mesure ?, Finance Contrôle Stratégie, vol. 16, $\mathrm{n}^{\circ} 4$, p. 1-29.

Duforez A. (1995), Complexité des fusions-acquisitions, Thèse de doctorat en Sciences de Gestion, Université Paris 1 Panthéon-Sorbonne.

Dulbecco P., Beer M.-C., Delpech de Saint-Guilhem J., Dubourg-Lavroff S., PIMMEL E. (2018), Les innovations pédagogiques numériques et la transformation des établissements d'enseignement supérieur, Rapport n²018-049, Paris, Inspection générale de l'Administration, de l'Éducation nationale et de la Recherche.

DUMEZ H. (2015), De nouvelles perspectives sur la performativité, Gérer et Comprendre, n०119, p. 82-83.

DuMEZ H. (2016), L’hypocrisie organisationnelle, in SAussols J.-M. (dir.), Les organisations : état des savoirs, Auxerre, Éditions Sciences Humaines, p. 267-273.

DUPUY Y., LACROIX M., NARO G. (2006), Identités et dilemmes de l'enseignant-chercheur en sciences comptables, Comptabilité Contrôle Audit, vol. 12, n², p. 9-26.

DURAND T., DAMERON S. (2011), Where have all the business schools gone?, British Journal of Management, vol. 22, n³, p. 559-563.

Duтот V. (2021), Un bon chercheur c'est quoi ? Un mauvais chercheur c'est quoi ? Management \& Data Science, vol. 5, n5 Article 17118.

ESPELAND W., SAUDER M. (2007), Rankings and reactivity: how public measures recreate social worlds, American Journal of Sociology, vol. 113, n² 1, p. 1-40.

ESPELAND W., StEVENS M. (2008), A sociology of quantification, European Journal of Sociology, vol. 49, $\mathrm{n}^{\circ} 3$, p. 401-436.

ESTRYN-BEHAR M. (2008), Santé et satisfaction des soignants au travail en France et en Europe, Rennes, Presses de l'EHESP.

EWANGo-ChATELET A. (2017), Les dynamiques intrapreneuriales des innovations pédagogiques à l'Université : une approche contextuelle, Thèse de doctorat en Sciences de Gestion, Université Paris Saclay.

FABBE-Costes N. (2000), Le rôle transformatif des SIC et TIC sur les interfaces multi-acteurs de la distribution et de la logistique, in FABBE-COSTES N., Colin J., PACHÉ G. (dir.), Faire de la recherche en logistique et distribution, Paris, VuibertFnege, p. 171-194. 
FABbe-Costes N., SÉPARI S. (2020), Covid-19, une remise en question des fondamentaux de la logistique et du SCM ?, Revue française de gestion, ${ }^{\circ} 293$, p. 205-217.

FAbBe-Costes N., Fulconis F., Koulikoff-SOuviRon M. (2013), La recherche en management logistique : la question des unités d'analyse et du périmètre, in FABBECOSTES N., PACHÉ G. (dir.), La logistique : une approche innovante des organisations, Aix-en-Provence, Presses Universitaires de Provence, p. 49-60.

FARJAUDON A.-L., MORALES J. (2013), In search of consensus: the role of accounting in the definition and reproduction of dominant interests, Critical Perspectives on Accounting, vol. 24, $\mathrm{n}^{\circ} 2$, p. $154-171$.

FARRELL C. (2005), Perceived effectiveness of simulations in international business pedagogy, Journal of Teaching in International Business, vol. 16, n³, p. 71-88.

FAURÉ B., Gramaccia G. (2006), La pragmatique des chiffres dans les organisations : de l'acte de langage à l'acte de calcul, Études de Communication, n’ 29, p. 25-37.

Fauré B., Brummans B., Giroux H., TAYlor J. (2010), The calculation of business, or the business of calculation? Accounting as organizing through everyday communication, Human Relations, vol. 63, n 8 , p. 1249-1273.

Fayolle A., Verzat C. (2009), Pédagogies actives et entrepreneuriat : quelle place dans nos enseignements?, Revue de l'Entrepreneuriat, vol. 2, n² 2, p. 1-15.

FERNANDEZ F. (2005), L'engagement émotionnel durant l'enquête sociologique : retour sur une observation " anonyme " auprès d'ex-usagers de drogues, Carnets de Bord en Sciences Humaines, n॰ 9, p. 78-87.

FISHER G., AGUINIS H. (2017), Using theory elaboration to make theoretical advancements, Organizational Research Methods, vol. 20, n³, p. 438-464.

Folger R., KONOVSKY M. (1989), Effects of procedural and distributive justice on reactions to pay raise decisions, Academy of Management Journal, vol. $32, \mathrm{n}^{\circ} 1$, p. $115-130$.

FRANCESCHINI F., MAISANO D. (2011), Criticism on the hg-index, Scientometrics, vol. 86, n' 2 , p. 339-346.

FrémeAuX S., Michelson G. (2011), "No strings attached": welcoming the existential gift in business, Journal of Business Ethics, vol. 99, n 1, p. 63-75.

Frémeaux S., Grevin A., MAsclef O. (2014), Les théories du don en sciences sociales, Cahiers de Recherche du GRACE, série Don, Échange et Gratuité, nº1-2014, Paris.

French J., Raven B. (1959), The bases of social power, in Cartwright D. (dir.), Studies in social power, Ann Arbor (MI), University of Michigan Press, p. 150-167.

FulCONIS F. (1999), Logistique et administration générale des structures en réseau, Gestion 2000, vol. 16, n4, p. 109-125.

FULCONIS F., KACIOUI-MAURIN E. (2013), Apports de l'ambidextrie organisationnelle au " management logistique » : études de cas dans l'industrie de la prestation logistique, Logistique \& Management, vol. 21, n² 2 p. 19-32.

FULCONIS F., PACHÉ G. (2011), Entre innovation et optimisation : la décision en logistique à la croisée des chemins, Management \& Avenir, n 48, p. 158-178.

FulCONIS F., PACHÉ G. (2020), Pandémie de Covid-19 et chaînes logistiques : une mondialisation désenchantée, Revue française de gestion, n²93, p. 171-181. 
FulConis F., PACHÉ G., ReYNAUd E. (2016), Vers une nouvelle forme de croissance économique : les apports des recherches en logistique et supply chain management, Revue française de gestion, $\mathrm{n}^{\circ} 261, \mathrm{p} .127-149$.

Fulconis F., MeUrier B., PACHÉ G. (2019), Capacités dynamiques, réseaux et chaînes logistiques, Revue Internationale des Sciences de l'Organisation, $\mathrm{n}^{\circ} 7$, p. 43-78.

GABELLIERI E. (2001), Un don sans retour? Pour une analogie et une " catalogie » du don, in Dumont J.-N. (dir.), Le don : théologie, philosophie, psychologie, sociologie, Lyon, Le Collège Supérieur, p. 75-83.

Gabellieri E. (2014), Équivocité ou analogie du don ? Le don des philosophes, le don des sociologues et le don de soi dans la vraie vie, Cahiers de Recherche du GRACE, série Don, Échange et Gratuité, n 7-2014, Paris.

GALVEZ-BEHARD G. (2010), Les revues de sciences humaines et sociales face à l'évaluation, Le Mouvement Social, n²33, p. 159-164.

GARçon F. (2012), Le dernier verrou : en finir avec le Conseil National des Universités, Paris, The Media Faculty.

GarReAu L., DE Montmorillon B. (2019), La grande transformation du métier de chercheur : discussion et points de vue, M@n@gement, vol. 22, n4, p. 725-738.

GATIGNOL C. (2014), L'environnement professionnel des enseignants-chercheurs français explique-t-il leurs parcours de carrière?, @GRH, $\mathrm{n}^{\circ} 11, \mathrm{p} .51-80$.

GeBREITER F. (2022), A profession in peril? University corporatization, performance measurement and the sustainability of accounting academia, Critical Perspectives on Accounting, Article 102292, à paraître.

Georgescu I., Grima, F., BARRedA M. (2018), From the dark side of public sector motivation to the white side of unethical pro-organizational behavior: the case of upcoding in a public sector hospital, Proceedings of the 19th EURAM Congress, Reykjavik, p. 1-15 (CD-rom).

GER G., SANDIKCI O. (2006), Doing research on sensitive topics: studying covered Turkish women, in BELK R. (dir.), Handbook of qualitative research methods in marketing, Cheltenham, Edward Elgar, p. 509-520.

Ghozlane S., Deville A., Dumez H. (2016), Enseignement supérieur : mythes et réalités de la révolution digitale, Gérer et Comprendre, n 126, p. 28-38.

GIANNAKIS M., SPANAKI K., DUBEY R. (2019), A cloud-based supply chain management system: effects on supply chain responsiveness, Journal of Enterprise Information Management, vol. 32, $n^{\circ} 4$, p. 585-607.

GIAUQUE D. (2003), New public management and organizational regulation: the liberal bureaucracy, International Review of Administrative Sciences, vol. 69, $\mathrm{n}^{\circ} 4$, p. 567-592.

Giauque D., Ritz A., Varone F., Anderfuhren-Biget S. (2012), Resigned but satisfied: the negative impact of public service motivation and red-tape on work satisfaction, Public Administration, vol. 90, n¹, p. 175-193.

Gillet P., Zimnovitch H. (2011), La responsabilité de l'enseignant-chercheur dans la formation des ingénieurs financiers, Revue française de gestion, n²16, p. 93-109.

GINGRAS Y. (2008), La fièvre de l'évaluation de la recherche du mauvais usage de faux indicateurs, Bulletin de Méthodologie Sociologique, vol. 100, n 1, p. 41-44. 
GINGRAS Y. (2014), Les dérives de l'évaluation de la recherche: du bon usage de la bibliométrie, Paris, Éditions Raisons d'Agir.

Giraud L., Hernandez S., Autissier D., McGonigal A. (2021), L'évolution des compétences managériales face à l'essor de l'intelligence artificielle : une approche par les méthodes mixtes, Management \& Avenir, n²122, p. 143-169.

Gomez P.-Y., GRevin A., MASClef O., dir. (2015), L'entreprise, une affaire de don : ce que révèlent les sciences de gestion, Bruyères-le-Châtel, Éditions Nouvelle Cité.

Goujon Belghit A., Husser J. (2021), Quels critères d'évaluation de la responsabilité sociétale des enseignants-chercheurs en management?, Management \& Sciences Sociales, $n^{\circ} 30$, p. 38-50.

GRANT A. (2008), Does intrinsic motivation fuel the prosocial fire? Motivational synergy in predicting persistence, performance, and productivity, Journal of Applied Psychology, vol. 93, n¹, p. 48-58.

GREenBerg J. (1987), A taxonomy of organizational justice theories, Academy of Management Review, vol. 12, $n^{\circ} 1$, p. 9-22.

GREENWOOD D., LEVIN M. (2007), Introduction to action research: social research of social change, Thousand Oaks (CA), Sage, $2^{\mathrm{e}}$ éd.

Greselle-ZaïBet O., Kleber A., Dejoux C. (2018), Le hackathon en mode design thinking ou quelles modalités pour former à des compétences méthodologiques et comportementales?, Management \& Avenir, $\mathrm{n}^{\circ} 104$, p. 149-171.

GREVIN A. (2013), Pour un réalisme de la recherche et du management : reconnaître le don au cœur du travail, Économies \& Sociétés, série Économie de l'Entreprise, $\mathrm{n}^{\circ} 22$, p. 33-62.

Guerrero-Bote P., Moya-Anegón F. (2012), A further step forward in measuring journals' scientific prestige: the SJR2 indicator, Journal of Informetrics, vol. $6, \mathrm{n}^{\circ} 4$, p. 674-688.

GUILLAUME H., MACRON E. (2007), Enseignement supérieur, recherche, innovation : quels acteurs?, Esprit, ${ }^{\circ} 12$, p. 160-187.

GuILLON B. (2016), L'évaluation des enseignants-chercheurs via les seules revues scientifiques classées : des aspects contre-productifs pérennisés, Management \& Sciences Sociales, $\mathrm{n}^{\circ} 21$, p. 78-89.

HASPESLAGH P., JEMISON D. (1991), Managing acquisitions: creating value through corporate renew, New York, Simon \& Schuster.

Hatchuel A. (1994), Les savoirs de l'intervention en entreprise, Entreprise \& Histoire, $\mathrm{n}^{\circ}$ 7, p. 59-75.

HATCHUEl A. (2019), Exit to the past and voice for the future : sciences de gestion, sciences fondamentales de l'action collective, Revue française de gestion, $\mathrm{n}^{\circ} 285$, p. 43-57.

HAYEK F. (1945), The use of knowledge in society, American Economic Review, vol. 35, n4, p. 519-530.

HÉNAFF M. (2012), Le don des philosophes : repenser la réciprocité, Paris, Le Seuil.

HENNEQUin E., dir. (2012), La recherche à l'épreuve des terrains sensibles: approches en sciences sociales, Paris, L'Harmattan.

HINES R. (1988), Financial accounting: in communicating reality, we construct reality, Accounting, Organizations \& Society, vol. 13, n³, p. 251-261. 
HIRSCHMAN A. (2014), Les passions et les intérêts, Paris, Presses Universitaires de France.

Hood C. (1991), A public management for all seasons?, Public Administration, vol. $69, \mathrm{n}^{\circ} 1$, p. 3-19.

Hopwood A. (2008), Changing pressures on the research process: on trying to research in an age when curiosity is not enough, European Accounting Review, vol. 17, n¹, p. 87-96.

Houston D. (2006), "Walking the walk" of public service motivation: public employees and charitable gifts of time, blood, and money, Journal of Public Administration Research \& Theory, vol. 16, nº 1, p. 67-86.

HUCHETTE C. (2020), La conduite d'un processus d'intégration organisationnelle depuis les réunions de direction : le cas d'une fusion en milieu universitaire, Thèse de DBA, Université Paris Dauphine.

Husson J. (2009), L'hôpital en quête de performance : perspectives méthodologiques pour un nouveau risk-management hospitalier, Gestion 2000, vol. 26, n 5 , p. $65-81$.

IDE P., de Peyrelongue B., Grevin A., Moneyron J.-D. (2021), Recevoir pour donner : relancer la dynamique du don dans l'entreprise, Bruyères-le-Châtel, Éditions Nouvelle Cité.

Imelda E., Murphy M., Payeur A., Duval R., dir. (2011), Mutations de l'enseignement supérieur et internationalisation, Bruxelles, De Boeck.

INSEL A. (2009), Publish or perish ! La soumission formelle de la connaissance au capital, Revue du MAUSS, n³3, p. 141-153.

JAMES C., ROFFE I. (2000), The evaluation of goal and goal-free training innovation, Journal of European Industrial Training, vol. 24, n 1, p. 12-20.

Jensen U., Andersen L., Holten A. (2019), Explaining a dark side: public service motivation, presenteeism, and absenteeism, Review of Public Personnel Administration, vol. 39, $\mathrm{n}^{\circ}$ 4, p. 487-510.

JUVEN P.-A., PIERRU F., VINCENT F. (2019), La casse du siècle : à propos des réformes de l'hôpital public, Paris, Raisons d'agir.

KALIKA M. (2018), The assessment of the managerial impact of research: the $m$ index, Document de travail, Lyon, Business Science Institute.

Kaplan S., SAdler B., LitTle K., Franz C., ORRIS P. (2012), Can sustainable hospitals help bend the health care cost curve?, Commonwealth Fund, vol. 29, p. 1-14.

KARASEK R. (1979), Job demands, job decision latitude, and mental strain: implications for job redesign, Administrative Science Quarterly, vol. 24, n² 2, p. 285-308.

KAROUI M., DUdÉzERT A. (2012), Capital social et enjeux de pouvoir : une perspective socio-politique de l'appropriation d'une technologie de réseaux sociaux au sein d'une collectivité territoriale, Systèmes d'Information \& Management, vol. $17, \mathrm{n}^{\circ} 1$, p. 49-80.

KEBRITCHI M., HIRUMI A. (2008), Examining the pedagogical foundations of modern educational computer games, Computers \& Education, vol. 51, n4, p. 1729-1743.

KELLY K. (1998), New rules for the new economy, New York, Viking Press.

KERADEC H. (2012), Épistémologie et didactique de la gestion : le cas du concept de décision, Thèse de doctorat en Sciences de Gestion, CNAM Paris. 
KIM S. (2006), Public service motivation and organizational citizenship behavior in Korea, International Journal of Manpower, vol. 27, n 8, p. 722-740.

KIPNIS K. (2001), Vulnerability in research subjects: a bioethical taxonomy, in SHAPIRO H. (dir.), Ethical and policy issues in research involving human research participants, Bethesda (MD), National Bioethics Advisory Commission, vol. 2, p. G1-G13.

KIRKPATRICK I., ACKROYD S., WALKER R. (2005), The new managerialism and public service professions: change in health, social services and housing, New York, Palgrave Macmillan.

KIRSCH L. (1996), The management of complex tasks in organization: controlling the systems development process, Organization Science, vol. 7, n 1, p. 1-21.

KNIGHTS D., StURdY A., Morgan G. (1994), The consumer rules? An examination of the rhetoric and "reality" of marketing in financial services, European Journal of Marketing, vol. 28, n³, p. 42-54.

KOENIG G. (1996), Management stratégique : paradoxes, interactions et apprentissages, Paris, Nathan.

KORPIAHO K., PÄIVIÖ H., RÄSÄNEN K. (2007), Anglo-American forms of management education: a practice-theoretical perspective, Scandinavian Journal of Management, vol. 23, n¹, p. 36-65.

KoUMENTA M. (2015), Public service motivation and organizational citizenship, Public Money \& Management, vol. 35, n5, p. 341-348.

KovatS-Bernat J.-C. (2002), Negotiating dangerous fields: pragmatic strategies for fieldwork amid violence and terror, American Anthropologist, vol. 104, $\mathrm{n}^{\circ} 1$, p. 208-222.

KRISTOF A. (1996), Person-organization fit: an integrative review of its conceptualizations, measurement, and implications, Personnel Psychology, vol. 49, n 1, p. 1-49.

KUSI-SARPONG S., GUPTA H., SARKIS J. (2019), A supply chain sustainability innovation framework and evaluation methodology, International Journal of Production Research, vol. 57, $n^{\circ} 7$, p. 1990-2008.

KWON I., SUH T. (2004), Factors affecting the level of trust and commitment in supply chain relationships, Journal of Supply Chain Management, vol. 40, n 1, p. 4-14.

LA BROISE P. (2013), Une professionnalisation dans son inverse : la déprofessionnalisation des universitaires français, Recherche \& Formation, $n^{\circ} 72$, p. 57-70.

LAROCHE H. (2007), Les relations incertaines entre pratiques et enseignement dans le domaine de la gestion, Revue française de gestion, $\mathrm{n}^{\circ} 178-179$, p. 55-70.

LAROUSSERIE D. (2020), Les avancées en trompe-l'œil de la loi de programmation de la recherche, censée empêcher le décrochage de la France, Le Monde, 16 novembre.

LAUFER R. (2020), Tocqueville aujourd'hui : crise dans la démocratie, Caen, Éditions Management \& Société.

LAUfER R., MUlLeR-LAgARde Y. (2017), Le management face au judiciaire : un nouveau domaine d'enseignement et de recherche, Revue française de gestion, $\mathrm{n}^{\circ} 269$, p. 11-17.

Lavastre O., CARBone V., Ageron B., dir. (2016), Les grands auteurs en logistique et supply chain management, Caen, Éditions Management \& Société.

LAZARUS R., FolKMAN S. (1984), Stress, appraisal and coping, New York, Springer. 
LEBRATY J. (2000), S'intéresser à la logistique : un pari scientifique, managérial et pédagogique, in FABBe-Costes N., Colin J., PAChÉ G. (dir.), Faire de la recherche en logistique et distribution ?, Paris, Vuibert-Fnege, p. 5-28.

Le Breton-Miller I., Miller D. (2021), Alienation among management scholars, Revue française de gestion, $\mathrm{n}^{\circ} 294$, p. 53-75.

LEE R. (1993), Dangerous fieldwork, Thousand Oaks (CA), Sage.

LEE Y.-O., LEE R. (2012), Methodological research on "sensitive" topics: a decade review, Bulletin of Sociological Methodology, vol. 114, n 1, p. 35-49.

Lee H., Padmanabhan V., Whang S. (1997), The bullwhip effect in supply chains, Sloan Management Review, vol. 38, n³, p. 93-102.

LE GALÈs P., SCOTT A. (2008), Une révolution britannique? Autonomie sans contrôle ou "freer markets, more rules ", Revue française de sociologie, vol. 49, $n^{\circ} 2$, p. 301-330.

Lejeune C., Starkey K., Kalika M., Tempest S. (2019), The impact of business schools: increasing the range of strategic choices, Management International, vol. 23, n², p. 88-98.

LÉPINARD P. (2019), Le projet EdUTeam : des wargames comme supports d'apprentissage expérientiel au management, Actes des $3^{\text {es }}$ Journées de Pratiques et de Recherches MACCA Management, Toulouse, p. 1-20 (CD-rom).

LÉPINARD P., VANDANGEON-DERUMEZ I. (2019), Apprendre le management autrement : la ludopédagogie au service du développement des soft skills des étudiant.e.s, Actes de la XXVIII Conférence de l'Association internationale de management stratégique, Dakar, p. 1-24.

LeTICHE H., LightFoOT G., Lilley S. (2017), Classements, capitalisme académique et affects des chercheurs en gestion, Revue française de gestion, $\mathrm{n}^{\circ} 267$, p. 97-115.

LEWIS G., Frank S. (2002), Who wants to work for the government?, Public Administration Review, vol. 62, n4, p. 395-404.

LIU B., YANG K., YU W. (2015), Work-related stressors and health-related outcomes in public service: examining the role of public service motivation, American Review of Public Administration, vol. 45, $\mathrm{n}^{\circ} 6$, p. 653-673.

LORINO P., MOTTIS N. (2020), Et l'organisation dans tout ça ? Un oubli persistant, y compris en cas de crise..., Revue française de gestion, $n^{\circ} 288$, p. 11-34.

LUSSIER S., CHANLAT J.-F. (2017), Les enseignants en gestion face aux nouvelles injonctions institutionnelles : une étude France-Québec, Revue française de gestion, $\mathrm{n}^{\circ} 267$, p. 79-95.

MacKenzie D., Millo Y. (2003), Constructing a market, performing theory: the historical sociology of a financial derivatives exchange, American Journal of Sociology, vol. 109, n²1, p. 107-145.

Mackenzie D., MuniesA F., SUI L. (2007), Do economists make markets? On the performativity of economics, Princeton (NJ), Princeton University Press.

MAESSCHALCK J., VAN DER WAL Z., HUBERTS L. (2008). Public service motivation and ethical conduct, in PerRy J., Hondeghem A. (dir.), Motivation in public management: the call of public service, Oxford, Oxford University Press, p. 157-176. 
MALINOWSKI B. (1922/2014), Argonauts of the Western Pacific: an account of native enterprise and adventure in the archipelagoes of Melanesian New Guinea, Londres, Routledge.

MARCH J. (1991), Décisions et organisations, Paris, Éditions d'Organisation.

MARCUS G. (1997), The uses of complicity in the changing mise-en-scène of anthropological fieldwork, Representations, vol. 59, p. 85-108.

MÅRTENSSON M. (2009), Recounting counting and accounting: from political arithmetic to measuring intangibles and back, Critical Perspectives on Accounting, vol. 20, $n^{\circ} 7$, p. 835-846.

MARTIN O. (2020), L'empire des chiffres : sociologie de la quantification, Paris, Armand Colin.

MARTINET A.-C. (1993), Diagnostic global et management stratégique, in MARION A. (dir.), Le diagnostic d'entreprise : cadre méthodologique, Paris, Economica, p. 199-210.

MASCLEF O. (2013), Le rôle du don et du gratuit dans l'entreprise : théories et évidences, Économies \& Sociétés, série Économie de l’Entreprise, n² 22, p. 7-31.

MAslach C., SChaufeli W., Leiter M. (2001), Job burnout, Annual Review of Psychology, vol. 52, $\mathrm{n}^{\circ} 1$, p. 397-422.

MAuss M. (1923/2012), Essai sur le don : forme et raison de l'échange dans les sociétés archaïques, Paris, Presses Universitaires de France, $2^{\mathrm{e}}$ éd.

McAfee R., Glassman M., Honeycutt JR. E. (2002), The effects of culture and human resource management policies on supply chain management strategy, Journal of Business Logistics, vol. 23, n 1, p. 1-18.

Meagher L., Lyall C., Nutley S. (2008), Flows of knowledge, expertise and influence: a method for assessing policy and practice impacts from social science research, Research Evaluation, vol. 17, n³, p. 163-173.

MEIER O. (1998), Facteurs de réussite de la réalisation d'objectifs de symbiose en phase de post-acquisition : propositions et élaboration théorique, Thèse de doctorat en Sciences de Gestion, Université Paris-12 Val-de-Marne.

MÉRINDOL J.-Y. (2008), Comment l'évaluation est arrivée dans les universités françaises, Revue d'Histoire Moderne \& Contemporaine, n55, p. 7-27.

MERINDOL J.-Y. (2010), Les universitaires et leurs statuts depuis 1968, Le Mouvement Social, $\mathrm{n}^{\circ} 233$, p. 69-91.

MERLE K. (2010), Le contrat psychologique comme clé de compréhension du suicide au travail, Humanisme \& Entreprise, $n^{\circ} 296$, p. 41-58.

Miao Q., NeWman A., YU J., XU L. (2013), The relationship between ethical leadership and unethical pro-organizational behavior: linear or curvilinear effects?, Journal of Business Ethics, vol. 116, n³, p. 641-653.

Michel H., Mc Namara P. (2014), Serious games : faites vos jeux!, Systèmes d'Information \& Management, vol. 19, n³, p. 3-8.

MikKELSEN A., OGAARD T., LOVRICH N. (2000), Modeling the effects of organizational setting and individual coping style on employees subjective health, job satisfaction and commitment, Public Administration Quarterly, vol. 24, n³, p. 371-397.

MILCENT C. (2016), Upcoding and heterogeneity in hospitals' response: a natural experiment, Working Paper $n^{\circ} 2016-13$, Paris School of Economics. 
MiLLER P. (2001), Governing by numbers: why calculative practices matter, Social Research, vol. 68, $\mathrm{n}^{\circ} 2$, p. 379-396.

MilleR P., O'LeARY T. (1987), Accounting and the construction of the governable person, Accounting, Organizations \& Society, vol. 12, n³, p. 235-265.

MoniN P. (2017), La grande transformation du métier de chercheur, Revue Internationale PME, vol. 30, n³-4, p. 7-15.

MonOd O. (2020), La déconsidération des universités par le milieu politique est structurelle : interview de Mathias Bernard, Libération, 29 décembre.

MoRGAN G. (1986/2006), Images of organization, Thousand Oaks (CA), Sage.

MORLEY L. (2005), The micropolitics of quality, Critical Quarterly, vol. 47, n²1-2, p. 83-95.

MORRISON E. (2006), Doing the job well: an investigation of pro-social rule breaking, Journal of Management, vol. 32, n 1, p. 5-28.

MousA S., ОтнmAN M. (2020), The impact of green human resource management practices on sustainable performance in healthcare organisations: a conceptual framework, Journal of Cleaner Production, vol. 243, Article 118595.

MOYNIHAN D., PANDEY S. (2007), The role of organizations in fostering public service motivation, Public Administration Review, vol. 67, n 1, p. 40-53.

Muniesa F., CALlon M. (2013), La performativité des sciences économiques, in Steiner P., Vatin F. (dir.), Traité de sociologie économique, Paris, Presses Universitaires de France, p. 281-316.

NADER L. (1972). Up the anthropologist: perspectives gained from studying up, in HyMES D. (dir.), Reinventing anthropology, New York, Pantheon, p. 284-311.

NANDE F., Commeiras N. (2020), Stratégies identitaires en réponse aux signaux de l'environnement : proposition d'une typologie et effets sur le bien-être au travail. Une étude exploratoire auprès des enseignants-chercheurs de l'université française, $@ G R H, n^{\circ} 35$, p. 73-102.

NogUeRA F. (2018), Tools and devices for intervention research: the case of intervention in human resource management, in Buono A., SAVAll H., CAPElletti L. (dir.), Intervention-research: from conceptualization to publication, Charlotte (NC), Information Age Publishing, p. 71-91.

NogueRA F., PLANE J.-M. (2020), La recherche-intervention : éléments d'épistémologie, de méthode et principes d'action, in FrImousSE S., PERETTI J.-M. (dir.), Produire du savoir et de l'action : le vade-mecum du dirigeant-chercheur, Caen, Éditions Management \& Société, p. 53-62.

OBLINGER D., dir. (2006), Learning spaces, Louisville (CO), Educause.

OCDE (2005), Manuel d'Oslo, Paris, $3^{\mathrm{e}}$ éd.

OChSNER M., Hug S., DANIEl H.-D., dir. (2016), Research and assessment in the humanities: toward criteria and procedures, Cham, Springer.

OgBonNA E., HARRIS L. (2004), Work intensification and emotional labour among UK university lecturers: an exploratory study, Organization Studies, vol. 25, $\mathrm{n}^{\circ} 7$, p. 1185-1203.

O'Hare P., Coaffee J., HAWKesworth M. (2010), Managing sensitive relations in co-produced planning research, Public Money \& Management, vol. 30, $\mathrm{n}^{\circ} 4$, p. 243-250. 
ORIOL N. (2020), Agilité et inclusion pédagogique à l'université : un retour d'expérience, Management \& Data Science, vol. 5, nº 1, Article 14395.

Osterloh M., Frey B., Homberg F. (2008), Le chercheur et l'obligation de rendre des comptes, Gérer et Comprendre, n॰91, p. 48-54.

OURIEMMI O., GÉRARD B. (2017), La responsabilisation de la Société Générale : normes juridiques et management dans le volet civil du procès Kerviel, Revue française de gestion, $\mathrm{n}^{\circ} 269$, p. 65-81.

PACHÉ G. (2002), L'évolution des relations logistiques entre industriels et détaillants : coopération ou simple coordination ?, Gestion 2000, vol. 18, n 1, p. 109-124.

PACHÉ G. (2021a), La société malade de la Covid-19 : regards logistiques croisés, Aix-en-Provence, Presses Universitaires d'Aix-Marseille.

PACHÉ G. (2021b), Menaces sur le corps des professeurs des universités en sciences de gestion et du management ?, Revue française de gestion, $n^{\circ} 294$, p. 41-51.

Pando-Garcia J., Periañez-Cañadillas I., Charterina J. (2016), Business simulation games with and without supervision: an analysis based on the TAM model, Journal of Business Research, vol. 69, $\mathrm{n}^{\circ}$ 5, p. 1731-1736.

Papa A., Mital M., Pisano P., Del Giudice M. (2020), E-health and wellbeing monitoring using smart healthcare devices: an empirical investigation, Technological Forecasting \& Social Change, vol. 153, Article 119226.

PARAdeISE C., Thoenig J.-C. (2013), Academic institutions in search of quality: local orders and global standards, Organization Studies, vol. 34, n² 2, p. 189-218.

PASIN F., Giroux H. (2011), The impact of a simulation game on operations management education, Computers \& Education, vol. 57, n 1 , p. 1240-1254.

PERCERou R., dir. (1984), Entreprise, gestion et compétitivité, Economica, Paris.

PERETTI-NDIAYE M. (2016), Race, racisme, racialisation : que nous disent les discours ?, Revue européenne des sciences sociales, n54, p. 103-128.

PERry J., WISE L. (1990), The motivational bases of public service, Public Administration Review, vol. 50, n³, p. 367-373.

PETERNELJ-TAYLOR C. (2005), Conceptualizing nursing research with offenders: another look at vulnerability, International Journal of Law \& Psychiatry, vol. $28, \mathrm{n}^{\circ} 4$, p. 348-359.

Pettigrew A. (1987), Context and action in the transformation of the firm, Journal of Management Studies, vol. 24, $n^{\circ}$ 6, p. 649-670.

PIHEL L. (2011), Et si le travail m'était conté en termes de don ?, Réalités Industrielles, $\mathrm{n}^{\circ} 1$, p. 40-45.

PLANE J.-M. (2018), From intervention-research to the production of knowledge: a summary of experimentation, in BUONO A., SAVALL H., CAPELlETTI L. (dir.), Intervention-research: from conceptualization to publication, Charlotte (NC), Information Age Publishing, p. 47-70.

Point S., El Akremi A., Igalens I. (2017), L'entrée dans la carrière d'enseignant-chercheur en GRH : quelles sont les spécificités d'accès à un poste en business school ou à l'université ?, Revue française de gestion, n²63, p. 97-119.

PoluitT C. (2009), Bureaucracies remember, post-bureaucratic organizations forget?, Public Administration, vol. 87, n² 2, p. 198-218. 
PONTILLE D., TORNY D. (2010), The controversial policies of journal ratings: evaluating social sciences and humanities, Research Evaluation, vol. 19, n5, p. 347-360.

PORTER T. (1995), Trust in numbers: the pursuit of objectivity in science and public life, Princeton (NJ), Princeton University Press.

POWER M. (1999), The audit society: rituals of verification, Oxford, Oxford University Press.

PRAS B., dir. (2010), L'évaluation des enseignants-chercheurs en sciences de gestion, Paris, FNEGE.

Prost A. (1989), 1968 : mort et naissance de l'université française, Vingtième Siècle : Revue d'Histoire, $\mathrm{n}^{\circ}$ 23, p. 59-70.

QURATULAIN S., KHAN A.-K. (2015), Red tape, resigned satisfaction, public service motivation, and negative employee attitudes and behaviors: testing a model of moderated mediation, Review of Public Personnel Administration, vol. 35, $\mathrm{n}^{\circ} 4$, p. 307-332.

RAFFouRNIER B. (2011), Discussion de " La crise de la normalisation comptable internationale, une crise intellectuelle ", Comptabilité Contrôle Audit, vol. 17, n 1 , p. 165-170.

ReAson P., BRAdBURY H., dir. (2008), Handbook of action research: participative inquiry and practice, Thousand Oaks (CA), Sage, $2^{\mathrm{e}}$ éd.

Renzetti C., Lee R., dir. (1993), Researching sensitive topics, Thousand Oaks (CA), Sage.

Reveluino S., Mouritsen J. (2015), Accounting as an engine: the performativity of calculative practices and the dynamics of innovation, Management Accounting Research, vol. 28, p. 31-49.

RichaUd L. (2016), Au parc par temps de fête : fêtes étatiques et performances publiques à Pékin aujourd'hui, L’Espace Politique, n³0, p. 1-23.

RICKLES H. (1999), Big deals are a big deal: supplier mergers may limit product choice and harm the industry companies claim to help, Modern Healthcare, vol. 29, $\mathrm{n}^{\circ} 16$, p. 52.

RicoeUR P. (2004), Parcours de la reconnaissance, Paris, Éditions Stock.

RIPOLL G., SCHOTT C. (2022), Does public service motivation foster justification of unethical behavior? Evidence from survey research among citizens, International Public Management Journal, à paraître.

Ritz A., Brewer G., Neumann O. (2016), Public service motivation: a systematic literature review and outlook, Public Administration Review, vol. 76, n³, p. 414-426.

ROSE N. (1991), Governing by numbers: figuring out democracy, Accounting, Organizations \& Society, vol. 16, n 7, p. 673-692.

ROSE D., MEYER A. (2002), Teaching every student in the digital age: universal design for learning, Alexandria (VA), Association for Supervision \& Curriculum Development.

ROUSSEAU D., MCCARTHY S. (2007), Educating managers from an evidence-based perspective, Academy of Management Learning \& Education, vol. 6, n 1, p. 84-101.

ROYER I. (2011), La responsabilité des chercheurs en gestion, Revue française de gestion, $\mathrm{n}^{\circ} 216$, p. 65-73. 
Ruscio J., Seaman F., D’Oriano C., Stremlo E., Mahalchik K. (2012), Measuring scholarly impact using modern citation-based indices, Measurement: Interdisciplinary Research \& Perspectives, vol. 10, n³, p. 123-146.

SACHET-Milliat A. (2009), La délinquance d'affaires : les défis méthodologiques des recherches en terrain sensible, Revue Internationale de Psychosociologie, $\mathrm{n}^{\circ} 35$, p. 95-115.

SACRISTE V. (2014), Le métier d'enseignant-chercheur au prisme de ses contradictions, Sociologies Pratiques, vol. 3, HS n 1, p. 53-63.

Saint-Onge S., Alis D., Wolf J., Rosenberg T. (2016), Comment améliorer la pertinence de la recherche en gestion ?, Recherches en Sciences de Gestion, ${ }^{\circ} 113$, p. 169-195.

Salas E., Wildman J., Piccolo R. (2009), Using simulation-based training to enhance management education, Academy of Management Learning \& Education, vol. 8, n 4, p. 559-573.

SALMON G. (2002), E-tivities: a key to active online learning, Londres, Routledge.

SAMPIERI-TEISSIER N., LIVOLSI L. (2019), L'hôpital dans la supply chain santé : quelle évolution des compétences managériales?, Logistique \& Management, vol. 27, $\mathrm{n}^{\circ} 1$, p. 33-43.

SANTINI A., WOERTH E. (2008), Livre blanc sur l'avenir de la fonction publique, Rapport pour le compte du Ministère du Budget, des Comptes Publics et de la Fonction Publique, Paris.

SAVALL H., ZARDET V. (1987/2020), Maîtriser les coûts et performances cachés : le contrat d'activité périodiquement négociable, Paris, Economica, $7^{\mathrm{e}}$ éd.

SAVAll H., Goter F. (2021), Comment évaluer les impacts socio-économiques d'un dispositif public ?, Observatoire de l'ASAP, 10 février.

SAVAll H., ZARDet V., Bonnet M., CAPPelletti L. (2019), Valoriser la recherche par l'expérimentation en entreprise : cas du modèle de management socio-économique, Revue française de gestion, $\mathrm{n}^{\circ} 284$, p. 149-169.

SAWYER B. (2002), Serious games: improving public policy through game-based learning and simulation, Washington DC, Woodrow Wilson International Center for Scholars.

SChNapP A., VIDAL-NAQUet P. (1969/2018), Journal de la Commune étudiante : textes et documents, novembre 1967-juin 1968, Paris, Le Seuil, $3^{e}$ éd.

SCHNEIDER B., Goldstiein H., SMITH D. (1995). The ASA framework: an update, Personnel Psychology, vol. 48, n4, p. 747-773.

SCHOEMAKER P. (2008), The future challenges of business: rethinking management education and research, California Management Review, vol. 50, n³, p. 119-139.

SCHOTT C., RITZ A. (2018), The dark sides of public service motivation: a multi-level theoretical framework, Perspectives on Public Management \& Governance, vol. 1, $\mathrm{n}^{\circ} 1$, p. 29-42.

SECUNDO G., SHAMS R., NucCI F. (2021), Digital technologies and collective intelligence for healthcare ecosystem: optimizing Internet of Things adoption for pandemic management, Journal of Business Research, vol. 131, p. 563-572. 
SHUTE V., KIM Y.-J. (2014), Formative and stealth assessment, in SPECTOR J., MerRILl M., Elen J., BISHOP M. (dir.), Handbook of research on educational communications and technology, New York, Springer, p. 311-321

SieBer J., StANLEY B. (1988), Ethical and professional dimensions of socially sensitive research, American Psychologist, vol. 43, n 1, p. 49-55.

SIEGRIST J. (1996), Adverse health effects of high-effort/low-reward conditions, Journal of Occupational Health Psychology, vol. 1, n²1, p. 27-41.

SIEGRIST J. (2012), Risques psychosociaux au travail et santé : progrès scientifiques et futurs défis, in COURTET C., Gollac M. (dir.), Risques du travail, la santé négociée, Paris, La Découverte, p. 161-172.

SiEgRIST J., WAHRENDORF M., dir. (2016), Work stress and health in a globalized economy: the model of effort reward imbalance, Cham, Springer.

SIMCHI-LeVI D., CHEN X., BRAMEL J. (2014), The logic of logistics: theory, algorithms, and applications for logistics management, New York, Springer, $3^{\mathrm{e}}$ éd.

SIMON H. (1958), The role of expectations in an adaptative or behavioristic model, in BOWMAN M. (dir.), Expectations, uncertainty and business behavior, New York, Social Science Research Council, p. 49-58.

SIMON H. (1976), From substantive to procedural rationality, in LATSIS S. (dir.), Method and appraisal in economics, Cambridge (MA), Cambridge University Press, p. 129-148.

SIMON H. (1997), Models of bounded rationality, Cambridge (MA), MIT Press.

Simons R. (1995), Control in an age of empowerment, Harvard Business Review, vol. 73, n², p. 80-88.

SINGH S.-K., EL-KASSAR A.-N. (2019), Role of big data analytics in developing sustainable capabilities, Journal of Cleaner Production, vol. 213, p. 1264-1273.

SLUKA J. (1990), Participant observation in violent social contexts, Human Organization, vol. 49, n², p. 114-126.

Somers R., Mannheimer D., Kleman M., Mellinger G. (1982), Structured interviews: technical and ethical problems, in SMITH R., MANNING P. (dir.), Handbook of social science methods. Volume 2: Qualitative methods, Cambridge (MA), Ballinger, p. 145-161.

SONNETTE M. (2020), Loi sur la recherche : universités et laboratoires dans la rue contre la précarité, Le Monde, 4 mars.

SOROKIN P. (1956). Fads and foibles in modern sociology and related sciences, Chicago (IL), Henry Regnery Co.

STARBuck W. (2005). How much better are the most-prestigious journals? The statistics of academic publication, Organization Science, vol. 16, n² 2, p. 180-200.

STARKEY K., MADAN P. (2001), Bridging the relevance gap: aligning stakeholders in the future of management research, British Journal of Management, vol. 12, $\mathrm{n}^{\circ} 1$, p. S3-S26.

STAZYK E., DAVIS R. (2015), Taking the "high road": does public service motivation alter ethical decision making processes?, Public Administration, vol. 93, $\mathrm{n}^{\circ} 3$, p. 627-645.

STOCK J., BOYER S., HARMON T. (2010), Research opportunities in supply chain management, Journal of the Academy of Marketing Science, vol. 38, n 1, p. 32-41. 
Sutton R., StAW B. (1995), What theory is not, Administrative Science Quarterly, vol. 40, n³, p. 371-384.

TANIGUCHI E. (2014), Concepts of city logistics for sustainable and liveable cities, Procedia-Social \& Behavioral Sciences, vol. 151, p. 310-317.

THOMAS J. (1993), Doing critical ethnography, Thousand Oaks (CA), Sage.

TITMUSS R. (1970/2018), The gift relationship: from human blood to social policy, Bristol, Policy Press.

TiXier D., MATHE H., Colin J. (1996), La logistique d'entreprise : vers un management plus compétitif, Paris, Dunod, $2^{\mathrm{e}}$ éd.

UMPHRESS E., BINGHAM J. (2011), When employees do bad things for good reasons: examining unethical pro-organizational behaviors, Organization Science, vol. 22, n³, p. 621-640.

VANDENABEELE W. (2007), Toward a public administration theory of public service motivation: an institutional approach, Public Management Review, vol. 9, $\mathrm{n}^{\circ} 4$, p. 545-556.

VANDENABEelE W. (2009), The mediating effect of job satisfaction and organizational commitment on self-reported performance: more robust evidence of the PSM-performance relationship, International Review of Administrative Sciences, vol. 75, n 1, p. 11-34.

VANDENABeELE W. (2014), Explaining public service motivation: the role of leadership and basic needs satisfaction, Review of Public Personnel Administration, vol. 34, n², p. 153-173.

Vandenabeele W., Ritz A., Neumann O. (2018), Public service motivation: state of the art and conceptual cleanup, in ONGARO E., VAN ThIEL S. (dir.), The Palgrave handbook of public administration and management in Europe, Cham, Springer, p. 261-278.

VAN DE Ven A., Johnson P. (2006), Knowledge for theory and practice, Academy of Management Review, vol. 31, $\mathrm{n}^{\circ} 4$, p. 802-821.

VANGRUNDERBEEK P. (2020), Concevoir un enseignement comodal, quelles différences avec un dispositif hybride?, Document de travail, Louvain-la-Neuve, Louvain Learning Lab.

VAN Loon N., VANDENABEele W., LeISINK P. (2015), On the bright and dark side of public service motivation: the relationship between PSM and employee wellbeing, Public Money \& Management, vol. 35, n5, p. 349-356.

VAtin F., Caillé A., FaVereau O. (2010), Réflexions croisées sur la mesure et l'incertitude, Revue française de gestion, $\mathrm{n}^{\circ} 203$, p. 163-181.

Verstraete T., PHILIPPART P. (2019), La valorisation sociétale et managériale de la recherche en gestion, Revue française de gestion, $n^{\circ} 284$, p. 77-102.

VItARI C., PILlet J.-C. (2019), Le réseau social de la communauté francophone des chercheurs en systèmes d'information, Systèmes d'Information \& Management, vol. 24, $\mathrm{n}^{\circ} 1$, p. 87-105.

VIVIANI J.-L., Vo T. (2016), Peut-on arrêter de jouer au " maillon faible " dans la chaîne logistique? Le financement collaboratif du BFR dans une chaîne logistique est-il possible? in BIRonneaU L., VIVIANI J.-L. (dir.), Collaborations et réseaux : 
approches transversales en management, Rennes, Presses Universitaires de Rennes, p. 151-170.

Volimer H. (2007), How to do more with numbers? Elementary stakes, framing, keying, and the three-dimensional character of numerical signs, Accounting, Organizations \& Society, vol. 32, $n^{\circ}$ 6, p. 577-600.

WALTHER B., LARSEN L. (2020), Gamification and beyond: the case of ludification, in MARfisi-Schottman I., Bellotti F., HAMON L., Klemke R. (dir.), Games and learning alliance, Cham, Springer, p. 125-134.

WANG Y., HAN J.-H., BEYNON-DAVIES P. (2019), Understanding blockchain technology for future supply chains: a systematic literature review and research agenda, Supply Chain Management, vol. 24, n 1, p. 62-84.

WAX R. (1986), Doing fieldwork: warnings and advice, Chicago (IL), Chicago University Press.

WeBer M. (1919/2002), Le savant et le politique, Paris, 10/18.

Weber M., Rodhain F., Fallery B. (2019), Usage de la réalité virtuelle et développement individuel des enseignants-chercheurs : une approche par la didactique professionnelle, Management \& Avenir, $\mathrm{n}^{\circ} 112$, p. 37-57.

WEINGART P. (2005), Impact of bibliometrics upon the science system: inadvertent consequences?, Scientometrics, vol. 62, n 1, p. 117-131.

Weissmüller K.-S., De Waele L., van Witteloostuijn A. (2022), Public service motivation and prosocial rule-breaking: an international vignettes study in Belgium, Germany, and the Netherlands, Review of Public Personnel Administration, à paraitre.

WILLIAMSON O. (1991), Comparative economic organization: the analysis of discrete structural alternatives, Administrative Science Quarterly, vol. 36, $\mathrm{n}^{\circ} 2$, p. 269-296.

Willmott H. (1995), Managing the academics: commodification and control in the development of university education in the UK, Human Relations, vol. 48, $n^{\circ} 9$, p. 993-1027.

WOUTERS P. (1999), The citation culture, Unpublished doctoral dissertation, University of Amsterdam.

XU X., CHEN X., JIA F., BRown S., Gong Y., XU Y. (2018), Supply chain finance: a systematic literature review and bibliometric analysis, International Journal of Production Economics, vol. 204, p. 160-173.

YORDANOVA Z. (2019), Educational innovation: bringing back fads to fundamentals, Proceedings of the ISPIM Innovation Conference, Florence, p. 1-9 (CD-rom).

YOUSSEF E., AUDRAN J. (2019), La personnalisation de l'apprentissage vue comme facteur effectif d'innovation pédagogique. Spirale : Revue de Recherches en Éducation, vol. 1, n 1 , p. 157-172.

ZARKA Y.-C. (2009), L'évaluation : un pouvoir supposé savoir, Cités, n³7, p. 113-123.

ZWICK T. (2002), Employee resistance against innovations, International Journal of Manpower, vol. 23, $n^{\circ} 6$, p. 542-552. 


\section{Les auteurs}

Blandine Ageron est professeur des universités en sciences de gestion et du management à I'Université Grenoble Alpes où, après avoir été responsable de la Licence professionnelle Management des approvisionnements et de la logistique au sein de I'IUT de Valence, elle occupe la fonction de chef du département Techniques de Commercialisation. Membre du Centre d'études et de recherches appliquées à la gestion (CERAG), ses travaux de recherche portent sur la conception et le management des chaînes logistiques durables, les innovations au sein des relations inter-organisationnelles, la gestion des compétences dans le SCM, et l'attractivité des métiers de la logistique et du SCM. Elle est rédactrice en chef de Logistique \& Management depuis 2019, et membre élue du bureau de l'Association internationale de recherche en logistique et supply chain management (AIRL-SCM).

Thérèse ALBERTINI est maître de conférences HDR en sciences de gestion et du management à I'Université de Corse Pascal-Paoli où elle enseigne au sein de I'IAE et de l'IUT. Depuis le début de sa carrière, elle est très investie dans son établissement (élue dans de nombreux conseils de l'université, chef de département, création et responsabilités de diplômes, encadrements, participation aux comités de sélection, etc.). Elle est actuellement directrice adjointe du laboratoire Lieux, Identités, eSpace et Activités (LISA UMR CNRS 6240). Spécialiste de marketing, ses travaux ont fait l'objet de publications dans des revues et des colloques; ils portent notamment sur l'ancrage régional comme pratiques de différenciation dans les comportements et stratégies des acteurs.

Nicolas AUBERT est professeur des universités en sciences de gestion et du management à Aix-Marseille Université, en poste à l'IAE où il occupe la fonction de Faculty Fellow. Mentor de I'Institut de Recherche sur l'Actionnariat Salarié et le Partage des Profits de l'Université Rutgers, il est chercheur associé au Groupe de recherche en anthropologie chrétienne et entreprise (GRACE) et à I'Institut français de gouvernement d'entreprise (IFGE), et participe au conseil scientifique de la Fédération française des associations d'actionnaires salariés et anciens salariés (FAS). Spécialiste de finance, notamment en gouvernance salariale, une thématique ayant donné lieu à de nombreux articles académiques, il a dirigé le Centre d'études et de recherche en gestion d'Aix-Marseille (CERGAM) et a été directeur adjoint du Groupe de recherche en management (GRM). 
Hanane BEDDI est professeur des universités en sciences de gestion et du management à l'Université Paris 8 Vincennes-Saint-Denis où elle enseigne au sein de la Faculté d'économie et de gestion. Membre du Laboratoire d'économie dionysien (LED) et membre élue du conseil d'administration de l'Association francophone de management international (ATLAS-AFMI), ses travaux portent sur les relations siège / filiales dans les firmes multinationales, en s'intéressant en particulier au rôle du siège. Elle mène également des recherches sur l'internationalisation des PME, sous l'angle notamment de l'entrepreneuriat international. Sur ces thématiques, elle est l'auteur de nombreuses publications dans des revues scientifiques et dans des colloques.

Omar BentahAR est maître de conférences HDR en sciences de gestion et du management à I'Université de Lorraine, en poste à l'IAE de Metz où il est responsable du Master Management et administration des entreprises et créateur / directeur du Master 2 Management de projet. Chercheur au Centre européen de recherche en économie financière et en gestion des entreprises (CEREFIGE), il est co-fondateur de la conférence internationale PROLOG (Project \& Logistics). Ses recherches, portant sur le management des projets complexes, l'innovation supply chain et la logistique de la santé, ont été publiées notamment dans IEEE Transactions on Engineering Management, Technological Forecasting \& Social Change et Logistique \& Management.

Smail BENzIDIA est maître de conférences HDR en sciences de gestion et du management à I'Université de Lorraine, en poste à l'IAE de Metz où il est responsable du Master Management, Master Management de la qualité et responsable académique du développement des relations internationales. Chercheur au Centre européen de recherche en économie financière et en gestion des entreprises (CEREFIGE), co-fondateur de la conférence internationale PROLOG (Project \& Logistics), ses travaux portant sur les systèmes d'information inter-organisationnels et le supply chain management ont donné lieu à la publication de nombreux articles et à la présentation de communications lors de conférences nationales et internationales.

Delphine BERENI est maître de conférences en sciences de gestion et du management à I'Université de Corse Pascal-Paoli, où elle est directrice de l'IAE de Corte, en y ayant créé des diplômes, instauré l'apprentissage et, depuis le début de sa carrière, assuré de multiples responsabilités pédagogiques. Impliquée dans la vie universitaire, elle participe à des conseils d'établissement (Commission de la recherche et Conseil académique) et de composante (Conseil d'administration). Membre du laboratoire Lieux, Identités, eSpace et Activités (LISA UMR CNRS 6240), ses travaux et publications scientifiques portent sur le comportement du consommateur (achats responsables, alimentaires et infantiles, attachement aux produits régionaux) et les stratégies des acteurs (ancrage régional, stratégie de différentiation, marques collectives).

Marc BIDAN est professeur des universités en sciences de gestion et du management à l'Université de Nantes, en poste au département Homme Entreprise Société de Polytech Nantes. Membre du Laboratoire d'économie et de management de Nantes Atlantique (LEMNA), ancien président de l'Association information \& management 
(AIM) et membre du jury national du diplôme d'expertise comptable en charge de l'épreuve de management des systèmes d'information, ses travaux portent sur l'intégration et l'externalisation des systèmes d'information et la sobriété numérique. II mène également des recherches sur les écosystèmes d'affaires portés par le numérique en Afrique subsaharienne. II est l'auteur de publications et production scientifiques, à la fois académiques, praticiennes et grand public, sur les technologies de l'information et de leurs processus d'appropriation et de contournement.

Marc Bollecker est professeur des universités en sciences de gestion et du management à I'Université de Haute-Alsace et directeur adjoint du Centre de recherche en gestion des organisations (CREGO). II dirige actuellement le Master Contrôle de gestion et audit organisationnel. Ses travaux ont fait l'objet d'une cinquantaine de publications dans des revues scientifiques, dans des colloques et congrès. Ils sont ancrés en contrôle de gestion et en management public et portent notamment sur l'appropriation des outils de contrôle, les pratiques de transparence, le déploiement du new public management à l'université, les caractéristiques du contrôle dans les organisations pluralistes publiques.

Marc BonNET est professeur émérite des universités en sciences de gestion et du management à I'Université Jean-Moulin Lyon III, où il a été en poste à I'IAE de Lyon. Directeur adjoint de l'Institut de socio-économie des entreprises et des organisations (ISEOR), il travaille dans le domaine des recherches-interventions socio-économiques, en ayant de nombreuses activités à l'international, notamment à l'Academy of Management où il pilote des projets dans le domaine des doctorats professionnels (Executive PhD, DBA, Doctorates in Management, etc.). II anime également des groupes de réflexion sur l'évolution de la profession d'enseignantchercheur en management, dans la continuité des travaux d'Andy Van de Ven. Ses publications, portant notamment sur les résultats des recherches-interventions qualimétriques, ont donné lieu à la publication de nombreux ouvrages et articles.

Kirsten BURKHARDT est maître de conférences HDR en sciences de gestion et du management à l'Université de Bourgogne, où elle assure la responsabilité pédagogique de I'International Master in Business Studies à l'IAE. Membre du Centre de recherche en gestion des organisations (CREGO), où elle est en charge de l'axe de recherche transversal " Entrepreneuriat / PME » et co-dirige l'axe de recherche disciplinaire en finance (FARGO), elle anime également un groupe de lecture en épistémologie en sciences de gestion. Ses recherches et publications académiques portent sur la gouvernance entrepreneuriale et la croissance des PME.

Sophie Cros est professeur des universités en sciences de gestion et du management à l'Université Le Havre-Normandie, en poste à l'Institut supérieur d'études logistiques (ISEL). Directrice déléguée du laboratoire Normandie Innovation Marché Entreprise Consommation (NIMEC), ses thématiques de recherche concernent principalement la prise de décision, le diagnostic des risques et l'anticipation de l'improbable (dans les domaines de la sécurité, de la défense et de l'éducation des populations), la logistique et l'environnement. Ses travaux ont donné lieu à de nombreux articles et communications, et elle a, en outre, co-coordonné l'ouvrage collectif Les ports en 
France : quelle stratégie portuaire pour un développement de l'activité ?, publié en 2021 aux Éditions Eska.

Mickaël DAVID est maître de conférences en sciences de gestion et du management à I'Université de Tours. En poste à I'IAE, membre de Vallorem, ses recherches sont centrées sur les usages des systèmes et des technologies de l'information, et notamment sur leur capacité à transformer les organisations. Il a publié de nombreux articles dans des revues classées comme Systèmes d'Information \& Management, Finance Contrôle Stratégie, Computers in Industry, Journal of Decision Systems, et présenté des communications lors des colloques de l'Association Information \& Management, des European Conferences on Information Systems, ou encore des International Conferences on Product Lifecycle Management.

Jean-Philippe DeNIS est professeur des universités en sciences de gestion et du management à l'Université Paris-Saclay, où il dirige le Master Management stratégique, et en particulier le Master in Management (research), formation issue d'un partenariat entre la Faculté Droit-Économie-Gestion de l'Université Paris-Saclay, l'École normale supérieure Paris-Saclay, Mines ParisTech (PSL Paris Université), I'ESCP Business School et le Centre i3-CRG de l'École polytechnique (Institut polytechnique de Paris). Membre du laboratoire Réseaux, Innovation, Territoires, Mondialisation (RITM), où il poursuit des travaux en management stratégique, il a été rédacteur en chef de la Revue Française de Gestion de 2013 à 2021. II a également initié un partenariat de valorisation scientifique en collaboration avec Xerfi Canal Productions, "IQSOG - L'innovation par la valorisation » [https://www. iqsog.org/], qui a donné lieu à la diffusion de plus de 800 interviews de recherche.

Aude Deville est professeur des universités en sciences de gestion et du management à l'Université Côte d'Azur, en poste à I'IAE de Nice où elle dirige le Master Contrôle de gestion et audit organisationnel, après avoir créé et dirigé le Groupe de recherche en management (GRM), en assurant au niveau national la fonction de délégué scientifique AERES, puis HCERES. Ancienne co-rédactrice en chef de Comptabilité Contrôle Audit et de deux dossiers spéciaux de la Revue Française de Gestion, elle a publié de nombreux articles dans des revues nationales et internationales, notamment Management Accounting Research, Comptabilité Contrôle Audit, Revue Française de Gestion, Finance Contrôle Stratégie, Journal of Applied Accounting Research et Applied Economics, et a organisé la conférence de I'Association francophone de comptabilité en 2010. Elle est présidente de la section 06 du CNU pour la mandature 2019-2023.

Jérôme DupuIs, après avoir été cadre dirigeant de collectivités locales et conseil en gestion des organisations publiques, est maître de conférences honoraire en sciences de gestion et du management à l'Université de Lille, où il a dirigé le Master Manager territorial. Ses travaux de recherche portent sur l'analyse comparée des systèmes locaux de performance au niveau européen et sur l'innovation des politiques et organisations publiques territoriales. II est l'auteur d'une quinzaine d'articles dans des revues classées, de plus de 30 ouvrages, et de plus d'une centaine de contributions à des revues professionnelles. Il est membre du bureau 
de l'Association internationale de recherche en management public (AIRMAP) en charge des partenariats et des relations extérieures en France et en Europe.

François Fulconis est maître de conférences en sciences de gestion et du management à Avignon Université. Membre associé au laboratoire Biens, Normes, Contrats (LBNC) et membre permanent du Centre de recherche sur le transport et la logistique (CRET-LOG) à Aix-Marseille Université, il est aussi membre élu du bureau de l'Association internationale de recherche en logistique et supply chain management (AIRL-SCM), membre de I'International network on new party logistics (IN-NPL) et ancien du CEFAG (FNEGE). Ses recherches portent principalement sur le management stratégique des réseaux d'entreprises et des chaînes logistiques multi-acteurs, l'intermédiation logistique et l'industrie de la prestation de services logistiques. Sur ces thématiques, il est l'auteur de plus de 200 publications académiques et de vulgarisation, nationales et internationales.

Élodie GARDET est maître de conférences en sciences de gestion et du management à I'Université Savoie Mont Blanc, en poste en école d'ingénieurs Polytech Annecy-Chambéry, responsable des enseignements SHES. Membre de I'Institut de recherche en économie et gestion (IREGE), au sein de l'équipe "Innovation et développement des organisations ", ses recherches en management stratégique et en management de l'innovation portent sur les modes de coordination instaurés dans le cadre de relations inter-organisationnelles et sur les différentes dimensions de l'innovation dans les services, plus spécifiquement dans le secteur bancaire et du tourisme. Sur ces thématiques, elle est l'auteur de nombreuses publications dans des revues académiques, de vulgarisation et dans des conférences nationales et internationales.

Irène GeORGescu est professeur des universités en sciences de gestion et du management à l'Université de Montpellier, en poste à l'Institut Montpellier Management où elle est responsable de la chaire Innov'EreS de la Key Initiative Muse sur les risques climatiques et organisations de santé. Membre de l'Association francophone de comptabilité (AFC), dont elle a été secrétaire générale, et de I'Association de recherche en management des organisations de sante (ARAMOS), dont elle fut présidente, ses travaux se situent à l'interface du contrôle de gestion et des ressources humaines dans le domaine des organisations de santé. Elle est l'auteur de plusieurs publications nationales et internationales.

Françoise Goter est maître de conférences en sciences de gestion et du management à I'Université Jean-Moulin Lyon III, en poste à I'IAE de Lyon où elle est responsable du Master 1 en alternance Management des équipes et qualité. Responsable de programme au sein de l'Institut de socio-économie des entreprises et des organisations (ISEOR), chargée de l'encadrement d'équipes d'intervenantschercheurs et du pilotage de recherches-interventions conduites au sein d'entreprises et organisations publiques ou privées, membre du laboratoire Magellan, ses travaux portent sur la modernisation du management des organisations de service public, le redressement économique des entreprises, les performances socio-économiques de TPE en profession libérale ainsi que les pratiques managériales au sein des 
entreprises familiales. Sur ces thèmes, elle a publié une cinquantaine d'articles et de communications présentées dans des congrès en France et à l'étranger.

Anne Goujon Belghit est maître de conférences en sciences de gestion et du management à I'Université de Bordeaux, où elle enseigne au sein de I'IAE. Titulaire adjointe de la chaire du capital humain et de la performance globale de Bordeaux, membre de l'Association francophone de gestion des ressources humaines (AGRH) et de l'Association nationale des docteurs es sciences économiques et en sciences de gestion, co-rédactrice en chef de Vie \& Sciences de l'Entreprise, ses travaux portent sur la gestion des ressources humaines, et tout particulièrement sur la qualité de la relation à l'emploi. Elle est l'auteur d'une vingtaine d'articles publiés dans des revues internationales comme nationales, et de deux ouvrages en management et sur le capital humain.

Anouk GREVIN est maître de conférences HDR en sciences de gestion et du management à l'Université de Nantes, en poste au département Homme Entreprise Société de Polytech Nantes. Membre du Laboratoire d'économie et de management de Nantes-Atlantique (LEMNA), au sein de l'axe "Transformations du travail ", ses travaux en organisation et GRH portent principalement sur les dynamiques de don au travail et leur reconnaissance. Ils ont donné lieu à plusieurs ouvrages, communications et articles publiés dans des revues nationales et internationales.

François GrIMA est professeur des universités en sciences de gestion et du management à l'Université Paris-Est, où il enseigne au sein de AEI International School. Membre de I'Institut de recherche en gestion (IRG), du Laboratoire interdisciplinaire d'étude du politique Hannah Arendt et de l'observatoire Action sociétale et action publique (ASAP), ses travaux portent sur la gestion des ressources humaines, et tout particulièrement l'analyse des transitions professionnelles, de la gestion de la flexibilité et des innovations organisationnelles. Il est l'auteur d'une cinquantaine d'articles de recherche dans des revues internationales comme nationales. Expert HCERES, il est également membre du jury de l'agrégation du secondaire en Économie \& Gestion.

Solange HeRnANDEz est professeur des universités en sciences de gestion et du management à Aix-Marseille Université, en poste à I'Institut de management public et gouvernance territoriale (IMPGT). Membre du Centre d'études et de recherche en gestion d'Aix-Marseille (CERGAM), ses recherches portent principalement sur le management territorial stratégique, la gestion de l'environnement et les paradoxes dans les organisations, en effectuant fréquemment des comparaisons internationales (Europe, Méditerranée). Auteur d'une trentaine d'articles scientifiques et de chapitres d'ouvrages, et d'une cinquantaine de communications, elle a également publié À la recherche du management territorial : construire les territoires entre idéologie, paradoxe et management en 2017, aux Presses Universitaires de Provence, ouvrage labellisé par la FNEGE dans la catégorie « essai ».

Jocelyn HUSSER est professeur des universités en sciences de gestion et du management à Aix-Marseille Université, où il enseigne au sein de l'IAE. Directeur de l'axe Finance Contrôle Comptabilité au sein du Centre d'études et de recherche en 
gestion d'Aix-Marseille Université (CERGAM), co-rédacteur en chef de Vie \& Sciences de l'Entreprise, il est président de l'ADERSE depuis novembre 2021. Ses travaux portent sur la RSE, et tout particulièrement sur la prise de décision éthique des professionnels en situation dilemme. Il est l'auteur d'une vingtaine d'articles publiés dans des revues internationales comme nationales, et de deux ouvrages en contrôle de gestion.

Jean-Fabrice LEBRATY est professeur des universités en sciences de gestion et du management à I'Université Jean-Moulin Lyon III, où il est en poste à I'IAE de Lyon. Membre du laboratoire Magellan, il est spécialisé en management des systèmes d'information, avec des domaines de recherche concernant la prise de décision en situation extrême, le crowdsourcing et les technologies innovantes comme la blockchain. Sur ces thématiques, il est l'auteur de nombreux articles et de plusieurs ouvrages de référence. Il est membre élu du bureau de la section 06 du CNU pour la mandature 2019-2023.

Marine LegALl-Ely est professeur des universités en sciences de gestion et du management à l'Université de Bretagne Occidentale, où elle occupe la fonction de première vice-présidente, en charge du Conseil d'administration et du Conseil académique depuis avril 2016, de secrétaire de la fondation UBO et de représentante des universités bretonnes au CESER de Bretagne. Ancienne directrice de laboratoire et directrice adjointe d'École doctorale, membre permanente du Laboratoire d'économie et de gestion de l'Ouest (LEGO), elle mène des recherches sur le prix, la gratuité et le don en comportement du consommateur et en marketing. Ses travaux ont été publiés dans les revues internationales, notamment Journal of Business Research, Journal of Marketing \& Public Policy, International Journal of Nonprofit \& Voluntary Sector Marketing, International Journal of Arts Management, Management International, et des revues françaises, notamment Recherche \& Applications Marketing, Décisions Marketing, Revue Française de Gestion et Revue des Sciences de Gestion.

Jean-François LEMOINE est professeur des universités en sciences de gestion et du management à l'Université Paris 1 Panthéon-Sorbonne et à l'ESSCA School of Management. Directeur de l'École doctorale de management Panthéon-Sorbonne, et ancien rédacteur en chef associé de Décisions Marketing, il organise chaque année, depuis 2002, le colloque sur le marketing digital (www.colloquemarketingdigital. com). Ses travaux, publiés entre autres dans Administrative Science Quarterly, Décisions Marketing, International Journal of Retail \& Distribution Management, Journal of Marketing Management, Revue Française de Gestion, Management \& Avenir et Recherche \& Applications en Marketing portent sur le marketing digital, notamment la e-shopping experience, l'influence des composantes atmosphériques d'un site Web sur le comportement des internautes, la privacy et le crowdsourcing.

Nicolas LESCA est professeur des universités en sciences de gestion et du management à I'Université Grenoble Alpes, où il enseigne à I'IUT2 de Grenoble. Après deux mandats de vice-président Formation et Vie Universitaire, il est administrateur du département Techniques de Commercialisation, directeur adjoint de l'École Universitaire de Technologie et membre du Centre d'études et de recherches 
appliquées à la gestion (CERAG). Ses travaux de recherche portent sur l'anticipation des événements imprévus et inattendus, les signaux faibles et la veille stratégique. Sur ces thèmes, il est l'auteur de nombreux ouvrages, communications et articles dans des revues académiques internationales.

Olivier Meier est professeur des universités en sciences de gestion et du management à I'Université Paris-Est. Membre du Laboratoire interdisciplinaire d'étude du politique Hannah Arendt et directeur de l'observatoire Action sociétale et action publique (ASAP), ses travaux portent sur les stratégies d'entreprise, le management interculturel et les apports de la sociologie à l'analyse des processus d'innovation et de changement. II est l'auteur d'une soixantaine d'articles dans des revues nationales et internationales, et d'une vingtaine d'ouvrages. II a reçu plusieurs prix et distinctions scientifiques, dont le prix de la meilleure communication décerné par la Fondation Paris Dauphine (Cercle de l'Innovation), et celui du meilleur article international publié dans la revue Family Business Review.

Jérémy MORVAN est maître de conférences en sciences de gestion et du management à I'Université de Bretagne Occidentale. Ancien directeur de I'IAE de Brest, il est vice-président de son université depuis cinq ans, en charge de la valorisation. Ses thématiques de recherche portent, en finance de marché, sur les covered bonds et les mécanismes de répétition des crises financières et, en finance d'entreprise, sur la relation entre finance et marketing. Ses travaux ont été publiés notamment dans Bankers, Market \& Investors, Recherche \& Applications en Marketing, et dans des ouvrages publiés aux éditions Dunod.

Emmanuelle NÈGRE est maître de conférences en sciences de gestion et du management à I'Université Toulouse 1 Capitole, en poste à la Toulouse School of Management. Membre du laboratoire TSM Research, ses recherches s'inscrivent principalement dans le domaine de la comptabilité sociétale et de la comptabilité critique. Elles ont été publiées dans des revues nationales et internationales comme Comptabilité Contrôle Audit, Critical Perspectives on Accounting, Journal of Accounting \& Public Policy et M@n@gement. Elle est membre élue du bureau de la section 06 du CNU pour la mandature 2019-2023.

Florence Noguera est professeur des universités en sciences de gestion et du management à l'Université Paul-Valéry Montpellier 3, où elle dirige le Master 2 Audit social RH, l'Executive MBA leadership, gouvernance et performance des équipes et I'Executive DBA. Membre du laboratoire Communication, Ressources Humaines \& Intervention sociale (CORHIS), elle est également secrétaire générale de l'AGRH et participe à plusieurs comités scientifiques de revues de référence dans le champ de la GRH. Ses travaux, portant sur la mesure de la performance et la normalisation de la GRH dans des activités de services et des organisations publiques, ont donné lieu à plus de 150 publications sous forme d'articles, ouvrages, communications et rapports de recherche. Elle a été primée " best paper award " par l'Academy of Management à Chicago, en 2009, pour une recherche portant sur le thème du capital humain. 
Nathalie ORIOL est maître de conférences en sciences de gestion et du management à l'Université Côte d'Azur. Membre du Groupement de recherche en droit, économie et gestion (GREDEG) et de l'École universitaire de recherche Economics, Law \& Management of Innovation, ses recherches s'intéressent à la réglementation et I'histoire de la finance, ainsi qu'aux usages des systèmes d'information au sein des pratiques financières. Ses travaux ont notamment été publiés dans Quantitative Finance, Système d'Information \& Management, Information Economics \& Policy, Economic Modelling et la Revue Française de Gestion. Elle est également membre du groupe de travail thématique Learning \& Teaching 2021 de l’European University Association.

Gilles PACHÉ est professeur des universités en sciences de gestion et du management à Aix-Marseille Université. Ancien directeur d'Aix-Marseille Université Éditions et président de l'Association internationale de recherche en logistique et supply chain management (AIRL-SCM), membre du Centre d'études et de recherche en gestion d'Aix-Marseille (CERGAM) et du bureau de l'Association Étienne Thil, il a publié ou coordonné une vingtaine d'ouvrages académiques, dont La société malade de la Covid-19 : regards logistiques croisés (Presses Universitaires d'Aix-Marseille, 2021). II est également l'auteur de plusieurs centaines d'articles, communications, chapitres d'ouvrage et rapports à destination d'organismes publics. Ses thématiques de recherche concernent principalement le management des services, la gouvernance des clubs de football et le fonctionnement des chaînes logistiques.

Évelyne Poincelot est professeur des universités en sciences de gestion et du management à l'Université de Franche-Comté où elle y dirige l'antenne du Centre de recherche en gestion des organisations (CREGO) et gère le Master Management administratif et financier en entreprise, à la fois en enseignement à distance et en apprentissage. Ses recherches portent sur la finance d'entreprises (étude de la structure financière, des rachats d'actions, des introductions en bourse, des dividendes, etc.), et les travaux en résultant ont notamment été publiés dans la Bankers, Market \& Investors, la Revue Française de Gestion, Finance Contrôle Stratégie, et Comptabilité Contrôle Audit.

Caroline RıcHÉ est maître de conférences en sciences de gestion et du management à I'Université Paris-Saclay, en poste à I'IUT de Sceaux où elle responsable de la Licence 3 Économie-Gestion, parcours Comptabilité contrôle. Membre du laboratoire Réseaux, Innovation, Territoires, Mondialisation (RITM), ses travaux de recherche s'inscrivent en comportement du consommateur et visent notamment à mieux comprendre les interactions sociales sous-jacentes à une décision collective et à une consommation partagée. Sur ces thématiques, elle est l'auteur de nombreuses publications dans des revues académiques et de vulgarisation et dans des conférences nationales et internationales. Elle est également rédactrice en chef de Management \& Data Science, revue scientifique de l'Association Information \& Management qui analyse l'impact de la transformation digitale sur le management (stratégie, marketing, ressources humaines, etc.). Elle est membre élue du bureau de la section 06 du CNU pour la mandature 2019-2023. 
Olivier RoQUES est maître de conférences HDR en sciences de gestion et du management à Aix-Marseille Université. Directeur de l'axe Stratégie \& GRH au sein du Centre d'études et de recherche en gestion d'Aix-Marseille (CERGAM), il est aussi Dean of Faculty de I'IAE et membre de son Conseil d'Institut. II y dirige le Master 2 Management des ressources humaines et de la relation en apprentissage. Ses fonctions à la Commission recherche et au Conseil académique d'Aix-Marseille Université lui ont permis de côtoyer les problématiques des enseignants-chercheurs au niveau local, et comme membre de l'institut Créativité et Innovations d'AixMarseille (InCIAM), il collabore avec des chercheurs hors de sa section CNU. Sur le plan scientifique, ses travaux portent depuis plus de 25 ans sur les questions de justice et de stress dans la GRH, tout particulièrement dans la gestion des carrières. Sur ces thématiques, il est l'auteur de nombreuses publications et communications, sur lesquelles il a dirigé ou codirigé 11 thèses.

Jean-François SATtIN est maître de conférences HDR en sciences de gestion et du management à l'Université Paris 1 Panthéon-Sorbonne, où il codirige le Master 2 Innovation, management des technologies et entrepreneuriat. Membre du laboratoire PRISM-Sorbonne et secrétaire général de l'Académie de l'entrepreneuriat et de l'innovation (AEI), ses travaux portent sur la finance entrepreneuriale ainsi que sur les conséquences des échecs entrepreneuriaux, appréhendées à la fois sous l'angle financier et sous l'angle psychologique. Sur ces thématiques, il est l'auteur de nombreuses contributions dans des revues académiques, dans des ouvrages collectifs, ainsi que dans des congrès nationaux et internationaux. II est vice-président de la section 06 du CNU pour la mandature 2019-2023.

Isabelle SAUVIAT est maître de conférences en sciences de gestion et du management à I'Université de Limoges, en poste à I'IAE de Limoges où elle dirige le Master 2 Management et administration des entreprises. Depuis janvier 2021, elle est vice-présidente déléguée RH et dialogue social à I'Université de Limoges. Précédemment responsable d'un parcours de Master spécialisé en management public pendant douze ans à I'IAE de Tours, ses thèmes de recherche portent, en écho, sur la gestion publique des ressources humaines, d'une part, avec des travaux critiques soulignant ses impacts psychologiques négatifs et, d'autre part, l'étude de modèles alternatifs post-new public management, associés à des approches en termes de sens et de valeurs.

Nathalie SCHIEB-BIENFAIT est maître de conférences HDR en sciences de gestion et du management à l'Université de Nantes, où elle est en poste à I'IAE de Nantes, en charge du Master 2 / Executive MBA management et administration des entreprises. Membre du Laboratoire d'économie et de management de Nantes Atlantique (LEMNA), du Labex Entreprendre (Université de Montpellier) et du bureau de I'Académie de l'entrepreneuriat, où elle coanime les groupes thématiques, elle est rédactrice en chef associée de la Revue de l'Entrepreneuriat. Ses recherches portent sur les dynamiques entrepreneuriales en conjuguant différents niveaux d'analyse (individuel, organisationnel, territorial). Après avoir travaillé sur les démarches de création d'entreprise, les dispositifs d'accompagnement entrepreneurial et la compétence entrepreneuriale, ses travaux portent actuellement sur le travail entrepreneurial et les dispositifs de gestion associés. Ils ont donné lieu à plus de 
140 publications (articles dans revues classées, ouvrages, communications, rapports de recherche).

Éric SÉVERIN est professeur des universités en sciences de gestion et du management à I'Université de Lille, ou il a dirigé la licence de Sciences de Gestion de I'IAE entre 2012 et 2018, et y dirige actuellement le Master recherche en finance, comptabilité et contrôle et le Master 2 comptabilité contrôle audit en apprentissage. Ses recherches, qui portent sur la qualité de l'information, la structure financière, la gouvernance et la défaillance, et plus spécialement la prévision des faillites, ont donné lieu à de nombreuses publications dans des revues académiques et professionnelles. Ancien directeur adjoint du laboratoire Rime Lab jusqu'en 2020, il est responsable de l'axe de recherche GRAF du laboratoire LUMEN depuis 2021. 



\section{Table des matières}

Aude Deville, Jérôme Dupuis, Jean-Fabrice Lebraty, Emmanuelle NèGre,

Caroline RıchÉ et Jean-François SATtIN

Introduction générale

\section{Mesurer}

Aude Deville et Emmanuelle NÈGre

Chiffres et décision

La part des pairs

Éric SÉVERIN et Jérôme DupUIS

Évaluer l'évaluation de la recherche

Le cas des enseignants-chercheurs en sciences de gestion

et du management

Nicolas Aubert, Marc Bonnet, Aude Deville et Anouk Grevin

Vers la reconnaissance de la valeur du don

Un parcours au sein de la section 06 du CNU

Jean-François Sattin et Sophie Cros

L'évaluation de carrière des enseignants-chercheurs

Origines, enjeux et perspectives

\section{Transmettre}

Marc Bollecker, Kirsten Burkhardt et Evelyne Poincelot

L'enseignant-chercheur en sciences de gestion et du management

à l'Université

(Ré)concilier production et diffusion de la connaissance

Nathalie OrIOL et Mickaël DAVID

L'innovation pédagogique à l'université

Dispositifs de mise en œuvre et de valorisation

Élodie Gardet et Caroline Riché

Innovations pédagogiques en sciences de gestion et du management

Le rôle croissant de la gamification et de la ludification

\section{S'interroger}

Hanane BedDi et Olivier Roques

Équité, justice organisationnelle et stress au travail

Le rôle du CNU dans la carrière des enseignants-chercheurs 
Thérèse Albertinı, Delphine Berinı et Solange Hernandez

Et si on parlait de l'attractivité du métier d'enseignant-chercheur en gestion?

Marc Bonnet, Florence Noguera, Jocelyn Husser et Françoise Goter

La recherche à forts impacts sociaux

Enjeux scientifiques et utilité sociétale

Jean-Philippe DeNIS

La valeur de I'indépendance

\section{Explorer}

François Grima et Irène GeORGESCU

Un panorama de la face sombre de la motivation de service public

Effets inattendus sur les attitudes et comportements individuels

François Grima et Olivier MeIER

Gestion des terrains sensibles en sciences de gestion et du management

François Fulconis et Gilles PACHÉ

La logistique

Un champ disciplinaire singulier des sciences de gestion

et du management

Blandine Ageron, Omar Bentahar et Smail Benzidia

Perspectives logistiques pour le système de santé

Enjeux technologiques et durables

\section{Se projeter}

Marine Legall-Ely, Jérémy Morvan, Isabelle Sauviat et Nathalie Schieb-Bienfait

Conseil académique - Conseil national des universités

Deux instances aux missions complémentaires au service de la reconnaissance des enseignants-chercheurs

Marc BidAn, Jean-Fabrice Lebraty, Jean-François Lemoine et Nicolas LesCA

Technologies et CNU

Une nécessaire synergie

Anne Goujon Belghit et Jocelyn Husser

L'héritage universitaire pour les jeunes de demain

Aude Deville

Conclusion générale

Bibliographie générale

Les auteurs 


\section{Travail}

\section{Gouvernance}

(Suite de la page 2)

Pierre Gensse, Éric Séverin et Nadine Tournois, dir., L'entreprise revisitée. Méditations comptables et stratégiques, 216 p., 2015

Nathalie Fabbe-Costes et Gilles Paché, dir., La logistique. Une approche innovante des organisations, 272 p., 2013

Katia Richomme-Huet, Gilles Guieu et Gilles Paché, dir., La démarche stratégique. Entreprendre et croître, 254 p., 2012

Ghislaine Gallenga, dir., De la porosité des secteurs public et privé. Une anthropologie du service public en Méditerranée, 220 p., 2012 
Création graphique de la couverture : Hokus Pokus - Rennes Réalisation de la couverture : Valérie Julia - PUP - Aix-en-Provence Mise en pages : Jean-Bernard Cholbi - PUP - Aix-en-Provence

Imprimé en France

Service imprimerie de l'université d'Aix-Marseille - PSI - Aix-en-Provence

Dépôt légal $1^{\text {er }}$ trimestre 2022

ISBN 979-10-320-0364-0

ISSN 2263-3901 


\section{LA DISPUTATIO}

\section{AU CEUR DU MANAGEMENT DÉBATS ET CONTROVERSES}

TRAVAIL

\section{\& GOUVERNANCE}

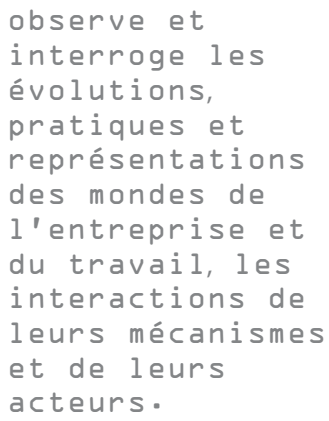

Apparues dans le paysage académique au tournant des années 1970, après s'être émancipées des sciences juridiques et des sciences économiques, les sciences de gestion et du management occupent désormais une place centrale dans le dispositif universitaire français. En effet, un étudiant sur cinq y suit un cursus généraliste ou spécialisé en management des organisations, avec des taux d'insertion professionnelle atteignant des niveaux très élevés. Le dynamisme est au moins aussi significatif en matière de recherche, avec la présence d'associations académiques très impliquées dans la diffusion des savoirs théoriques et appliqués. Alors même que de multiples débats se font jour sur la gouvernance du système universitaire, notamment dans le processus de recrutement et de promotion des enseignants-chercheurs, il est essentiel de discuter sereinement des enjeux en présence. Tel est l'objet du présent ouvrage, dont les différents chapitres sont rédigés par 40 membres ou anciens membres de la section 06 du Conseil national des universités (CNU) de la mandature 2019-2023. Leur volonté est d'éclairer les controverses en optant pour une vision réflexive, fondée sur le registre de la disputatio: une discussion entre pairs mobilisant arguments et contre-arguments. Les 18 chapitres, organisés en cinq parties (mesurer, transmettre, s'interroger, explorer, se projeter), abordent des questionnements majeurs devant retenir l'attention de tous les enseignantschercheurs en sciences de gestion et du management et, plus largement, du grand public qui souhaite mieux appréhender la gouvernance du système universitaire.

Aude Deville, Jérôme Dupuis, Jean-Fabrice Lebraty, Emmanuelle Nègre, Caroline Riché et Jean-François Sattin, coordonnateurs de l'ouvrage, sont respectivement présidente et membres ou anciens membres du bureau de la section 06 du CNU pour la mandature 2019-2023.
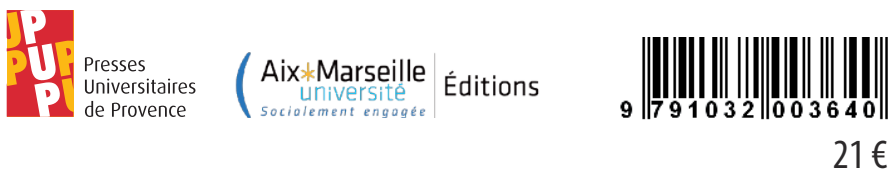